\title{
Two-Photon Frequency Comb Spectroscopy of Atomic Hydrogen
}

\author{
Alexey Grinin
}
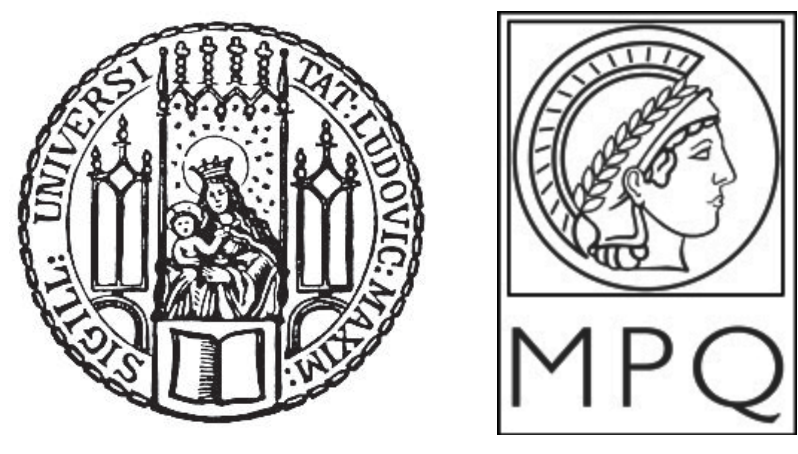

Munich 2020 



\title{
Two-Photon Frequency Comb Spectroscopy of Atomic Hydrogen
}

\author{
Alexey Grinin
}

\author{
Dissertation \\ performed in the Laser Spectroscopy Division \\ of the Max-Planck-Institute for Quantum Optics \\ Garching \\ presented to the Faculty of Physics \\ of the Ludwig-Maximilians-Universität \\ München \\ by \\ Alexey Grinin \\ from Gorbunki
}

Munich, June $30^{\text {th }}, 2020$ 
Erstgutachter: Prof. Dr. Theodor W. Hänsch Zweitgutachter: Prof. Dr. Randolf Pohl

Tag der mündlichen Prüfung: 18. August 2020 


\section{Zusammenfassung}

Quantenelektrodynamik (QED) wird oft als die Krone der modernen Physik bezeichnet, da sie mit erstaunlicher Genauigkeit experimentelle Ergebnisse vorhersagt. Für gegebene Werte der entsprechenden Naturkonstanten können zum Beispiel Energieniveaus im Wasserstoffatom mit Hilfe der QED auf bis zu 13 Stellen genau berechnet werden. Die Entwicklung der QED ging Seite an Seite mit der Präzisionsspektroskopie des einfachsten Atoms im Universum, des Wasserstoffatoms $(\mathrm{H})$. Auch heute bleibt die Wasserstoffspektroskopie unersetzlich für die experimentelle Verifizierung (Falsifizierung) der Quantenelektrodynamik. Um die Energieniveaus im Wasserstoff zu berechnen, benötigt die Theorie je nach Genauigkeit einen oder mehrere Parameter, fundamentale Naturkonstanten, die experimentell bestimmt werden müssen. Die Wasserstoffspektroskopie liefert zwei von ihnen mit höchster Präzision, die Rydbergkonstante $R_{\infty}$ und den Proton-Ladungsradius $r_{p}$ (muonischer Wasserstoff). Zwei weitere Konstanten sind zur Zeit für die Bestimmung der Energieneveaus nötig. Das Elektron zu Proton Massenverhältnis $m_{e} / m_{p}$ und die Feinstrukturkonstante $\alpha$ werden mit Hilfe von Penningtrap- und Atominterferometrieexperimenten bestimmt $[1,2]$. Mit fortschreitender Genauigkeit werden kleinere Effekte berücksichtigt werden müssen und entsprechend weitere Naturkonstanten benötigt (z.B. das Verhältnis der Elektronmasse zur Planck Konstanten $\left.m_{e} / h\right)$.

Die Vermessung der Lambverschiebung im muonischen Wasserstoffatom in 2010 [3], führte zu einem unerwarteten Widerspruch, der auf den Namen Proton Radius Puzzle (PRP) getauft wurde. Im kurzlebigen muonischen Wasserstoff ist das Elektron durch das 200-fach schwerere kurzlebige Muon substituiert. Dadurch ist das Muon etwa 200 mal näher zum Proton und die Abweichung von der punktförmigen Ladungsverteilung des Protons (finite proton size correction) sieben Grössenordnungen grösser als im normalen Wasserstoffatom. Dadurch können diese winzige Energieverschiebung und der Proton-Ladungsradius viel genauer gemessen werden, als das bis dahin mit allen Wasserstoffübergängen möglich war. Allerdings wich der so ermittelte ProtonLadungsradius sieben Standardabweichungen von dem Wert ab, den das Committee on Data for Science and Technology (CODATA) mit Hilfe der Wasserstoffspektroskopiedaten und der Streuungsexperimente an Protonen ermittelt hat [4]. Drei neue Messungen sind inzwischen dazugekommen. Während die Vermessung des 2S-4P Übergangs im Wasserstoffatom [5] und der 2S-2P Übergang [6] mit dem muonischen Wert übereinstimmte, unterstützte das 1S-3S Experiment am LKB in Paris [7] den CODATA 2014 Wert. Die letzte CODATA 2018 [8] Auswertung ist inzwischen online veröffentlicht worden. Der neue Wert des RMS Proton-Ladungsradius stimmt mit den Messungungen des 2S-4P [5] und des 2S-2P Übergänge [6] überein. Allerdings sind keine Details zur Auswertung zur Zeit vorhanden.

Als Ergebnis dieser Arbeit wurde die Unsicherheit des Proton-Ladungsradius und der Rydbergkonstante um einen Faktor von zwei reduziert, verglichen mit der kombinierten Unsicherheit aller im Wasserstoffatom vermessenen Übergänge, inklusive der kürzlich publizierten Erbebnisse am 2S-4P, 2S-2P und 1S-3S Übergängen. Damit ist es die zweitgenaueste Messung im Wasserstoff, die nur der Vermessung des metastabilen 1S-2S Übergangs [9], an Präzision unterliegt. Es ist der erste Übergang im Wasserstoff, der von zwei unabhängigen Gruppen und mit unterschiedlichen Methoden aber mit einer für das Proton Radius Rätsel signifikanten Unsicherheit, gemessen wurde. Die Diskrepanz der Ergebnisse von 2.1 $\sigma$ kombinierten Standardabweichungen deutet darauf hin, dass es sich bei dem Rätsel um ein experimentelles Problem handeln könnte und ermöglicht durch Vergleich und eine wiederholte Analyse der systematischen Fehler, das Problem ausfindig zu machen. Es wurde viel 
experimentelle Arbeit darauf aufgewendet, alle relevanten systematischen Frequenzverschiebungen möglichst klein (kleiner als die PRP Diskrepanz von $7 \mathrm{kHz}$ für $1 \mathrm{~S}-3 \mathrm{~S}$ Übergang) zu halten. Dies wurde vor allem durch einen kryogenen Atomstahl und ein verbessertes Lasersystem möglich. Alle signifikanten systematischen Effekte (inclusive der Druckverschiebung) wurden experimentell und simulationsunabhängig (in erster Ordnung) bestimmt. Die Auflösung der natürlichen Linienbreite von $1 \mathrm{MHz}$ auf einen moderaten Wert von $10^{-3}$ deutet auf weiteres Potenzial dieses Experiments hin. Ferner demonstriert diese Arbeit zum ersten Mal die hochauflösende Frequenzkammspektroskopie im Ultraviolettbereich mit Subkilohertz-Unsicherheit und ist damit wegweisend für die Präzisionsspektroskopie im UV und DUV Bereich, wo nur die Erzeugung von höheren Harmonischen als Laserquelle zur Zeit zur Verfügung stehen.

Unser Ergebnis unterstützt $(1.9 \sigma)$ den Proton-Ladungsradius aus der Spektroskopie am muonischen Wasserstoff und weicht von dem CODATA 2014 Wert um $2.9 \sigma$ kombinierte Standardabweichungen ab. Der Vergleich mit der neuen Messung des 1S-3S Übergangs [7] is limitiert durch die 3.5-fach größere Unsicherheit in [7] und ergibt eine Abweichung von 2.1 $\sigma$ kombinierten Standardabweichungen. Wir bekommen die folgende absolute Frequenz für den $1 \mathrm{~S}-3 \mathrm{~S}(F=1 \mathrm{zu} F=1)$ Übergang im Wasserstoff:

$$
f_{1 \mathrm{~S}-3 \mathrm{~S}}(F=1)=2922742936716.72(72) \mathrm{kHz} .
$$

Nach Abzug der Hyperfeinverschiebung von -341949 069.6(8) Hz [10] ermitteln wir die Zentroidfrequenz des 1S-3S Übergangs zu:

$$
f_{1 \mathrm{~S}-3 \mathrm{~S}}(\text { centroid })=2922743278665.79(72) \mathrm{kHz} \text {. }
$$

Mit Hilfe der 1S-2S Übergangsfrequenz und der Wasserstoffatomtheorie (zusammengefasst in [4]) bekommen wir verbesserte Werte der Rydbergkonstanten und des Proton-Ladungsradius:

$$
\begin{gathered}
R_{\infty}=10973731.568226(38) \mathrm{m}^{-1} \\
r_{p}=0.8482(38) \mathrm{fm} .
\end{gathered}
$$




\section{Abstract}

Quantum electrodynamics (QED) is often considered to be the crown of modern physics in that it is able to predict experiments with astonishing accuracy, reaching, for instance, up to 13 digits of precision for hydrogen energy levels (assuming exact values of required fundamental constants). Due to the simplicity of the hydrogen atom, the development of QED went-side-by side with precision spectroscopy in hydrogen and remains one of the corner stones for testing QED. However the theory depends on four parameters, fundamental constants, which have to be determined experimentally. Precision hydrogen spectroscopy is best at measuring the Rydberg constant $R_{\infty}$, the most precisely known fundamental constant, and the RMS proton charge radius $r_{p}$. The electron to proton mass ratio $m_{e} / m_{p}$ and the fine structure constant $\alpha$ are determined in precision Penning trap and atom interferometry experiments $[1,2]$. At the current level of accuracy the knowledge of these four constants suffices. With higher precision, additional constants, such as the electron mass to Planck constant ratio $\left(m_{e} / h\right)$, are required.

A new intriguing problem, which arose from spectroscopy of muonic hydrogen in 2010, attracted broad interest and is referred to as the Proton Radius Puzzle (PRP). In muonic hydrogen the electron of the hydrogen atom is replaced by the 200 times heavier, short lived muon. As a result of the increased mass the muons orbit is also approx. 200 times closer to the proton. This amplifies the finite proton size correction by almost seven orders of magnitude and allows for very precise determination of $r_{p}$. The measurement of the $2 \mathrm{~S}-2 \mathrm{P}$ transition in muonic hydrogen [3] determined the proton charge radius to be seven combined standard deviations smaller than the value determined in the global adjustment of fundamental constants [4] by the Committee on Data for Science and Technology (CODATA). Three recent measurements in hydrogen with significantly small uncertainties make the problem even more puzzling. While the 2S-4P measurement[5] and the 2S-2P Lamb shift measurement [6] are consistent with the muonic hydrogen value, the $1 \mathrm{~S}-3 \mathrm{~S}$ [7] measurement supports the CODATA 2014 value. The most recent CODATA 2018 evaluation is meanwhile also available online and agrees with the recent measurements of the $2 \mathrm{~S}-4 \mathrm{P}$ [5] and the 2S-2P Lamb shift measurements [6]. However, details of the analysis are not yet available.

One of the important results of this work is a significant improvement of the accuracy of the Rydberg constant and the proton charge radius. The uncertainties on the RMS proton charge radius and the Rydberg constant derived from it are 2 times more precise than the overall previous hydrogen world data including the recent measurements of the $1 \mathrm{~S}-3 \mathrm{~S}, 2 \mathrm{~S}-2 \mathrm{P}$ and $2 \mathrm{~S}-4 \mathrm{P}$ transitions. It is the second most precise measurement in hydrogen after the $1 \mathrm{~S}-2 \mathrm{~S}$ [9], which has orders of magnitude smaller line width than all other transitions. It is the first measurement in hydrogen, which has been performed by two independent groups with different methods and sufficient uncertainty to check consistency within the hydrogen data which is used for proton charge radius determination and therefore sheds light onto possible experimental nature of the discrepancy. All systematic frequency shifts have been reduced to values smaller than the corresponding PRP discrepancy of $7 \mathrm{kHz}$. All significant systematic frequency shifts (including pressure shift) have been measured experimentally and do not rely on simulation to first order. We split the $1 \mathrm{MHz}$ broad $1 \mathrm{~S}-3 \mathrm{~S}$ line by a moderate value of only about $10^{-3}$. Finally, this work demonstrates the first high-resolution spectroscopy below $1 \mathrm{kHz}$ level with a harmonic frequency comb in UV in hydrogen, which is important for future precision spectroscopy experiments in UV and DUV region, where only high harmonic generation as a laser source is 
currently available.

Our result supports $(1.9 \sigma)$ the RMS proton charge radius derived from muonic hydrogen spectroscopy and disagrees with the CODATA 2014 value by 2.9 combined standard deviations. The comparison with the newly obtained value for the $1 \mathrm{~S}-3 \mathrm{~S}$ transition [7] is limited by the 3.5-fold larger uncertainty in [7] and yields a $2.1 \sigma$ combined standard deviations. We obtain the following absolute frequency of the $1 \mathrm{~S}-3 \mathrm{~S}(F=1$ to $F=1)$ transition:

$$
f_{1 \mathrm{~S}-3 \mathrm{~S}}(F=1)=2922742936716.72(72) \mathrm{kHz}
$$

Subtracting the hyperfine shifts of -341949069.6(8) Hz [10] gives the hyperfine centroid:

$$
f_{1 \mathrm{~S}-3 \mathrm{~S}}(\text { centroid })=2922743278665.79(72) \mathrm{kHz} .
$$

In combination with the $1 \mathrm{~S}-2 \mathrm{~S}$ transition frequency and the theory for the energy levels summarized in [4], we obtain an improved value for the Rydberg constant

$$
R_{\infty}=10973731.568226(38) \mathrm{m}^{-1}
$$

and the proton charge radius

$$
r_{p}=0.8482(38) \mathrm{fm} .
$$




\section{Contents}

List of Figures iii

1. Precision Hydrogen Spectroscopy 1

1.1. The Historic Interplay between Hydrogen Spectroscopy and QED . . 1

1.2. QED Description of the Energy Levels in Hydrogen . . . . . . . . . 5

1.3. Proton Radius Puzzle . . . . . . . . . . . . . . . . . . 6

1.4. Advances of the Garching 1S-3S Setup . . . . . . . . . . . . . . . . 9

2. Two-photon Direct Frequency Comb Spectroscopy 11

2.1. Frequency Combs in Spectroscopy . . . . . . . . . . . . . . . . . . . 11

2.2. Basics of Frequency Combs . . . . . . . . . . . . . . . . . . . . . 12

2.3. Continuous Wave Two-Photon Spectroscopy . . . . . . . . . . . . . . 14

2.4. Two-Photon Frequency Comb Spectroscopy . . . . . . . . . . . . . 22

2.5. Monte Carlo simulations . . . . . . . . . . . . . . . . . . . . 27

3. Experimental setup 31

3.1. Frequency measurement . . . . . . . . . . . . . . . . . 31

3.2. Laser system . . . . . . . . . . . . . . . . . . . . . . 34

3.3. Mode suppression . . . . . . . . . . . . . . . . . . . . . . 40

3.4. Vacuum system and enhancement cavity . . . . . . . . . . . . . . . 42

3.5. Atomic hydrogen beam . . . . . . . . . . . . . . . 46

3.6. Fluorescence detection . . . . . . . . . . . . . . . . . . 49

4. Data Evaluation $\mathbf{5 5}$

4.1. Data Description . . . . . . . . . . . . . . . . . 55

4.2. Line fitting and normalization . . . . . . . . . . . . . . 56

4.3. Line width . . . . . . . . . . . . . . . . . . 59

4.4. Global fitting procedure . . . . . . . . . . . . . . . . 61

5. Systematic Uncertainties Analysis $\quad \mathbf{6 5}$

5.1. Chirp induced residual first-order Doppler shift . . . . . . . . . . . 65

5.2. AC-Stark shift . . . . . . . . . . . . . . . . . . 74

5.3. Second-order Doppler shift . . . . . . . . . . . . . . . . 77

5.4. Pressure shift . . . . . . . . . . . . . . . . . . . 81

5.5. DC-Stark shift . . . . . . . . . . . . . . . . 84

5.6. Zeeman shift . . . . . . . . . . . . . . . . . . . . . 88

5.7. Line distorting effects . . . . . . . . . . . . . . . . . 90 
5.8. Quantum interference . . . . . . . . . . . . . . 92

5.9. Incoherent line pulling . . . . . . . . . . . . . . . . . . . . 94

5.9.1. Other line components . . . . . . . . . . . . . . 94

5.9.2. Forbidden $\Delta F=1$ components . . . . . . . . . . . 96

5.9.3. Cavity modulation side bands . . . . . . . . . . . . . . . . . . 99

5.10. Tilted wave fronts . . . . . . . . . . . . . . . . 101

5.11. Cross-talks between different systematic effects . . . . . . . . . . . 104

5.12. Results and Error Budget . . . . . . . . . . . . . . 109

6. Conclusions and Outlook 111

6.1. Discussion of Measurement Results . . . . . . . . . . . . . . . . 111

6.2. Frequency Comb Spectroscopy Technique Investigation Results . . . . 113

6.3. Suggestions for Future Improvements . . . . . . . . . . . . . . . . . 115

$\begin{array}{ll}\text { Appendix } & 117\end{array}$

$\begin{array}{lr}\text { A. Enhancement Cavity Characterization } & 117\end{array}$

A.1. Transmission Measurement . . . . . . . . . . . . . . . . . . 117

A.2. Cavity Waist Measurement and Mode Matching . . . . . . . . . . . . 118

$\begin{array}{ll}\text { B. AC Stark Shift Derivation } & 121\end{array}$

B.1. Fourth Order AC Stark Shift . . . . . . . . . . . . . . . . . . 122

$\begin{array}{lr}\text { Bibliography } & 129\end{array}$

$\begin{array}{lr}\text { Acknowledgments } & 139\end{array}$

$\begin{array}{ll}\text { List of publications and presentations } & 141\end{array}$

$\begin{array}{ll}\text { Declaration of Originality } & 145\end{array}$ 


\section{List of Figures}

1.1. Hydrogen energy diagram showing levels with principal quantum number $n \leq 3 \ldots \ldots \ldots \ldots \ldots \ldots$

1.2. Proton charge radius . . . . . . . . . . . . . . . . . 7

2.1. Frequency comb spectrum . . . . . . . . . . . . . . . . 12

2.2. Two-photon excitation . . . . . . . . . . . . . . . . 15

2.3. Two-photon excitation types . . . . . . . . . . . . . . . . 17

2.4. Doppler free to Doppler broadened contrast comparison. . . . . . . . 22

2.5. Principle of the two-photon frequency comb spectroscopy. . . . . . . . 24

2.6. Steady state solution for DFCS . . . . . . . . . . . . . . . . . 24

3.1. Experimental set-up . . . . . . . . . . . . . . . . . . 32

3.2. Full frequency scan with all fine- and hyperfine $1 \mathrm{~s}-3 \mathrm{~S}$ and $1 \mathrm{~S}-3 \mathrm{D}$ transitions . . . . . . . . . . . . . . . 34

3.3. Comb spectra . . . . . . . . . . . . . . . 36

3.4. Self-phase modulation simulation . . . . . . . . . . . . . . . . . 38

3.5. Vacuum system . . . . . . . . . . . . . . . . . . . . . . . . . . . . . . . . . . . . . 42

3.6. Differential pumping system . . . . . . . . . . . . . . . . . . . 44

3.7. Hydrogen dissociation system . . . . . . . . . . . . . . . . . . 47

3.8. Temperature dependence of the count rates . . . . . . . . . . . . . . . 48

3.9. Drawing of the PCV and detection scheme . . . . . . . . . . . . 50

3.10. Detection profile . . . . . . . . . . . . . . 51

4.1. Histogram of valid data points . . . . . . . . . . . . . 56

4.2. Normalization example scan . . . . . . . . . . . . . . . . 57

4.3. $\chi^{2}$ distribution analysis . . . . . . . . . . . . . . . . . 58

4.4. Correlation between DB and DF count rates . . . . . . . . . . . 59

4.5. Average Line and residuals . . . . . . . . . . . . . . . . . . 60

4.6. Line widths analysis . . . . . . . . . . . . . . . . . . . . . 61

4.7. Global fit $\chi^{2}$-distribution analysis . . . . . . . . . . . . 63

5.1. CIFODS principle . . . . . . . . . . . . . 66

5.2. CIFODS Monte Carlo simulation . . . . . . . . . . . . 68

5.3. Measurement of the CIFODS as a function of position . . . . . . . . . 69

5.4. Linearity of the CIFODS effect . . . . . . . . . . . . . 70

5.5. CIFODS interpolation . . . . . . . . . . . . . . 71

5.6. Experimental chirp distribution . . . . . . . . . . . . . 72 
5.7. AC-Stark shift extrapolation . . . . . . . . . . . . . . . 75

5.8. SOD crossover velocity . . . . . . . . . . . . . . . 78

5.9. SOD simulation . . . . . . . . . . . . . . . . . . 79

5.10. SOD extrapolation . . . . . . . . . . . . . . . . 80

5.11. Amplitudes ratio vs. temperature . . . . . . . . . . . . 82

5.12. Pressure shift extrapolation . . . . . . . . . . . . . . . . 83

5.13. Charging up of the nozzle . . . . . . . . . . . . . . 85

5.14. DC-Stark shift screening and compensation . . . . . . . . . . 86

5.15. DC-Stark shift measurement . . . . . . . . . . . . . . . . . 87

5.16. SOD line shape distortion . . . . . . . . . . . . . . . . . 91

5.17. SOD line shape distortion frequency shift . . . . . . . . . . . . . 92

5.18. Quantum interference . . . . . . . . . . . . . . . . 93

5.19. Tilted wave fronts Doppler shift . . . . . . . . . . . . . 101

5.20. Tilted wave fronts Doppler shift extrapolation . . . . . . . . . . 104

5.21. SOD mimics CIFODS . . . . . . . . . . . . . . . 106

A.1. A scheme of the UV enhancement cavity of the $1 \mathrm{~S}-3 \mathrm{~S}$ experiment together with a beam profiler/power meter . . . . . . . . . . . 118

A.2. Determination of the cavity waist using a beam profiler . . . . . . . 119

A.3. Enhancement cavity transmitted power cavity modes . . . . . . . . 120

A.4. White light interferogram of the cavity mirrors . . . . . . . . . . . . . 121 


\section{Precision Hydrogen Spectroscopy}

\subsection{The Historic Interplay between Hydrogen Spectroscopy and QED}

The hydrogen atom has played without doubt a key role in the development of atomic physics and quantum mechanics [11]. Being a two-body system it was possible to calculate its spectrum analytically and thus over a time span of 60 years a series of refinements of theory and experiment has been performed, finally resulting in the formulation of the quantum electrodynamics (QED), which is often stated as the best theory in physics. It is capable of predicting the energy levels of atomic hydrogen and the electron $g$ factor with an incredible accuracy and served as the blueprint for other quantum field theories.

The first detailed spectrum of hydrogen has been published by Anders Jonas Ångström in 1862 [12]. Johann Jakob Balmer in 1885 provided an empirical formula for the wavelengths of the Balmer series $(n=3,4,5, \ldots \rightarrow n=2,[13])$, observed in the spectrum of hydrogen and Johannes Robert Rydberg generalized it to include all wavelengths in 1888. In it's modern version the Rydberg formula for the hydrogen energy levels is:

$$
E_{n}=-\frac{R_{\infty}}{n^{2}}
$$

Where $E_{n}$ is the energy of the level with the principal quantum number $n$ and $R_{\infty}$ the Rydberg constant.

The advances in the understanding of the structure of atoms through scattering experiments by Ernest Rutherford in 1911 built the ground for the development of the first quantum theory by Niels Bohr in 1913. Bohr's atomic model assumes a heavy nucleus consisting of a positively charged proton and a light negatively charged electron orbiting around the proton bound by the Coulomb force. The angular momentum of the electron is assumed to take only integer numbers of the Planck constant $\hbar$ and can be interpreted as a standing matter wave with the de Broglie wavelength.

The interpretation of the electron as a standing matter wave lead to a search for a matter wave equation, which was formulated by Erwin Schrödinger in 1926.

$$
i \hbar \frac{\partial}{\partial t} \Psi(\vec{r}, t)=\left[\frac{-\hbar^{2}}{2 m} \nabla^{2}+V(\vec{r}, t)\right] \Psi(\vec{r}, t)
$$

Here $\Psi(\vec{r}, t)$ is the complex wave function of the electron (or any other quantum particle), the square modulus of which is interpreted (Max Born) as the probabi- 
lity distribution of the electron to be at a certain place $\vec{r}$ at the time $t$. Just as trajectories were now replaced by probability distributions, physical quantities like the kinetic energy and potential were replaced by operators $\frac{-\hbar^{2}}{2 m} \nabla^{2}, V(\vec{r}, r)$ (for instance Coulomb potential). Schrödinger's equation 1.2 remains the working horse of quantum mechanical calculations till today and is by no means restricted to the simple hydrogen atom. The Rydberg formula 1.1 could be derived formally with it and also the line intensities were explained for the first time. The solutions of the Schrödinger equation have besides the principal quantum number $n$ two angular momentum quantum numbers $l=0,1, \ldots, n$ and $m_{l}=-l,-l+1, \ldots, l-1, l$. The energies of the levels with the same principal quantum number $n$ however are predicted by the Schrödinger equation to have the same energy, i.e. to be degenerate.

Already in 1887, long before Bohr and Schrödinger proposed their theories, Michelson and Morley [14] showed by means of Fourier spectroscopy that the Balmer- $\alpha$ line $(n=3 \rightarrow n=2)$ is a doublet. This so called fine splitting is a relativistic correction due to electrons motion and its spin, which lifts the degeneracy of the levels with the same principal quantum number $n$ but different total angular momentum $(J=L+S)$ quantum number $j$ (capital letters denote operators, corresponding small letters their eigenvalues). The electron's spin, an intrinsic angular momentum of the electron, which has no classical equivalent was first observed in the famous SternGerlach experiment in 1921, predating the Schrödinger theory and was postulated by Uhlenbeck and Goudsmit in 1925. It was added ad hoc to the Schrödinger equation. Using the relativistic energy momentum relation other relativistic corrections could also be derived for special cases (Arnold Sommerfeld). While the spin-orbit correction separately depends on all quantum numbers $(n, l, s)$ and the relativistic velocity change of the electron mass depends on $n$ and $l$, only the total angular momentum quantum number $j$ remains in their sum. The fine splitting scales with the square of the fine-structure constant $\alpha \approx 1 / 137$ and with the fourth power of the charge number $Z$ (for hydrogen-like ions with larger nuclear charge).

$$
\Delta E_{n, l, j}=\alpha^{2}\left(\frac{3}{4 n}-\frac{1}{j-1 / 2}\right) \frac{Z^{4}}{n^{3}} 2 \pi h c R_{\infty}
$$

Here $\Delta E_{n, l, j}$ is the energy difference between fine structure components $(j=l+1 / 2$ and $j=l-1 / 2), Z$ is the nucleus charge.

A similar though several orders of magnitude smaller effect to the spin-orbit coupling is the hyperfine structure, which is due to the coupling between the nuclear spin $I$ and the angular momentum $L$ and spin $S$ of the electron (total angular momentum $F=L+S+I$ ). First measurements were already performed by Michelson in 1881 but could be only explained when Wolfgang Pauli proposed the nuclear spin in 1924.

These important results could first rigorously be derived by Paul Dirac in 1928 . The Dirac equation was the first fully relativistic matter wave equation, satisfying inherently the Lorentz invariance and relativistic energy momentum relation. The 
theory was very successful and Dirac was able to predict the existence of spin and anti matter (e.g. positrons). However the degeneracy of levels with the same total angular momentum $j$ in eq. 1.3 was found to be the next stumbling block.

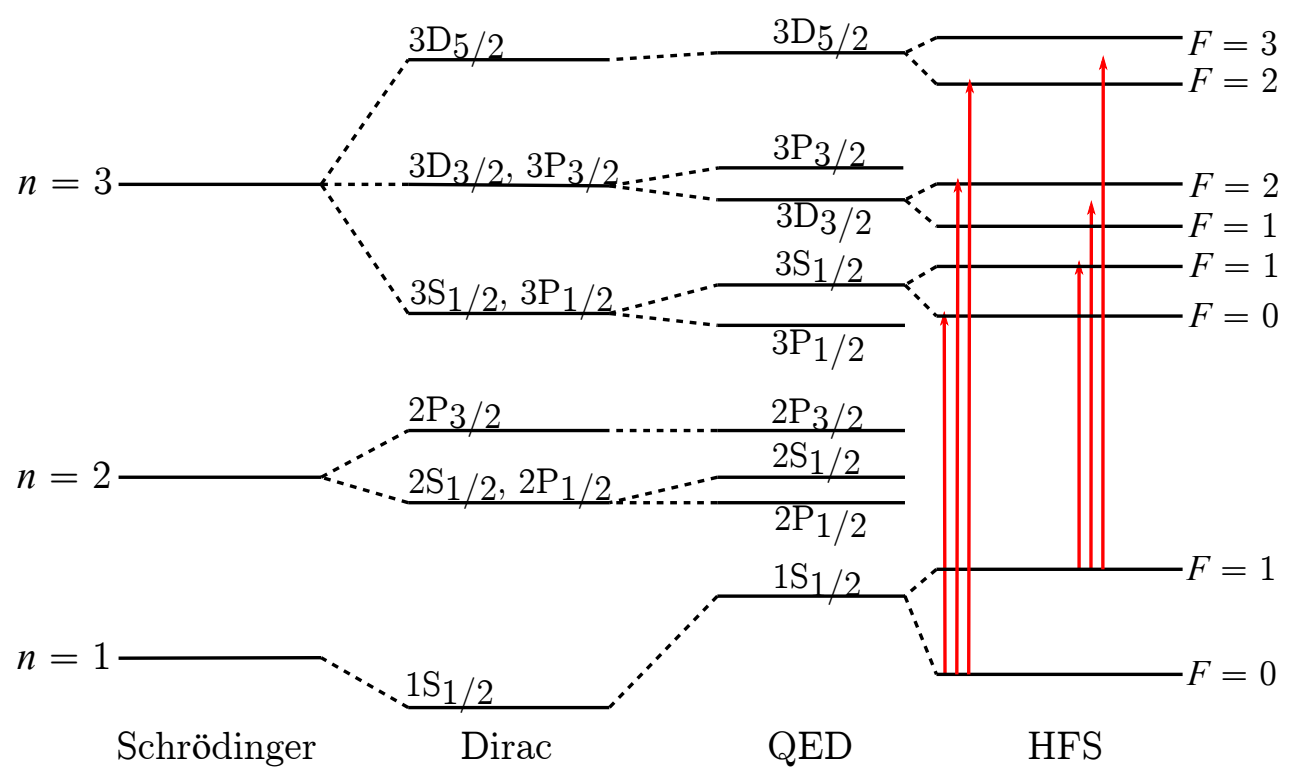

Figure 1.1.: Hydrogen energy diagram showing levels with principal quantum number $n \leq 3$. The energy differences are not true to scale. From left to right the development from Bohr/Schrödinger, Dirac to QED is shown. The hyperfine structure is shown only for the relevant states for two-photon excitation from the ground state $n=1$ to $n=3$. Allowed two-photon transitions for the 1S-3S experiment, which is the subject of this work, are displayed with red arrows (compare fig. 3.2). The degenerate $m_{j}$ magnetic sublevels of the total angular momentum are not shown. Spectroscopic notation is used, where S, $\mathrm{P}, \mathrm{D}$ correspond to $l=0,1,2$, total angular momentum excluding nuclear spin is given by the subscript and $F$ is the total angular momentum including nuclear spin.

In 1947 Willis Lamb and Robert Retherford observed that the $2 \mathrm{~S}_{1 / 2}$ state is shifted upward with respect to the $2 \mathrm{P}_{1 / 2}$ state by approximately $1 \mathrm{GHz}$, by directly driving the $2 \mathrm{~S}_{1 / 2}-2 \mathrm{P}_{1 / 2}$ transition with microwave radiation. This shift was named the Lamb shift. The advantage of using microwave instead of optical excitation is the small absolute value of the first order Doppler shift due to the small transition frequency as compared to optical frequencies $\left(\Delta \nu_{D}=\nu_{0} v / c\right)$. This epochal experiment not only demonstrated the superiority of stimulated resonances in atomic beams as compared to spontaneous decay in discharge tubes but also triggered a new round of theory refinements.

Soon after the discovery of the Lamb shift modern quantum electrodynamics was developed by pioneering works of S. Tomonaga, J. Schwinger, R. Feynman and others and it was shown that the tiny shift is caused by vacuum quantum fluctuations. The vacuum is quantized and being in its ground state virtual photon and electron pairs are created and annihilated, perturbing the electron. This effects are sensitive to 
different electronic wave functions due to their different spatial distributions. QED has survived since than for over 70 years and more than six orders of magnitude improvement of experimental precision. Until today precision hydrogen spectroscopy remains a key tool for testing QED and determining the fundamental constants such as the Rydberg constant or the root-mean square proton charge radius. New exciting problems such as the proton radius puzzle, which will be discussed in the next section, make possible that new fundamental discrepancies could be found and shed light on the most fundamental laws of nature.

Figure 1.1 summarizes the level structure within the different models, spanning about 60 years of the development of QED. The energy differences are not true to scale. From left to right the historic development from Bohr/Schrödinger, Dirac to QED is shown. The hyperfine structure levels are displayed only for the relevant states for two-photon excitation from the ground state $n=1$ to $n=3$. Allowed two-photon transitions for the 1S-3S experiment, which is the subject of this work, are displayed with red arrows. The degenerate $m_{j}$ magnetic sub-levels of the total angular momentum are not shown. Spectroscopic notation is employed, where S, P, $\mathrm{D}$ correspond to $l=0,1,2$, total angular momentum excluding nuclear spin is given by the subscript and $F$ is the total angular momentum including nuclear spin. 


\subsection{QED Description of the Energy Levels in Hydrogen}

While the simple Bohr model only needs the Rydberg constant $R_{\infty}$ to convert atomic units into SI units for the energy equation 1.1, including other effects such as relativistic effects, recoil effects, QED vacuum fluctuations effects, finite proton charge distribution etc., obviously complicate the energy relation and require knowledge of additional constants, such as the fine- structure constant $\alpha$, the electron to proton mass ratio $m_{e} / m_{p}$ and the root-mean square (RMS) proton charge radius $r_{p}$. At a level of accuracy, which is not reached currently by experiment, also other constants such as the ratio of the electrons mass to Planck's constant $m_{e} / h$ would enter. The full description of the terms can be found in [4]. We restrict our self to the general formula, showing only the main Bohr/Schrödinger contribution, the QED series $f_{n, \ell, j}\left(\alpha, \frac{m_{e}}{m_{p}}, \ldots\right)$ in the fine structure constant $\alpha$ and separately the finite proton size contribution. The resulting simplified formula for the QED energy levels of atomic hydrogen reads:

$$
E_{n, \ell, j}=R_{\infty}\left(-\frac{1}{n^{2}}+f_{n, \ell, j}\left(\alpha, \frac{m_{e}}{m_{p}}, \ldots\right)+\delta_{\ell, 0} \frac{C_{\mathrm{NS}}}{n^{3}} r_{p}^{2}\right)
$$

with $n, \ell$ and $j$ being the principle quantum number, and the orbital and total angular momentum, respectively.

In principle, to fit $N$ unknown parameters from the relation 1.4 (fundamental constants), one only needs to measure $N$ transitions. By observing the residuals between the model 1.4 and the measurement using the best fit parameters, one can already judge about the correctness of the model. With any additional independent measurement the model would be stronger restricted and possible statistically significant discrepancies would need to be attributed to either experimental or calculation errors or to limitations of the theory itself. In principle the quantum electrodynamics could be falsified this way. It turns out that hydrogen spectroscopy is best in determining only two of the four relevant constants, namely, the Rydberg constant $R_{\infty}$ and the RMS proton charge radius $r_{p}$. Two other constants are determined from other experiments, where they not merely enter as small contributions, but in leading order effects. The fine structure constant is determined from precision measurements of the electron $g$-factor [1] and the electron to proton mass ration $m_{e} / m_{p}$ is determined from Penning trap experiments [2]. It is therefore of fundamental scientific interest to measure more transitions and repeat measurements improving uncertainties. 


\subsection{Proton Radius Puzzle}

We consider the finite proton charge radius correction in eq. 1.4. The proton (nucleus) is not a point-like particle, but rather its charge has a spherically symmetric distribution with the root mean squared radius $r_{p}$. Thus, the electron sees a reduced potential and the energy levels are therefore slightly different than for the Coulomb potential (for the simple model of a homogeneously charged sphere, the potential is different from the Coulomb potential only inside the charged sphere). Here, we are interested to show the scaling of this energy correction with the mass of the particle (electron, muon) and the principal quantum number $n$. An illustrative calculation using the simple model of a homogeneously charged sphere can be found in [15]. The difference potential $W(r)$ between the coulomb potential of a point-like particle and the potential of the charge distribution can be considered as perturbation and its effect on the energy levels can be calculated in first order perturbation theory. The energy shifts in first order perturbation theory are simply the expectation value of the perturbation potential in the corresponding eigenstates. Since the potential is spherically symmetric the angular part of the wave function $Y_{l}^{m *}(\phi, \theta) Y_{l}^{m}(\phi, \theta)$ just integrates to one and we are left with:

$$
\Delta E_{n, l, m}=\left\langle\psi_{n, l, m}|W| \psi_{n, l, m}\right\rangle=\int_{0}^{\infty} R_{n, l}^{*}(r) R_{n, l}(r) W(r) \mathrm{d} r
$$

Where $R_{n, l}(r)$ is the radial part of the hydrogen eigenstate $\left|\psi_{n, l, m}\right\rangle$. The characteristic extent of the electrons wave function is the Bohr radius $a_{0}=\epsilon_{0} h^{2} / \pi m_{e} e^{2} \approx 0.5 \AA$, which is five orders of magnitude larger than the RMS proton charge radius $r_{p} \approx 1 \mathrm{fm}$. Thus the radial part of the wave function in eq. 1.5 can be approximated by its value at the origin $R_{n, l}(0)$, which is nonzero only for s-states $(l=0)$. Thus we obtain:

$$
\Delta E_{n, l, m}=\left|R_{n, l}(0)\right|^{2} \delta_{l 0} \int_{0}^{\infty} W(r) \mathrm{d} r \propto 1 / n^{3} a_{0}^{3}
$$

In other words the finite proton charge radius correction is proportional to the probability density of the electron (muon) to be at the origin. For s-states the probability density at the origin is inversely proportional to the cube of the principal quantum number and the Bohr radius $\left|R_{n, l}(0)\right|^{2} \propto 1 / n^{3} a_{0}^{3}$. The mass of the muon and thus its Bohr radius $a_{0}=\epsilon_{0} h^{2} / \pi m_{\mu} e^{2}$ is 200-times smaller than for the electron. Therefore the finite proton charge radius correction in muonic hydrogen is $200^{3} \approx 10^{7}$ seven orders of magnitude larger than in ordinary hydrogen (also the absolute frequency for a given transition is $\approx 200$ times larger). A new intriguing problem arose from precision spectroscopy of the muonic hydrogen in 2010 and attracted broad interest. It was coined the the Proton Radius Puzzle (PRP). The measurement of the Lamb shift (2S-2P transition) in muonic hydrogen [3] determined the proton charge radius to be 4 combined standard deviations smaller than the value determined from the regular atomic hydrogen. Taking into account the scattering data the discrepancy is determined in the global adjustment of fundamental constants [4] by the Committee 


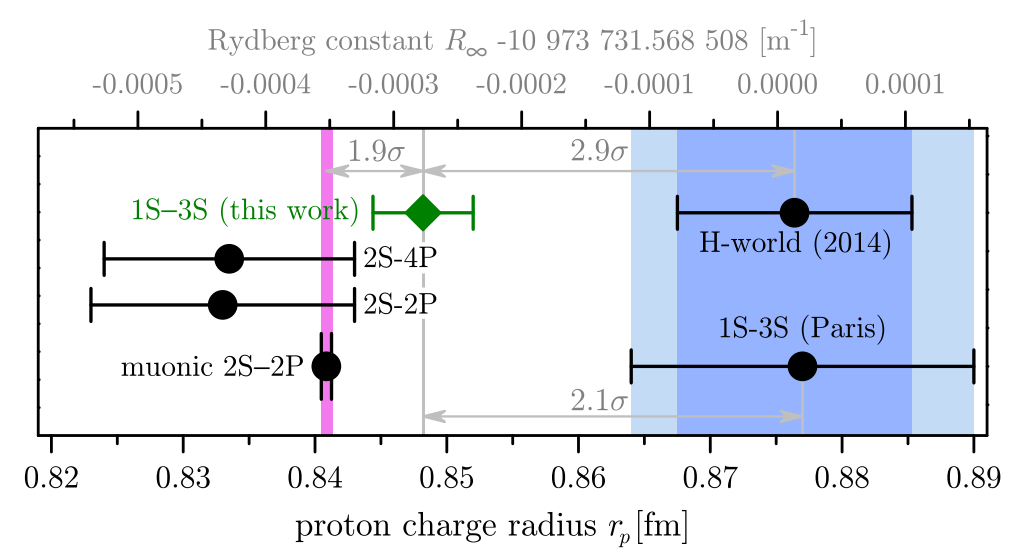

Figure 1.2.: The proton charge radius measurements (bottom axis) as obtained from various experiments partially disagree preventing a verification of quantum electrodynamics at the level of experimental uncertainties. Combining the 1S-2S and the 1S-3S ("this work") transition frequencies in atomic hydrogen gives a significantly smaller value than the previous world data ("H-world (2014)") obtained by using all hydrogen measurements (15 lines) available for the most recent published CODATA 2014 adjustment (Adj \#8 table XXIX in [4]). In contrast, a recent continuous wave measurement of the 1S-3S transition frequency ("Paris") [7], confirms the CODATA value. Our result is in reasonable agreement with a value derived from the previous $2 \mathrm{~S}-4 \mathrm{P}$ measurement [5], with a radio frequency measurement of the $2 \mathrm{~S}-2 \mathrm{P}$ transition [6] as well as with the value obtained from muonic hydrogen [16]. Due to the strong correlation (98.91\%) between $R_{\infty}$ and $r_{p}$, one can display the data also in terms of the Rydberg constant in the same graph (upper axis).

on Data for Science and Technology (CODATA) to be 7 combined standard deviations. The electron-proton scattering data however leads to mixed results, which either lead to an increase of the "proton radius puzzle" [4] or a decrease [17].

Several new precision measurements have been performed recently. Precise measurements of the $2 \mathrm{~S}-4 \mathrm{P}(2017)$ and the $2 \mathrm{~S}-2 \mathrm{P}$ transition frequencies $[5,6](2019)$ are in agreement with the muonic hydrogen value, while a previous measurement of the 1S-3S transition is not [7] (2018).

Here I report a measurement that significantly reduces the uncertainty of the 1S-3S transition frequency making it the second most precisely known transition frequency in atomic hydrogen. It is topped only by the $1 \mathrm{~S}-2 \mathrm{~S}$ transition [9] that has several orders of magnitude smaller line width than any other relevant transition in atomic hydrogen. We have significantly reduced systematic uncertainties and required corrections that are now about an order of magnitude smaller than the "proton radius puzzle". The remaining systematic effects have been determined experimentally and do not rely on model assumptions. In addition, we do not use complicated line shape models and find the line center within $10^{-3}$ of the line width, which is a rather moderate value.

With this experiment we also demonstrate the first high-resolution laser spectroscopy with a harmonic frequency comb in the ultraviolet region. The associated 
short pulses help to avoid the photo-refractive effect in non-linear crystals that has long hindered precision laser spectroscopy of the 1S-3S transition [18]. In the future, it may allow one to push the wavelengths to even shorter unexplored wavelength regions using high harmonic generation that hopefully will enable high resolution laser spectroscopy of hydrogen-like ions for the first time [19].

Combining the results for the $1 \mathrm{~S}-3 \mathrm{~S}$ and the $1 \mathrm{~S}-2 \mathrm{~S}$ transitions, we extract values for the Rydberg constant $R_{\infty}$ and the RMS proton charge radius $r_{p}$. These new values are two times more accurate than the ones obtained from all previous hydrogen data combined. By using only two measurements to determine two constants/parameters, nothing can be said about the validity of QED. To check for consistency one needs additional measurements. It does not matter whether we use the Rydberg constant or the RMS proton charge radius for this consistency check because the values of these parameters are strongly correlated through (1.4). In the current situation this analysis yields mixed results. While this work favours the data from muonic hydrogen and recently improved measurement of the $2 \mathrm{~S}-2 \mathrm{P}$ Lamb shift in regular hydrogen [6], it deviates by $2.1 \sigma$ from a recently published measurement of the $1 \mathrm{~S}-$ $3 \mathrm{~S}$ transition frequency obtained by $\mathrm{H}$. Fleurbaey and co-workers with a continuous laser [7]. Further the RMS proton charge radius and the Rydberg constant, derived from this measurement, are in very good agreement with the values derived from the recent measurement of the $2 \mathrm{~S}-4 \mathrm{P}$ interval but disagrees by $2.9 \sigma$ with the hydrogen world data values evaluated by CODATA. Figure 1.2 summarises this situation. 


\subsection{Advances of the Garching $15-3 S$ Setup}

The 1S-3S transition in hydrogen is very attractive for determination of the Rydberg constant and the RMS proton charge radius and tests of QED for several reasons. First it is a two-photon transition and thus the first order Doppler shift is strongly suppressed (see sections 5.1, 5.10). Second, it has a relatively small natural line width of only $\gamma=1.005 \mathrm{MHz}$. Besides the $1 \mathrm{~S}-2 \mathrm{~S}$ transition with several orders of magnitude smaller line width, only two-photon transitions to higher S and D states (2S-8S/8D for instance) possess even smaller natural line widths. However, those transitions are much more sensitive to DC Stark shifts due to the large spatial extent of the electronic wave function, which poses a serious experimental challenge. Quantum interference (compare section 5.8) is typically also a more important issue for these transitions because of the dense level structure. On the contrary the DC Stark shift coefficient of the $1 \mathrm{~S}-3 \mathrm{~S}$ transition is only about $7 \mathrm{~Hz}(\mathrm{~V} / \mathrm{m})^{-2}$ (see section 5.5). Further, the low principal quantum numbers make the finite proton size correction relatively large.

The first precision measurement of the 1S-3S transition was performed in 2010 by $\mathrm{O}$. Arnoult in the group of F. Biraben at the Laboratoire Kastler Brossel (LKB) in Paris [20] with an uncertainty of $13 \mathrm{kHz}$. In 2016 our group at the Max-Planck institute of Quantum Optics performed a measurement with an uncertainty of $17 \mathrm{kHz}$ [21]. In 2018 H. Fleurbaey from the LKB group performed an improved measurement [7] and combined with the previous measurement [20] obtained an uncertainty of $2.6 \mathrm{kHz}$.

We should stress, that the 1S-3S transition is the only transition in hydrogen, which has been measured by two independent groups with sufficient low uncertainty to contribute to the PSP and the constant determination. The groups use two different techniques (continous wave vs. frequency comb, room temperature vs. cryogenic hydrogen) and thus have different leading systematic frequency shifts. The measurement presented in this work has an uncertainty of $0.72 \mathrm{kHz}$ and differs in several aspects from previuos measurements. First it is the first $1 \mathrm{~S}-3 \mathrm{~S}$ transition measurement performed with cryogenically cooled hydrogen atoms $(T=7 \mathrm{~K})$, which reduces the second order Doppler shift (SOD, see section 5.3) from roughly $120 \mathrm{kHz}$ to $3 \mathrm{kHz}$. Second we could improve our laser system and the detection efficiency to achieve a statistical uncertainty of $70 \mathrm{~Hz}$ only, which is more than one order of magnitude better than any previous result. With such a high signal strength we could afford a direct measurement of all main systematic frequency shifts based on linear interpolation and extrapolation in corresponding quantities (AC Stark shift in power see section 5.2, CIFODS in chirp see section 5.1, pressure shift in atomic density, see section 5.4 and the SOD in temperature, see section 5.3). This is a very reliable method since it does not depend on simulations since the nonlinearities in our case can be shown to be negligible. We believe that the present result can be further improved. 



\section{Two-photon Direct Frequency Comb Spectroscopy}

\subsection{Frequency Combs in Spectroscopy}

Frequency combs have revolutionized the field of spectroscopy [22] and found numerous applications in other fields [23], [24]. For its invention T.W. Hänsch and J.L. Hall were rewarded with a Nobel prize in physics in 2005 together with R.J. Glauber for his contributions to the theory of quantum optics. The broad spectrum of a frequency comb together with the regularly spaced mode structure serve as an optical "ruler", with which the high optical frequency of a spectroscopy laser can be measured very precisely. Prior to frequency combs long phase locked frequency chains covering the whole range from radio frequency standards to the optical domain needed to be built and operated.

Long before the ground breaking application of frequency combs for absolute frequency determination they have been suggested for direct use in precision spectroscopy experiments as the excitation source. E.V. Baklanov and V.P. Chebotaev suggested in 1976 [25] to use frequency combs to drive the 1S-2S two-photon transition in hydrogen. Contrary to one-photon transitions, where only one comb mode can be resonant with the transition, in a two-photon transitions all pairs of modes whose frequencies add up to the transition frequency can contribute. The resulting excitation rate is the same as for an excitation with a continuous wave (CW) laser with the same average power. Further the line width of the transition is not broadened by the large spectral width of the comb but rather is determined by the narrow line width of the comb modes. Also the AC Stark shift is given by the average intensity of the pulses rather than by the peak intensity [26]. This remarkable features of the Direct Frequency Comb Spectroscopy have been soon after proposal demonstrated by the group of T.W. Hänsch at Stanford in 1977 [27, 28] in a sodium $3 \mathrm{~S}-5 \mathrm{~S}$ and $3 \mathrm{~S}-4 \mathrm{~S}$ transitions. The advantage of using frequency combs instead of $\mathrm{CW}$ lasers is the high efficiency of pulsed lasers for nonlinear frequency conversion in crystals and gases. This opens the doors of precision spectroscopy in DUV and XUV regions, where no CW laser is available even today. 


\subsection{Basics of Frequency Combs}

As the name suggests an optical frequency comb is a regularly spaced array of laser frequencies

$$
\omega_{n}=n \omega_{r}+\omega_{C E}
$$

Where $\omega_{r}=1 / 2 \pi T$ is the repetition rate, $n$ is the mode number starting from the so called carrier envelope frequency $\omega_{C E}$. In the time domain it corresponds to an infinite repetitive train of equal pulses. Figure 2.1 shows an example of the time and frequency domain pictures for such a frequency comb. As is well known from the

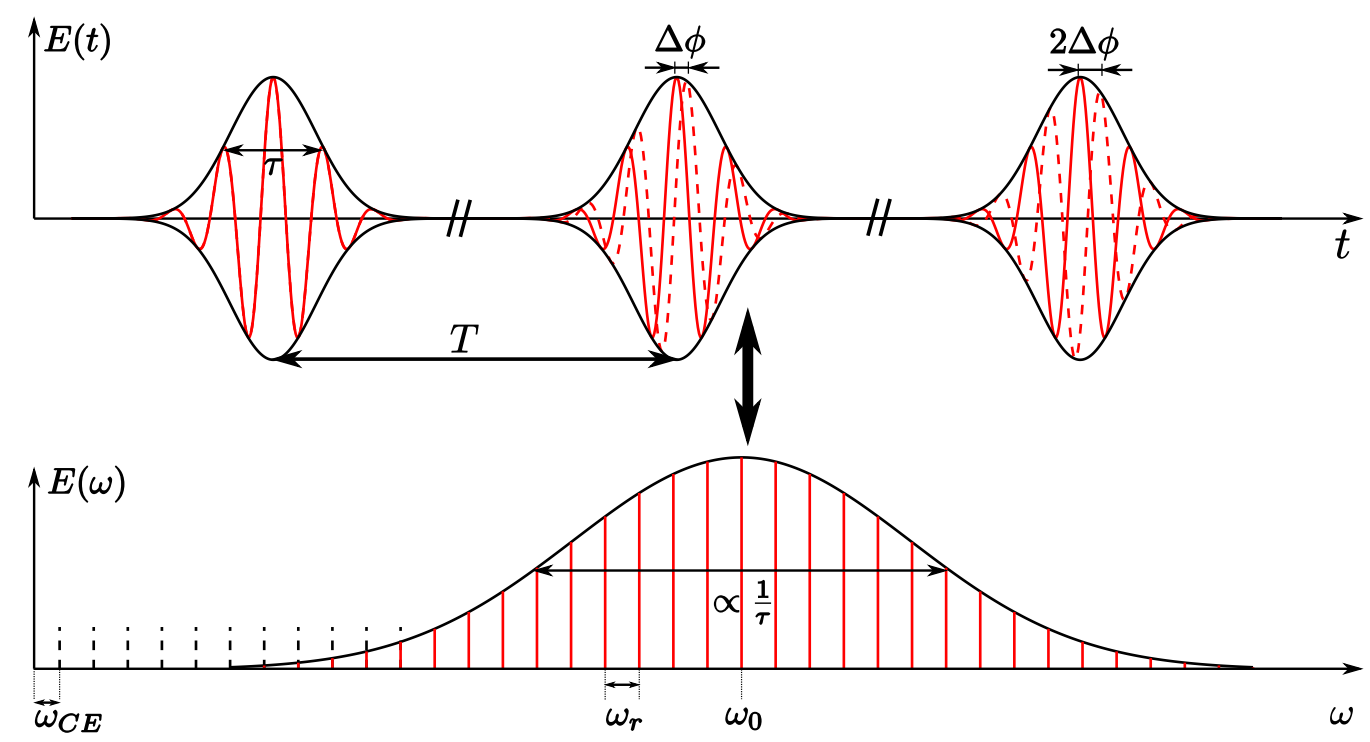

Figure 2.1.: Time and frequency domain pictures of a frequency comb. In the time domain a frequency comb is a repetitive pulse train with the repetition rate $\omega_{r}$. The actual shape of the pulse is irrelevant as long it it the same for all pulses. For this example Gaussian pulses with FWHM pulse duration $\tau$ are drawn. The carrier envelope phase $\phi_{C E}$ can change from one pulse to the next by $\Delta \phi$, leading to a frequency shift of all comb modes by the carrier envelope frequency $\omega_{C E}$. In frequency domain such a pulse train corresponds to a regularly spaced comb of $\delta$-functions. The envelope corresponds to the Fourier transform of the time domain envelope.

Fourier decomposition any repetitive structure in time domain can be decomposed into a series of sines with the repetition frequency $\omega_{r}$ and its overtones $n \omega_{r}$. On the other hand we could see it as a pure sinusoidal wave (red curve) with the frequency $\omega_{0}$, referred to as the carrier frequency, which is amplitude modulated by the envelope (black curve). The modulation leads to side bands at multiples of the modulation frequency $\omega_{r}$. The carrier frequency can be associated with the central mode of the comb. The carrier envelope phase $\phi_{C E}$, which is the phase between the peak of the envelope and the underlying oscillating field can change from pulse to pulse by $\Delta \phi$ as in figure 2.1. A phase difference in the time domain translates into a frequency 
shift of all combs by the carrier envelope frequency $\omega_{C E}=\Delta \phi / T$. Experimentally a frequency comb can be realized by a mode-locked laser. In the laser resonator the pulse envelope propagates with the group velocity $v_{g}$ and the carrier with the phase velocity $v_{p}$ which are usually not equal due to the dispersion of the intra cavity mirrors, crystals or any other optical elements. Thus the pulse-to-pulse phase shift between the envelope and the carrier is:

$$
\Delta \phi=\omega_{0}\left(\frac{L}{v_{g}\left(\omega_{0}\right)}-\frac{L}{v_{p}}\right)
$$

Generally the dispersion can be characterized by the frequency dependent wave number $k(\omega)$, which can be decomposed into Tailor series around the central mode $\omega_{0}$. The round trip phase $\phi_{n}$ in the resonator of the mode $\omega_{n}$ reads:

$$
2 L\left[k\left(\omega_{0}\right)+k^{\prime}\left(\omega_{0}\right)\left(\omega_{n}-\omega_{0}\right)+\frac{k^{\prime \prime}\left(\omega_{0}\right)}{2}\left(\omega_{n}-\omega_{0}\right)^{2}+h . o .\right]=2 \pi n+\Delta \phi_{n}
$$

where $L$ is the length of the resonator. The phase difference between two neighboring modes is given by:

$$
k^{\prime}\left(\omega_{0}\right) \omega_{r}+\frac{k^{\prime \prime}\left(\omega_{0}\right)}{2}\left[\left(\omega_{n+1}-\omega_{0}\right)^{2}-\left(\omega_{n}-\omega_{0}\right)^{2}\right]+\text { h.o. }=\frac{2 \pi+\Delta \phi_{n+1}-\Delta \phi_{n}}{2 L}
$$

The first order derivative of the wave number with respect to $\omega\left(k^{\prime}\left(\omega_{0}\right)=v_{g}^{-1}\right)$ is simply the inverse of the group velocity. The second order derivative $k^{\prime \prime}(\omega)$ is the group velocity dispersion (GVD) (linear chirping), which makes the pulse envelope spread while it propagates. For a constant mode spacing the repetition rate $\omega_{r}$ needs to be independent on the mode number $n$. In eq. 2.4 this means that all terms, except of the first term $k^{\prime}\left(\omega_{0}\right) \omega_{r}$ need to be zero for all mode numbers $n$, which is only possible if all derivatives of $k^{(p)}\left(\omega_{0}\right)$ vanish. Further the mode to mode phase shift $\Delta \phi_{n+1}-\Delta \phi_{n}$ can not depend on $n$ and thus must be a constant. In this way the modes are "locked". This remarkable property of mode-locked lasers can be verified by simply observing the spectrum of the pulses over time. A tiny deviation of $1 \mathrm{~Hz}$ would destroy the pulse after already $1 \mathrm{~s}$. An observation of a constant pulse shape even after hours of operation demonstrates the vanishing of these terms. The width of the comb modes is in reality not a $\delta$-function even for an infinitely long pulse train. Phase noise and amplitude noise lead to a broadening of the comb modes. A detailed description of the frequency comb theory can be found in [29], written by one of the inventors of the frequency comb and my supervisor Thomas Udem. 


\subsection{Continuous Wave Two-Photon Spectroscopy}

In this section the basic theory of two-photon spectroscopy for one-photon forbidden dipole transitions is introduced for single frequency (CW) excitation. Based on these general results, two-photon direct frequency comb spectroscopy is discussed in the next section and differences between CW and frequency comb excitations are highlighted.

For states with the same parity one-photon dipole transitions are forbidden and the corresponding dipole moment matrix elements vanish, (e.g. for $1 \mathrm{~S}-3 \mathrm{~S}$ transition)

$$
\hat{\vec{d}}_{e, g}=\langle e|q \vec{r}| g\rangle \stackrel{\text { same parity }}{=} 0
$$

We use the terms initial state and ground state $|g\rangle$ as synonyms, although for a particular transition, the state $|g\rangle$ might not correspond to the actual ground state of the atom. One-photon forbidden transitions can be two-photon allowed over some intermediate state. In this case the first photon excites the atom from the ground state $|g\rangle$ to some intermediate off-resonant state $|n\rangle$ and the second photon excites the atom to the final state $|e\rangle$. An intermediate state can be any state of the atom, to which one-photon dipole transitions from the initial and to the final state are allowed. If an intermediate state is off-resonant with respect to the laser frequency, it is very short lived, and thus is referred to as a virtual state. The populations of the intermediate states can be therefore neglected and an effective two-level atom model can be applied. For 1S-3S transition in hydrogen, which is the subject of this work, this is extremely well justified, since $205 \mathrm{~nm}$ laser light is nanometers off from even the nearest one-photon $1 \mathrm{~S}-2 \mathrm{P}$ transition. In case, where any intermediate state is near resonant with any of the frequencies of the excitation source, the dynamics is very different and a three or more level model needs to be worked with. For the $1 \mathrm{~S}-3 \mathrm{~S}$ and $1 \mathrm{~S}-3 \mathrm{D}$ transitions in hydrogen the intermediate states are all $n \mathrm{P}$-states of the discrete spectrum of hydrogen and $\mathrm{P}$-states of the continuous spectrum. Figure 2.2 shows a simplified two-photon transition energy diagram. It should be noted, that virtual states $|n\rangle$ do not necessarily need to be in-between of the ground and excited states.

We seek to derive the effective second order interaction Hamiltonian, which couples two states of the same parity $|g\rangle$ and $|e\rangle$ over intermediate states $|n\rangle$.

$$
\hat{H}=\hat{H}_{0}+\hat{H}_{1}
$$

Where $\hat{H}_{0}$ is the free Hamiltonian of the system and $\hat{H}_{1}$ is the desired effective twophoton Hamiltonian. $\hat{H}_{1}$ can be written as a sum of four matrix elements of the effective two-level atom.

$$
\hat{H}_{1}=H_{1, g g}|g\rangle\left\langle g\left|+H_{1, e e}\right| e\right\rangle\left\langle e\left|+H_{1, g e}\right| e\right\rangle\left\langle g\left|+H_{1, e g}\right| g\right\rangle\langle e|
$$

The off-diagonal elements $H_{1, e g}$ couple the two states $|g\rangle$ and $|e\rangle$, while the diagonal elements constitute the so called second order AC Stark shift. We assume, 


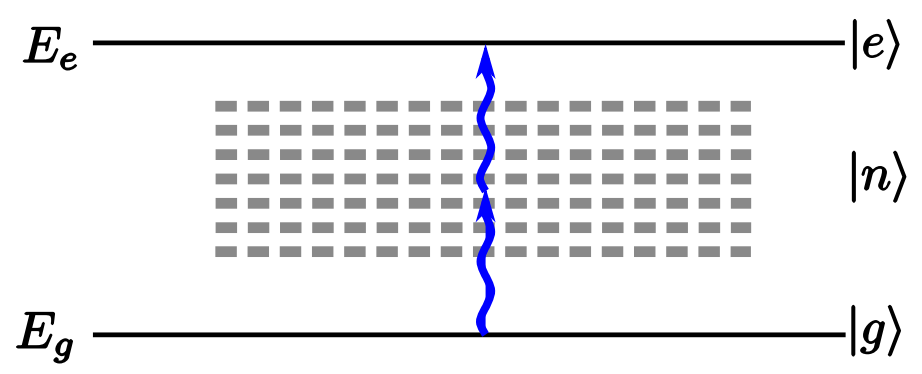

Figure 2.2.: Two-photon excitation of a transition between two states $|g\rangle$, $|e\rangle$ with the same parity and energies $E_{g}$ and $E_{e}$ over one-photon allowed off-resonant intermediate states $|n\rangle$.

that the interaction is mediated by the dipole operator $\hat{V}(\epsilon, t)=e E_{L} z e^{-\epsilon|t|} \cos (\omega t)$, which is a monochromatic plane wave, polarized in the $z$-direction and adiabatically damped at the distant past and future. The electric field is assumed to be constant within the atom (dipole approximation), which is a good approximation as long as the wavelength $\lambda$ is large compared to the typical size of the atom (Bohr radius). Analysis of the deviation from the dipole approximation can be found in [30]. The solution can be written as a superposition of the ground and excited states $|\psi(t)\rangle=e^{-i \omega_{g} t} c_{g}(t)|g\rangle+e^{-i \omega_{e} t} c_{e}(t)|e\rangle$. To calculate $|\psi(t)\rangle$ in second order perturbation theory, we transform the state $\left|\psi_{I}(t)\right\rangle=e^{-\frac{i}{\hbar} \hat{H}_{0} t}|\psi\rangle=c_{g}(t)|g\rangle+c_{e}(t)|e\rangle$ into the interaction picture and make use of the time evolution operator $\left|\psi_{I}(t)\right\rangle=$ $\hat{U}_{I}(\epsilon, t)\left|\psi_{I}(-\infty)\right\rangle=\hat{U}_{I}(\epsilon, t)|g\rangle$. The perturbation series for the time evolution operator is given by the Dyson series.

$$
\hat{U}_{I}(\epsilon, t)=1-\frac{i}{\hbar} \int_{-\infty}^{t} d t^{\prime} \hat{V}_{I}\left(\epsilon, t^{\prime}\right)+\left(-\frac{i}{\hbar}\right)^{2} \int_{-\infty}^{t} d t^{\prime} \int_{-\infty}^{t^{\prime}} d t^{\prime \prime} \hat{V}_{I}\left(\epsilon, t^{\prime}\right) \hat{V}_{I}\left(\epsilon, t^{\prime \prime}\right)+\ldots
$$

Where $\hat{V}_{I}(\epsilon, t)=e^{\frac{i}{\hbar} \hat{H}_{0} t} \hat{V}(\epsilon, t) e^{-\frac{i}{\hbar} \hat{H}_{0} t}$ is the dipole potential in the interaction picture. When computing the matrix elements, the first order term vanishes, since both states $|g\rangle$ and $|e\rangle$ are parity eigenstates with the same eigenvalue and $\hat{V}(\epsilon, t) \propto r$. We calculate the projection $\left\langle\phi \mid \psi_{I}(t)\right\rangle$ of the state $\left|\psi_{I}(t)\right\rangle$ to some state $|\phi\rangle$, where $|\phi\rangle$ is either the ground state or the excited state.

$$
M=\left\langle\phi\left|\hat{U}_{I}(\epsilon, t)\right| g\right\rangle=\left(-\frac{i}{\hbar}\right)^{2} \int_{-\infty}^{t} d t^{\prime} \int_{-\infty}^{t^{\prime}} d t^{\prime \prime}\left\langle\phi\left|\hat{V}_{I}\left(\epsilon, t^{\prime}\right) \hat{V}_{I}\left(\epsilon, t^{\prime \prime}\right)\right| g\right\rangle
$$

We insert an identity operator and write the dipole interaction potential explicitly 
as a sum of exponentials.

$$
\begin{aligned}
M= & \left.\left(-\frac{i e E_{L}}{2 \hbar}\right)^{2} \sum_{n, \pm-\infty} \int_{-\infty}^{t} d t^{\prime} \int_{-\infty}^{t^{\prime}} d t^{\prime \prime} e^{ \pm i \omega_{1} t^{\prime}-\epsilon\left|t^{\prime}\right|} e^{ \pm i \omega_{2} t^{\prime \prime}-\epsilon \mid t^{\prime \prime}}\right|_{\times} \\
& \left\langle\phi\left|e^{\frac{i}{\hbar} H_{0} t^{\prime}} \hat{z} e^{-\frac{i}{\hbar} H_{0} t^{\prime}}\right| n\right\rangle\left\langle n\left|e^{\frac{i}{\hbar} H_{0} t^{\prime \prime}} \hat{z} e^{-\frac{i}{\hbar} H_{0} t^{\prime \prime}}\right| g\right\rangle \\
= & \left(-\frac{i e E_{L}}{2 \hbar}\right)^{2} \sum_{n, \pm-\infty} \int_{-\infty}^{t} d t^{\prime} \int_{-\infty}^{t^{\prime}} d t^{\prime \prime} e^{ \pm i \omega_{1} t^{\prime}+i \omega_{\phi n} t^{\prime}-\epsilon\left|t^{\prime}\right|} e^{ \pm i \omega_{2} t^{\prime \prime}+i \omega_{n g} t^{\prime \prime}-\epsilon \mid t^{\prime \prime}} \mid\langle\phi|\hat{z}| n\rangle\langle n|\hat{z}| g\rangle
\end{aligned}
$$

The \pm -sum runs over all possible signs combinations of $\omega_{1}$ and $\omega_{2}$ in this equation $\left(\omega_{1}+\omega_{2},-\omega_{1}+\omega_{2}, \omega_{1}-\omega_{2},-\omega_{1}-\omega_{2}\right)$, which we have denoted by introducing two different frequencies $\omega_{1,2}$ to better keep track of the signs. Next, we evaluate the integrals, where $e^{-\epsilon|t|}$ assures the convergence. We further take the limit $\epsilon \rightarrow 0$, assuming long enough interaction time.

$$
M(t)=\left(-\frac{i e E_{L}}{2 \hbar}\right)^{2} \sum_{n, \pm} \frac{\langle\phi|\hat{z}| n\rangle\langle n|\hat{z}| g\rangle e^{i\left(\omega_{\phi g} \pm \omega_{1} \pm \omega_{2}\right) t}}{\left[i\left(\omega_{n g} \pm \omega_{2}\right)\right]\left[i\left(\omega_{\phi g} \pm \omega_{1} \pm \omega_{2}\right)\right]}
$$

We are interested in the excitation probability $P_{\phi g}$ from the ground state $|g\rangle$ to the final state $|\phi\rangle$ in the time interval of $t=-T / 2 \ldots T / 2$, which can be obtained using the Schrödinger equation $\hat{H}_{1, I}\left|\psi_{I}(t)\right\rangle=i \hbar \partial_{t}|\psi(t)\rangle$

$$
\begin{aligned}
P_{\phi g} & =\left|\int_{-T / 2}^{T / 2}\left\langle\phi\left|\hat{H}_{1}\right| \psi_{I}(t)\right\rangle d t\right|^{2}=\left|\int_{-T / 2}^{T / 2} i \hbar\left\langle\phi\left|\frac{\partial}{\partial t}\right| \psi_{I}\right\rangle d t\right|^{2}=\left|\int_{-T / 2}^{T / 2} i \hbar \frac{\partial}{\partial t} M(t) d t\right|^{2} \\
& =|\frac{\left(e E_{L}\right)^{2}}{4 \hbar} \underbrace{}_{n, \pm} \frac{\langle\phi|\hat{z}| n\rangle\langle n|\hat{z}| g\rangle}{\omega_{n g} \pm \omega_{2}}|^{2} \underbrace{\left(\int_{-T / 2}^{T / 2} e^{-i\left(\omega_{\phi g} \pm \omega_{1} \pm \omega_{2}\right) t} d t\right)}_{\rightarrow \delta\left(\omega_{\phi g} \pm \omega_{1} \pm \omega_{2}\right), \text { for } T \rightarrow \infty}\left(\int_{-T / 2}^{T / 2} e^{i\left(\omega_{\phi g} \pm \omega_{1} \pm \omega_{2}\right) t} d t\right)
\end{aligned}
$$

As expected, the excitation probability grows linearly and the excitation probability per unit time is a constant.

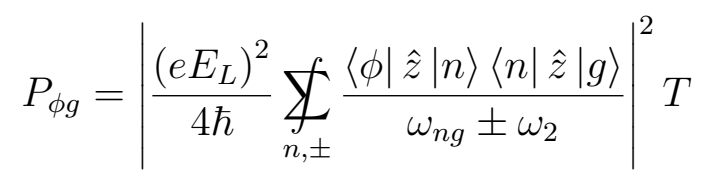

There are four possible types of two-photon interactions (excitation from $|g\rangle$ to $|e\rangle$, deexcitation from $|e\rangle$ to $|g\rangle$, AC Stark shift coupling between $|g\rangle$ and $|n\rangle$ and AC Stark shift coupling between $|e\rangle$ and $|n\rangle$ ), as is illustrated in figure 2.3. We first 


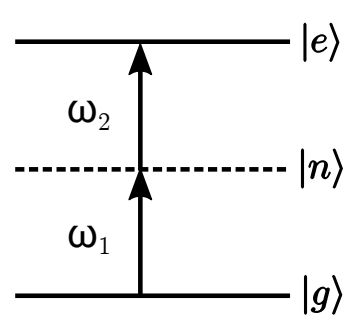

Excitation

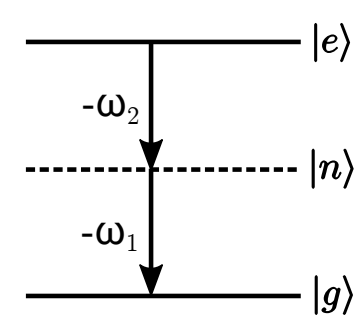

Deexcitation

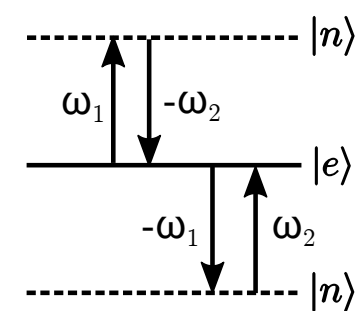

AC Stark shift

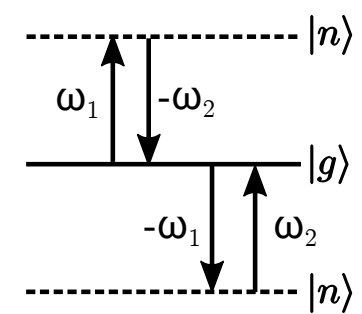

AC Stark shift

Figure 2.3.: The four possible two-photon interaction types are shown, corresponding to (de)exctitation and second order AC Stark shift of the ground and excited states, as given by the different sign combinations in eq. 2.13

consider the probability for the atom to be excited from the ground state $|g\rangle$ to the excited state $|\phi\rangle=|e\rangle$. The energy conservation in this case demands $\omega_{e g}=$ $\omega_{1}+\omega_{2}=2 \omega$. One can easily show, using the left part of the Schrödinger equation $\hat{H}_{1, I}\left|\psi_{I}(t)\right\rangle=i \hbar \partial_{t}|\psi(t)\rangle$, that the time-dependent off-diagonal matrix element of the two-photon dipole interaction Hamiltonian $\hat{H}_{1, e g}$, which satisfies equation 2.12 , is given by the following expression.

$$
\hat{H}_{1, e g}=\frac{e^{2}}{\hbar} \sum_{n} \frac{\langle e|\hat{z}| n\rangle\langle n|\hat{z}| \phi\rangle}{\omega_{n g}-\omega} E(t)^{2}=2\left(2 \pi c \epsilon_{0}\right) \beta_{e g} E(t)^{2}
$$

Note, that since (de)excitation requires the frequencies of the two photons to have the same sign, the total two-photon matrix element is proportional to the square of the field $E(t)^{2}$ and not the intensity $I(t) \propto E^{*}(t) E(t)$, as opposed to the AC Stark shift, which is discussed below. We have defined the time-independent two-photon matrix element $\beta_{\text {eg }}[30]$.

$$
\beta_{e g}=\frac{e^{2}}{2 h c \epsilon_{0}} y_{n} \frac{\langle e|\hat{z}| n\rangle\langle n|\hat{z}| \phi\rangle}{\omega_{n g}-\omega}
$$

As explained above, the sum (integral for continuum states) in 2.15 runs over all eigenstates $|n\rangle$ of the free Hamiltonian of the system $\hat{H}_{0}$ with eigenenergies $E_{n}$ and the contribution of each intermediate level is given by its one-photon dipole elements with the ground and excited states weighted by the laser frequency detuning from the intermediate transition (cf. fig. 2.2). $\beta_{\text {eg }}$ can be calculated using explicit expressions for the matrix elements of the discrete and continuum spectrum of hydrogen [31] and the eigenstates of the gross structure (quantum numbers $n, l$ ). Alternatively, one can use the explicit expressions for both discrete and continuum spectrum of the Sturmian expansion [30]. Generally, for an arbitrary polarization, the two-photon transition operator can be written as following [30]

$$
T^{i j}=r^{i} \frac{1}{H_{0}-E_{g}-\hbar \omega} r^{j}
$$




\section{Two-photon Direct Frequency Comb Spectroscopy}

Where $r^{i}$ represents any Cartesian coordinate. The eigenstates of the real hydrogen atom include fine- and hyperfine levels. Fortunately for S-S transitions, the twophoton operator has isotropic symmetry such that it transforms under rotation as a scalar. Thus $\beta_{g e}$ for fine-structure and hyperfine structure can be obtained using the Wigner-Eckart theorem, where $n$ is the principal quantum number, $S$ electron spin, $L$ angular momentum, $I$ nuclear spin, $\hat{J}=\hat{L}+\hat{S}$ and $\hat{F}=\hat{L}+\hat{S}+\hat{I}$ total angular momentum [30].

$$
\begin{aligned}
\beta_{g e}= & -\frac{e^{2}}{4 \pi \hbar^{2} c \epsilon_{0}}(-1)^{F^{\prime}-m_{F}^{\prime}}\left(\begin{array}{ccc}
F^{\prime} & 2 & F \\
-m_{f}^{\prime} & 0 & m_{F}
\end{array}\right) \sqrt{(2 F+1)\left(2 F^{\prime}+1\right)} \times \\
& (-1)^{J^{\prime}+I+F+2}\left\{\begin{array}{ccc}
J^{\prime} & F^{\prime} & I \\
F & J & 2
\end{array}\right\} \sqrt{(2 J+1)\left(2 J^{\prime}+1\right)} \times \\
& (-1)^{L^{\prime}+S+J+2}\left\{\begin{array}{ccc}
L^{\prime} & J^{\prime} & S \\
J & L & 2
\end{array}\right\} \times\left\langle n^{\prime} L^{\prime}|| \boldsymbol{T}(2)|| n L\right\rangle
\end{aligned}
$$

With this equations $\beta_{\text {eg }}$ can be calculated for any hyper-fine level and any polarization of the electric field. The $3 \mathrm{j}$ and $6 \mathrm{j}$ symbols are defined in [32]. For some transitions and certain polarizations $\beta_{g e}$ is zero and thus the transition is forbidden. The resulting selection rules for two-photon dipole transitions are discussed in [33], [34].

Next, we consider the AC Stark shift case, in which the atom is initially in the ground or excited state $|\phi\rangle=|g\rangle,|e\rangle$ (S-state) then excited to any of the intermediate P-states $|n\rangle$ and then back to the $|\phi\rangle$ (see fig. 2.2). Thus the energy conservation demands $\omega_{1}=-\omega_{2}$ and we obtain from the equation 2.13.

$$
\Delta E_{A C}^{\phi} \equiv \hat{H}_{1, \phi \phi}=\frac{e^{2}}{\hbar} \sum_{n, \pm} \frac{\langle\phi|\hat{z}| n\rangle\langle n|\hat{z}| \phi\rangle}{\omega_{n g} \pm \omega} \underbrace{E^{*}(t) E(t)}_{\frac{2 I(t)}{c \epsilon_{0}}}
$$

There is an important difference to the two-photon diagonal element in eq. 2.14. As explained above, the two photons have frequencies with opposite signs (first excited then deexcited or vice versa), such that the total matrix element is proportional to the intensity of the field rather than the square. Summing up the AC Stark shift of the ground and excited states, the total transition frequency is shifted by:

$$
\delta \nu_{A C}=\frac{1}{h}\left(\Delta E_{A C}^{e}(t)-\Delta E_{A C}^{g}(t)\right)=\left(\beta_{A C}(e)-\beta_{A C}(g)\right) I(t)
$$

Where we $\beta_{A C}(\phi)$ is defined analogue to $\beta_{e g}$ as following and is calculated and tabulated for S-S-transition in hydrogen in [30].

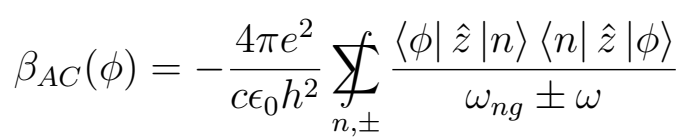


The second order AC Stark shift is proportional to the intensity $\propto I$. The fourth order of the AC Stark shift, which is proportional to the square of the intensity $\propto I^{2}$, is calculated in section B.

So far only one field has been assumed. The presented results can be immediately extended to the case of two-counter propagating fields with angular frequencies $\omega_{1,2}$ and wave numbers $k_{1,2}$, which are polarized along the $z$-axis.

$$
E(t, z)=E_{1}(t, z)+E_{2}(t, z)=\frac{1}{2}\left[E_{01} e^{-i\left(\omega_{1} t+k_{1} z\right)}+E_{02} e^{-i\left(\omega_{2} t-k_{2} z\right)}\right]+\text { c.c. }
$$

The total Hamiltonian $\hat{H}$ is given by the following expression.

$$
\hat{H}=\left[\hbar \omega_{g}+\Delta E_{A C}^{g}(t)\right]|g\rangle\left\langle g\left|+\left[\hbar \omega_{e}+\Delta E_{A C}^{e}(t)\right]\right| e\right\rangle\langle e|+\frac{\hbar \Omega(t)}{2}(|e\rangle\langle g|+| g\rangle\langle e|)
$$

This is the same Hamiltonian as the two-level atom one-photon dipole interaction, except that the one-photon Rabi frequency is replaced by the two-photon Rabi frequency $\Omega(t)$.

$$
\Omega(t)=2\left(2 \pi c \epsilon_{0} \beta_{g e}\right) E(t, z)^{2}=2\left(2 \pi c \epsilon_{0} \beta_{g e}\right)(\underbrace{E_{1}(t, z)^{2}+E_{2}(t, z)^{2}}_{\text {Doppler broadened }}+\underbrace{2 E_{1}(t, z) E_{2}(t, z)}_{\text {Doppler free }})
$$

If the two beams are coming from the same laser and are counter propagating $\left(k_{1}=\right.$ $-k_{2}$ ), then the product $E_{1} E_{2}$ is Doppler free. However different deviations can occur. For instance the wave vectors $\vec{k}_{1}, \vec{k}_{2}$ might be not perfectly anti parallel within the entire excitation volume, such that some residual Doppler shift occurs. We refer to it as "tilted wave front Doppler shift" (see. section 5.10). Note, that in the case of a linearly chirped frequency comb, the product of the fields has a position dependent parabolic phase along the pulse collision volume, which leads to an important comb spectroscopy specific systematic residual first order Doppler-shift (see section 5.1).

Finally, just as in the one-photon case, we can derive the optical Bloch equations, describing the population dynamics of the two levels. To this end we use the Hamiltonian 2.22 and the Von Neumann equation for the density operator $\hat{\rho}$.

$$
i \hbar \frac{d \hat{\rho}}{d t}=[\hat{H}(t), \hat{\rho}]+i \hbar \Gamma(\rho)
$$

Where $\Gamma(\rho)$ is the Lindblad operator, which describes the decay terms. The resulting set of differential equations is given in section 2.5, eq. 2.43, where the Monte Carlo simulation procedure is explained, which has been realized by Arthur Matveev [35] and used throughout the whole work on this experiment, to understand and verify the different aspects of the experiment.

It is instructive to calculate the steady-state solutions for a CW laser from the optical Bloch equations 2.43. Since the equations are linear the frequency comb case 


\section{Two-photon Direct Frequency Comb Spectroscopy}

can be constructed from it as a sum of resonant pairs of comb modes. To this end we set the derivatives in eq. 2.43 to zero and obtain the following solution.

$$
R_{C W}^{D F}=\Gamma \rho_{e e}=\frac{\Gamma \Omega^{2} / 4}{\left(\omega_{e g}-2 \omega\right)^{2}+\Gamma^{2} / 4+\Omega^{2} / 2} \approx \frac{\Gamma \Omega^{2} / 4}{\left(\omega_{e g}-2 \omega\right)^{2}+\Gamma^{2} / 4}
$$

In the last step $\Omega^{2} / 2$ can be neglected in the denominator, if the Rabi frequency $\Omega=2\left(2 \pi \epsilon_{0} c\right) \beta_{g e} E_{0}^{2}$ is much smaller than the natural line width $\Gamma$ (for $1 \mathrm{~S}-3 \mathrm{~S}, 80 \mu \mathrm{m}$, $0.1 \mathrm{~W}$ per direction, $\Omega=625 \mathrm{~Hz} \ll \Gamma=2 \pi \times 1.005 \mathrm{MHz}$ ). Care must be taken when deriving the steady state solution as it is, strictly speaking, only valid for very slow atoms, which propagate only a small distance within the life time of the excited state $1 /(2 \pi \Gamma)$. In this case the transverse and longitudinal position dependence of the fields can be neglected (but not the standing wave). As expected the steady state solution is a Lorentzian line. $R_{C W}^{D F}=\Gamma \rho_{e e}$ is the Doppler free (DF) count rate, i.e. the number of photons per unit of time per atom emitted when the atom decays to the ground state. As expected the count rate is proportional to the intensity squared $\Omega^{2} \propto I^{2}$, which is due to the two-photon nature of the transition. The detuning $\Delta \omega=2 \omega-\omega_{e g}$ is the difference between the resonance frequency $\omega_{e g}$ and twice the laser frequency $\omega$. For a laser power of $0.1 \mathrm{~W}$ and $80 \mu \mathrm{m}$ waist $(\Gamma=1.005 \mathrm{MHz}$, $1 \mathrm{~S}-3 \mathrm{~S})$ the count rate is $R_{C W} \approx 10^{-8}$ photons/atom sec. To be more precise, $\Gamma \rho_{e e}$ is the probability of the decay to the ground state per atom per unit of time, since the semi-classical description does not include photons (second quantization). To calculate the experimentally observed count rate, the total number of atoms as well as the collection efficiency and quantum efficiency of the detector must be known.

The excitation can also be driven by two photons from the same field. In this case $\vec{k}_{1}=\vec{k}_{2}$ and the atom sees the Doppler shifted frequency $2 \omega \pm 2 k v_{z}$.

$$
R_{C W}^{D B}=\frac{1}{4}\left[\frac{\Gamma \Omega^{2} / 4}{\left(\omega_{e g}-2 \omega+2 k v_{z}\right)^{2}+\Gamma^{2} / 4}+\frac{\Gamma \Omega^{2} / 4}{\left(\omega_{e g}-2 \omega-2 k v_{z}\right)^{2}+\Gamma^{2} / 4}\right]
$$

There are two Doppler broadened (DB) components, one from each direction of the field. The Doppler-broadened lines are shifted by $2 k v_{z}$ and to obtain the full line shape, one needs to integrate the expressions 2.26 over the velocity distribution $p(v)$. In a gas cell (GC), where all directions are equally probable, the average velocity is zero. Both DB components are thus centered at the resonance frequency, just as the DF line. To obtain the Doppler broadened line shape, we can treat the Lorentzian lines for each velocity class as a delta function, since it is much narrower than the Doppler broadening.

$$
\begin{aligned}
R_{C W, G C}^{D B} & =\int_{-\infty}^{\infty} \frac{1}{2} \frac{2 \pi \Gamma \Omega^{2} / 4}{\Gamma} \delta\left(\Delta \omega \pm 2 k v_{z}\right) \frac{1}{\sqrt{\pi} v_{p}} e^{-v_{z}^{2} / v_{p}^{2}} d v_{z} \\
& =\frac{\sqrt{\pi}}{4} \frac{\Omega^{2}}{v_{p} k} e^{-\Delta \omega^{2} /\left(4 k^{2} v_{p}^{2}\right)}
\end{aligned}
$$


Where $\Delta \omega$ is the detunig from the resonance frequency, $v_{p}=\sqrt{2 k_{B} T / M}$ is the most probable velocity ( $T$ temperature, $k_{B}$ Boltzmann constant and $M$ atomic mass) of the Maxwellian distribution $p(v) \propto v^{2} e^{-v^{2} / v_{p}^{2}}$. The velocity distribution for a single velocity component $p\left(v_{z}\right)=1 /\left(\sqrt{\pi} v_{p}\right) e^{-v_{z}^{2} / v_{p}^{2}}$ is normally distributed. As expected, the DB line is Gaussian line with the amplitude reduced by the factor $\sqrt{\pi} \Gamma / 4 k v_{p}$ as compared to a single velocity class DB line. The width of the Doppler broadened line for the $1 \mathrm{~S}-3 \mathrm{~S}$ transition in hydrogen at $T=7 \mathrm{~K}$ is $\omega_{D}=2 \sqrt{\ln 2} k v_{p} \approx 2 \pi \times 3 \mathrm{GHz}$. Taking eq. 2.25 for the DF line, the contrast between the DF and DB count rates in a gas cell is given by the following ratio (cf. [34]).

$$
C_{C W}^{G C}=\frac{4 k v_{p}}{\sqrt{\pi} \Gamma}
$$

For an experiment with an atomic beam $(\mathrm{AB})$ the situation is quite different in that the average velocity in $z$-direction is nonzero. In this case there are two separate DB lines, centered at $\pm 4 k v_{p}$, symmetrically around the resonance frequency. This significantly reduces the Doppler broadened count rate around the resonance an thus improves the contrast. If the atomic beam is sufficiently collimated and collinear with the laser beam, we can neglect the $x$-, $y$-components and set the $z$-component equal to the modulus of the velocity. The Maxwell velocity distribution for a diffusive beam is $p(v) \propto v_{z}^{3} e^{-v^{2} / v_{p}^{2}}$.

$$
\begin{aligned}
R_{C W, A B}^{D B} & =\sum_{ \pm} \int_{-\infty}^{\infty} \frac{2 \pi \Gamma \Omega^{2} / 4}{4 \Gamma} \delta\left(\Delta \omega \pm 2 k v_{z}\right) H( \pm \Delta \omega) \frac{2}{v_{p}^{4}} v_{z}^{3} e^{-v_{z}^{2} / v_{p}^{2}} d v_{z} \\
& =\frac{\pi \Omega^{2}}{4} \frac{e^{-\Delta \omega^{2} /\left(4 k^{2} v_{p}^{2}\right)} \Delta \omega^{3}}{8 k^{4} v_{p}^{4}}[H(\Delta \omega)-H(-\Delta \omega)]
\end{aligned}
$$

Where $H(\delta \omega)$ is the Heaviside step function. To obtain the full Doppler broadened count rate, the polarization dependent excitation of the other fine- and hyperfine transitions (1S-3S $F=0$ and 1S-3D lines) has to be taken into account, since the Doppler width covers also these transitions. Therefore, the contrast generally is reduced. Figure 2.4 shows for comparison the expected total count rate (DF+DB) for CW spectroscopy in a gas cell, assuming only 1S-3S transition (solid black curve), frequency comb excitation in an atomic beam (dashed black curve, only 1S-3S state, 2 ps comb, steady state solution), frequency comb excitation in an atomic beam (dash-dot black curve, all fine- and hyperfine transitions, experimental detection profile included) and the experimentally observed spectroscopy line (blue solid curve and error bars). Details to the contrast of the frequency comb spectroscopy are explained in the next section and in chapter 3 , table 3.1 . 


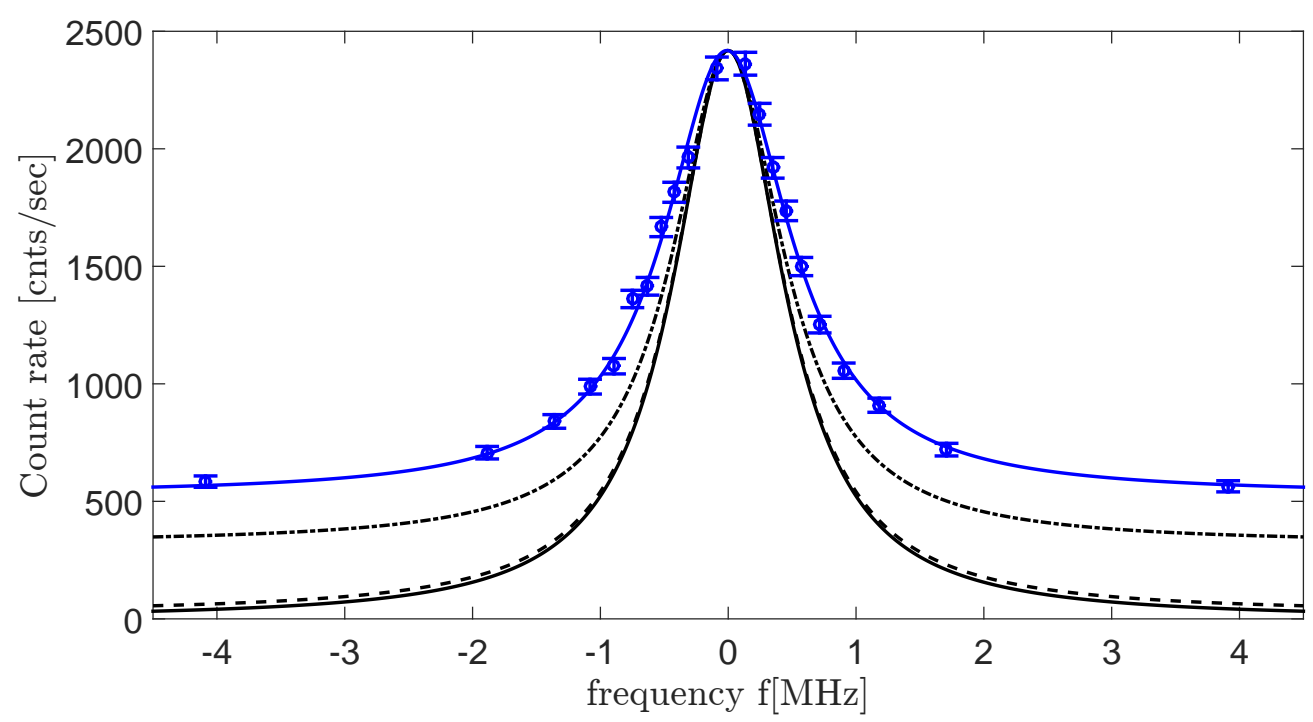

Figure 2.4.: Total count rate $(\mathrm{DF}+\mathrm{DB})$ for $\mathrm{CW}$ spectroscopy in a gas cell, assuming only 1S-3S transition (solid black curve), frequency comb excitation in an atomic beam (dashed black curve, only 1S-3S state, 2 ps comb, steady state solution at center of the pulse collision volume), frequency comb excitation in an atomic beam (dash-dot black curve, all fineand hyperfine transitions, experimental detection profile included) and the experimentally observed spectroscopy line (blue solid curve and error bars). The experimentally detected count rate includes other background counts such as dark count of the PMT, recombination counts and laser scatter (cf. table 3.1)

\subsection{Two-Photon Frequency Comb Spectroscopy}

To derive the steady state solutions for the frequency comb two-photon spectroscopy, we consider two equal counter propagating fields, which have the same carrier frequency $\omega_{0}$ and wave number $k_{0}$ and are polarized along the $z$-axis.

$$
E(t, z)=\frac{1}{2}\left[\vec{E}_{01} \mathcal{E}\left(t+\frac{z}{c}\right) e^{-i\left(\omega_{0} t+k_{0} z\right)}+\vec{E}_{02} \mathcal{E}\left(t+\frac{z}{c}\right) e^{-i\left(\omega_{0} t-k_{0} z\right)}\right]+\text { c.c. , }
$$

with the normalized time-dependent field envelope $\mathcal{E}\left(t \pm \frac{z}{c}\right)$ and electric field vectors $\vec{E}_{0 i}$. For a Gaussian pulse train we obtain:

$$
\mathcal{E}\left(t \pm \frac{z}{c}\right)=\frac{1}{\sqrt{\tau}} \sqrt[4]{\frac{2}{\pi}} \sum_{m=-\infty}^{\infty} e^{-\left(t \pm z / c-n T_{r e p}\right)^{2} / \tau^{2}}
$$

Here $T=2 \pi / \omega_{r}$ is the repetition period. The field $1 / e-$ pulse duration $\tau$ is related to the FWHM intensity pulse duration over $\tau_{1 / 2}=\sqrt{2 \ln (2)} \tau$. We omitted for clarity other possible important characteristics of the pulse such as chirp and the transverse spatial dependence, which easily can be included into a numerical simulation. Here, 
we are interested only in the general features of the solution. The Fourier transform of a Gaussian pulse envelope is given by the following expression.

$$
\tilde{\mathcal{E}}\left(m \omega_{r}\right)=\frac{\sqrt{\tau \omega_{r}}}{\sqrt[4]{2 \pi}} \sum_{m=-\infty}^{\infty} e^{-\left(m \omega_{r} \tau\right)^{2} / 4-i m \omega_{r} z / c} \delta\left(m \omega_{r}\right)
$$

Thus the pulse train can be written as a sum of comb modes.

$$
\begin{aligned}
E(t, z)= & \frac{E_{01} \sqrt{\tau \omega_{r}}}{2 \sqrt[4]{2 \pi}} \sum_{m_{1}=-\infty}^{\infty} e^{-\left(m_{1} \omega_{r} \tau\right)^{2} / 4-i\left(\omega_{0}+m_{1} \omega_{r}\right) t-i k_{0} z-i m_{1} \omega_{r} z / c} \\
& +\frac{E_{02} \sqrt{\tau \omega_{r}}}{2 \sqrt[4]{2 \pi}} \sum_{m_{2}=-\infty}^{\infty} e^{-\left(m_{2} \omega_{r} \tau\right)^{2} / 4-i\left(\omega_{0}+m_{2} \omega_{r}\right) t+i k_{0} z-i m_{2} \omega_{r} z / c}+\text { c.c. }
\end{aligned}
$$

Each pair of modes, which satisfies the resonance condition $\omega_{e g}=2 \omega_{0}+\left(m_{1}+m_{2}\right) \omega_{r}$, contributes to the excitation rate. Due to the periodicity of the frequency comb, the transition will repeat itself for any multiple $\mu \omega_{r}$ of the repetition rate, however with reduced amplitude.

$$
A(\omega) \propto e^{-\left((m-\mu) \omega_{r} \tau\right)^{2} / 2} e^{-\left((m+\mu) \omega_{r} \tau\right)^{2} / 2}=e^{-\left(\mu \omega_{r} \tau\right)^{2}}
$$

Figure 2.5 shows the principle of the two-photon frequency comb spectroscopy. The spectrum of a frequency comb is depicted in the lower plot, with individual mode pairs contributing to the resonance transitions at $\omega_{e g}+\mu \omega_{r}$.

The two-photon time-independent matrix element $\beta_{e g}$ can be easily extended for several different frequencies. For instance, for a frequency comb, $\beta_{g e}$ calculates with the following expression.

$$
\beta_{e g}=\frac{e^{2}}{2 h c \epsilon_{0}} \frac{\tau \omega_{r}}{\sqrt{4 \pi}} \sum_{m=-\infty}^{\infty} \sum_{n} \frac{\langle e|z| n\rangle\langle n|z| e\rangle}{\omega_{n g}-\omega_{0}-\omega_{r} m} e^{-\left(m \omega_{r} \tau\right)^{2} / 2}
$$

Here the sum runs over all mode number $m$. For a narrow frequency comb with spectral width $\left(\omega_{0}+m \omega_{r} \approx \omega_{0}\right)$ much smaller than the carrier frequency $\omega_{0}$, all terms in the sum are approximately equal. For a large number of modes, the sum can be approximated by an integral, such that eq. 2.35 reduces to the simple single frequency expression in eq. 2.15 for $\beta_{e g}$. However care must be taken, if one of the comb modes is close to resonance to any of the intermediate states $|n\rangle$. In this case $\beta_{e g}$ will be different and those terms have to be calculated separately. As explained above, this is not the case for the $1 \mathrm{~S}-3 \mathrm{~S}$ spectroscopy with a picosecond comb. The Rabi frequency $\Omega \propto E_{1}(t, z) E_{2}(t, z)$ depends on the position of the atom. To see this, let the atom's position be at $z$, where zero is the center of the pulse collision volume (PCV), the region where counter propagating pulses overlap (cf. fig. 3.9). For the Rabi frequency, assuming Gaussian pulses, we obtain the following dependence.

$$
\Omega(z) \propto e^{-\left(z^{\prime}-z\right)^{2} /(c \tau)^{2}} e^{-\left(z^{\prime}+z\right)^{2} /(c \tau)^{2}}=\Omega(0) e^{-2 z^{2} /(c \tau)^{2}}
$$




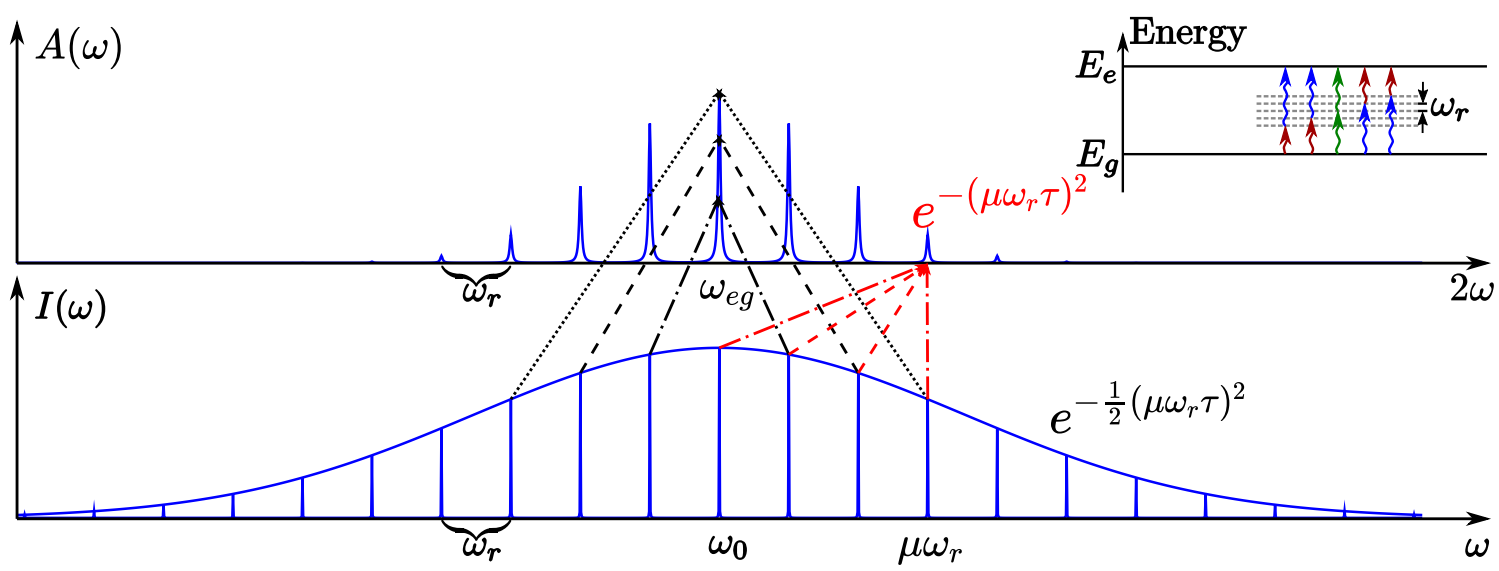

Figure 2.5.: The principle of two-photon frequency spectroscopy is shown. For clarity only one frequency comb spectrum is displayed. All mode pairs, which satisfy the resonance condition, contribute to the total excitation. Due to the periodicity of the frequency comb, transitions appear at multiples of the repetition rate with an amplitude proportional to the square of the comb intensity at this frequency. In the right upper corner, an energy diagram for two-photon frequency comb excitation is shown.

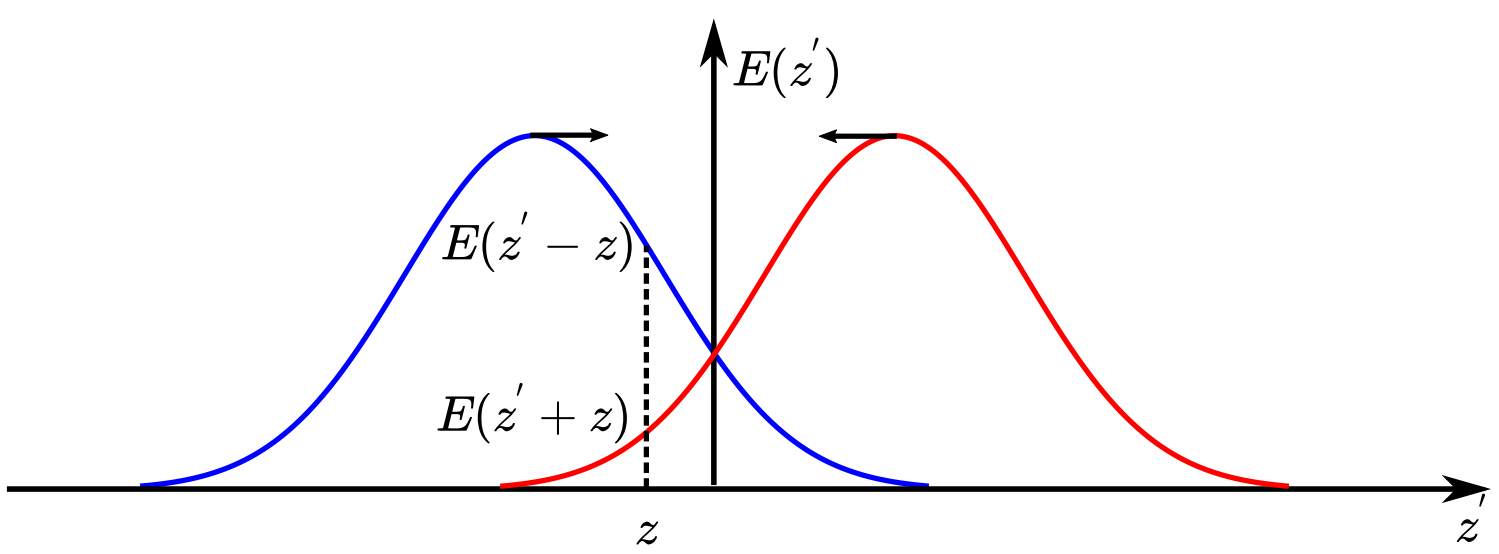

Figure 2.6.: The dependence of the Rabi frequency on the position within the pulse collision volume for two-photon frequency comb spectroscopy is shown. At a position $z^{\prime}=z$ the pulses pass the the atom asymmetrically, leading to a position dependence of $\Omega(z) \propto$ $e^{-2 z^{2} /(c \tau)^{2}}$.

Where $\Omega(0)$ is the Rabi frequency for an atom at the center of the pulse collision volume. Figure 2.6 illustrates the position dependence of the Rabi frequency. The steady state solution can be calculated using the CW solution in eq. 2.25, the linearity of the optical Bloch equations 2.43 and the dependencies on the position of the atom $z$ in eq. 2.36 and the comb envelope detuning eq. 2.34. The Doppler free count rate for two-photon frequency comb excitation is therefore given by the 
following sum.

$$
R_{F C}^{D F}=\frac{\omega_{r} \tau}{\sqrt{4 \pi}} \sum_{-\infty}^{\infty} \frac{e^{-\left(m \omega_{r} \tau\right)^{2} / 2} \Gamma \Omega^{2} / 4}{\left(\omega_{e g}-2 \omega_{0}-2 m \omega_{r} v_{z} / c\right)^{2}+\Gamma^{2} / 4} e^{-4 z_{0}^{2} /(c \tau)^{2}} e^{-\left(\mu \omega_{r} \tau\right)^{2}}
$$

Each resonant pair of modes produces a Doppler shifted Lorentzian line $(\Delta \omega=$ $\left.2 n \omega_{r} v_{z} / c\right)$. However, for any pair of the modes $m=n$ there is always an identical line with $m=-n$, such that the overall line is only Doppler broadened, but there is no net shift. The total broadening is proportional to the width of the frequency comb and is the frequency domain description of the well known Time-of-flight broadening. It amounts to $\Gamma_{T O F}=8 \ln (2) \bar{v} / c \tau$. For a large number of modes the sum is well approximated by an integral over the mode number $m$. This is a convolution between a Lorentzian and a Gaussian, which upon integration results in a Voigt-like line shape. However for cryogenic temperatures and thus slow atoms, we can approximately include the broadening into the natural line width, assuming that the simple Lorentzian line shape is preserved.

$$
R_{F C}^{D F}=\frac{\Gamma \Omega^{2} / 4}{\left(\omega_{e g}-2 \omega_{0}\right)^{2}+\left(\Gamma+\Gamma_{T O F}\right)^{2} / 4} e^{-4 z_{0}^{2} /(c \tau)^{2}} e^{-\left(\mu \omega_{r} \tau\right)^{2}}
$$

Thus, if the Time-of-flight broadening is small compared to the natural line width $\Gamma$ and additionally the central mode is on resonance with the transition frequency $(\mu=0)$ and the atom is at the center of the pulse collision volume $(z=0)$, then the frequency comb two-photon Doppler-free count rate is the same as for the CW spectroscopy with the same average power.

The second order AC Stark shift for two-photon frequency comb excitation is analogous to the CW case in eq. 2.18 given by the following expression.

$$
\Delta E_{A C}^{\phi}(t)=\frac{e^{2}}{\hbar} \frac{\omega_{r} \tau}{\sqrt{4 \pi}} \sum_{m=-\infty}^{\infty} \sum_{n, \pm} \frac{\langle\phi|\hat{z}| n\rangle\langle n|\hat{z}| \phi\rangle}{\omega_{n g} \pm\left(\omega_{0}+m \omega_{r}\right)} e^{-\left(m \omega_{r} \tau\right)^{2} / 2} E_{0}^{2}
$$

It is linear in intensity and if the spectral band width of the comb is small compared to the carrier frequency and no resonant intermediate transitions are present, the sum over the mode number $m$ can be approximated by an integral and we obtain the same AC Stark shift (cf. eq. 2.20) as for the CW spectroscopy with the same average intensity. The fourth order AC Stark shift for the frequency comb case is discussed in section $\mathrm{B}$ and constitutes a negligibly small correction for the 1S-3S comb spectroscopy of this work.

The Doppler-broadened count rate for frequency comb spectroscopy is different than for CW spectroscopy in several aspects. First, the spectral width of a frequency comb is typically much larger than CW Doppler width $(2$ ps comb, $\Delta \omega \approx 2 \pi \times$ $100 \mathrm{GHz} \gg \omega_{D} \approx 2 \pi \times 3 \mathrm{GHz}$ ), such that Doppler broadened line is given by the width of the frequency comb rather than the velocity distribution, as it is the case for the CW spectroscopy. This is advantageous as the Doppler broadened count rate 
is even less frequency dependent, reducing a possible asymmetric shift. Note, that due to the two-photon nature of the transition, the line width is proportional to the square of the intensity and thus the width of the comb reduces by a factor of $\sqrt{2}$, assuming a Gaussian comb. Second, each velocity class is excited by the frequency comb as efficient as with the CW laser, but due to its large spectral width, a comb can simultaneously talk to many velocity classes, thus increasing the Doppler-broadened count rate by approximately the number of modes within the spectral bandwidth $2 \omega_{D} / \omega_{r}$ (roughly 40 for $1 \mathrm{~S}-3 \mathrm{~S}$ transition). Therefore, the contrast of the Doppler-free to Doppler-broadened count rates is approximately given by the following expression.

$$
C_{c o m b}=\frac{4 k v_{p}}{\Gamma \sqrt{\pi}} \frac{\omega_{r}}{2 \omega_{D}}=\frac{\omega_{r}}{\Gamma \sqrt{\pi \ln (2)}}
$$

As in the CW case this equation does not take into the account the polarization dependent excitation of other allowed fine- and hyperfine components, which can also be resonant. Figure 2.4 shows the total theoretical and experimental line shapes of the 1S-3S transition in hydrogen for both $\mathrm{CW}$ and frequency comb excitation, which includes the polarization as well as detector properties $(T=7 \mathrm{~K}$, main detector cf. section 3). Third, since the width of the Doppler broadened line is given by the spectral width of the comb, both DB lines from counter propagating beams will be almost centered at the transition frequency, which is a disadvantage as compared to the CW case, where the use of an atomic beam significantly reduces the Doppler background and thus improves the contrast. Finally, as explained above, in the case of frequency comb excitation, the Doppler-free fluorescence is emitted only within the tiny region, where the pulses overlap. The Doppler-broadened fluorescence is emitted across the entire atomic beam and thus can be collected outside of the pulse collision volume using an independent detector. It than can be used as an almost perfect normalization signal. This is explained in detail in section 4.2. This normalization signal is not present in the CW case, as the Doppler-free and Doppler-broadened fluorescence can not be separated. In the next section, the Monte Carlo simulation for the 1S-3S experiment is explained. 


\subsection{Monte Carlo simulations}

The Monte Carlo simulation has been developed and performed by Arthur Matveev [35]. It has played a crucial role for the development and analysis of this experiment. The Monte Carlo simulation of the experimental 1S-3S line shapes consists of four modules. The first one determines a randomly picked atomic trajectory. The second module solves the two-photon optical Bloch equations for an atom that flies along this trajectory through the pulse collision volume (PCV) for a certain laser detuning, intensity, pulse duration and chirp parameter. The third module determines the spatial fluorescence strength of the Balmer- $\alpha$ fluorescence, that is emitted upon the $3 \mathrm{~S} \rightarrow 2 \mathrm{P}$ decay. The last module determines the line shape, by convoluting the spatial fluorescence strength with the spatial light collection profiles of our four detectors (see section 3.6). To deal with different velocity distributions, we perform the computations for a set of velocities $v_{j}=1 \ldots 10,000 \mathrm{~m} / \mathrm{s}$ in $j=1 \ldots 400$ steps of $25 \mathrm{~m} / \mathrm{s}$. A weighted sum allows us to use any velocity distribution model in postprocessing the simulation results.

To seed the trajectories we first determine a starting point from a uniform distribution on a circular surface within the nozzle that is perpendicular to the atomic and laser beam axis (the $z$-axis). This surface is not necessarily positioned at the nozzle orifice (see section 5.4). Adapted to the experimental set-up, the diameter of this surface is set to $1.5 \mathrm{~mm}$. After the initial point is selected the angle of the trajectory is drawn from a distribution that is proportional to $\cos (\theta) d \Omega_{s}$ [36], where $d \Omega_{s}=|\sin (\theta)| d \theta d \varphi$ and the polar and azimuthal angles $\theta$ and $\varphi$ respectively. The polar angle is measured with respect to the beam axis. To accelerate a computation, the seeding algorithm checks if the trajectory passes through the PCV, and discards the trajectory if this is not the case. The condition is that it crosses an ellipsoid with the semi-axis of $2 w_{0}$ and $2 c T_{1 / 2}$ that is centred at the PVC. We also save the fraction of trajectories that pass through the PCV for absolute calibration of the signal. About 10,000 trajectories are used to sample the line at each of the 21 laser frequencies that are used in the measurement. We use the same set of trajectories for each laser frequency.

To test the dependence of the geometry we have varied the nozzle diameter and even used an annual shape to model atoms that are directly emitted from the inner surfaces of the nozzle. We find very little variation of the resulting centre frequencies with these alterations. The accuracy of the Monte Carlo simulation is given by the typical variation of the centre frequencies between the trajectories divided by the square root of the number of trajectories. The variation is mostly determined by the variation of AC-Stark shift, since the SOD is constant for a fixed velocity. Therefore we can determine the line centres within a few Hz. We have verified this accuracy by comparing runs with different sets of trajectories. When comparing simulations where we artificially turn on and off some effects as described in section 5.11, we expect an even smaller uncertainty, because the noise introduced through the choice of the trajectories is common mode, at least when it is possible to compare runs with 


\section{Two-photon Direct Frequency Comb Spectroscopy}

the same trajectory sets.

Including collisions into the Monte Carlo simulation would be possible. Collisions with large impact parameter (Weisskopf radius) lead to phase shifts and hence a frequency shift of the line, while the a small impact parameter resets the off-diagonal matrix elements and leads to a collisional broadening (and possibly a new trajectory). To model all this would require two more random variables, the impact parameter and the relative velocity of the colliding atoms or molecules. In addition the chemical composition, i.e. the hydrogen dissociation fraction depends on temperature (see fig.3.8). Hence including the pressure shift into our Monte Carlo simulation would significantly increase the computational effort that is already considerable. In light of the smallness of the pressure shift we have not made an attempt in modelling it this way.

To describe the evolution of the atomic state we use the optical Bloch equations (OBEs). In most of our simulations we represent the hydrogen atom as a two-level system with the ground state $g=|1 \mathrm{~S}\rangle$ and the excited state $e=|3 \mathrm{~S}\rangle$. The OBEs for a two-photon transition are identical to the one-photon case, except that the Rabi frequency is computed differently. With the rotating wave approximation, they can be expressed in terms of the density matrix:

$$
\begin{aligned}
& \dot{\rho}_{g g}=-i \frac{\Omega^{*}(t)}{2} \rho_{e g}+i \frac{\Omega(t)}{2} \rho_{g e}+\Gamma \rho_{e e} \\
& \dot{\rho}_{g e}=-i \Delta \omega \rho_{g e}+i \frac{\Omega^{*}(t)}{2}\left(\rho_{g g}-\rho_{e e}\right)-\frac{1}{2} \Gamma \rho_{g e} \\
& \dot{\rho}_{e e}=+i \frac{\Omega^{*}(t)}{2} \rho_{e g}-i \frac{\Omega(t)}{2} \rho_{g e}-\Gamma \rho_{e e}
\end{aligned}
$$

The equation for $\dot{\rho}_{e g}$ is obtained as the complex conjugate of the second equation. Here $\Omega(t)$ is the Rabi frequency that is given in (5.2) but deprived of the carrier frequency $-2 \omega_{c}$, because of the rotating wave approximation. For chirped pulses the Rabi frequency becomes complex in contrast to the two-photon OBEs in [30]. The $3 \mathrm{~S} \rightarrow 2 \mathrm{P}$ decay rate is given by $\Gamma / 2 \pi=1.0050 \mathrm{MHz}$ and the laser detuning by $\Delta \omega$. Photoionization of the excited state is possible with just one additional laser photon and might be included into the OBEs. However, for the power levels in use, this results in a sub- $\mathrm{Hz}$ line shift so that we ignore ionization here. The second order Doppler and AC-Stark (see (5.13)) shifts are readily included into the OBEs by subtracting them from the laser detuning:

$$
\Delta \omega=2 \pi\left(\nu-\nu_{0}\right)+2 \pi \nu_{0} \frac{v^{2}}{2 c^{2}}-\delta \nu_{A C}
$$

As in (4.1), the laser frequency and the unperturbed transition frequency are given by $\nu$ and $\nu_{0}$ respectively. In the simulations we usually set $\nu_{0}=0$, since we are only interested in relative line shifts. After solving the OBEs as a function of time, the fluorescence signal emitted between $t_{k}$ and $t_{k+1}$ computes as:

$$
s_{k}\left(\nu, v_{j}\right)=\Gamma \int_{t_{k}}^{t_{k+1}} \rho_{e e}(t) d t .
$$


Knowing the position of the atom as a function of time, we can determine the spatial fluorescence strength. As $s_{k}\left(\nu, v_{j}\right)$ increases by unity for one decay event, the unit of $s_{k}\left(\nu, v_{j}\right)$ is photons, even though the semi-classical OBEs actually do not know about photons. The Rabi frequency parameter $\beta_{g e}$ is given in [30] for linear laser polarisation. Converting to $\sigma_{+}$and $\sigma_{-}$polarisations, this parameter becomes $\beta_{g e}=-1.00333 \times 10^{-5} \mathrm{~Hz} /\left(\mathrm{W} / \mathrm{m}^{2}\right)$ and $\beta_{g e}=-3.082895 \times 10^{-5} \mathrm{~Hz} /\left(\mathrm{W} / \mathrm{m}^{2}\right)$ for the $1 \mathrm{~S}-3 \mathrm{~S}$ and $1 \mathrm{~S}-3 \mathrm{D}$ transition respectively. The latter coefficient is has to be multiplied by $+1 / 2$ relative the value given in [30]. The latter is required to model the quantum interference (see section 5.8), for which we need to expand the OBEs to include the $\mathrm{D}$-levels, the final $\mathrm{P}$-levels, their fine- and hyperfine structure as well as all corresponding Zeeman levels. The resulting OBEs consist of 1600 coupled differential equations that are derived using a computer algebra system and numerical integrated on a large scale cluster computer [35].

Since the laser beam radius near the PCV is much smaller than the depth of focus and the resolution of our light collection optics, we do not save the $x$ and $y$ coordinates of the spatial fluorescence strength. Instead the integration code splits the integration interval from $z_{k}=-2 \mathrm{~mm}$ to $z_{k}=+2 \mathrm{~mm}$ into $k=1 \ldots 400$ slices of $10 \mu \mathrm{m}$ each. The times $t_{k}$ in (2.45) are the entrance times into the $k$-th slice of the trajectory under consideration. Finally, to obtain the simulated line shapes as observed with the various detectors, we are convoluting the spatial light collection profiles $p_{D}(z)$ with the spatial fluorescence strength. We determine $p_{D}(z)$ with a Monte Carlo method by drawing a position within the PCV and an emission direction from an isotropic distribution. Following this ray determines whether or not it hits one of the light collecting lenses (see section 3.6). If it does, we compute the refraction through the lens and determine whether it ends up within the acceptance cone of the multi-mode fibre behind the lens. The light collection profiles obtained in this way are shown in fig.3.10. Finally the simulated line shape is obtained via:

$$
\mathscr{L}_{M C}(\nu, z)=\sum_{j, k} s_{k}\left(\nu, v_{j}\right) p\left(v_{j}\right) p_{D}\left(z_{k}-z_{D}\right)
$$

where $z_{D}$ is the central position of the light collecting lens and $p\left(v_{j}\right)$ is the normalized atomic velocity distribution. Examples for light collection profiles $p_{D}(z)$ and fitting results to $\mathscr{L}_{M C}(\nu, z)$ are seen in fig.5.2. 



\section{Experimental setup}

In this chapter the experimental setup is presented. Fig. 3.1 shows the general scheme of the $1 \mathrm{~S}-3 \mathrm{~S}$ experiment. In the following sections the individual parts are explained. In section 3.1 the absolute frequency determination is described (left side of fig. 3.1). Section 3.2 deals with the frequency quadrupling to obtain $205 \mathrm{~nm}$ frequency comb for spectroscopy and explains the chirp generation mechanism via self-phase modulation process in the second harmonic generation cavities (right side in fig. 3.1, compare fig. 3.3, 3.4,). In section 3.4 the vacuum system with the enhancement cavity and differential pumping to prevent mirror degradation are explained (compare fig. 3.6, 3.6). Section 3.5 deals with the hydrogen discharge and the liquid helium cryostat cooling (fig. 3.7, 3.8). Finally, section 3.6 presents the details of the fluorescence detection (fig. 3.9, 3.10).

\subsection{Frequency measurement}

As shown in Fig. 3.1, we operate two frequency combs at $820 \mathrm{~nm}$ - one generated by a femtosecond laser ("fs-comb") to determine the optical frequency of the 1S3S transition, and one from a picosecond laser ("ps-comb") for direct frequency comb spectroscopy. The ps-comb is obtained with a mode-locked Ti:sapphire laser (Spectra Physics, Tsunami) and the fs-comb is generated with a frequency doubled mode-locked erbium-doped fiber laser (Menlo Systems, FC1500). The ps-comb is calibrated by the fs-comb in two steps. First, we measure the reference frequency of a continuous wave (cw) extended cavity diode laser (ECDL) at $820 \mathrm{~nm}$ that is locked to an ultra stable cavity with the Pound-Drever-Hall technique:

$$
f_{\mathrm{ref}}(820 \mathrm{~nm})=(1461379 \times 250) \mathrm{MHz}+60 \mathrm{MHz}-150 \mathrm{MHz}+\left\langle f_{b}\right\rangle .
$$

The repetition and carrier-envelope offset frequencies of the fs-comb are set to $250 \mathrm{MHz}$ and $60 \mathrm{MHz}$ respectively using a GPS disciplined hydrogen maser as a reference. The calibration uncertainty contributes $30 \mathrm{~Hz}$ to the absolute frequency (see table 5.1). The $150 \mathrm{MHz}$ frequency offset is obtained through mixers to obtain more convenient frequencies for counting. The beat note frequency $f_{b}$ of the $\mathrm{cw}$ laser with the 1461379 -th mode of the fs-comb is recorded redundantly with two $\Lambda$-type counters [51] (K+K Messtechnik, FX80), and is stable enough so that is treated as a constant during a line scan that takes $\approx 36$ sec. By using a line-scan-averaged value $\left\langle f_{b}\right\rangle$ we effectively remove residual noise of the hydrogen maser that is much larger than the cavity-locked $\mathrm{cw}$ laser at this time scale. Without using the ultra stable cavity as a flywheel, the 1S-3S lines would look considerably more noisy. 


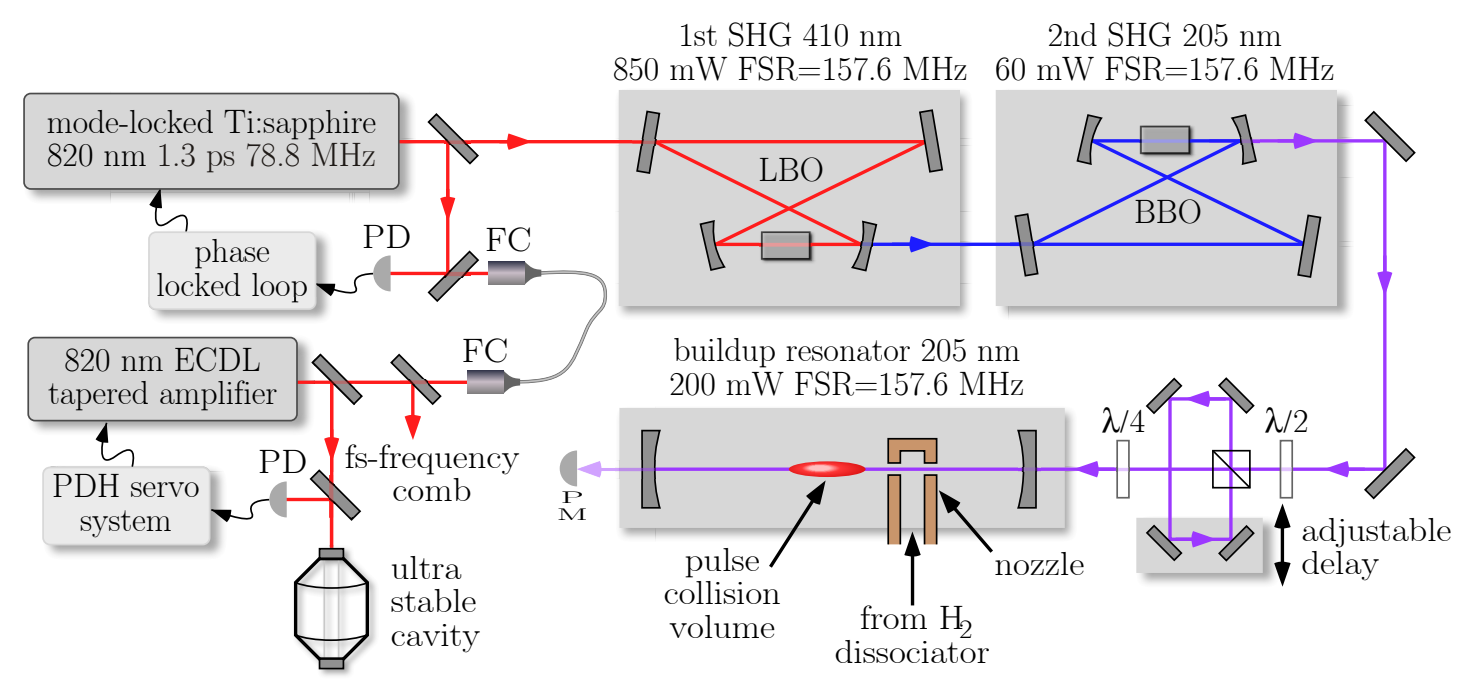

Figure 3.1.: Experimental set-up. The carrier frequency of a mode-locked titanium:sapphire $(78.8 \mathrm{MHz}, 1.3 \mathrm{ps}, 2.8 \mathrm{~W})$ is doubled in an enhancement resonator with half the optical length of the laser resonator. This also doubles the repetition rate. After a second doubling stage, that leaves the repetition rate constant, we obtain $60 \mathrm{~mW}$ of 205-nm radiation with a pulse duration (FWHM) of 2.0 ps [41]. A delay line generates $\sigma^{+} / \sigma^{-}$circular polarised double pulses that meet twice per round trip in the center of the enhancement cavity that is housed in a vacuum chamber. This doubles the repetition rate once more to $315.2 \mathrm{MHz}$ in order to allow using a two-mirror resonator with a sufficiently strong focus. The choice of the polarisation avoids losing half the power in the delay line and reduces the excitation of the $1 \mathrm{~S}-3 \mathrm{D}$ transitions with respect to the $1 \mathrm{~S}-3 \mathrm{~S}$ transitions. The time averaged circulating power in this cavity is about $60 \mathrm{~mW}$ per direction with a focus radius of $w_{0}=80 \mu \mathrm{m}$. Hydrogen atoms are produced by dissociation of hydrogen molecules in a radio frequency discharge tube and are then guided through a teflon tube to a cryogenic T-shaped copper nozzle where they thermalize and escape into the vacuum through two holes. Some of the atoms make it into the cigar shaped pulse collision volume (PCV) and get excited to the 3S state from which they decay within $0.16 \mu$ s to the $2 \mathrm{P}$ state releasing a $656 \mathrm{~nm}$ Balmer- $\alpha$ photon which is detected (see fig.3.9). PM: power meter; FC: fibre coupler; FSR: free spectral range; PDH: Pound-Drever-Hall stabilisation [39]; ECDL: extended cavity laser diode; SHG: second harmonic generation; LBO/BBO: Lithium triborate and $\beta$-barium borate crystals; PD: photo detector.

In a second step, we lock the mode number $n$ of the ps-comb by stabilizing its beat note with the $\mathrm{cw}$ laser to a predefined radio frequency $f_{\mathrm{LO}}$ that is derived from a synthesizer referenced to the hydrogen maser (the "local oscillator"). A piezo mounted mirror of the ps-laser serves as the actuator. As shown in Fig. 3.1, the ps-comb is frequency doubled twice and then drives the 1S-3S two-photon transition (more precisely, all sum frequencies of all modes are generated (see section 3.3) so that frequency doubling alone does not affect the mode spacing $f_{\text {rep }}$ ). Hence, there 
is a factor of 8 relating the frequency of the reference laser to the atomic frequency (measured at $102.5 \mathrm{~nm}$ ):

$$
f_{1 \mathrm{~S}-3 \mathrm{~S}}(102.5 \mathrm{~nm})=8\left(f_{\mathrm{ref}}(820 \mathrm{~nm})+f_{\mathrm{LO}}+\frac{n}{2} f_{\mathrm{red}, \mathrm{ps}}\right) .
$$

The repetition frequency of the ps-comb $f_{\text {rep,ps }}$ is free running at around $78.8 \mathrm{MHz}$ and is recorded along with $f_{b}$ so that the spectroscopy laser frequency is determined at any time during the line scans. Here, $n$ is not the mode number, but the halfmode number [25]. In contrast to the fs-comb, we number the modes of the ps-comb starting with the mode that is locked to the cw reference laser. The mode number that is closest to the actual transition is $n=-47$, as derived from comparisons with theoretical predictions. Note that these predictions are more than four orders of magnitude more accurate than required to identify the mode number. By varying $f_{\mathrm{LO}}$ we scan the ps-comb across the line.

In order to detect and remove occasional glitches of the phase locked loops, we employ selection criteria. The beat note frequency $f_{b}$ is redundantly measured with two counters. Data points where the readings are off by more than $0.2 \mathrm{~Hz}$ are not taken into account for computing the average $\left\langle f_{b}\right\rangle$. In the same way we proceed with readings of the repetition and carrier-envelope offset frequencies of the fs-comb that are off from their local oscillators by more than $10 \mathrm{mHz}$ and $0.5 \mathrm{~Hz}$ respectively. Since the stabilization of the reference laser operates essentially without glitches, removing these frequency readings only affects the statistics of $\left\langle f_{b}\right\rangle$, but otherwise has no effect on the spectroscopy.

An additional cycle slip detection is implemented for monitoring $f_{\mathrm{LO}}$. If this frequency deviates by more than $0.5 \mathrm{~Hz}$ from the set local oscillator frequency we discard the respective data point from the line scan. The cycle-slip thresholds are chosen by adapting to the observed stabilities of the monitored frequencies. We have verified that varying the thresholds within reasonable limits has no effect on the final result. Additional cuts are described in section 4.1. 


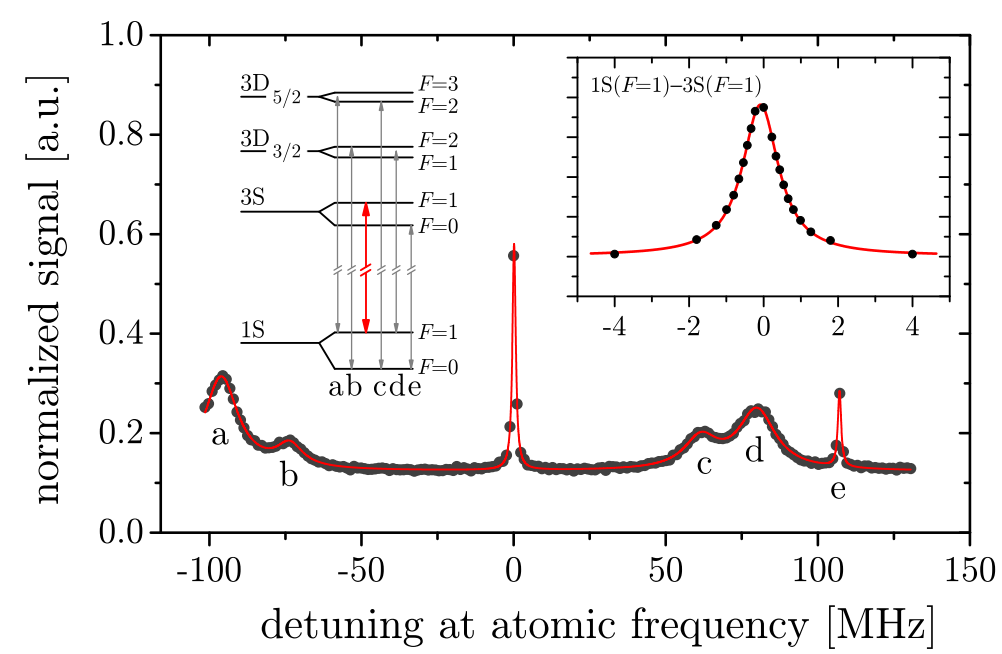

Figure 3.2.: Multiple 1S-3S/D two-photon transitions fold into the spectrum due to the frequency comb so that the separation of the line components appear modulo $\omega_{r}=2 \pi \times$ $315.2 \mathrm{MHz}$. By operating a well chosen $\omega_{r}$ and with circular polarization, we obtain a well-isolated $1 \mathrm{~S}(F=1)-3 \mathrm{~S}(F=1)$ component. Other components that are allowed by the selection rules are a: $1 \mathrm{~S}(F=1)-3 \mathrm{D}_{5 / 2} ; \mathrm{b}: 1 \mathrm{~S}(F=0)-3 \mathrm{D}_{3 / 2}(F=2)$; c: $1 \mathrm{~S}(F=0)$ $3 \mathrm{D}_{5 / 2}(F=2)$; d: $1 \mathrm{~S}(F=1)-3 \mathrm{D}_{3 / 2}$; e: $1 \mathrm{~S}(F=0)-3 \mathrm{~S}(F=0)$. The hyperfine splitting of the $3 \mathrm{D}$ states is smaller than the natural line width and thus unresolved. The baseline offset is from the Doppler-broadened absorption of two co-propagating photons. The inset shows an average of 5 lines scans $(3 \mathrm{~min})$ within $\pm 4 \mathrm{MHz}$ of the main component, normalized to the Doppler-broadened signal, together with a Lorentzian fit. The sampling of the frequency axis was chosen to yield an approximately equidistant spacing on the signal axis in order to maximize the sensitivity to the center frequency per unit of measurement time. The line pulling by other 1S-3D lines and others (not visible) is negligible and discussed in detail in the supplement. The detuning in this graph is measured at the atomic frequency, i.e. around the frequency given in (6.1).

\subsection{Laser system}

The laser system has been evolved only slightly since it has been described in detail [41]. Driving the 1S-3S Doppler free two-photon transition requires two counter propagating photons at $205.145 \mathrm{~nm}$, that are generated as the fourth harmonic in two subsequent doubling cavities. With two-photon direct frequency comb spectroscopy all fine- and hyperfine components are folded into the recorded spectrum with modulo of half the mode spacing (repetition rate) of the comb. Fig. 3.2 shows and explains the resulting full frequency scan pattern.

To prevent other components from overlapping spectrally with the component of interest, it is desirable to operate at a sufficiently large repetition rate and to choose its exact value wisely. The repetition rate of $78.8 \mathrm{MHz}$ of our ps-laser is too low for this purpose. Conventional frequency doubling in nonlinear crystals does not double the repetition rate because this process actually generates sum frequencies 
of all possible combination of modes. Nevertheless, we can double the repetition rate by employing an enhancement cavity with a free spectral range that is twice the repetition rate of the ps-laser. Both of our doubling cavities have a free spectral range of around $2 \times 78.8 \mathrm{MHz}=157.6 \mathrm{MHz}$. As a result, we use only half of the laser power (every second mode). As shown in Fig. 3.1, a combination of wave plates, beam splitters and a delay line generates $\sigma_{ \pm}$circular polarized double pulses, without further loss of laser power. This doubles the pulse repetition rate as seen by the atoms once more to $f_{\text {rep }}=4 \times f_{\text {rep,ps }}=315.2 \mathrm{MHz}$. This specific value isolates the $1 \mathrm{~S}(F=1)-3 \mathrm{~S}(F=1)$ component to minimize line pullings, i.e. apparent shifts of the extracted center frequency of the main resonance due to the proximity of other resonances (see section 5.9). To obtain the real unfolded line separation of components a), b), c), d) and e) from Fig. 3.2, one needs to add 13, 14, 17, 9 and 4 times $315.2 \mathrm{MHz}$ respectively. To prevent other components from overlapping spectrally with the component of interest, it is desirable to operate at a sufficiently large repetition rate and to choose its exact value wisely. The repetition rate of $78.8 \mathrm{MHz}$ of our ps-laser is too low for this purpose. Conventional frequency doubling in nonlinear crystals does not double the repetition rate because this process actually generates sum frequencies of all possible combination of modes. Nevertheless, we can double the repetition rate by employing an enhancement cavity with a free spectral range that is twice the repetition rate of the ps-laser. Both of our doubling cavities have a free spectral range of around $2 \times 78.8 \mathrm{MHz}=157.6 \mathrm{MHz}$. As a result, we use only half of the laser power (every second mode). As shown in Fig. 3.1, a combination of wave plates, beam splitters and a delay line generates $\sigma_{ \pm}$circular polarized double pulses, without further loss of laser power. This doubles the pulse repetition rate as seen by the atoms once more to $f_{\text {rep }}=4 \times f_{\text {rep,ps }}=315.2 \mathrm{MHz}$. This specific value isolates the $1 \mathrm{~S}(F=1)-3 \mathrm{~S}(F=1)$ component to minimize line pullings, i.e. apparent shifts of the extracted center frequency of the main resonance due to the proximity of other resonances (see section 5.9). To obtain the real unfolded line separation of components a), b), c), d) and e) from Fig. 3.2, one needs to add 13, 14, 17, 9 and 4 times $315.2 \mathrm{MHz}$ respectively.

The first frequency doubling stage uses a $5 \mathrm{~mm}$ long lithium triborate (LBO) crystal (cut at $\theta=90^{\circ}, \phi=29.7^{\circ}$ ). It is used within a four mirror bowtie ring cavity with two curved mirrors $\left(\mathrm{ROC}=210 \mathrm{~mm}\right.$, waist radius $\left.w_{0}=40 \mu \mathrm{m}\right)$. The input power from the laser is around $2.8 \mathrm{~W}$ and the single pass efficiency is around $5-10 \%$, allowing for a low finesse cavity (input coupler transmission $T=30 \%$ ). The typical output power at the second harmonic is $800 \mathrm{~mW}$ which corresponds to an efficiency of almost $60 \%$ for the laser modes that are actually used. One of the flat mirrors is mounted on a piezo transducer to stabilize the cavity with respect to the laser using the polarization method [52].

The second doubling stage uses the same type of four mirror ring cavity (ROC $=210 \mathrm{~mm}$, waist $w_{0}=30 \mu \mathrm{m}, T=30 \%$ ) with a $5 \mathrm{~mm}$ barium borate (BBO, $\theta=85.7)$ crystal. The crystal is cooled to $-20^{\circ} \mathrm{C}$ to increase its conversion coefficient $\left(d_{e f f}=0.318 \mathrm{pm} / \mathrm{V}\right)$ and is flushed with dry nitrogen during operation to prevent 


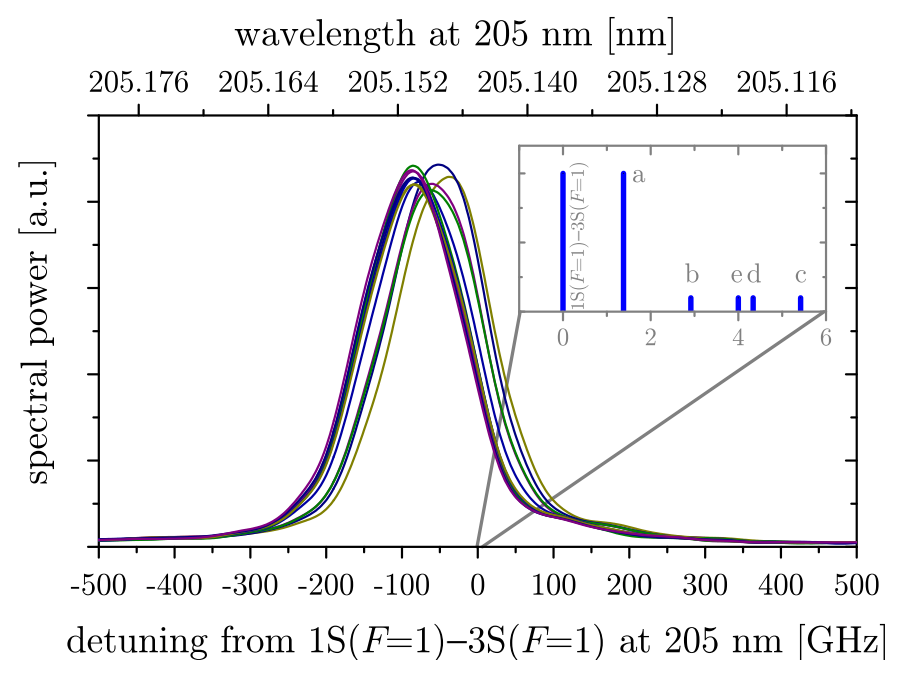

Figure 3.3.: Several measured spectra of the $205 \mathrm{~nm}$ frequency comb recorded within $2 \mathrm{~min}$ and a resolution of $70 \mathrm{GHz}$. The spectral envelope of the comb is jittering significantly. Since the underlying comb is not following the jitter, it gives rise mostly to amplitude noise and much less to frequency noise. Still this jitter is one of the main noise contributions besides shot noise (see section 4.2). Superimposing and averaging about 20,000 of these spectra we find an approximate $\mathrm{Sech}^{2}$ shape with a FWHM of $148 \mathrm{GHz}$ (after correcting for the spectral resolution) which would correspond in the time domain to a Fourier limited pulse duration of $T_{1 / 2}=2.13 \mathrm{ps}(\mathrm{FWHM})$. In the following we are also using the $1 / e$ half-width $\tau=T_{1 / 2} / \sqrt{2 \ln (2)}$. Taking the average chirp induced spectral broadening of $\sqrt{1+b^{2}}$ (see Fig. 5.6) into account slightly reduces the pulse duration. For the Monte Carlo simulations described in section 2.5 , we use $T_{1 / 2}=2.0 \mathrm{ps}$. The inset shows the fine and hyperfine components with their respective line strengths for the given polarization and with labels as shown in Fig. 3.2 (the different order there is due to the convolution with the comb). During the measurement, these components where not always centered on the comb envelope like in this example. We readjusted the spectral envelope occasionally during the measurement to maintain the signal strength. More data on the laser system can be found in [41].

water condensation. With this cavity we obtain $50 \mathrm{~mW}$ of time-averaged power and pulses of $T_{1 / 2}=2.0 \mathrm{ps}$ duration $(\mathrm{FWHM})$. The corresponding $205 \mathrm{~nm}$ frequency comb is $148 \mathrm{GHz}$ (470 modes) wide (FWHM) as shown in Fig. 3.3. Unfortunately this power level reduces within minutes after locking the cavity by approximately a factor of two where it typically stabilizes. This is attributed to a non-permanent degradation of the BBO crystal. The output power can be restored by moving the crystal transversally while the cavity is locked. We believe that similar instabilities are the cause of the significant jitter of the comb envelope that we do not see at the fundamental laser frequency. In addition to these instabilities, self-phase modulation within the crystal of the second doubling stage gives rise to the largest contribution to the pulse chirp (see section 5.1 for details). 
As shown in Fig. 3.1, the doubling stages are followed by a polarizing beam splitter and a delay line at half the cavity's round trip length. Before that, the polarization is rotated by $45^{\circ}$, such that the power is split equally. After the delay line the polarization is converted to $\sigma_{ \pm}$polarization using a $\lambda / 4$ wave plate. A small fraction of both beams that is reflected from the AR coated surface of the $\lambda / 4$ wave plate is guided to a photo diode so that interference fringes can be registered when sweeping the delay line. We overlap the pulses temporally to within $\pm 40 \mu \mathrm{m}$ by maximizing the contrast of these fringes. With this we obtain a single region where the counter-propagating pulses collide, the pulse collision volume (PCV). With a misaligned delay line we would have two PCVs instead. The $\sigma_{+}$and $\sigma_{-}$polarization carries angular momentum so that the 1S-3S transition is forbidden when absorbing two photons from the same direction. This significantly reduces the Doppler-broadened background (see section 3.6). The atoms that fly through the PCV experience a linearly polarized laser field at each position. The polarization direction alternates between the pulses as the direction of the $\sigma_{+}$and $\sigma_{-}$pulses also alternates.

One of the main systematic frequency shifts for the hydrogen 1S-3S direct frequency comb spectroscopy is due to the chirp of the laser pulses - a frequency sweep across the pulses that is periodic with the pulse train. As detailed in section 5.1, this can lead to a so-called chirp induced first-order Doppler shift (CIFODS) if the atomic beam is divergent. Here we explain the main source of the chirp and how it can be varied.

In general, pulses that propagate in media are subject to broadening - both temporally and spectrally. The former is caused by group velocity dispersion (GVD) and typically dominates for fs-pulses while the latter is caused by self-phase modulation (SPM) and typically dominates for ps-pulses. Both types of broadening impose a chirp on an initially unchirped pulse. The characteristic dispersion length [75], $L_{D}=T_{1 / 2}^{2} / \mathrm{GVD}$ in BBO, is about $20 \mathrm{~m}\left(\mathrm{GVD}=200 \mathrm{fs}^{2} / \mathrm{mm}\right.$ at $410 \mathrm{~nm}$ with comparable values for the other involved materials and wavelengths) and thus dispersion effects are negligible in our case. Dielectric mirrors may contribute with tens to hundreds of $\mathrm{fs}^{2}$, which is also negligible for ps-pulses. Self-phase modulation is caused by a small intensity-dependent part of the refractive index, expressed as $n=n_{0}+n_{2} I(z, t)$, where $n_{0}$ is the usual linear index of refraction and $n_{2}$ is the nonlinear index of refraction. The latter only becomes important with high intensity $I(z, t)$, which is the case for the fundamental beams within the crystals of our second harmonic generation cavities. Multiple passes through the crystals further enhance this effect [76]. The total nonlinear temporal phase acquired by the pulses upon propagation though a crystal of the length $L$ can be estimated through the so-called $B$ integral:

$$
\phi_{N L}(t)=\frac{2 \pi}{\lambda} \int_{0}^{L} n_{2} I(z, t) d z,
$$

where we use the on-axis intensity. Both, for BBO and LBO crystals, the nonlinear refractive index is about $n_{2} \approx 4 \times 10^{-16} \mathrm{~cm}^{2} / \mathrm{W}$. With the typical operational pa- 
rameters of the second doubling cavity of $T_{1 / 2}=1.1 \mathrm{ps}$ at $410 \mathrm{~nm}[41], P_{0}=4.8 \mathrm{~W}$ average power, and $157.6 \mathrm{MHz}$ repetition rate, we obtain a peak intensity of up to $I_{0}=(2 / \pi)^{3 / 2} P_{0} \sqrt{2 \ln (2)} / T_{1 / 2}\left(2 f_{\text {rep }}\right) w_{0}^{2}=0.47 \mathrm{GW} / \mathrm{cm}^{2}$. For a $5 \mathrm{~mm}$ long crystal this corresponds to only 14 mrad phase difference across the pulse duration. However, the phase due to multiple passes through the crystal must be summed.

To estimate the total resulting chirp, we use a simple numeric model: an initially unchirped Gaussian pulse is transmitted through the input mirror with transmittance $T$; the pulse is then damped by the linear cavity losses $L=1-T$ (assuming impedance matching); self-phase modulated is realized by imposing the phase $\phi_{N L}(t)$; finally the altered pulse is coherently added to the next impinging pulse at the input coupler. This process is iterated until steady state is reached. Figure 3.4 shows the resulting total nonlinear phase for the second doubling cavity with the doubling crystal centered with respect to the cavity focus and displaced longitudinally by $2 \mathrm{~mm}$. In this way we estimate the range of the chirp parameter to be $b=-0.44 \ldots-0.17$ (see section 5.1 for the definition of $b$ ). Ideal frequency doubling leaves the chirp parameter unaffected and merely reduces the pulse duration by a factor of $\sqrt{2}$. Dispersion and the crystal phase matching bandwidth are not limiting in our experiment, and we expect to operate close to this ideal case. We shift the crystal of the second doubling stage longitudinally to vary the chirp parameter experimentally while the first doubling stage operates stably so that we do not touch it during the measurement. Its contribution to the chirp parameter is therefore fixed within a measurement day.

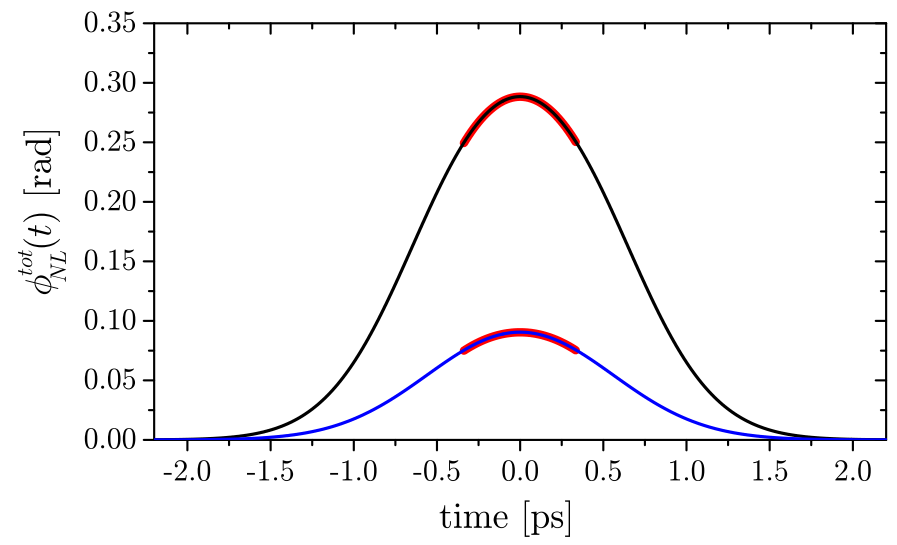

Figure 3.4.: Simulation of self-phase modulation of a pulse circulating inside of the second doubling cavity. The resulting phase across the pulse varies like the intensity envelope. Depending on the alignment of the cavity and by intentionally shifting the crystal longitudinally by about $2 \mathrm{~mm}$ we vary the beam radius within the crystal roughly between $w=20 \mu \mathrm{m}$ and $w=40 \mu \mathrm{m}$. The red curves are parabolic fits within \pm 0.33 ps to extract the chirp parameters of $b=-0.44$ and $b=-0.17$.

In addition to the doubling cavities, the mode locked laser itself is an additional source of chirp. The tight focus inside the Ti:sapphire crystal gives rise to SPM, 
which in this laser (Spectra Physics, Tsunami) is compensated with an intra-cavity Gires-Tournois interferometer. The length of this interferometer is controlled via a piezo transducer. Adjusting its voltage also varies the chirp parameter. However, this contribution to the chirp depends on other alignment parameters of the laser and is difficult to quantify. Like the first doubling stage, the laser operates stably and is not often realigned. To first-order, the chirp parameters from the different sources add. Experimentally we observe chirp parameter variations of $\Delta b=0.38$ (see see Fig. 5.5), which is somewhat larger than that obtained by modeling the contribution of only one doubling cavity. We believe this is because the contributions from both doubling cavities add to the initial chirp from the laser. Moreover, the experimental parameters may not be exactly the ones used in the model and vary with the alignment of the setup. For the data analysis we do not rely on knowledge of the chirp parameter. It is sufficient to be able to modify it (see section 5.1).

It should be noted that it is not an easy task to measure a chirp described above for ps-pulses, since most phase/amplitude determining devices (such as FROG, SPIDER) rely on the measurement of the (narrow) spectral width. Chirps due to SPM lead to a spectral broadening by a factor of about $\sqrt{1+b^{2}}$ (i.e. by roughly $1 \%$ to $10 \%$ in our case), which is in the range of the observational broadening due to the finite spectral resolution of the spectrometer. 


\subsection{Mode suppression}

The doubling cavities, as described in the previous section, are designed to not only double the optical carrier frequency, but also the repetition rate of the laser. This process is not perfect, and we are left with residual "unwanted" modes from the laser that are not resonantly coupled into the doubling cavities. In an empty cavity, the suppression of the unwanted modes is quite effective because the resonant modes are enhanced while the non-resonant modes are attenuated - even with a low finesse. With several cavities in series one expects a rather good suppression. However, the nonlinear crystals remix the modes and compromise the suppression ratio from what is naively expected.

To estimate the strength of the unwanted modes, we use a simple model and assume a frequency comb of $N$ modes that operates with a repetition rate of $\omega_{r}=$ $2 \pi f_{\text {rep }}$ and has a flat spectral envelope. The field coupled to the first doubling cavity is proportional to:

$$
E(t) \propto \sum_{n} E_{n} e^{i n \omega_{r} t} \quad \text { with } \quad E_{n}= \begin{cases}\sqrt{T} & \text { for } n \text { odd } \\ 1 / \sqrt{T} & \text { for } n \text { even }\end{cases}
$$

Here we assume that the impedance-matched even modes are resonant so that their fields are enhanced by $1 / \sqrt{T}$ with the input coupler power transmission $T$ given in the previous section. The fields of the off-resonant odd modes are suppressed by the inverse factor. The second harmonic field is proportional to the square of the fundamental field, i.e.:

$$
E^{2}(t) \propto \sum_{n, n^{\prime}} E_{n} E_{n^{\prime}} e^{i\left(n+n^{\prime}\right) \omega_{r} t} \quad \text { with } \quad E_{n} E_{n^{\prime}}= \begin{cases}1 & \text { for } n+n^{\prime} \text { odd } \\ 1 / T & \text { for } n \text { and } n^{\prime} \text { even } \\ T \approx 0 & \text { for } n \text { and } n^{\prime} \text { odd }\end{cases}
$$

Neglecting the terms that correspond to the last case, we find:

$$
E^{2}(t) \propto \frac{1}{T} \sum_{\substack{n, n^{\prime} \\\left(n+n^{\prime} \text { even }\right)}} e^{i\left(n+n^{\prime}\right) \omega_{r} t}+\sum_{\substack{n, n^{\prime} \\\left(n+n^{\prime} \text { odd }\right)}} e^{i\left(n+n^{\prime}\right) \omega_{r} t}=\frac{1}{T} \frac{N}{2} \sum_{n} e^{i 2 n \omega_{r} t}+\frac{N}{2} \sum_{n} e^{i(2 n+1) \omega_{r} t}
$$

The first term represents the frequency doubled resonant modes and the second term are the unwanted off-resonant modes. It is seen that the power of the unwanted modes are suppressed by a factor $T^{2}$ after the first doubling stage. Note that using the suppression ratio of the fundamental light and squaring it to take the second harmonic generation into account yields the wrong result.

The action of the second doubling stage can be treated in a similar way. It is designed with the same input coupler transmission of $T=30 \%$. Again, we assume that the even modes are enhanced by $1 / \sqrt{T}$ while the odd modes are further suppressed 
by $\sqrt{T}$ :

$$
\begin{aligned}
E^{4}(t) & \propto\left(\frac{1}{T^{3 / 2}} \sum_{n} e^{i 2 n \omega_{r} t}+\sqrt{T} \sum_{n} e^{i(2 n+1) \omega_{r} t}\right)^{2} \\
& =\frac{1}{T^{3}} \sum_{n, n^{\prime}} e^{i 2\left(n+n^{\prime}\right) \omega_{r} t}+\underbrace{T \sum_{n, n^{\prime}} e^{i\left(2\left(n+n^{\prime}\right)+2\right) \omega_{r} t}}_{\approx 0}+\frac{2}{T} \sum_{n, n^{\prime}} e^{i\left(2\left(n+n^{\prime}\right)+1\right) \omega_{r} t} .
\end{aligned}
$$

The first two terms are obviously the desired even modes while the last one represents the unwanted odd modes at the fourth harmonic of the laser. We may neglect the terms proportional to $T$ relative to the terms proportional to $1 / T$. As a result, the power of the unwanted modes of the frequency comb at $205 \mathrm{~nm}$ are suppressed by $T^{4}=0.0081$ relative to the desired modes with twice the mode spacing of the laser-generated comb at $820 \mathrm{~nm}$. This estimated suppression ratio is important to determine the associated systematic shifts that are discussed in section 5.9. 


\subsection{Vacuum system and enhancement cavity}

The $205 \mathrm{~nm}$ UV light is coupled to a linear enhancement cavity with a free spectral range of $157.6 \mathrm{MHz}$ that is sketched in Fig. 3.1 and in more detail in Fig. 3.5 and Fig. 3.6. Both mirrors of this cavity have a manufacturer specified radius of curvature (ROC) of $500 \mathrm{~mm}$ with a specified uncertainty of $5 \%$. With the very well defined cavity length of $d=c / 4 f_{\text {rep }}=0.9511 \mathrm{~m}$, we calculate the focus radius to be $w_{0}=\sqrt{\lambda /(2 \pi) \sqrt{d(2 r-d)}}=80 \mu \mathrm{m}$. Since the cavity is operated close to the instability edge, this radius determines the focus size and, as a result, the AC-Stark shift. Therefore, we verified the focus size by measuring the beam radius in the farfield behind the back reflecting mirror, taking into account its defocussing action. In addition, we determined the number of transverse modes per free spectral range to be 6.9 , which again confirms the $80 \mu \mathrm{m}$ waist radius.

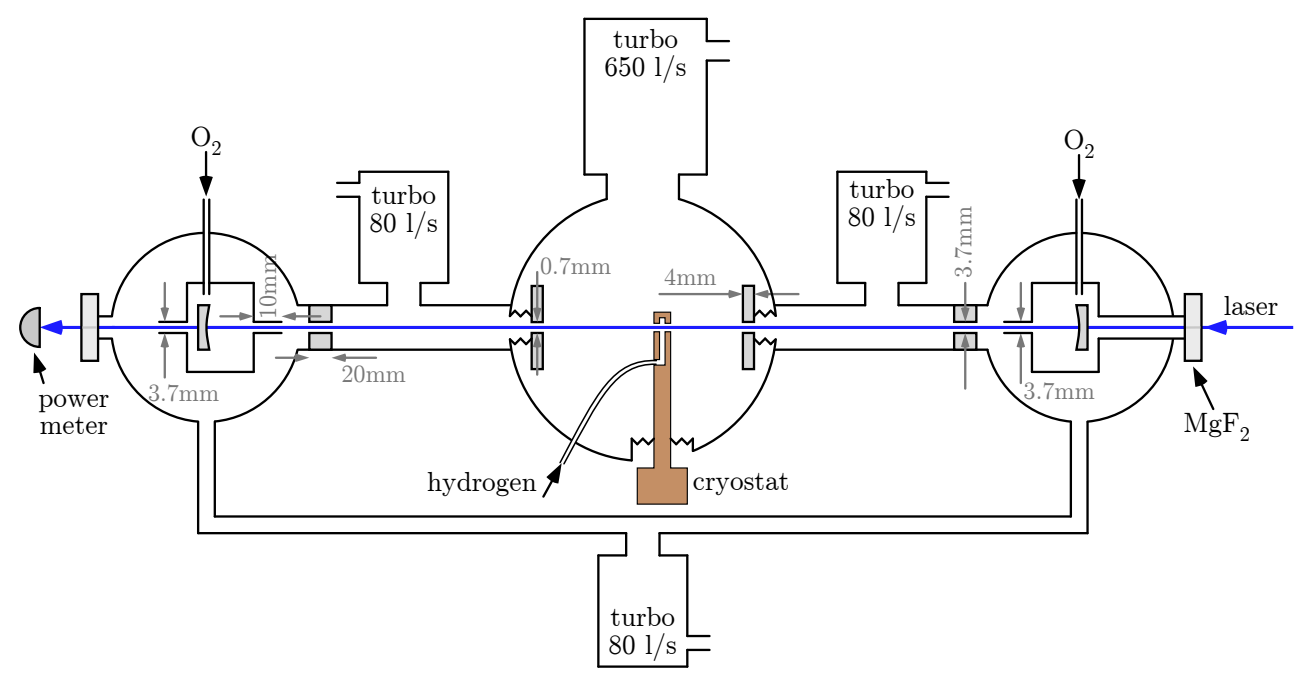

Figure 3.5.: The vacuum system consists of three chambers that are differentially pumped. The mirrors are purged with 1 mbar of oxygen to avoid UV degradation. A set of irises and pumps are arranged to maintain a pressure of $1 \times 10^{-6}$ mbar in the central chamber where the excitation takes place, which rises to around $1 \times 10^{-5}$ mbar with the hydrogen discharge on. The small $0.7 \mathrm{~mm}$ irises are mounted on transverse translation stages and sealed with flexible nitrile tubes to allow for alignment. A gas supply interlock system prevents dangerous levels of oxygen/hydrogen mixtures.

The cavity is locked with a dither-lock technique. For this, a mirror is mounted on a piezoelectric transducer and modulated between $60 \mathrm{kHz}$ and $90 \mathrm{kHz}$. The resulting amplitude modulation is detected on the cavity transmission and demodulated with a TTL signal to generate an error signal for feedback control to the cavity length.

Unfortunately, only moderate quality mirror coatings are available at a wavelength of $205 \mathrm{~nm}$ with losses of $2-3 \%$. The input coupler has a transmission of $T_{1}=6.4 \%$ 
and a reflectivity of around $R_{1}=90 \%$ while the back mirror has a reflectivity of $R_{2}=97 \%$ with a very small transmission (Laseroptik $\mathrm{GmbH}$ ). These values are reached at wavelengths between $201 \mathrm{~nm}$ and $210 \mathrm{~nm}$ and hence with a bandwidth much larger than the bandwidth of the ps-comb (see Fig. 3.3). For the observed loss, this choice for the input coupler provides the best coupling. Nominally, the power reflection is only $0.3 \%$ on resonance. The power enhancement is given by

$$
U=\frac{T_{1}}{\left(1-\sqrt{R_{1} R_{2}}\right)^{2}+4 \sqrt{R_{1} R_{2}} \sin ^{2}(\theta)}
$$

with $\theta \equiv 2 \pi d / \lambda$. On resonance $(\theta=0)$, the laser power that is circulating inside the cavity (per propagation direction) is expected to be 15 fold enhanced relative to the impinging laser power. However, these considerations assume perfect spatial mode matching. To improve the laser power calibration we determined the transmission of the output coupler to be $T_{2}=5.4 \times 10^{-4}$ by measuring the power levels before and after this mirror without the input coupler in place. By using the actual laser spectrum, small spectral variations of the transmissivity are properly accounted for. The power enhancement determined in this way is only 10 , which means that around $66 \%$ of the impinging laser power is spatially mode matched to the cavity. This value seemed to be limited by significant deviations from a $\mathrm{TEM}_{00}$ mode exiting the second doubling stage due to walk-off within the crystal. This value roughly agrees with the observed reflected power drop when when the cavity is scanned over the resonance. The calibration of the output mirror transmission is not only important to verify the power enhancement, but also to verify the expected AC-Stark shift (see section 5.2).

As has been observed in many previous experiments, mirror reflectivities can quickly degrade when exposed to intense light in vacuum - particularly at short wavelengths. Presumably residual organic compounds are dissociated at high intensities and form a carbon layer on the mirror surfaces [53]. Mostly these degradations are reversible in the presence of oxygen. We observe an almost complete degradation of the cavity finesse within a few minutes, but can fully prevent it with oxygen. Therefore, it seems essential that the mirrors are well-separated from the cavity focus. This, and the rather lossy mirror coatings, are the reason for our linear cavity design that unfortunately needs to be operated not too far from the stability edge. A differentially pumped vacuum system has been designed for this purpose. It allows us to maintain a low pressure of $1 \times 10^{-6}$ mbar inside the center of the chamber where hydrogen excitation takes place while surrounding the mirrors with about 1 mbar of $\mathrm{O}_{2}$. To achieve this, the mirrors are enclosed in boxes with holes for the laser beam. The flow of oxygen out of these holes flushes the inside of the vacuum windows where the light intensity is lower. We use four vacuum pumps to maintain these pressure gradients as shown in Fig. 3.5 and Fig. 3.6.

To stabilize the $205 \mathrm{~nm}$ enhancement cavity to the frequency comb, its output mirror is mounted on a ring shaped piezo transducer that is modulated with a frequency between 60 and $90 \mathrm{kHz}$ and an amplitude of $<0.1 \mathrm{~nm}$ (see section 5.7). The 


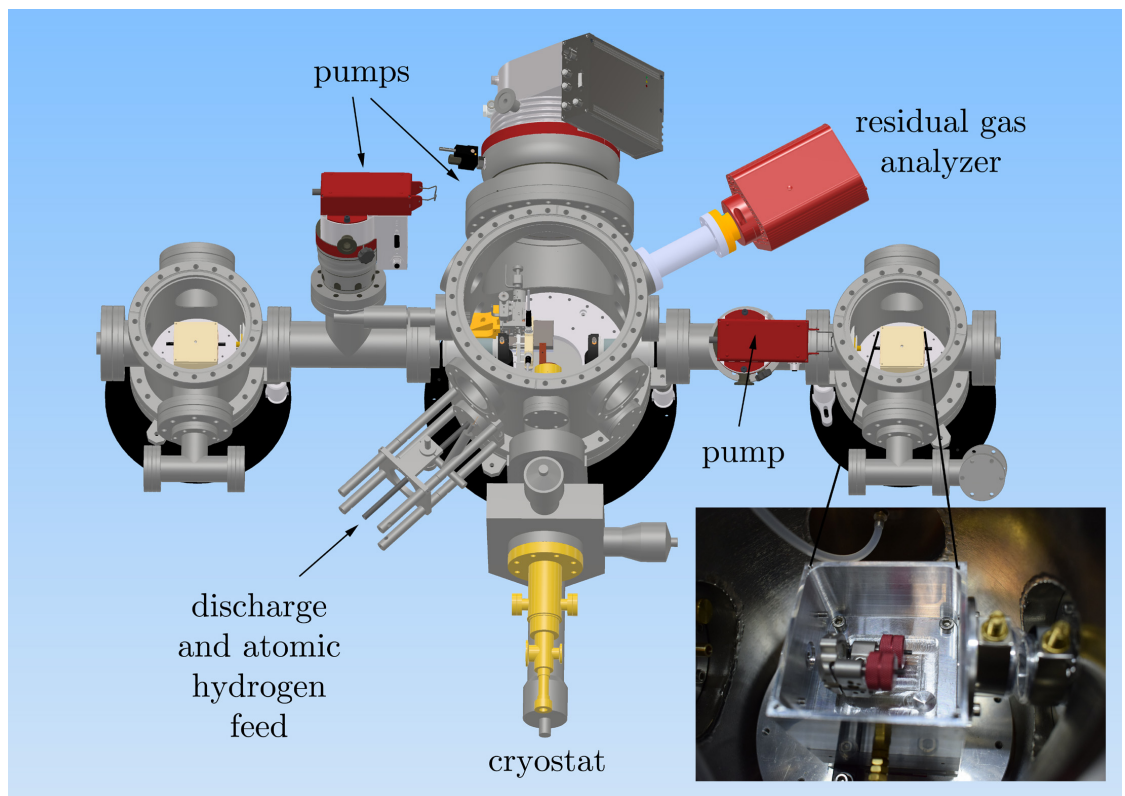

Figure 3.6.: CAD drawing of our vacuum system with differential pumping stages as sketched in Fig. 3.5. The 1S-3S excitation takes place in the central chamber within the pulse collision volume (see Fig. 3.1, 3.9). Also shown are the liquid helium (LHe) cryostat that holds the nozzle as well as the gas discharge to generate atomic hydrogen. Photographs of the nozzle and the discharge setup are shown in Fig. 3.7. The cavity mirrors are enclosed in oxygen purged boxes; one of them is shown in the photograph inset at the lower right corner.

cavity transmission is detected with a UV photo detector (not shown in the figures) and is demodulated with a lock-in amplifier to generate an error signal. This error signal is sent to a loop filter and used to actuate the same piezo transducer at lower frequencies. Systematic shifts and possible line shape distortions due to this modulation are discussed in section 5.7.

The UV photo detector receives only $10 \%$ of the transmitted power while a larger fraction (10's of $\mu \mathrm{W}$ ) is sent to a Si photodiode-based laser powermeter (Thorlabs, S120VC) for AC-Stark shift characterization (see section 5.2). For this purpose we use a beam splitter at almost $90^{\circ}$ incidence to suppress the polarization sensitivity of the measurement.

In general, the enhancement cavity can introduce a chirp to the pulses even when the dispersion of the mirror coating is neglected (well justified for ps pulses). Since the only parameter that is used to control the enhancement resonator is its length, not all modes are resonant simultaneously. The modes of the incident frequency comb that are not exactly resonant are enhanced with a spectral phase given by $\tan (\varphi)=\sqrt{R_{1} R_{2}} \sin (2 \theta) /\left(1-\sqrt{R_{1} R_{2}} \cos (2 \theta)\right)$. To estimate this effect, we assume that one mode of the comb is exactly locked to a cavity mode while the mode spacing mismatch between the cavity and the comb leads to a spectral phase. We 
further assume that the free running carrier envelope offset frequency of the comb is less than the repetition rate. The maximum offset within its spectral width of $\Delta \omega=2 \pi \times 148 \mathrm{GHz}$ is reduced by the relative optical bandwidth of $1.0 \times 10^{-4}$, i.e. $\theta_{\max }=1.0 \times 10^{-4} \pi$ and $|\varphi|=8.9 \mathrm{mrad}$. While a linear spectral phase does not chirp the pulses, we assume that the cavity imposed spectral phase is purely quadratic. This leads to a pulse broadening of $\sqrt{1+\left(2 \theta_{\max } / \tau^{2} \Delta \omega\right)^{2}}=1.000013$ and a corresponding chirp parameter of $b=0.0051$ that can be safely ignored. 


\subsection{Atomic hydrogen beam}

To form a cryogenic atomic hydrogen beam [54], we first dissociate molecular hydrogen in a gas discharge. A $6 \mathrm{~mm}$ diameter Pyrex tube is surrounded by a $2.4 \mathrm{GHz}$ radio frequency cavity with $30 \mathrm{~W}$ of input power. The pressure inside the tube is around $8.6 \mathrm{mbar}$ and the total hydrogen flux is very constant at around $0.37 \mathrm{ml} / \mathrm{min}(1.7 \times$ $10^{17}$ molecules/s) and continually monitored with a mass flow meter (Bronkhorst, EL-Flow). At the outlet of the tube there is a small orifice $(300-500 \mu \mathrm{m}$ diameter). Behind the orifice a $30 \mathrm{~cm}$ long Teflon tube with $4 \mathrm{~mm}$ inner diameter $(6 \mathrm{~mm}$ outer diameter) guides the dissociated hydrogen into the copper nozzle. The nozzle consists of a box-shaped reservoir (inner dimensions $7.8 \times 7.0 \times 9.4 \mathrm{~mm}^{3}$ ) with two orifices through which hydrogen escapes into vacuum and which allow the laser beam to pass through the nozzle. The orifice that faces the pulse collision volume $(\mathrm{PCV})$ has a diameter of $1.5 \mathrm{~mm}$ and the rear orifice a diameter of $1.3 \mathrm{~mm}$. We use a liquid helium flow cryostat (ICE Oxford) to cool the nozzle (minimal temperature $4 \mathrm{~K})$. A short part of the Teflon tube $(\sim 8 \mathrm{~mm})$ is clamped to the nozzle for mounting and is assumed to be at a temperature close to that of the nozzle. The temperature is measured both inside the copper cooling head of the cryostat (sensor: LakeShore, CX-1030-AA-1.4L) and on top of the hydrogen nozzle above the thermalization volume (sensor: Lake Shore DT-670B-SD). The temperature is stabilized to within $10 \mathrm{mK}$ through a feedback loop (heating wire inside the cooling head). Good temperature stability is not only important for characterizing the second-order Doppler effect (see section 5.3), but also to reduce excess noise because the flow of atomic hydrogen critically depends on the nozzle temperature. Figure 3.7 shows photographs of the discharge tube and the nozzle.

The hydrogen atoms thermalize to the nozzle's temperature through collisions with the cold walls of the nozzle's reservoir and the cold section of the Teflon tube, or collisions with other atoms or molecules, with at least one collision necessary for the atoms to leave the nozzle. Using a particle tracing simulation, the number of collisions the atoms undergo with these cold walls before leaving the nozzle is estimated. Collisions between the particles are not included in the simulation, but are thought to increase the total number of collisions above the number of wall collisions. The mean number of wall collisions is found to be 60 , with most of the atoms undergoing 15 collisions. In total, $99.6 \%$ of the atoms undergo more than one wall collision. We expect this number of collisions to be sufficient for good thermalization based on the observations of $[54,62]$ where nozzle designs with a similar number of collisions were studied. We note that, due to the nozzle's geometry, atoms traveling on-axis with the laser beam can only originate from collisions with other atoms or molecules and not from the cold walls. In fact, collisions between these particles should allow for faster thermalization than wall collisions, since the mass ratios of the collision partners are well matched. However, these collisions also tend to remove slower atoms from the beam, as discussed in section 5.3.

In first order a thermal beam of atoms may be described with a Maxwellian 

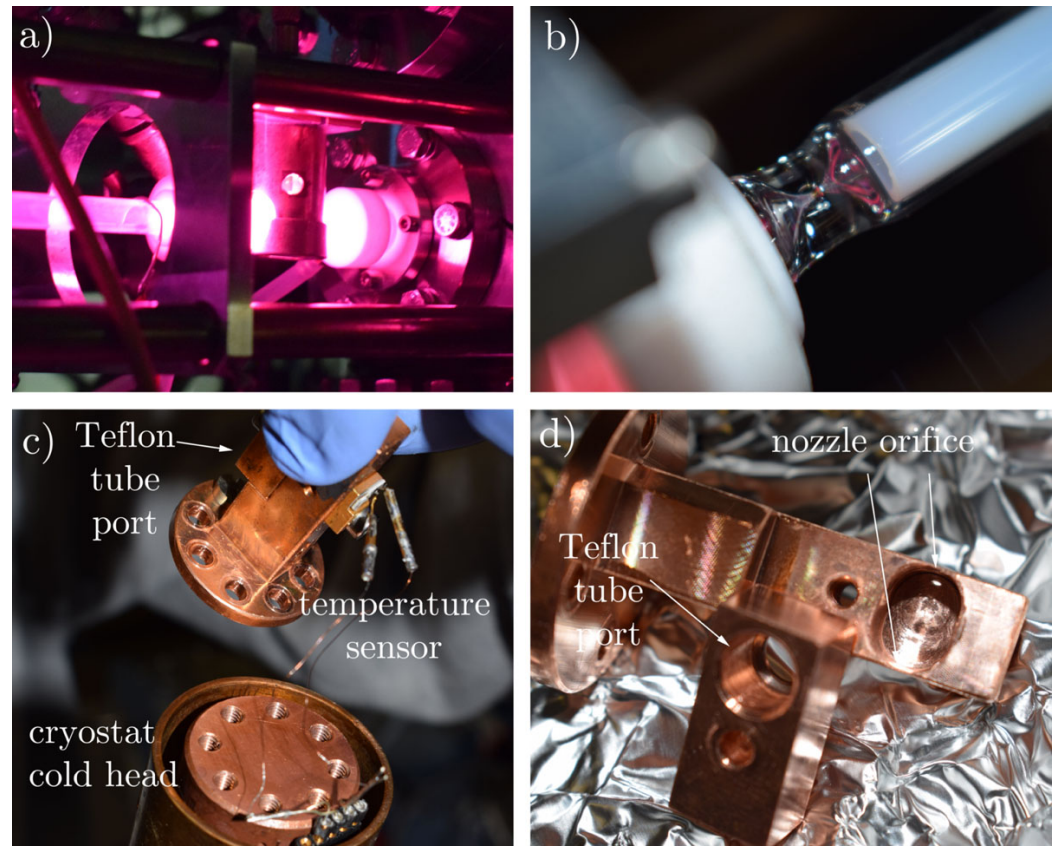

Figure 3.7.: a) The radio frequency discharge in operation with Balmer emission visible. b) Pyrex discharge tube with the small smooth orifice visible along with the Teflon tube guiding the atomic hydrogen to the cryogenically cooled nozzle. c) Hydrogen nozzle together with a temperature sensor and the cooling head of the cryostat. d) Copper nozzle with Teflon tube connector. Hydrogen enters through the Teflon tube into the thermalization volume and exits into vacuum through two orifices parallel to the laser beam.

velocity distribution of $p(v) \sim v^{2} e^{-\left(v / v_{0}\right)^{2}}$ with the most probable velocity $v_{0}=$ $\sqrt{2 k_{B} T / m}$ and the atomic mass $m$. The number of contributing atoms per second is then given by the flux which gives an additional factor $v$ in the distribution [61]. To estimate the expected signal, the velocity-dependent excitation probability needs to be taken into account. Slower atoms, i.e. at low temperatures, reach a steady state while travelling through the PCV, so that their signal is proportional to the travel time, i.e. $\sim 1 / v$ (see also section 5.3). Therefore the main contribution to the signal is due to the most probable velocity $v_{0}$, which is $340 \mathrm{~m} / \mathrm{s}$ at a temperature of $T=7 \mathrm{~K}$.

To estimate the flux of hydrogen atoms from the measured input flux of hydrogen molecules, we need to determine the degree of dissociation $\alpha=\mathrm{N}(\mathrm{H}) /(\mathrm{N}(\mathrm{H})+$ $2 \mathrm{~N}\left(\mathrm{H}_{2}\right)$ ). Unfortunately, this depends on many parameters such as the design of the gas discharge (radio frequency power, material of the tube, the tube surface quality and temperature, the orifice shape and diameter etc.) as well as on the hydrogen recombination losses inside the Telfon tube and the nozzle. The latter depends on both the temperature of the surfaces and the particle densities [54]. One way to estimate the degree of dissociation is to observe the background gas composition with a residual gas analyzer with and without the discharge but with constant molecular 


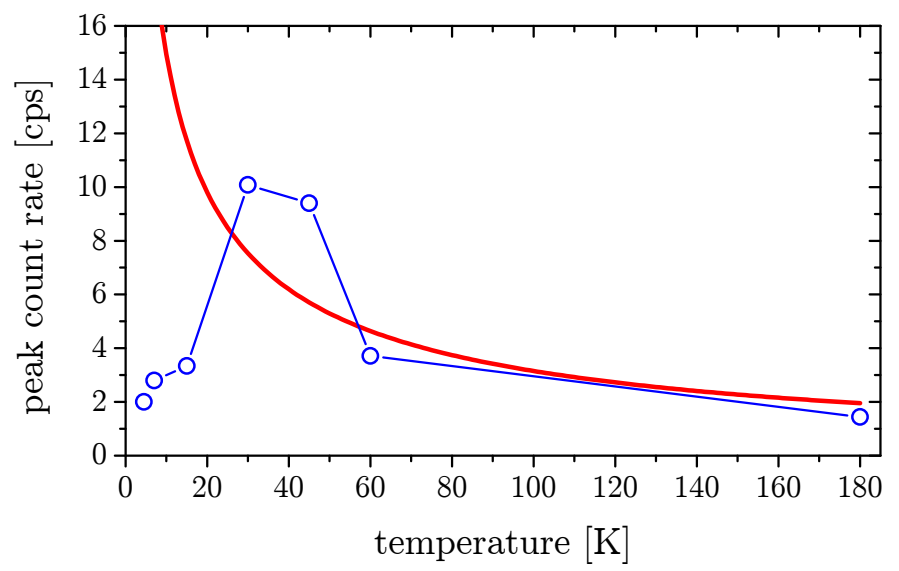

Figure 3.8.: Comparison of the experimentally measured Doppler-free count rate (blue) with a Monte Carlo simulation (red) (see section 2.5) that ignores recombination. We do not know the scaling of the simulation data and plot it here such that it roughly agrees with the experimental count rates at higher temperatures. The main purpose of the figure is to show that with the current configuration, it is not possible to go to significantly lower temperatures without a prohibitively large reduction of the signal count rate. From the comparison of the experiment with the simulation the temperature dependence of the degree of dissociation $\alpha$ can be estimated. The experimental data and simulations in this plot are for a pulse collision volume distance to the nozzle of $d=27.1 \mathrm{~mm}$.

input flux. We have found that the $\mathrm{H}^{+}$signal from our residual gas analyzer (Pfeiffer Vacuum) is not a good metric since these ions are also produced from many organic compounds at the ionizer stage of the analyzer's input. In addition, the analyzer is not put directly in the path of the hydrogen beam so that recombination of hydrogen atoms can occur before detection. Nevertheless, we observe a reduction of the $\mathrm{H}_{2}^{+}$ signal of $5 \%$ to $10 \%$ when the discharge is turned on, which we use as an estimate for $\alpha$.

Another way to estimate the degree of dissociation is to compare the experimental count rates on resonance at different temperatures to an ab initio Monte Carlo simulation described in more detail in section 2.5. This model solves the optical Bloch equations for a number of thermal atomic trajectories. The overall quantum efficiency of the detection scheme (see next section) is modeled by ray tracing. By comparing the model (which assumes $\alpha=100 \%$ ) to the experimental data, we can estimate the temperature dependence of the true degree of dissociation. Roughly speaking, the predicted count rate is expected to drop as $\propto 1 / v_{0} \propto 1 / \sqrt{T}$ with higher temperatures $T$ due to time-of-flight effects (see above). This means that if the flux of atoms would stay constant for all temperatures, the count rate would increase with decreasing temperatures. As shown in Fig. 3.8, simulations predict an increase of the count rate by a factor of 9.4 from $180 \mathrm{~K}$ to $7 \mathrm{~K}$, whereas experimentally an increase of only 1.9 is observed. This behavior is consistent with a strong increase in the hydrogen recombination coefficients for both copper and Teflon surfaces below 
$60 \mathrm{~K}[55]$. We do not expect a solid $\mathrm{H}_{2}$ layer to form on the cold surfaces at any temperature except at $4.5 \mathrm{~K}$, where its formation was observed in the experiment. This is because even at $7 \mathrm{~K}$, the vapor pressure of solid $\mathrm{H}_{2}\left(2.2 \times 10^{-2} \mathrm{mbar}\right)$ is much larger than the pressure inside the nozzle as determined through a simulation $\left(8 \times 10^{-5} \mathrm{mbar}\right)$. The presence of such a layer is expected to reduce recombination substantially $[54,55]$. However, in the experiment, the count rate at $4.5 \mathrm{~K}$ is well below its value at $7 \mathrm{~K}$. We attribute this to the presence of a thermal gradient along the Teflon tube, which is held at nozzle temperature at one end and at room temperature at the other end. Thus, at some point along the tube, the temperature will be too high for solid $\mathrm{H}_{2}$ to form, but low enough for large recombination to occur.

\subsection{Fluorescence detection}

For a well adjusted delay line (see fig. 3.1), the two counter propagating pulses circulating in the $205 \mathrm{~nm}$ enhancement cavity collide at its centre in the pulse collision volume (PCV). The Doppler-free signal emerges only from near the PCV because atoms at the most probable thermal velocity of $320 \mathrm{~m} / \mathrm{s}$ travel only $54 \mu \mathrm{m}$ during the $158.37 \mathrm{~ns}$ lifetime of the excited state. Upon decay to the $2 \mathrm{P}$ state, Balmer- $\alpha$ light at $656 \mathrm{~nm}$ is emitted, followed by Lyman- $\alpha$ at $121 \mathrm{~nm}$ for the decay to the ground state. We collect only the light from the first decay, which is isotropically emitted in $4 \pi$, with four aspheric lenses (Thorlabs, C330TMD-B, focal length $3.1 \mathrm{~mm}, \mathrm{NA}=0.68$ ) and image it onto the entrance facets of multimode fibres. The light is guided out of the vacuum chamber using fibre feed-through flanges. At the fiber outputs, we recollimate the light, filter with bandpass interference filters (bandwidth $10 \mathrm{~nm}$ ), and focus onto photon counting modules (Hamamatsu, H7421). Two of the four lenses image the fluorescence light onto $1 \mathrm{~mm}$ core diameter multimode fibres and their optical axis goes through the centre of the PCV. The light from these two fibres is detected with the same photon counting module (the $j=1$ detector). The two auxillary fibres are shifted by about $\pm 0.6 \mathrm{~mm}$ along the laser propagation direction ( $z$-axis) and image the tips of the cigar-shaped PCV onto $0.6 \mathrm{~mm}$ multimode fibres, each guiding the light to separate photon counting modules (the $j=2$ and $j=3$ detectors). In this way we obtain three Doppler-free (DF) signals, each imaging a different portion of the PCV. Figure 3.9 shows the arrangement.

As described in more detail in section 2.5, we use a Monte Carlo ray tracing method to determine the light collection profiles as a function of the radial distance from the optical axis defined by the lens. This profile is shown for the large and small fibres at the left hand side of fig. 3.10. The right hand side of this figure shows the expected and measured signal strength received from the PCV as a function of the lens position along the atomic beam.

We cannot exclude small chirps of our $205 \mathrm{~nm}$ laser pulse train which are known to gives rise to a "chirp induced residual first-order Doppler shift" (CIFODS) when 


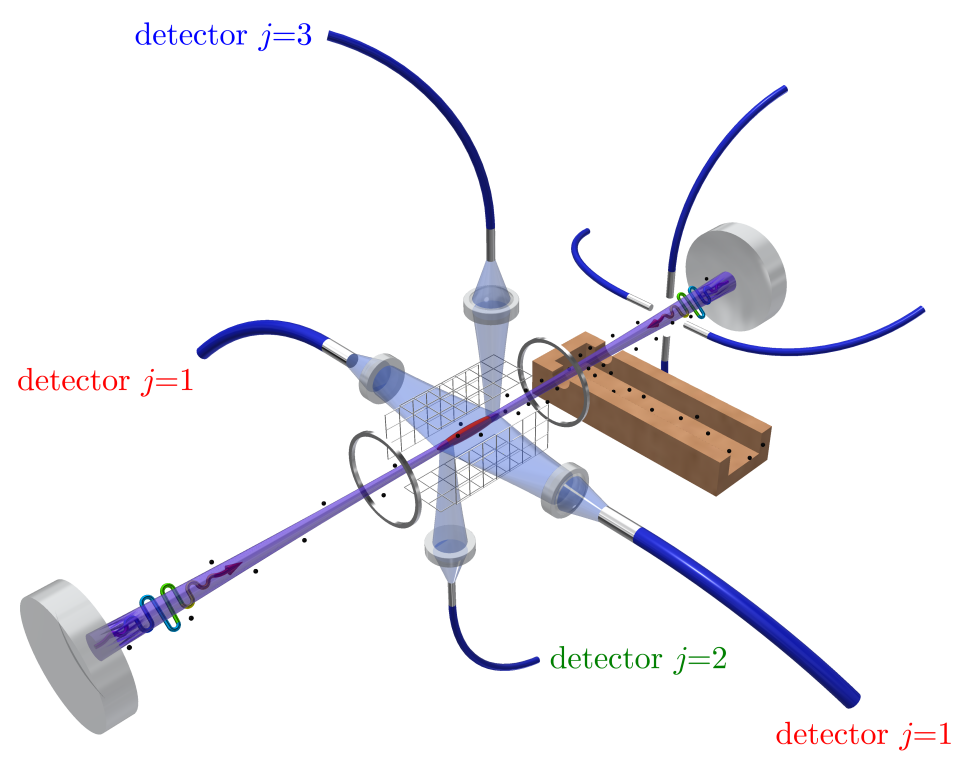

Figure 3.9.: The pulse collision volume (PCV) that is shown in red (also in fig.3.1), resembles an ellipsoid with semi-axis $w_{0}=80 \mu \mathrm{m}$ and $c T_{1 / 2}=600 \mu \mathrm{m}$. It is surrounded by a Faraday cage made of a highly transmissive mesh and two ring electrode. Employing the quadratic DC-Stark shift we can put tight limits on stray electric fields by applying voltages to the cage in all three directions and determining the minima of the resulting line shifts (see supplemental material). Four lenses image the fluorescence from the whole PCV and its ends to multimode fibres $(1 \mathrm{~mm}$ and $600 \mu \mathrm{m}$ diameter $)$ that guide the light through interference filters onto three independent single-photon counting modules; one main $j=1$ and two auxiliary $j=2,3$. With this arrangement we can interpolate the chirp induced residual first order Doppler effect (CIFODS). At the other side of the nozzle, the Doppler broadened signal is collected with four bare fibres of $1 \mathrm{~mm}$ core diameter that are in close proximity to the laser and atomic beam. As the Doppler broadening is well in excess of the mode spacing, this signal is independent of the laser frequency and used for normalization. This effectively removes significant fluctuations of the laser power and the atom number flux. Since the Doppler free and the Doppler broadened signal scale in the same way with laser power, the normalized line amplitudes can be used as a measure of the atom number flux.

used with a diverging atomic beam [21]. This effect is discussed in detail in section 5.1. The main detector $(j=1)$ is the least sensitive to CIFODS, while the two auxiliary detectors have strong dependence of opposite signs. This combination is a powerful tool to compensate the CIFODS as described in section 4.4.

Also shown in fig. 3.9 are four additional $1 \mathrm{~mm}$ multimode fibres that collect light directly from the atomic beam without lenses. All four of them feed a single detector. They are located on the other side of the nozzle and therefore only receive the Doppler-broadened (DB) fluorescence (two photons one direction). The $\sigma_{+}$and $\sigma_{-}$polarisation transfers two units of angular momentum so that the 1S-3S transition is forbidden. However, all hyperfine components of the 1S-3D transitions are allowed, 

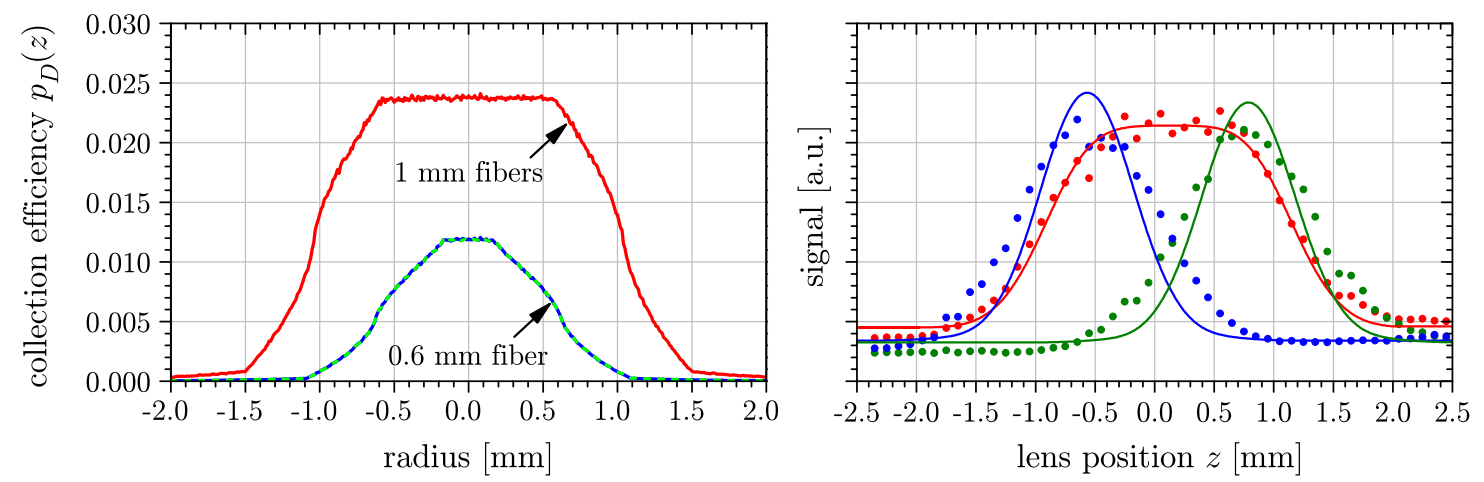

Figure 3.10.: Left: Light collection profiles of the lenses used to couple the Balmer- $\alpha$ fluorescence of the atoms into multimode fibers with core diameters of $600 \mu \mathrm{m}$ (green/blue, $j=2$ and $j=3$ detector) and $1000 \mu \mathrm{m}$ (red, $j=1$ detector). A Monte Carlo ray tracing method was used to determine the fraction of light that ends up in the fiber core when emitted from a point at a given radial distance from the optical axis (perpendicular to the atomic beam). The depth of focus is neglected. While the large fiber essentially collects the light from the full pulse collision volume, both fibers cover the radius of the pulse collision volume of $w_{0}=80 \mu \mathrm{m}$. Therefore, we apply these collection profiles only along the $z$ axis, i.e. along the atomic/laser beam axis. The red curve shows the expected collection efficiency for both fibres that feed the $j=1$ detector. The green/blue curves are for one of the $j=2$ and $j=3$ detectors, so that their maximum appears smaller by a factor of two. For the overall detection efficiency, the $24 \%$ quantum efficiency of the photo detector has to be included. Hence the total quantum efficiency for the main detector is estimated to be $0.6 \%$. Right: The solid lines show the collection profiles folded with the emission from the pulse collision volume (see section 2.5) as a function of the lens position along the atomic beam (z-axis). The data points are the corresponding measurements that have been scaled and shifted by a common offset to match the simulations.

except for the $1 \mathrm{~S}(F=0)-3 \mathrm{D}_{3 / 2}(F=1)$ and the $1 \mathrm{~S}(F=0)-3 \mathrm{D}_{5 / 2}(F=0)[42]$. The 3S-3D fine structure spreads over $4013 \mathrm{MHz}$ and hence lies within the bandwidth of our $205 \mathrm{~nm}$ frequency comb (see fig. 3.3). The Doppler width is on the order of $4.5 \mathrm{GHz}$, so that the modes of the comb are not resolved. This makes the signal independent of the laser detuning, which is bad for spectroscopy but perfect for normalizing for fluctuations of the laser power and atomic flux (see section 4.2). In fact this is one of the advantages of the two-photon direct comb spectroscopy in an atomic beam. We have mounted the DB detector fibres together with the nozzle on the same translation stage. In this way we obtain the proper relative normalization when shifting the nozzle relative to the PCV for measuring the pressure shift (see section 5.4 for details). Since the DB signal emerges from a multiple of excitation and decay paths it is not trivial to determine it spatial emission pattern. Using the full set of optical Bloch equations as described in section 2.5, we find that $91 \%$ of the light is emitted in one of the $\sigma$-components with the $z$-axis along the laser beam. The chosen polarization and $z$-axis do not occupy coherent superpositions of 


\begin{tabular}{||l|r|r||}
\hline Contribution & DF detector & DB detector \\
\hline \hline Doppler-free & 2000 & 0 \\
\hline Doppler-broadened & 500 & 50000 \\
\hline Recombination & 30 & 1000 \\
\hline Dark counts & 50 & 50 \\
\hline Laser scatter & 20 & 2000 \\
\hline \hline Total & 2600 & 53000 \\
\hline
\end{tabular}

Table 3.1.: Typical contributions to the total count rates $(\mathrm{Hz})$ of the main Doppler-free (DF) detector (similar for the auxiliary DF detectors) and the Doppler-broadened (DB) detector. In addition to the DF and the DB counts, we also detect dark counts, scattered laser photons, and recombination counts. Note that we refer to the detectors that record light from the pulse collision volume as DF, even though they also register a Dopplerbroadened background.

the same Zeeman multiplets. Hence, ignoring small quantum inference effects (see section 5.8) we conclude that the emission pattern is rotational symmetric about the $z$-axis.

Besides DF and DB fluorescence we detect some scattered laser light at $205 \mathrm{~nm}$ that enters the fibres, is absorbed, and down converted. A fraction of the down converted light happens to occur within the bandwidth of the bandpass interference filters. Further, hydrogen atoms can recombine to form molecules on surfaces or hydrogen molecules can show chesorluminescence [58]. We have observed rather strong emission originating from processes on aluminum surfaces. Some of it also passes the bandpass interference filters and gives rise to a background that we refer to as recombination counts. Table 3.1 summarizes the different contributions to the DF and DB photon count rates. Note, that while the DF detectors register both Doppler-free and Doppler-broadened counts, the DB detector does not register any Doppler-free counts. Further the total count rate of the DB detector is roughly twenty times larger than the count rate of the DF detector. This is because the DB detector can be placed very close to the hydrogen nozzle and the fibres can be put very close to the laser beam, thus covering a large solid angle (at the cost of larger recombination and scatter count rates). From table 3.1 the contrast (peak count rate divided by the background count rate) is approximately $C \approx 4.3$. To calculate the theoretical contrast using the Monte Carlo simulation, we need to take into account the correct polarization $\sigma_{ \pm}$, all resonant fine- and hyperfine levels (cf. fig. 3.2) as well as the difference in the detection profile for Doppler-free and Doppler-broadened counts. The latter are emitted with the same strength within the entire atomic beam and are only limited by the geometrical properties of the detector (cf. fig. 3.10, left). On the contrary, Doppler-free counts are only emitted within the PCV and the geometrical detection profile of the fibre-lens detector has to be convoluted with the intensity profile of the PCV. Taking all this into account, we obtain a theoretical 
contrast of approximately $C \approx 6.55$, which is slightly better than the experimentally observed contrast. 



\section{Data Evaluation}

\subsection{Data Description}

The measurement campaign was carried out in two sessions in March and April of 2018 with a total of 11 measurement days. The measurement data and description files can be found in [50]. Different key parameters such as the nozzle temperature, its distance to the PCV, the laser power, and the chirp of the laser pulses were varied in order to experimentally characterize systematics such as the second-order Doppler shift, pressure shift, AC-Stark shift and residual first-order Doppler shifts (CIFODS). Overall 4450 valid line scans have been recorded, most of which contain 21 data points of $1 \mathrm{~s}$ gate time with a total duration of $36 \mathrm{~s}$ (see Fig. 4.1). To improve the statistics we do not distribute the data points uniformly on the frequency axis but so that they appear roughly equidistant on the signal axis (see lower right of Fig. 4.2). For this we employed the following line sampling grid:

$$
\begin{array}{llrr}
-4.000000 \mathrm{MHz} & -1.796820 \mathrm{MHz} & -1.271250 \mathrm{MHz} & -0.992032 \mathrm{MHz} \\
-0.804280 \mathrm{MHz} & -0.661160 \mathrm{MHz} & -0.542312 \mathrm{MHz} & -0.436248 \mathrm{MHz} \\
-0.334024 \mathrm{MHz} & -0.223112 \mathrm{MHz} & 0.000000 \mathrm{MHz} &
\end{array}
$$

with the mirrored positive frequencies. In this way, we put additional weight on the parts of the line shape with the largest slope - that is with the largest sensitivity to the line position. The frequency points are measured pairwise symmetric in random order but with the central point always first. Randomizing the sequence of measurement points is important. Otherwise the continuous degradation of the second doubling stage, as described in section 3.2, would lead to systematic line shifts.

Besides the cycle slip criteria described in section 3.1, we apply additional data selection rules. To remove glitches in the laser power, for example by drop-outs of the $205 \mathrm{~nm}$ cavity lock, we discard points where the DB counts deviate by more than 3 standard deviations from the line-averaged DB counts. We apply the same criteria to the recorded transmitted laser power through the cavity. In addition, we discard points that received less than $30 \%$ of the maximum DB counts of the respective line scan. We discard the full line scan if it contains less than 10 data points after these selections. In total, we removed $5.6 \%$ of data points from the line scans and removed a total of 369 full line scans. Figure 4.1 gives an overview of the number of valid data points per line scan. We verified that the variation of the thresholds within reasonable limits has no effect on our final result. 


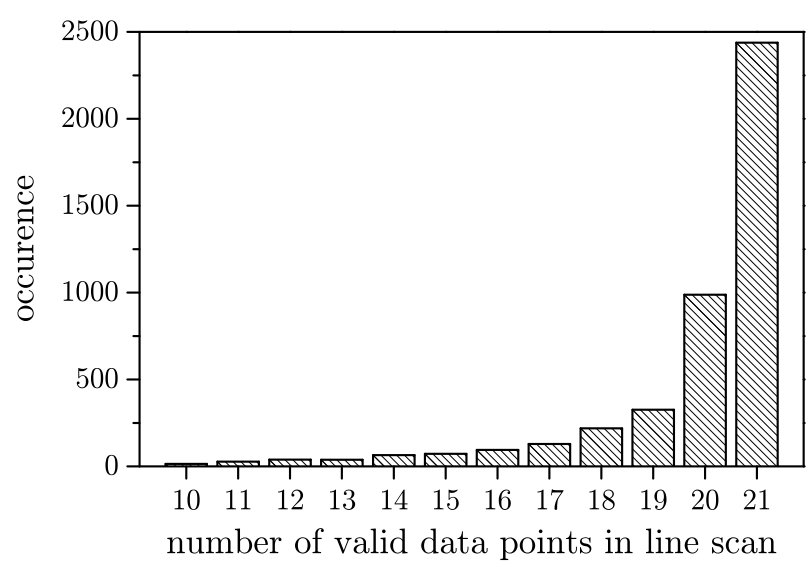

Figure 4.1.: Histogram of the number of valid data points within the 4450 line scans. There are 2438 line scans with the full 21 data points, 988 with 20 data points, 326 with 19 data points, 219 with 18 data points, and 479 with 16 and fewer data points. Line scans with less than 10 data points are discarded as a whole.

\subsection{Line fitting and normalization}

We fit the measured lines with a simple Lorentzian:

$$
\mathscr{L}\left(\nu, \nu_{0}, a, \gamma, y_{0}\right)=y_{0}+a \frac{(\gamma / 2)^{2}}{\left(\nu-\nu_{0}\right)^{2}+(\gamma / 2)^{2}} .
$$

The fit parameters are the center frequency $\nu_{0}$, the line amplitude $a$, the line width (FWHM) $\gamma$ and a constant background $y_{0}$.

As detailed in section 3.6, each data point of the Doppler-free 1S-3S signal (DF) is normalized by the simultaneously recorded Doppler-broadened (DB) 1S-3D signal. This means that the amplitudes $a$ do not depend on the laser power. They do however depend on the nozzle-PCV distance because the DB detector is translated with the nozzle while the DF detector is not. This will be important for the global minimization discussed in section 4.4. To assign error bars, we first assume pure shot noise of both of these signals and apply the usual error propagation. Figure 4.2 shows a line scan with larger than typical noise for illustration before (Fig. 4.2 a)) and after normalization (Fig. $4.2 \mathrm{~d}$ )) together with a fitted Lorentzian. To measure the quality of the fit, we use the minimized $\chi^{2} /$ dof, where dof are the degrees of freedom; i.e. the number of data points minus the number of fitted parameters. For lines without cycle slips or otherwise discarded points, we have dof $=17$. The values of $\chi^{2} /$ dof, are expected to follow a $\chi^{2}$-distribution with an expectation value 1 and standard deviation of $\sqrt{2 / \text { dof }}$, provided that all data is normal distributed. As one can see in Fig. 4.2, the normalization is very effective and reduces $\chi^{2} /$ dof from 47.96(34) to 1.85(34) for this particular line scan.

To further investigate the remaining noise (above shot noise), we first plot a histogram of the $\chi^{2} /$ dof values of all analyzed line scans in Fig. 4.3 (black line). The 

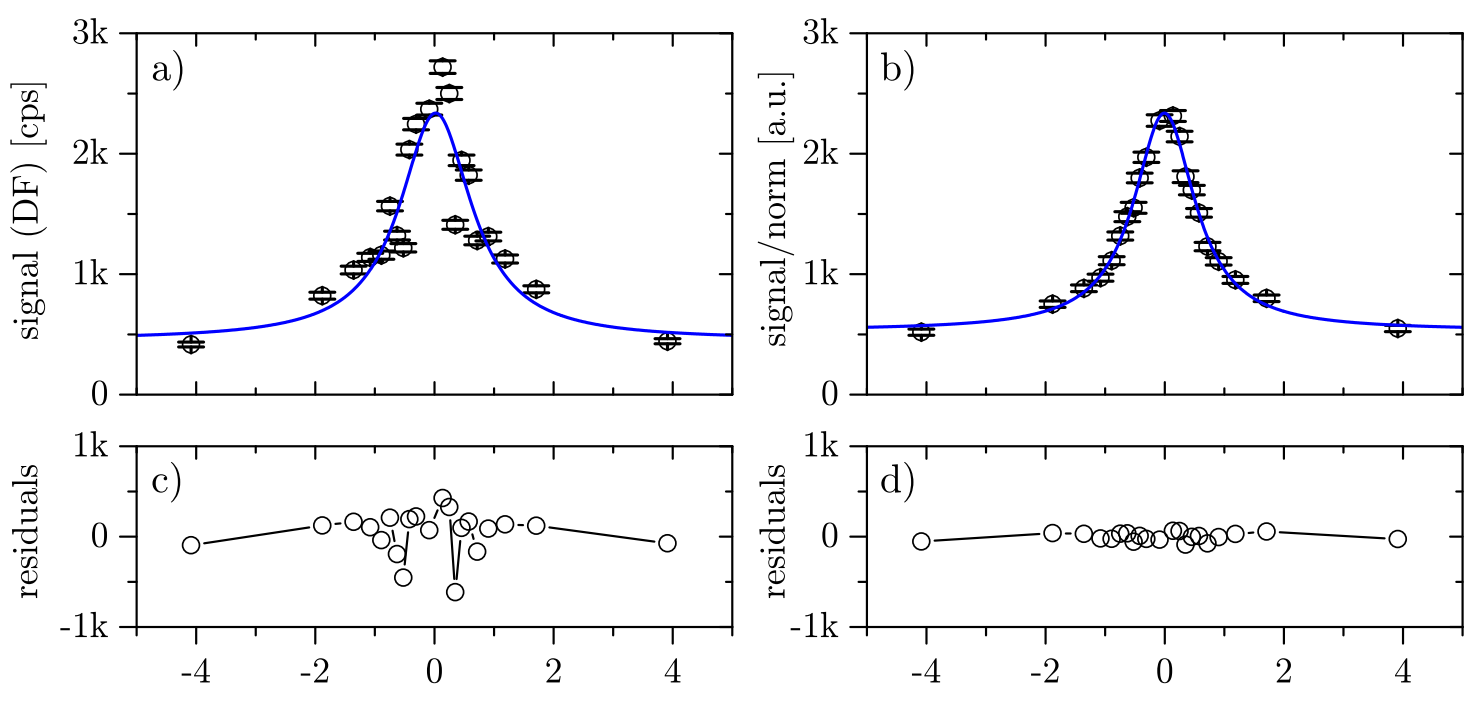

laser detuning at atomic frequency $[\mathrm{MHz}]$

Figure 4.2.: a) Doppler-free (DF) count rate before normalization together with a Lorentz fit. The noise clearly exceeds the shot noise level given by the error bars $\left(\chi^{2} /\right.$ dof $\left.=48.0\right)$. b) Normalizing by the Doppler-broadened (DB) count rate, as described in section 3.6, significantly reduces the noise and takes us close to the shot noise level given by the error bars $\left(\chi^{2} /\right.$ dof $\left.=1.85\right)$. The normalized signal has been scaled for this plot to match the nonnormalized signal in order to compare the fit residuals shown in c) and d). This particular line is not typical but has been chosen for a larger than average noise for illustration (see Fig. 3.2 for a line scan with lower noise). It was recorded at a temperature of $7 \mathrm{~K}$, with a measured laser power of $P=52 \mu \mathrm{W}$ and a nozzle PCV distance of $d=27.1 \mathrm{~mm}$ and a line width of 1.254(46) MHz.

observed mean value is shifted from the theoretical $\chi^{2} /$ dof distribution (red) and a tail of large $\chi^{2} /$ dof values is present. The main reason is the instability of the spectral envelope of the comb (see Fig. 3.3) and the usage of the 1S-3D lines to normalize the 1S-3S signal. It turns out that line scans with a large amplitude $a$ are more often fitted with a large $\chi^{2} /$ dof, presumably because the relative shot noise contribution is small, so that other noise sources dominate. To investigate this further we plot the observed correlation between the signal (DF) and the normalization (DB) in Fig. 4.4. To this end, we define the DF relative fluctuation $\Delta_{s}$ as the relative fit residuals at a particular laser detuning. Correspondingly, we define the DB relative fluctuations $\Delta_{b}$ as the observed DB count rate divided by the mean DB count rate of the line scan. The Pearson linear correlation coefficient $\rho\left(\Delta_{s}, \Delta_{b}\right)$ of these two quantities is given by

$$
\rho\left(\Delta_{s}, \Delta_{b}\right)=\frac{\operatorname{cov}\left(\Delta_{s}, \Delta_{b}\right)}{\sigma\left(\Delta_{s}\right) \sigma\left(\Delta_{b}\right)}
$$

where $\operatorname{cov}\left(\Delta_{s}, \Delta_{b}\right)$ is the covariance and $\sigma\left(\Delta_{s}\right)$ and $\sigma\left(\Delta_{b}\right)$ are standard deviations. In this way we obtain an average correlation coefficient for all measured spectroscopic 
lines as stated in figure 4.4 .

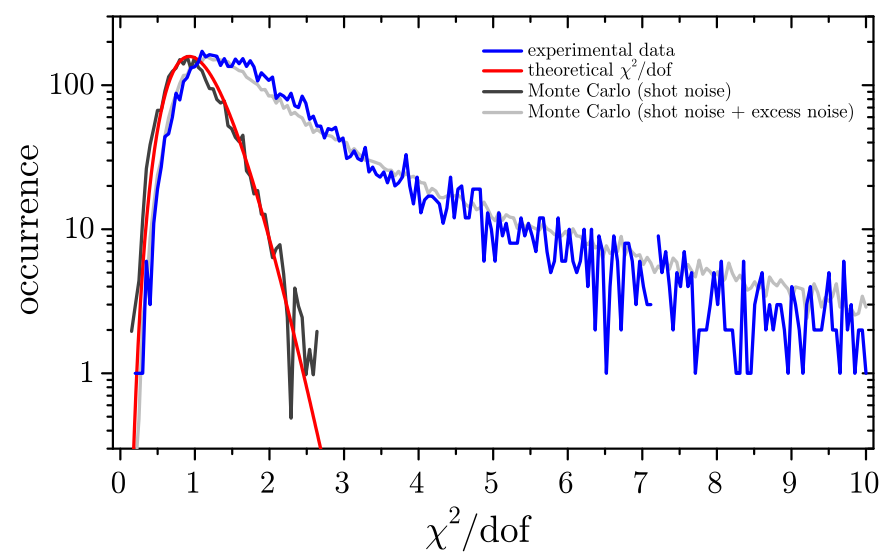

Figure 4.3.: Experimentally obtained distribution of $\chi^{2} /$ dof (black) assuming shot noise as the only noise source and the theoretical $\chi^{2} /$ dof distribution (red). The grey lines are Monte Carlo simulations assuming shot noise only and the observed less-than-perfect correlation between the signal (DF) and normalization (DB).

To verify that this non-perfect correlation can solely explain the observed deviations of the distribution of $\chi^{2} /$ dof values from the expected $\chi^{2}$-distribution, we run a Monte Carlo simulation in the following way: for every recorded line scan we generate a number (16) of artificial lines by using the fit results and adding Poissonian shot noise. Likewise artificial DB signals are generated by adding shot noise to the observed mean values. In addition, we prepare for every artificial line two normally distributed random variables $g_{1,2}$ that are correlated with an adjustable correlation $\rho\left(g_{1}, g_{2}\right)$. These random variables are constructed to have a unity mean and standard deviations that are identical to the standard deviations of $\Delta_{s}$ and $\Delta_{b}$ of the corresponding line scan. The generated DF and DB count rates are multiplied by $g_{1}$ and $g_{2}$ respectively in order to simulate laser intensity noise and fluctuations of the atomic flux. By treating the artificial data in the same way as the real data (normalization, shot noise assumption), we find that a distribution of $\chi^{2} /$ dof values that agrees very well with the experimental distribution (grey line in Fig. 4.3) for $\rho\left(g_{1}, g_{2}\right)=0.9867$. Note that this correlation coefficient is slightly larger than the experimentally observed one because even shot noise, that appears uncorrelated in the DF and DB channels, reduces their correlation. For comparison we also set $\rho\left(g_{1}, g_{2}\right)=1$ and obtain a distribution very close to the theoretical $\chi^{2} /$ dof-distribution (dark grey). Having understood the origin of the additional noise and assuming that it does not give rise to a systematic shift, we scale the uncertainties of the absolute frequencies by $\sqrt{\chi^{2} / \text { dof }}$ if the corresponding line scans obtained $\chi^{2} /$ dof $>1$. However, we do not scale the uncertainties of the other parameters, since these are dominated by other processes than shot noise.

To investigate the signal-to-noise ratio of the data set and to set limits on possible tiny line distortions discussed in section 5.9, we have generated the line shown in 


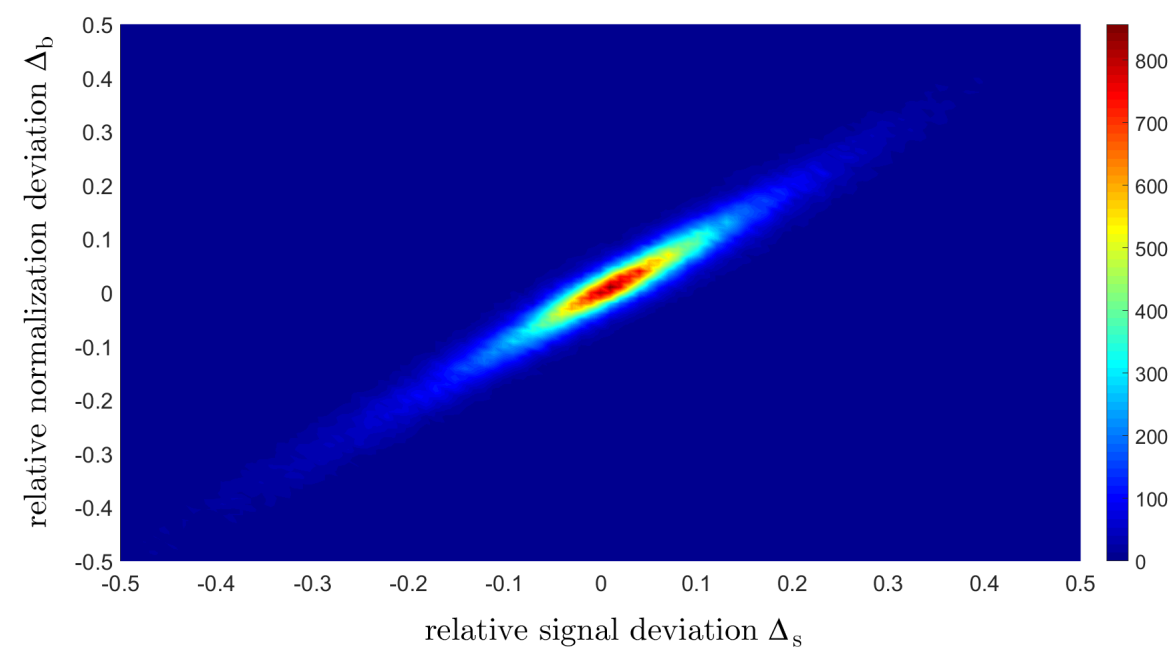

Figure 4.4.: Density plot of the Doppler-free signal count rates (DF) vs. the normalization (DB) count rates. The correlation is very good, as required, but not perfect. From this data the linear correlation coefficient computes to $\rho\left(\Delta_{s}, \Delta_{b}\right)=0.9754$.

Fig. 4.5 by superimposing all 1174 line scans obtained with set temperature of $T=$ $7 \mathrm{~K}$ and a nozzle PCV distance of $d=27.1 \mathrm{~mm}$. To do this we have corrected for the systematic shifts that are described in section 5 and that would otherwise make the line centers to disagree.

\subsection{Line width}

The main broadening mechanism is due to the time-of-flight through the pulse collision volume (PCV). As shown in Fig. 4.6, the observed line width is different for the three detectors. The narrowest lines are observed with the downstream $(j=2)$ detector, because contributing atoms spend the longest time in the PCV. The effect reverses if a detector would be positioned even further downstream such that only the fastest atoms have not decayed when they come into sight. However, in our geometry this mechanism does not dominate. Due to a properly chosen pulse duration, we reach an average observational line width of $0.9980(36) \mathrm{MHz}$ with the downstream detector $(j=2)$ with a nozzle temperature of $4.5 \mathrm{~K}$. With the main detector, the average observational line width is $1.164(52) \mathrm{MHz}$ at $7 \mathrm{~K}$. This means we reach the natural line width of $1.0050 \mathrm{MHz}$, which is computed with approximately 5 digits accuracy in [59] without taking the hyperfine structure into account. The expected difference of the decay rates of the $3 \mathrm{~S}(F=1)$ and $3 \mathrm{~S}(F=0)$ is of the relative order of $10^{-7}$ [60].

Figure 4.6 also shows the line widths predicted with the Monte Carlo simulation as described in section 2.5. Precise modeling of the time-of-flight broadening requires very good knowledge of the geometry and the spatial distribution of the atomic 


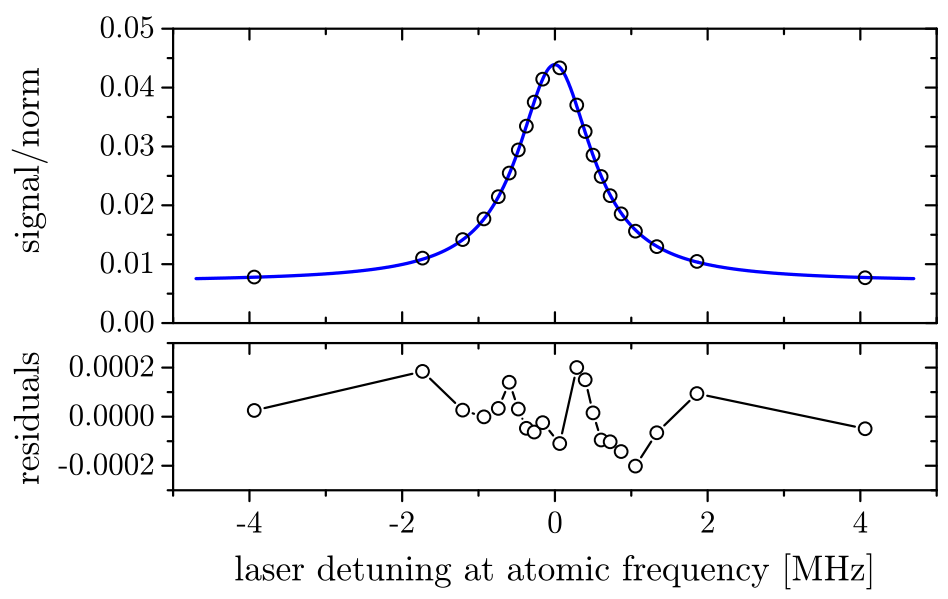

Figure 4.5.: The upper part shows the average of all data that was recorded at a temperature of $7 \mathrm{~K}$ with a pulse collision volume distance to the nozzle of $d=27.1 \mathrm{~mm}$. The systematic shifts due to the CIFODS and the AC-Stark effect has been compensated for each of the contributing lines as described in section 4.4. The distance between the nozzle and the pulse collision volume was fixed and therefore both the pressure shift and the second order Doppler shift were constant. Because of the drift of the lasers repetition rate, the frequency sampling is not exactly the same for the different line scans. Nevertheless, these sampling points have been treated to be identical in this plot. Thanks to the large number of data points, the error bars are much smaller than the markers (about $10^{-3}$ of the signal). The lower plot shows the residuals after fitting a Lorentzian (blue curve).

trajectories. Because of the large aspect ratio of the pulse collision volume $\left(c \tau / w_{0}=\right.$ 6.4), the time-of-flight broadening is much larger for atoms that move at a large angle with respect to the laser beam axis. Hence, the observed line width depends critically on this angle. Our Monte Carlo simulation does not include deflection of atoms due to collisions that might lead to larger crossing angles for some trajectories.

Smaller line broadening effects include collisional broadening, ionization of the $3 \mathrm{~S}$ state, and inhomogeneous broadening due to the second-order Doppler shift and spatially varying AC-Stark shift. The collisional broadening is expected to be of the same magnitude as the collisional shift [67] and is estimated in section 5.4 and table 5.1. Ionization effectively decreases the lifetime of the $3 \mathrm{~S}$ state. From the mean power level given in section 5.2, we estimate a time-averaged laser intensity of $6.1 \mathrm{MW} / \mathrm{m}^{2}$ and obtain an ionization broadening of $120 \mathrm{~Hz}$ with the ionization coefficient given in [30]. The broadening due to the second-order Doppler shift and spatially varying AC-Stark shift are fractions of the associated shifts that are given table 5.1 and hence are expected to be at the $\mathrm{kHz}$ level. Except for the collisional broadening, these broadening mechanisms are included in our Monte Carlo simulation. Since the experimental data reaches the natural line width, it is safe to assume that the collisional broadening is small. A very small contribution to the line width arises from stray electric fields as discussed in section 5.5 and from possible delay line 
misalignments as discussed in section 3.2.

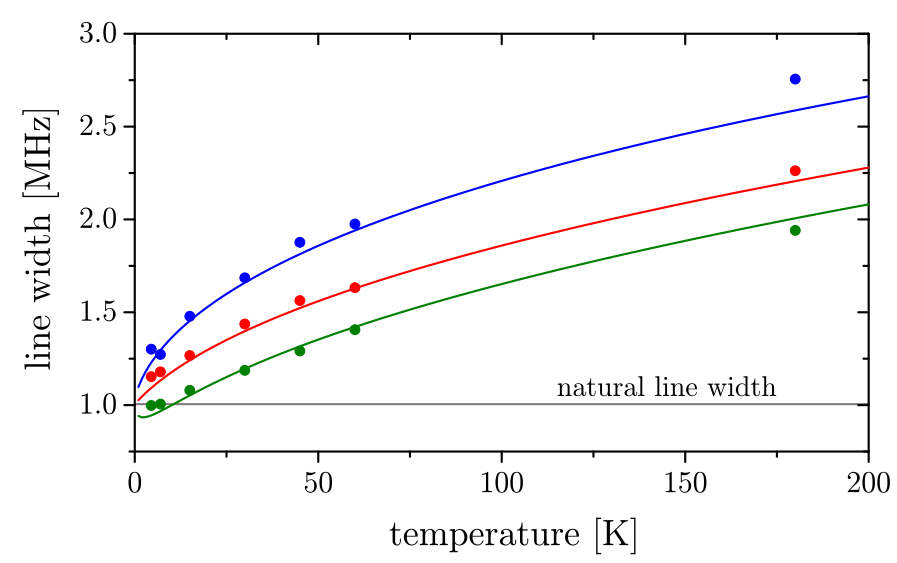

Figure 4.6.: Average line widths determined from Lorentz fits to all experimental data (circles) recorded with a nozzle to pulse collision volume distance of $d=27.1 \mathrm{~mm}$. The red $(j=1)$, green $(j=2)$ and blue $(j=3)$ data points belong to the main and the two auxiliary detectors respectively (see Fig. 3.9). The main broadening mechanism is the time-of-flight broadening. Therefore the light recorded further away from the nozzle $(j=2)$, leads to narrower lines, which reach the natural line width of $1.0050 \mathrm{MHz}$ for the lowest temperatures. The solid lines are obtained from Monte Carlo simulations using Sech-pulse shape. The measured spectrum is not exactly Sech or Gaussian and the theoretical line width depends critically on these details as well as on the exact detection geometry. We have varied the design fiber positions and core diameters with their expected tolerances improve the agreement with the experimental data. This is only to demonstrate the observations are compatible with the modeling within the dimensional tolerances. The data analysis does not depend on the exact positions or core diameters of the fibers.

\subsection{Global fitting procedure}

As described in the previous section, each of the $i=1 \ldots 4450$ valid line scans results in a value for the center frequency obtained with the main detector $f_{1, i}$ as well as with the two auxiliary detectors $f_{2, i}$ and $f_{3, i}$ (parameter $\nu_{0}$ in (4.1)) along with their respective uncertainties $\sigma_{1, i}, \sigma_{2, i}$ and $\sigma_{3, i}$. To include uncertainties due to the limited knowledge of the velocity distribution (deviations from the Maxwellian), we add the full range of second-order Doppler shifts obtained with various distributions (see Fig. 5.9) in quadrature to the frequency uncertainty of $f_{1, i}$ (see section 5.3 for details). We do not expand the uncertainties of $f_{2, i}$ and $f_{3, i}$, because only their differences are used that are mostly free of model uncertainties (see below). For each line scan, we also determine the average cavity transmitted laser power $P_{i}$ with its uncertainty $\sigma_{P, i}$ and the average out-of-loop measured nozzle temperatures $T_{i}$ with uncertainty $\sigma_{T, i}$. The nozzle temperature has been set to $T=4.5 \mathrm{~K}, 7 \mathrm{~K}, 15 \mathrm{~K}, 30 \mathrm{~K}$, $45 \mathrm{~K}, 60 \mathrm{~K}$ and $180 \mathrm{~K}$. Moreover, each line belongs to either of the two distances 
$d_{1}=19.1 \mathrm{~mm}$ or $d_{2}=27.1 \mathrm{~mm}$ between the nozzle back orifice (see section 5.4 ) and the center of the PCV - both with uncertainties of about $0.1 \mathrm{~mm}$.

As described in section 3.6 and detailed in section 5.1, the residual first-order Doppler effect (CIFODS) is proportional to $\beta_{i} \equiv f_{2, i}-f_{3, i}$. The two auxiliary detectors have stronger dependence on CIFODS, but with opposite sign. Since $\beta_{i}$ appears with two signs in the data set, we interpolate rather than extrapolate to $\beta_{i}=0$. This is a very powerful method to remove the CIFODS. The AC-Stark shift (see section 5.2) is proportional to the intracavity laser power and it can be extrapolated to zero using $P_{i}$. Likewise the pressure shift, that is described in section 5.4 , is extrapolated. Instead of using distances $d_{1}$ and $d_{2}$ to quantify the particle density, it is better to use the fitted line amplitudes $a_{i}$ (parameter $a$ in (4.1)), because the flux from the nozzle is also fluctuating. Moreover, the pressure shift is quadratic in $d_{i}$ but it is linear in $a_{i}$. The distance between nozzle and PCV is only known up a unknown offset that changes slightly as the set-up cools down (see section 5.4). The quadratic extrapolation would depend on this offset.

After normalization by the Doppler-broadened (DB) signal, the combined laser power and atomic flux fluctuations are actually divided out. However, we can reintroduce the atomic flux dependence by scaling the line amplitudes via $A_{i}=a_{i} /\langle a\rangle$ with $\langle a\rangle$ being the weighted mean of the line amplitudes $a_{i}$. To maintain the particle density dependence, these averages are computed for the longer distance $d_{2}$ only. To have the same flux calibration for $d_{1}$ and $d_{2}$, the DB detectors have been mounted on the same translation stage that shifts the nozzle relative to the PCV. Moreover, the amplitude scaling needs to take care of the temperature dependence of the $\mathrm{DB} / \mathrm{BF}$ ratio. The total flux has a strong temperature dependence (see Fig. 3.8) and the DF signal is affected by the time-of-flight broadening while the DB is not. Therefore we compute $\langle a\rangle$ separately for each nozzle temperature. The total atomic flux was constantly monitored (see section 3.5) and found to be rather stable. Scaling the amplitudes with the remaining flux variation could be employed but it was found that that final result depends very litte on these details since the pressure shift is a small correction anyway (see section 5.4). The second-order Doppler shift (detailed in section 5.3) is proportional to the square of the atomic velocity and, hence, is in first-order linear with temperature. To take all of these shifts into account, we fit the linear function

$$
f_{1, i}=f_{0}+\kappa_{D S} \beta_{i}+\kappa_{S O D} T_{i}+\kappa_{A C} P_{i}+\kappa_{P S} A_{i}
$$

to the results of all valid line scans simultaneously. The parameters are the unperturbed transition frequency $f_{0}$ and the coefficients for the CIFODS, the second-order Doppler, the AC-Stark and the pressure shift $\left(\kappa_{D S}, \kappa_{S O D}, \kappa_{A C}\right.$ and $\kappa_{P S}$ respectively). While the measured frequencies $f_{1, i}$ depend by construction linearly on these systematic effects, the measured quantities $\beta_{i}, T_{i}, P_{i}$ and $A_{i}$ show very little correlation (see section 5.11). In particular the amplitudes $A_{i}$ are independent of the laser power because they are normalized with the Doppler-free signal. To fit this function to the 
data we minimize

$$
\chi^{2}=\sum_{i} \frac{\left(f_{1, i}-f_{0}-\kappa_{D S} \beta_{i}-\kappa_{S O D} T_{i}-\kappa_{A C} P_{i}-\kappa_{P S} A_{i}\right)^{2}}{\sigma_{1, i}^{2}+\kappa_{D S}^{2} \sigma_{\beta, i}^{2}+\kappa_{S O D}^{2} \sigma_{T, i}^{2}+\kappa_{A C}^{2} \sigma_{P, i}^{2}+\kappa_{P S}^{2} \sigma_{A, i}^{2}},
$$

with the sum over $i$ extending over all valid line scans. Since we have uncertainties on all variables, the weights in the denominator include not only the uncertainties of the ordinate $\left(f_{1}\right.$-axis) but also the ones of the abscissas $(\beta$-axis, $T$-axis, $P$-axis and $A$-axis). Therefore the minimization problem becomes nonlinear and we employ numerical methods (simplex algorithm). Adding the uncertainties in quadrature may or may not require one to take the covariances between the ordinate and abscissas into account. In general the denominator should also contain the covariances between all other measured values. There are two limiting cases: i) Pure instrumental errors, i.e. the actual measured value is absolutely stable, but the instrument adds noise to the reading. In this case no covariance between instruments is expected. ii) A perfect instrument measures a real fluctuation. In that case this fluctuation simply increases the span of the data. No covariance (not even a variance) is expected in this case. In the current situation the noise of $f_{1, i}, f_{2, i}$ and $f_{3, i}$ is of type i) (instrumental shot noise, laser power, and atomic flux). The power measurement $P$ on the other hand is of type ii), since the laser powermeter is much more stable than the actual power fluctuations. However, the line-scan-averaged laser power $P_{i}$ comes with a variance $\sigma_{P, i}^{2}$ that is obtained from the data. The same considerations apply to the temperature measurements.

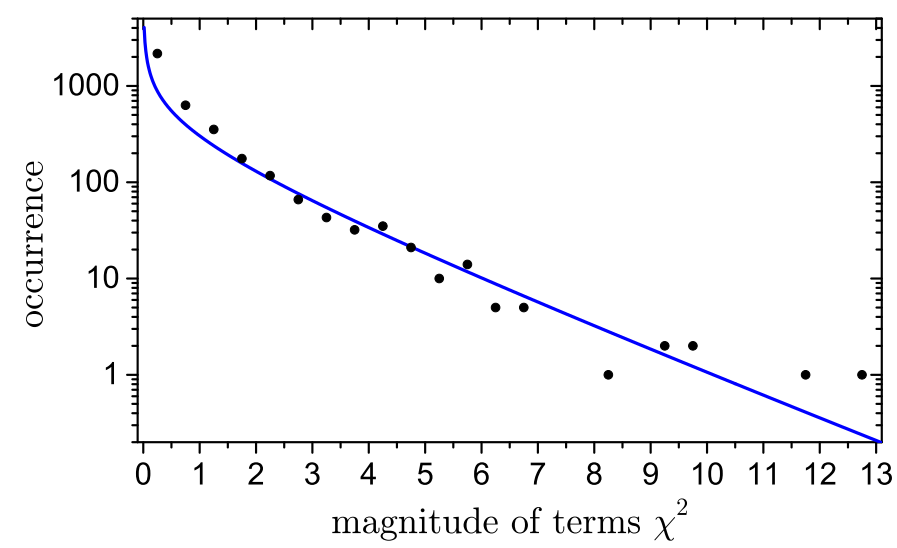

Figure 4.7.: Histogram of all terms that enter the sum in (4.4) with optimized parameters. It closely follows the expected $\chi^{2}$-distribution (solid line) with not more than the expected number of outliers.

As detailed in section 5.3 we expect the largest deviation from the linearity assumption in (4.3) as being due to deviations from the ideal Maxwellian velocity distribution of atoms. This is difficult to quantify for higher temperatures so we decided to limit our data evaluation to the 3681 line scans that where recorded with 
$T \leq 30 \mathrm{~K}$. We use the higher temperature data only as a consistency check. Minimization of (4.4) gives $\chi^{2} /$ dof $=0.75$ with the optimum values of the parameters $f_{0}, \kappa_{D S}, \kappa_{S O D}, \kappa_{A C}$ and $\kappa_{P S}$ as presented in table 4.1. The uncertainties are found by varying the value of the parameter under consideration until $\chi^{2}$ increases by one, while re-optimizing for the remaining parameters. In general this results in asymmetric error bars, however in the current case, this asymmetry is negligible as the minimum of (4.4) is represented by smooth quadratic functions along all parameter axes. To verify that there are no outliers that would have an over-represented influence on the result, we plot a histogram of all terms that enter (4.4) with the optimum parameters in Fig. 4.7.

\begin{tabular}{||c|c|c|c|c||}
\hline$f_{0}$ & $\kappa_{D S}$ & $\kappa_{S O D}$ & $\kappa_{A C}$ & $\kappa_{P S}$ \\
\hline \hline$-0.57(55) \mathrm{kHz}$ & $-0.0437(43)$ & $-315(25) \mathrm{Hz} / \mathrm{K}$ & $139.7(9.1) \mathrm{Hz} / \mu \mathrm{W}$ & $0.58(22) \mathrm{kHz}$ \\
\hline
\end{tabular}

Table 4.1.: The results of the global minimisation of (4.4) with a minimum $\chi^{2} /$ dof $=0.75$ limiting data recorded with temperatures of $T \leq 30 \mathrm{~K}$. The transition frequency $f_{0}$ is relative to the final result, i.e. additional frequency shifts of a total of $0.57 \mathrm{kHz}$ are not included here but listed in table 5.1. Including the $T>30 \mathrm{~K}$ data for the evaluation shifts the resulting $f_{0}$ by only $-13 \mathrm{~Hz}$.

A lot of effort was necessary to experimentally investigate the systematic effects. We varied key parameters to extrapolate the second-order Doppler, AC-Stark and pressure shift and interpolate the CIFODS. As a result, the statistics were somewhat degraded - the statistical uncertainty of the data without extrapolations is $70 \mathrm{~Hz}(108 \mathrm{~Hz}$ if only the main detector is used), while after extrapolations the final uncertainty is $0.55 \mathrm{kHz}$. This is a robust procedure since it is largely independent of modeling. We use Monte Carlo simulations mostly for cross checks and to estimate systematic shifts. Only a small correction due to quantum interference solely relies on Monte Carlo simulations (see section 5.8). Systematic shifts due to external fields (DC-Stark and Zeeman) do not need to be evaluated for every line scan. Instead, it was only necessary to compensate these fields several times during the whole campaign (see section 5.5 and 5.6). 


\section{Systematic Uncertainties Analysis}

\subsection{Chirp induced residual first-order Doppler shift}

Two-photon transitions allow for the suppression of the first-order Doppler shift. However, in direct frequency comb two-photon spectroscopy a residual chirp induced first-order Doppler shift (CIFODS) may emerge [21]. In our case, the divergence of the atomic beam, together with a small frequency chirp of the laser pulses causes this shift and is explained below. For an approximate analytical model we are assuming a Gaussian pulse shape because it leads to compact expression, while we can readily use any pulse shape for the Monte Carlo simulations. Consider two counter-propagating linearly chirped pulse trains,

$$
E_{ \pm}=E_{0} \sum_{n} \exp \left(-(1+i b) \frac{\left(t-n t_{\mathrm{rep}} \pm z / c\right)^{2}}{\tau^{2}}-\frac{x^{2}+y^{2}}{w(z)^{2}}-i \omega_{c} t \mp i k_{c} z \pm i \arctan \left(z / z_{R}\right)\right)
$$

with the position along the propagation direction $z$, the radial position $x, y$, time $t$, the usual beam radius $w(z)=w_{0} \sqrt{1+\left(z / z_{R}\right)^{2}}$, the Rayleigh length $z_{R}=k_{z} w_{0}^{2} / 2$, and the laser beam waist $w_{0}$. The sum extends over the forward $\left(E_{+}\right)$and backward $\left(E_{-}\right)$ propagating pulses with the repetition time $t_{\text {rep }}=1 / f_{\text {rep }}$, pulse duration $\tau$ (FWHM intensity $T_{1 / 2}=\tau \sqrt{2 \ln (2)}$ ), carrier frequency $\omega_{c}=c k_{c}$, and the dimensionless chirp parameter $b$. We choose a pure frequency domain chirp (in contrast to a time domain chirp, see [26] with $a=1$ ), because this is what we expect with ps pulses and the dominating self-phase modulation. The reversing sign of the $z / c$ term is given by requiring the same temporal color sequence of the reflected pulses. The Rabi frequency of the (almost) Doppler-free transition amplitude is proportional to the product of the two fields $E_{+} E_{-}[30]$

$$
\Omega(t)=\left(\frac{2}{\pi}\right)^{3 / 2} \frac{8 \pi \beta_{g e} P_{L}}{f_{\mathrm{rep}} \tau w(z)^{2}} \sum_{n} \exp \left(-2(1+i b)\left[\frac{\left(t-n t_{\mathrm{rep}}\right)^{2}}{\tau^{2}}+\frac{z^{2}}{c^{2} \tau^{2}}\right]-2 \frac{x^{2}+y^{2}}{w(z)^{2}}-i 2 \omega_{c} t\right),
$$

where we consider only one PCV close to $z=0$ and are therefore left with only one sum. One can also observe that a power imbalance between the forward and backward propagating pulses $\left(\left|E_{+}\right| \neq\left|E_{-}\right|\right)$does not lead to a Doppler shift but only a decrease in the Doppler-free transition amplitude. The Rabi frequency of the Doppler-broadened component is proportional to $E_{+} E_{-}^{*}$. The laser power $P_{L}$ is measured in one propagation direction only and the parameter $\beta_{g e}$ is given in section 2.5. The term that oscillates with twice the carrier frequency is removed with the rotating wave approximation. For an atomic trajectory along the $z$-axis with $z(t)=z_{0}+v_{z} t$, one sees that the usual first-order Doppler term $k_{c} v_{z}$ is absent. 
However, there are two new time-dependent phase terms for $b \neq 0$. The first one, $-2 b\left(t-n t_{\mathrm{rep}}\right)^{2} / \tau^{2}$, does not produce a frequency shift, since it is repetitive with the pulse train. It leaves the frequencies of the comb modes where they are, but reduces the excitation rate [26]. The second phase term $\varphi=-2 b(z / c \tau)^{2}$ however, leads to an instantaneous chirp-dependent frequency shift of $\dot{\varphi}=-4 b v_{z} z /\left(c^{2} \tau^{2}\right)$. Just like the usual first-order Doppler effect, this frequency shift is linear in velocity. However, in contrast to the usual case, this shift also depends on the position $z$ and therefore averages to zero with this simple axial trajectory. The compensation is not perfect if the atomic beam is divergent so that positive and negative values of $z$ enter with different weights to the signal, or if the signal is received from parts of the PCV only. This is illustrated in Fig. 5.1.

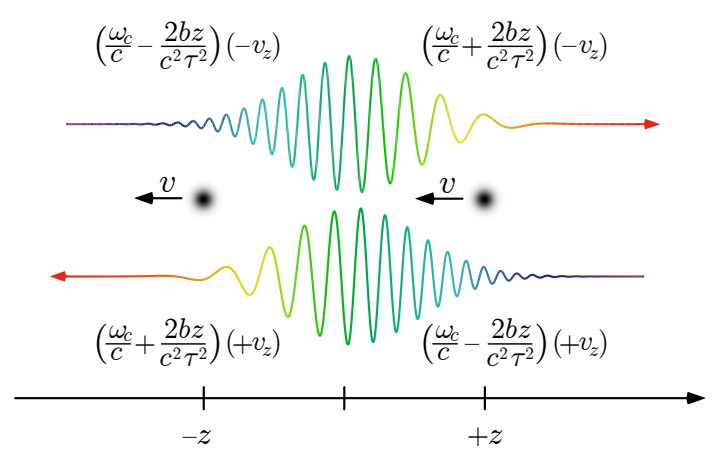

Figure 5.1.: Principle of the "chirp induced residual first-order Doppler shift" (CIFODS). Two chirped counter-propagating pulses with carrier frequency $\omega_{c}$ (sketched spatially separated for clarity) meet within the pulse collision volume, where they drive a two-photon transition in an atom that moves parallel to the $z$-axis with a velocity $v_{z}$. The instantaneous frequency shifts at the edges of the pulses are $\pm 2 b v_{z} z / c^{2} \tau^{2}$ at positions $\pm z$, with the chirp parameter $b$ and with the value of the shift doubling for a two-photon transition. For the Doppler shift, the velocity has to be measured relative to the pulse propagation direction. The shifts cancel for atoms that travel exactly parallel to the $z$-axis. In a divergent atomic beam however, this cancellation is no longer perfect and one might estimate the CIFODS by integrating over $z$ with a weight given by the square of the magnitude of the Rabi frequency $\propto \exp \left(-4(z-d)^{2} / c^{2} \tau^{2}\right)$ with distance $d$ between the PCV and the emerging point of the atomic beam. This results in $\Delta \omega \approx-b v / d$.

To gain more insight into this effect, we consider a general atomic trajectory of the form $\vec{r}(t)=\left(x_{0}+v_{x} t, y_{0}+v_{y} t, z_{0}+v_{z} t\right)$ and Fourier transform the Rabi frequency (5.2). We obtain a comb like structure that is broadened (by time-of-flight) and shifted from the original laser modes by [21]:

$$
2 \pi \delta \nu_{D S}=4 b v_{z} \frac{\left(v_{x} v_{z} x_{0}+v_{y} v_{z} y_{0}-\left(v_{x}^{2}+v_{y}^{2}\right) z_{0}\right)}{v_{z}^{2} w_{0}^{2}+\left(v_{x}^{2}+v_{y}^{2}\right) c^{2} \tau^{2}}
$$

where we assumed the Rayleigh length to be much larger than the length of the 
PCV so that beam radius can be taken as a constant. This shift is measured at the atomic frequency. As expected, the frequency shift vanishes for an axial atomic beam with $v_{x}=v_{y}=0$. A divergent beam, as in our apparatus, experiences a CIFODS. To estimate its magnitude we consider a longitudinal atomic beam that emerges at the nozzle orifice with radius $R_{n}$ located at $\left(x_{0}, y_{0},-d\right)$, at a distance $L$ from the center of the PCV. The atomic velocity in spherical coordinates is $\vec{v}=v(\sin \theta \cos \phi, \sin \theta \sin \phi, \cos \theta)$. We assume that the contribution to the signal is proportional to $\left(\Omega_{0} \Delta t\right)^{2}$, where $\Omega_{0}$ is the Rabi frequency at the center of the PCV and $\Delta t$ is the time-of-flight through the PCV. Averaging over all trajectories and assuming a small angle $\theta\left(d \ll w_{0}, R_{n}, c \tau\right)$, we find

$$
2 \pi \delta \nu_{D S} \approx \frac{b v}{d}
$$

With the most probable thermal velocity of $v_{0}=340 \mathrm{~m} / \mathrm{s}$ at $7 \mathrm{~K}$ (see section 5.3), a distance of $d=19.1 \mathrm{~mm}$, and the mean chirp coefficient of $\langle b\rangle=-0.119$ (see Fig. 5.6), we estimate the CIFODS to be $\delta \nu_{D S} \approx-0.34 \mathrm{kHz}$.

A much more accurate model is obtained with a Monte Carlo simulation, which is described in more detail in section 2.5. It involves solving the optical Bloch equation for all levels up to the principle quantum number $n=3$ with many atomic trajectories randomly chosen from a thermal distribution. It takes all important experimental parameters into account such as the detection geometry and efficiency, laser pulse profiles, and others. The output of this simulation is evaluated in the same way as the experimental data to determine the transition frequencies. Figure 5.2 shows the results of such a simulation for the main detector $j=1$ and the auxiliary detectors with various realistic values of the chirp parameter. If the detector is centered to give the maximum signal, the large field of view detector $j=1$, within the range of $z=-0.5 \ldots+0.5 \mathrm{~mm}$, gives a CIFODS of around $700 \mathrm{~Hz}$ for a change in $b$ of 0.1 .

To compare these simulations with experimental results, we have performed an auxiliary measurement where we varied the position of the light collecting lens, while keeping the chirp, nozzle position and its temperature constant. The measurement has been done only with the large field of view detector $(j=1)$ and the results are shown in Fig. 5.3. The chirp parameter, temperature, laser power and external fields (DC-Stark and Zeeman) have been held as constant as possible but were not characterized. The theoretical curve is adjusted by interpolating between sets of simulations with discrete steps in $b$ and by adjusting a common frequency offset as well as an offset of the lens position $z$, which is difficult to determine precisely.

To compensate for the CIFODS one might consider positioning the light collecting lens at the location where all curves of Fig. 5.2 cross, so that the observed transition frequency becomes independent of the chirp. However, that position is difficult to find experimentally, especially when it is not easy to vary the chirp parameter in a well defined way (see below). In addition, the slope of these curves is large at this point and the accuracy of the Monte Carlo simulations sensitively depends on the knowledge of the experimental geometry. 


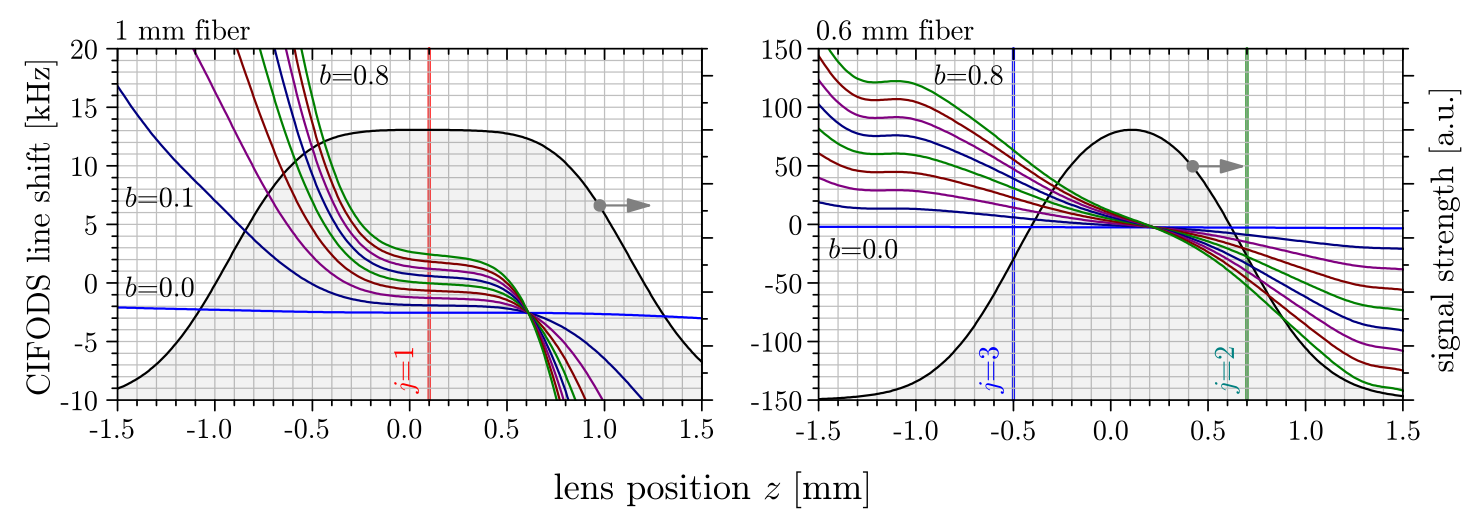

Figure 5.2.: Monte Carlo simulation of the chirp induced first-order Doppler shift (CIFODS) obtained with a Gaussian pulse with a duration of $T_{1 / 2}=\tau \sqrt{2 \ln (2)}=2.0 \mathrm{ps}$, the velocity distribution given in section 5.3 , at a nozzle temperature of $T=7 \mathrm{~K}$, and a nozzle distance of $d=27.1 \mathrm{~mm}$. The shift is measured at the atomic frequency. The horizontal axis gives the position $z$ of the lens that collects the fluorescence signal (see Fig. 3.9 ) with respect to the center of the pulse collision volume (PCV). The left vertical axes give the obtained line center (measured at the atomic frequency by fitting a Lorentzian) relative to the unperturbed transition frequency without the AC-Stark and pressure shift. The different curves correspond to different chirp parameters from $b=0$ to 0.8 in steps of 0.1 . For negative $b$ one would get the corresponding negative shift (see (5.3)). The simulations are done for light collecting multimode fibers with $1 \mathrm{~mm}$ (left) and $0.6 \mathrm{~mm}$ (right) core diameters. The approximate position of the detectors $j=1$ and $j=2,3$ (see section 3.6) are marked with the red, green and blue vertical lines respectively. Experimentally the positions are determined relative to the peak count rate. The right vertical axes show the normalized signal amplitude as seen by the detectors (see Fig. 3.10). The center of the curve is shifted by about $0.1 \mathrm{~mm}$ from $z=0$ due to the finite lifetime of the excited state along with the velocity of the atoms (traveling from left to right in this plot). The almost constant frequency offset for $b=0$ (blue) is due to the second-order Doppler shift (SOD) (see section 5.3). The SOD is not exactly constant, because faster atoms produce more signal further downstream. See section 5.11 for details.

A much better method, which is essentially independent of the simulations, is to measure the CIFODS simultaneously with two auxiliary chirp-sensitive detectors. As described in section 3.6, the additional light collecting lenses are aligned roughly at the ends of the PCV and feed multimode fibers with a smaller core diameter of $0.6 \mathrm{~mm}$. Both of these features makes the recorded lines more sensitive to CIFODS (as one can see from the scales in Fig. 5.2). As the sign of the CIFODS is opposite at opposite ends of the PCV, the (CIFODS) unperturbed line center is found by interpolation. Without relying on accurate modeling, interpolation is much more precise than extrapolation. The exact position of the lenses is irrelevant to ensure a linear interpolation function. Moreover, with this method, the chirp parameter, which is only moderately controlled, needs to be stable during one line scan only. 


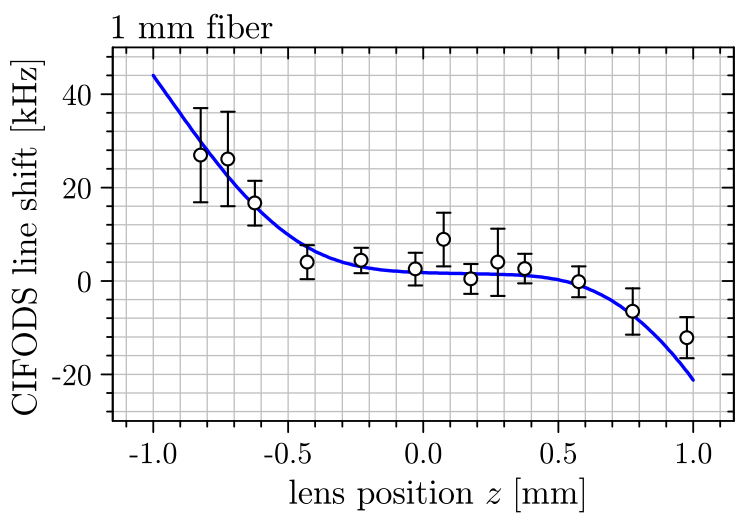

Figure 5.3.: Experimental determination of the chirp induced residual first-order Doppler shift (CIFODS) measured at the atomic frequency and recorded at a nozzle temperature of $8 \mathrm{~K}$. We attempted to keep the other systematic frequency shifts as constant as possible, but they were not characterized. Therefore, the CIFODS shown here is relative to an unknown offset. The error bars are obtained from a weighted average of lines scans that are assumed subject to shot noise only. This plot is to be compared with the theoretical model presented in Fig. 5.2. The latter is adjusted for the chirp parameter $b$, an offset of the lens position $z$, as well as a frequency offset. The best fitting $\left(\chi^{2} /\right.$ dof $\left.=0.55\right)$ chirp coefficient is $b=0.5(1)$, which corresponds to a CIFODS of $3.5 \mathrm{kHz}$ with a centered lens.

Further, as explained below, we do not even need to know its value. As seen at the right hand side of Fig. 5.2, the CIFODS at $z=-0.5 \mathrm{~mm}$ is about $8 \mathrm{kHz}$ for a change of $\Delta b=0.1$. At the opposite side at $z=0.7 \mathrm{~mm}$ (note that the signal maximizes at $z=0.1 \mathrm{~mm})$, the dependence is slightly smaller $(6.5 \mathrm{kHz}$ per 0.1 change of the chirp parameter). For example, by placing the main detector at $z=0.1 \mathrm{~mm}$, and the auxiliary detectors at $z=-0.5 \mathrm{~mm}$ and at $z=0.7 \mathrm{~mm}$, the frequency difference observed by the two auxiliary detectors is about $15 \mathrm{kHz}$ for a chirp of $b=0.1$ with the $0.6 \mathrm{~mm}$ fibers. This is roughly 10 times larger than for the main detector.

Even though the CIFODS is a complicated function of the position $z$, for fixed $z$ it is very linear as a function of the chirp parameter $b$ as the simulations in Fig. 5.4 show. This can be exploited with a linear interpolation method for its compensation. For this purpose we first express the observed transition frequencies $f_{j, i}$ obtained with the detectors $j=1,2,3$ and the line scan $i$ in terms of the CIFODS unperturbed transition frequency $f_{0}$ and three (unknown) coefficients $\alpha_{j}$ :

$$
f_{1, i}=f_{0}+\alpha_{1} b_{i} \quad f_{2, i}=f_{0}+\alpha_{2} b_{i} \quad f_{3, i}=f_{0}+\alpha_{3} b_{i} .
$$

Then these equations are rearranged in the following way

$$
f_{1, i}=f_{0}+\frac{\alpha_{1}}{\alpha_{2}-\alpha_{3}}\left(f_{2, i}-f_{3, i}\right)=f_{0}+\kappa_{D S}\left(f_{2, i}-f_{3, i}\right)=f_{0}+\kappa_{D S} \beta_{i}
$$

which gives us the linear interpolation model that has been used in section 4.4 with $\beta_{i} \equiv f_{2, i}-f_{3, i} \propto b_{i}$. The actual value of the chirp parameter is not required to find 

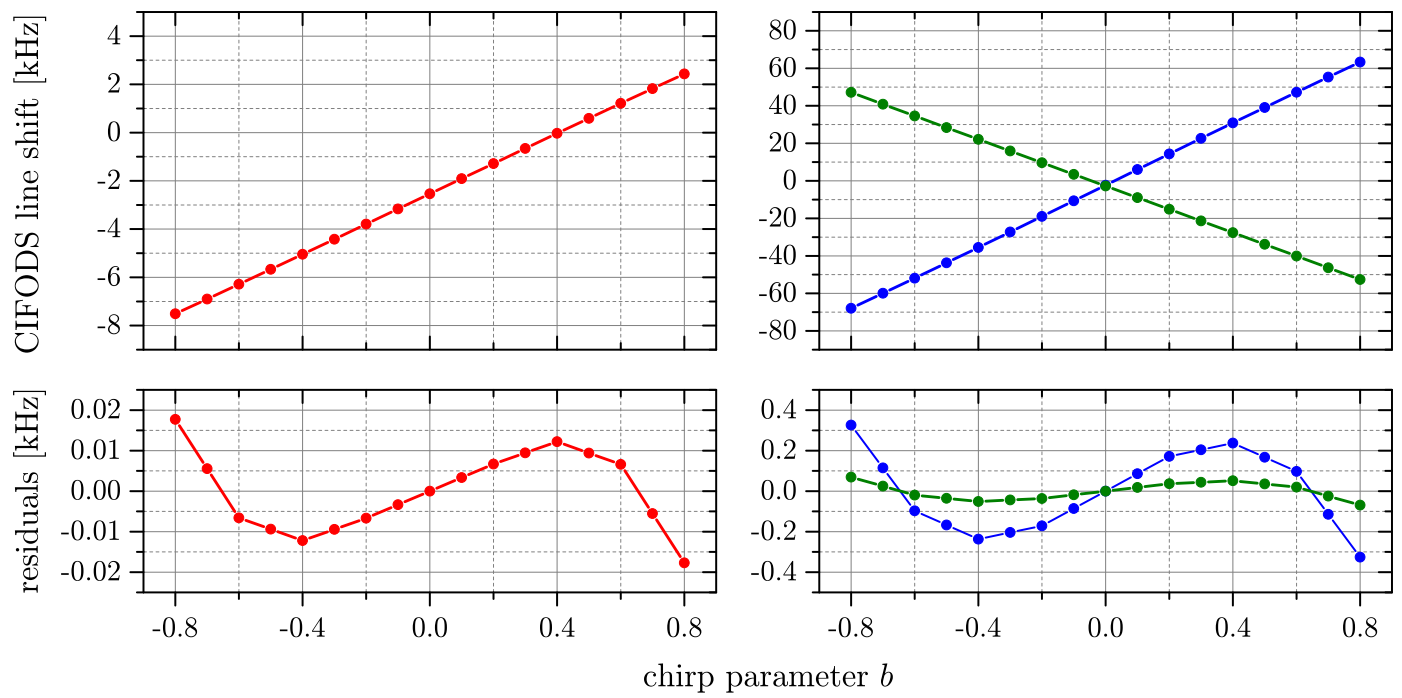

Figure 5.4.: The same Monte Carlo data as in Fig. 5.2 but plotted as a function of the chirp parameter $b$ instead of the lens position $z$. Left: Predicted CIFODS obtained with the main detector $j=1$ at $z=+0.1 \mathrm{~mm}$ that is fed by a $1 \mathrm{~mm}$ multimode fiber. The dependence on the chirp parameter is linear up to the residuals shown at the bottom. The offset at $b=0$ is due to the second-order Doppler shift. Right: The CIFODS seen with the auxiliary detectors $j=2$ and $j=3$ positioned at $z=-0.5 \mathrm{~mm}$ and $z=+0.7 \mathrm{~mm}$ is a factor of 23 larger $\left(=1 / \kappa_{D S}\right)$ because these detectors are fed with a $0.6 \mathrm{~mm}$ multimode fiber. Again the CIFODS is reasonably linear with a frequency difference of about $145 \mathrm{kHz}$ for a change of the chirp parameter of $\Delta b=1$. The deviation from the linearity as presented at bottom, is at most $400 \mathrm{~Hz}$ for this simulation. From this we estimate the systematic uncertainty due to the nonlinearity of the CIFODS on the main detector to be a factor 23 smaller, i.e. at a negligible level.

the intercept. For true interpolation, it is required that we experimentally operate with $\beta_{i}$ values of both signs, which is indeed the case as shown in Fig. 5.5.

Because $f_{2, i}$ and $f_{3, i}$ are strongly anticorrelated (by construction), its variance is given by:

$$
\sigma_{f_{2, i}-f_{3, i}}^{2}=\sigma_{f_{2, i}}^{2}+\sigma_{f_{3, i}}^{2}-2 \operatorname{cov}\left(f_{2, i}, f_{3, i}\right) .
$$

The covariance enters with a negative sign because of the frequency difference. We estimate it with the Pearson correlation coefficient $\rho\left(f_{2}, f_{3}\right)$ multiplied with the variances of the line center, i.e. $\operatorname{cov}\left(f_{2, i}, f_{3, i}\right)=\rho\left(f_{2}, f_{3}\right) \sigma_{f_{2, i}} \sigma_{f_{3, i}}$.

From the full data set, we find $\rho\left(f_{2}, f_{3}\right)=-0.5695$ so that the anticorrelation increases the uncertainty. From the global fit described in section 4.4 we find $\kappa_{D S}=$ -0.0437(43) (see table 4.1). This parameter may also be extracted from our Monte Carlo simulations. With a nozzle temperature of $7 \mathrm{~K}$ and a nozzle distance of $d=27.1 \mathrm{~mm}$ we obtain $\kappa_{D S}=-0.043$ (as seen in Fig. 5.4 as the slope of the left plot divided by the difference of the slopes of the right plot), which is in very good agreement with the experimental observation. Using the experimental data and the 


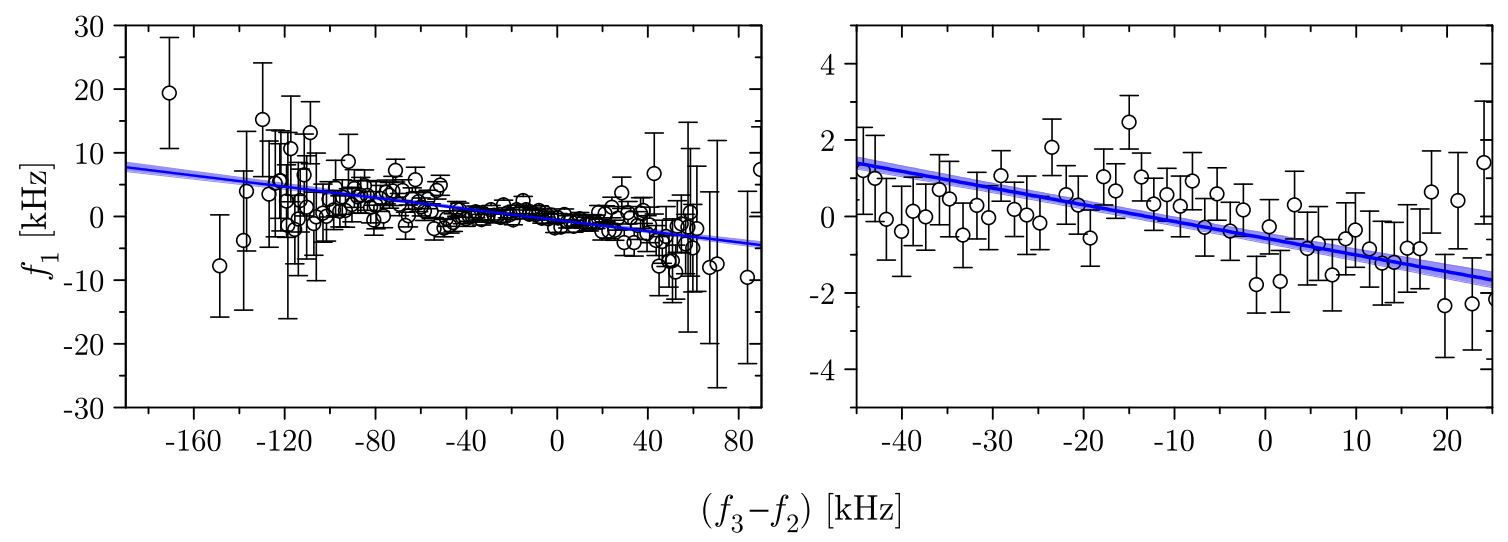

Figure 5.5.: Chirp induced first-order Doppler shift (CIFODS) measured at the atomic frequency relative to the final result of this work determined by correcting each line scan for the second-order Doppler $\left(\kappa_{S O D}\right)$, the AC-Stark $\left(\kappa_{A C}\right)$ and the pressure shift $\left(\kappa_{P S}\right)$ using the results of the global fit in table 4.1 (additional corrections as listed in table 5.1 are not applied in this graph). The linear function is the result of the global fit with $\kappa_{D S}=-0.0437(43)$ taken from the same table that is shown together with the $1 \sigma$ confidence interval bands. Left: all data; right: zoom into the most significant smallerror-bar region that covers a range of about $80 \mathrm{kHz}$ for $f_{3}-f_{2}$. For clarity the data has been binned into $1.8 \mathrm{kHz}$ intervals on the horizontal axis for the plot only - not for the global fit. The error bars are obtained from a weighted average of lines scans within each bin assuming these line scans are subject to shot noise only. The uncertainties of $f_{2}-f_{3}$ have been omitted in the plot for clarity, but are used for the global fit.

Monte Carlo simulation, we can estimate the chirp parameter for each line scan. A histogram of all chirp parameters is displayed Fig. 5.6.

The nonlinearity seen in the residuals of Fig. 5.4 give rise to an additional uncertainty of the $\beta_{i}$ on the order of about $400 \mathrm{~Hz}$ for the auxiliary detectors. This enters (5.7) with a factor $\kappa_{D S}$ which reduces it to a negligible level of $20 \mathrm{~Hz}$. The CIFODS parameter determined in this way, receives a small contribution from the second-order Doppler shift, because faster atoms contribute more signal downstream from the nozzle. Restricting the evaluation to temperatures up to $30 \mathrm{~K}$ limits the influence of this cross-talk to the $\mathrm{Hz}$ level (see section 5.11 for details).

For visualization of the magnitude of the CIFODS in our experiment, we need to separate it from the other corrections. For this purpose, we correct line scans for all shifts except for the CIFODS using the results of the global minimization. Figure 5.5 shows the results.

As mentioned in section 3.2, the chirp of the laser pulses can be manipulated by longitudinally shifting the BBO crystal in the second doubling stage. We do this occasionally between some of the line scans. Unfortunately, this is not a deterministic method to adjust for a certain value of $b$. Nevertheless, it is sufficient to cover a large enough range of the parameter $\beta$ to interpolate to $\beta=0$ (see Fig. 5.5).

The discussion in this section so far was limited to a special type of pulse chirp 


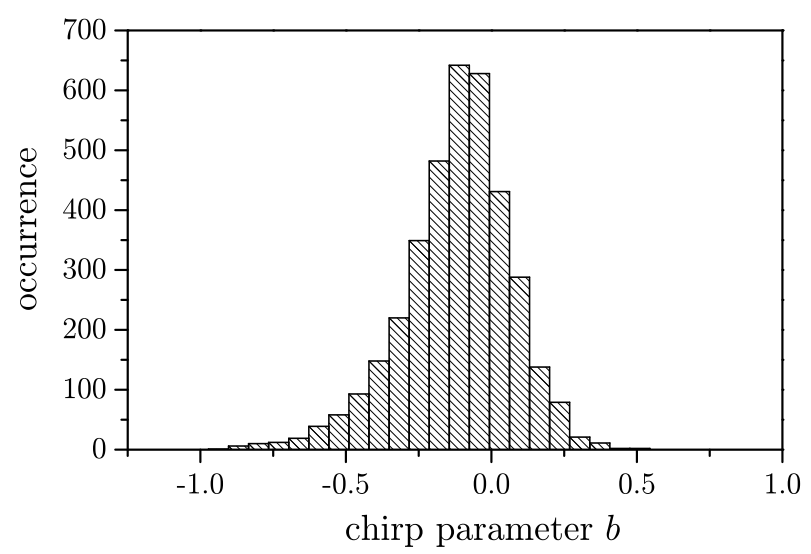

Figure 5.6.: Distribution of chirp parameters extracted from the experimental data shown in Fig. 5.5. The linear mapping of the measured difference $f_{3}-f_{2}=0$ on $b$ is done by identifying $f_{3}-f_{2}=0$ with $b=0$ and by using the slope from the Monte Carlo simulation shown in Fig. 5.4 for $T=7 \mathrm{~K}$. Different slopes (approximately $\propto \sqrt{T}$ ) have been used for the other nozzle temperatures. In this way we can estimate the chirp parameter for each line scan and generate this histogram. The distribution has a FWHM of about $\Delta b=0.38$ and is not quite Gaussian (and also not expected to be a Gaussian). The mean values obtained from this distribution are $\langle b\rangle=-0.119(17)$ and $\left\langle 1 / \sqrt{1+b^{2}}\right\rangle=0.9783(31)$, where the uncertainties are estimated from the variance of the distribution.

- that is a linear frequency sweep (or quadratic phase sweep) as expressed by (5.1). We now show that the interpolation method is robust against higher order phase terms, provided the chirp function can be characterized by a single parameter. The pulse chirp may be given by a more general phase function $\phi(t \pm z / c)$, which would be $\phi(t \pm z / c)=-b(t \pm z / c)^{2} / \tau^{2}$ for the simple linear frequency sweep considered so far. We assume that this phase function is periodic with the pulse train and that it should not be computed for arguments that are much larger than the pulse duration. We therefore consider the interaction of an atom with an individual pulse as it flies through the PCV by expanding the phase function in a power series around $t=0$ as $\phi(t)=\sum_{m}=b_{m}(t / \tau)^{m}$. As a constant phase has no effect in the spectroscopy and a linear phase is simply a frequency offset of the whole comb (that is measured, see section 3.1), this series starts with $m=2$. After the rotating wave approximation, the Rabi frequency (5.2) close to the pulse center at $t=0$ becomes:

$$
\begin{array}{r}
\Omega(t) \propto \exp \left(-i \sum_{m=2}^{\infty} b_{m}\left[\left(\frac{t+z / c}{\tau}\right)^{m}+\left(\frac{t-z / c}{\tau}\right)^{m}\right]-\frac{2 t^{2}}{\tau^{2}}-\frac{2 z^{2}}{c^{2} \tau^{2}}-2 \frac{x^{2}+y^{2}}{w^{2}}\right) \\
=\exp \left(-i \sum_{m=2}^{\infty} \sum_{k=0}^{m} b_{m}\left[\left(\begin{array}{c}
m \\
k
\end{array}\right)\left(\frac{t}{\tau}\right)^{m-k}\left(\frac{z}{c \tau}\right)^{k}\left(1+(-1)^{k}\right)\right]\right. \\
-\frac{2 t^{2}}{\tau^{2}}-\frac{2 z^{2}}{c^{2} \tau^{2}}-2 \frac{x^{2}+y^{2}}{w^{2}}(9.8)
\end{array}
$$

We integrate over the Rabi frequency to determine the excited state population that 
is added per pulse:

$$
a(z)=\int_{-n \tau}^{+n \tau} \Omega(t) d t
$$

Here $n \tau$ is some multiple of the pulse duration. For the simulations described in more detail in section 2.5, we use $n=3$ as a good approximation. Increasing $n$ did not yield a significant change of the results. To further simplify the computations, we assume that the atom position is fixed during the pulses, but account for its motion between the pulses. To justify this approach, we note from (5.8) that the phase change of $\Omega(t)$ is proportional to $(t / \tau)^{2}$ to leading order - within a given pulse, we have $|t / \tau| \lesssim n$, whereas between two pulses this number is more than 3 orders of magnitude larger.

Any chirp-induced Doppler shift can now be understood as a phase change of $a(z)$ with position:

$$
\delta \nu_{D S}=\frac{v_{z}}{2 \pi} \frac{\partial \arg [a(z)]}{\partial z} .
$$

The phase term of the integral (5.9) extends over an odd function for odd $m-k$ and hence vanishes. Therefore only even $m-k$ contribute to the chirp, and since only even $k$ occur, $m$ must be an even number as well.

Our main source of chirp is due to self-phase modulation within the doubling crystals (see section 3.2). For this case, the phase function, plotted in Fig. 3.4, is given by:

$$
\phi(t)=\phi_{0} e^{-2 t^{2} / \tau^{2}}=\phi_{0}\left(1-\frac{2 t^{2}}{\tau^{2}}+\frac{2 t^{4}}{\tau^{4}}-\frac{4 t^{6}}{3 \tau^{6}}+\ldots\right) .
$$

Here, the odd terms are not even present and do not need to be suppressed by the twophoton excitation scheme. The additional higher-order terms give rise to a different CIFODS so that the curves in Fig. 5.2 would need to be modified. Nevertheless, we expect that the frequency shift is in first-order linear in the new parameter $\phi_{0}$, similar to Fig. 5.4. In addition, the three detectors will obtain different CIFODS, which is in our geometry always minimal for the detector $j=1$ that has the largest field of view. With these assumptions we can define new (unknown) coefficients $\alpha_{j}^{\prime}$ :

$$
f_{1, i}=f_{0}+\alpha_{1}^{\prime} \phi_{0, i} \quad f_{2, i}=f_{0}+\alpha_{2}^{\prime} \phi_{0, i} \quad f_{3, i}=f_{0}+\alpha_{3}^{\prime} \phi_{0, i},
$$

which is in complete analogy to (5.5), and results in the same interpolation formula (5.6). It should be noted that this argument can be extended to a more general case of phase functions where the coefficients on the right hand side of (5.11) have different values. We do not expect additional systematic CIFODS effects as long as the relative size of the coefficients in that power series do not change between the lines scans and the chirp can be described by only one varying parameter, $\phi_{0, i}$.

With multiple mechanisms that can lead to a chirp, one might be in the regime of a "multi-parameter CIFODS". In this case the coefficients in the power series may change simultaneously and independently. This may happen for example when the mode locked laser contributes a linear chirp $b$ that adds to the chirp that is generated 
by self-phase modulation within the doubling crystals. From the experiment and simulations we know that the CIFODS we observe corresponds to a linear chirp in the range of $b \approx-0.5 \ldots 0.5$. Further we know that most of our chirp is caused by self-phase modulation, so that the coefficient of the next order term, $t^{4} / \tau^{4}$, is at most as large as the coefficient of the first term. To estimate the associated systematic shift we ran Monte Carlo simulations (see section 2.5) with a phase profile $\phi(t)=b_{2} t^{2} / \tau^{2}+b_{4} t^{4} / \tau^{4}$, where the coefficients are varied within the ranges of $b_{2}=$ $0,0.1,0.2, \ldots 0.9$ and $b_{4}=-0.4,0.2,0,0.2,0.4$. By evaluating all combinations of coefficients with the interpolation method, we find a maximum shift of $\pm 0.1 \mathrm{kHz}$. This possible systematic uncertainty is included in the final error budget shown in table 5.1.

\subsection{AC-Stark shift}

The AC-Stark effect can be computed analytically for atomic hydrogen. Both the ground state and the excited state are shifted by the local laser intensity $I(\vec{r})$ according to

$$
\delta \nu_{A C}=2\left(\beta_{A C}(3 S)-\beta_{A C}(1 S)\right) I(\vec{r})=2.565902 \times 10^{-4} \frac{\mathrm{Hz}}{\mathrm{W} / \mathrm{m}^{2}} I(\vec{r})
$$

with the notation and values given in [30]. Again, the shift is measured at the atomic frequency $(102.5 \mathrm{~nm})$. The intensity is meant to correspond to the energy flow of laser power in one direction only - hence, the factor of two in the above equation. The coefficients $\beta_{A C}$ have been computed for a linearly polarized continuous-wave laser field. They can be used without modification for our purpose, as the $\sigma_{+} / \sigma_{-}$ counter-propagating laser beams produce a linear polarization everywhere. The linear polarization direction forms a helix in space. This helix alternates between two configurations as $\sigma_{+} / \sigma_{-}$pulses alternate their directions every second pulse collision event. Despite this complication the laser polarization that the atoms see is linear at each instant of time [63]. Moreover the AC-Stark shift of two-photon direct frequency comb spectroscopy is determined by the time-averaged intensity, not the much larger peak intensity of the corresponding pulse train [43]. Therefore, $I(\vec{r})$ in (5.13) refers to the time-averaged intensity that is readily measured with a laser powermeter. Averaging the laser intensity radially, we obtain an estimation for the AC-Stark shift of

$$
\langle I\rangle=\frac{2 P / T_{2}}{\pi w_{0}^{2}}
$$

where $w_{0}=80 \mu \mathrm{m}$ is the laser waist radius, $P=P_{L} T_{2}$ is the time-averaged laser power measured after the $205 \mathrm{~nm}$ enhancement cavity (see "PM" in Fig. 3.1) and $T_{2}=5.4 \times 10^{-4}$ is the transmission of the back reflecting mirror (see section 3.4). With these numbers we obtain an approximate AC-Stark coefficient of $\kappa_{A C}=47 \mathrm{~Hz} / \mu \mathrm{W}$ from (5.13) and (5.14). From a Monte Carlo simulation, that 
takes the real distribution of trajectories into account rather than using the averaged intensity of (5.14), we obtain a much more accurate value of $\kappa_{A C}=33 \mathrm{~Hz} / \mu \mathrm{W}$.

However, this value is more than four times smaller than the AC-Stark shift coefficient that is obtained experimentally and presented in table 4.1. This discrepancy is significantly larger than the expected uncertainties of the measured laser power, the back reflecting mirror transmission, and waist size combined. We could not identify the reason for this discrepancy. However, it should be noted that this serves as a cross check only, and the data evaluation only assumes a linear AC-Stark shift with any $\kappa_{A C}$. With the measured laser power $\langle P\rangle=33 \mu \mathrm{W}$ averaged over all valid data, we get $\left\langle\delta \nu_{A C}\right\rangle \approx 4.6 \mathrm{kHz}$ with the larger, experimental AC-Stark shift coefficient.

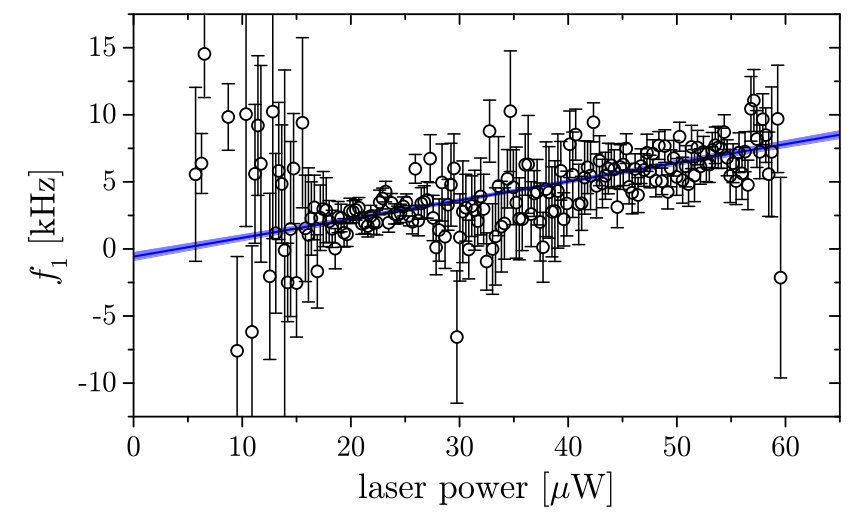

Figure 5.7.: AC-Stark shift (measured at the atomic frequency relative to the final result of this work) as a function of the laser power measured after the $205 \mathrm{~nm}$ enhancement cavity. To generate this graph, each line scan has been corrected for the CIFODS $\left(\kappa_{D S}\right)$, the second-order Doppler shift $\left(\kappa_{S O D}\right)$ and the pressure shift $\left(\kappa_{P S}\right)$ using the results of the global minimization in table 4.1. The linear function is the result of the global minimization with $\kappa_{A C}=139.7(9.1) \mathrm{Hz} / \mu \mathrm{W}$ taken from the same table and shown together with the $1 \sigma$ confidence interval bands. For clarity the data has been binned on the horizontal axis (bin size $0.3 \mu \mathrm{W}$ ) but not for computing the global minimization. The error bars are obtained from a weighted average of lines scans within each bin assuming these line scans are subject to shot noise only. The intercept at zero laser power is off by $-0.57 \mathrm{kHz}$ from the final result due to the remaining corrections listed as "total corrections" in table 5.1.

We emphasize that these considerations are only consistency checks to verify the order of magnitude of the AC-Stark shift. None of them are used in the global fitting procedure of section 4.4. The only assumption there is the linearity of the laser power measurement. For this we have verified that the transmission of the back reflecting mirror varies only at the level of few percent with laser spot position. The angular dependence of the powermeter is less than $1 \%$ at incidence angles smaller than $5^{\circ}$. The nonlinearity in the $100 \mu \mathrm{W}$ range is below 0.5\%. By moving the powermeter, we could not find any significant position dependence. The calibration uncertainty of the powermeter is specified to be less than $7 \%$. However this is not a requirement since we extrapolate to zero laser power. For visualization of the magnitude of the 
AC-Stark shift in our experiment, we need to separate it from the other corrections. For this purpose we correct all line scans for all shifts except for the AC-Stark shift using the results of the global minimization - as was also done for the CIFODS. Figure 5.7 shows the result.

We also considered the possibility of a higher-order and hence nonlinear contribution to the AC-Stark shift. The experimental data shown in Fig. 5.7 does not exclude this possibility. In perturbation theory, the AC-Stark shift has only even orders. The largest close-to-resonant contribution is due to the two-photon coupling of the $3 \mathrm{~S}$ and $3 \mathrm{D}$ states. This effect can take place via the excitation paths $3 \mathrm{~S} \rightarrow$ $n \mathrm{P} \rightarrow 3 \mathrm{D} \rightarrow n^{\prime} \mathrm{P} \rightarrow 3 \mathrm{~S}$ with intermediate states $n \mathrm{P}, 3 \mathrm{D}$ and $n^{\prime} \mathrm{P}$, where $n$ and $n^{\prime}$ are summed over all bound and continuum states. For these paths, we derive the frequency shift with fourth-order perturbation theory given by

$$
\delta \nu_{A C}^{(4)} \approx \frac{\left(\omega_{r} \tau\right)^{2}}{2}\left(\frac{e E_{0}}{6 \hbar}\right)^{4}\left(S_{+}-S_{-}\right)^{2} \sum_{\substack{D \\ n_{1}, n_{2}}} \frac{e^{-\left(n_{1}^{2}+n_{2}^{2}\right)\left(\omega_{r} \tau / 2\right)^{2}}}{\omega_{3 \mathrm{D}, 3 \mathrm{~S}}-\left(n_{1}-n_{2}\right) \omega_{r}}
$$

where

$$
S_{ \pm} \equiv \sum_{n} \frac{\mu_{3 \mathrm{~S}, n \mathrm{P}} \mu_{n \mathrm{P}, 3 \mathrm{D}}}{\omega_{n \mathrm{P}, 3 \mathrm{~S}} \pm \omega_{c}}
$$

Here, the $\mu$ are the corresponding dipole matrix elements (see [57]), $\omega_{c}$ is the comb carrier frequency, $\omega_{r}$ is the repetition rate, and the other labeled $\omega$ are the corresponding transition frequencies. While there are no resonances in (5.16), there are close-to-resonant contributions in (5.15) because our frequency comb is wider than the 3S-3D splitting. The sum extends over the 3D levels (fine and hyperfine) and two mode numbers of the frequency comb $n_{1}$ and $n_{2}$. With the mode spacing $\omega_{r}=2 \pi \times 315.2 \mathrm{MHz}$, we find that no mode combination are closer than 7 times the natural line width of the 3D level $(10.3 \mathrm{MHz})$, so we do not include an imaginary damping constant. By evaluating (5.15) with a peak field of $E_{0}=2.6 \mathrm{MV} / \mathrm{m}^{2}$, which corresponds to the mean experimental conditions, we find that this shift is much less than $1 \mathrm{~Hz}$ [63], and thus negligible.

The $2.4 \mathrm{GHz}$ discharge that is used for dissociating hydrogen molecules into atoms (see section 3.4) might also give rise to a Stark shift. The discharge is located outside the vacuum system (see Fig. 3.6 and Fig. 3.7) and is therefore shielded by the vacuum vessel. An additional shield is provided by the fiber mount of the DF detector assembly that also contains the compensation electrodes (see Fig. 5.14). The microwave electric field strength $E_{\mathrm{RF}}$ at the position of the atoms has been estimated to be around $0.3 \mathrm{~V} / \mathrm{m}$ in a similar double shielded apparatus [64]. The dominant contribution to this AC-Stark shift is due the coupling of the 3S state to the $3 \mathrm{P}_{3 / 2}$ with a level separation of $2.93 \mathrm{GHz}$ is given by

$$
\delta \nu_{A C}^{\mathrm{RF}}=\left(\frac{\mu_{3 \mathrm{~S}, 3 \mathrm{P}} E_{\mathrm{RF}}}{2 h}\right)^{2} \frac{1}{2.93 \mathrm{GHz} \pm 2.4 \mathrm{GHz}}
$$


where the counter-rotating terms with the two signs have to be added. Assuming linear polarization and $\mu_{3 \mathrm{~S}, 3 \mathrm{P}}=6 e a_{0}$ for the $3 \mathrm{~S}(F=1, M=0) \rightarrow 3 \mathrm{P}_{3 / 2}(F=2, M=0)$ component we obtain $\delta \nu_{A C}^{\mathrm{RF}}=1.2 \mathrm{~Hz}$.

Another source for an AC-Stark shift is the black body radiation. The room temperature $(T=300 \mathrm{~K})$ black body shift of the 1S-3S centroid has been computed to $-9.06 \mathrm{~Hz}$ [65]. This serves as a upper limit as parts of the structure surrounding the PCV are certainly below room temperature and this shift simply scales with $\propto T^{4}$ according to the Stephan-Boltzmann law. Since we are measuring only one hyperfine component we also need to take the black body shift of the hyperfine splitting into account. For the ground state this shift is in the $\mathrm{nHz}$ range and assumed to be of the same order for the $3 \mathrm{~S}$ state [66].

It is conceivable that ionisation and photoelectrons generated by the scattered laser light can give rise to a laser power dependent DC-Stark shift and mimic a contribution to the AC-Stark shift. The stray field detection and compensation method detection 5.5 would be sensitive to this effect. However, we do observe only a negligible DC-Stark shift with this method (see section 5.5).

\subsection{Second-order Doppler shift}

For the second-order Doppler shift, as with the other systematic shifts, we first give a theoretical model to derive some properties and to estimate the expected magnitude but then use the data to extract the systematic shifts. The second-order Doppler (SOD) shift of atoms at the velocity $v$ is given by $-0.5(v / c)^{2} \nu_{0}$, with the atomic frequency $\nu_{0}$. The simplest assumption is a thermal beam of $1 \mathrm{~S}$ atoms of mass $m$ with a Maxwellian velocity distribution of $p(v) \sim v^{2} e^{-\left(v / v_{0}\right)^{2}}$ with the most probable velocity of $v_{0}=\sqrt{2 k_{B} T / m}$. The number of contributing atoms per second is then given by the flux, i.e. by $p(v) \sim v^{3} e^{-\left(v / v_{0}\right)^{2}}$ [61], that is the number of atoms per unit time that pass through the pulse collision volume (PCV) with a velocity $v$. To estimate the expected signal, we need to take the velocity-dependent excitation probability into account. There are two limiting regimes. Atoms that remain within the PCV longer than the lifetime of the upper state reach steady state. Therefore the signal is proportional to the travel time through the PCV, i.e. $\sim 1 / v$ (see also section 3.5). The "steady state" normalized weighting function and the mean quadratic velocity is given by:

$$
w^{(s s)}(v)=\frac{4}{\sqrt{\pi} v_{0}^{3}} v^{2} e^{-\left(v / v_{0}\right)^{2}} \Rightarrow \overline{v^{2}}=(3 / 2) v_{0}^{2} .
$$

In the other limiting case, the signal from atoms that travel through the PCV in a time that is much shorter than the upper state lifetime is described by coherent Rabi flopping. In our case the Rabi frequency is around $2 \pi \times 240 \mathrm{~Hz}$ with (5.14), the mean laser power given in section 5.2, and the coefficients given in [30]. The length of the $\mathrm{PCV}$ is $c T_{1 / 2}=0.60 \mathrm{~mm}$, where the FWHM of the light intensity matters here, since 
the two-photon Rabi frequency is proportional to intensity. Therefore, it is safe to assume that none of the atoms get close to performing a full Rabi cycle and we can expand the Rabi flopping to yield an excitation probability that is proportional to the square of the travel time through the PCV, i.e. $\sim 1 / v^{2}$. Hence, the normalized "non-stationary" weighting function for this limiting case is given by:

$$
w^{(n s)}(v)=\frac{2}{v_{0}^{2}} v e^{-\left(v / v_{0}\right)^{2}} \quad \Rightarrow \quad \overline{v^{2}}=v_{0}^{2} .
$$

From these two weighting functions we obtain the SOD shift coefficients as $\kappa_{S O D}^{(s s)}=$ $-3 k_{B} \nu_{0} / 2 m c^{2}=-403 \mathrm{~Hz} / \mathrm{K}$ and $\kappa_{S O D}^{(n s)}=-k_{B} \nu_{0} / m c^{2}=-268 \mathrm{~Hz} / \mathrm{K}$ with $w^{(s s)}(v)$ and $w^{(n s)}(v)$ respectively.

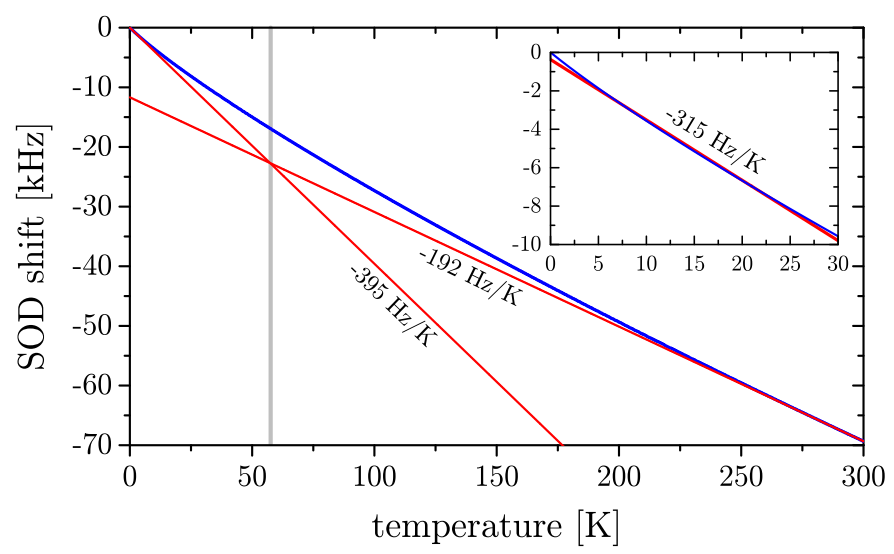

Figure 5.8.: Blue: Modelling the second-order Doppler (SOD) shift with a Monte Carlo simulation without the AC-Stark and the pressure shift relative to the unperturbed transition frequency as a function of temperature assuming a Maxwellian flux distribution from a nozzle of $p(v) \sim v^{3} e^{-\left(v / v_{0}\right)^{2}}$. The shift is measured at the atomic frequency. Two slopes are present with a crossover temperature/velocity $(T \approx 60 \mathrm{~K}, v \approx 1200 \mathrm{~m} / \mathrm{s})$ determined from the intersection of the tangents at $T=0$ and $T=300 \mathrm{~K}$ (red). The inset magnifies the experimentally evaluated temperature region with a straight line fitted to the temperature values of $T=4.5 \mathrm{~K}, 7 \mathrm{~K}, 15 \mathrm{~K}$ and $30 \mathrm{~K}$ only (red), that are assigned with the error bars shown in Fig. 5.10 and Fig. 5.10. The slope agrees very well with the one derived from the experimental data and the intercept of $-0.37 \mathrm{kHz}$ may be used as another way to quantify the nonlinear contribution to the SOD shift (see table 5.1).

Assuming a pure Maxwellian flux distribution, we conducted Monte Carlo simulations (see section 2.5) that goes beyond the two limiting cases. The results of these simulations are evaluated exactly like the experimental data - that is with the same fitting function (4.1) and the same line sampling grid (see section 4.1). The resulting SOD shift as a function of temperature is shown in Fig. 5.8. By fitting a straight line to the simulated data at the four evaluated temperatures $(T=4.5 \mathrm{~K}, 7 \mathrm{~K}, 15 \mathrm{~K}$, $30 \mathrm{~K}$ ) along with the experimental uncertainties, we get $\kappa_{S O D}=-311 \mathrm{~Hz} / \mathrm{K}$. This 
value agrees very well with the one derived from the experimental data with the global minimization of (4.4): $\kappa_{S O D}=-317(25) \mathrm{Hz} / \mathrm{K}$ (see table 4.1) which assumes a linear dependence for $T \leq 30 \mathrm{~K}$. The larger temperature data is used to check the model assumptions only. Comparing with the simulations, we obtain an estimate for a correction that is due to the nonlinearity of the SOD shift.

The main problem for the model is not so much the inaccuracy of the Monte Carlo simulations, but more the assumptions about the experimental conditions. In particular, the assumed velocity distribution may or may not be described by a Maxwellian. Non-equilibrium processes, recombination, and temperature gradients within the nozzle, as well as collisions within the beam, may lead to deviations from the ideal distribution. These deviations are difficult to determine experimentally. To shed more light on these limitations, we also ran Monte Carlo simulations for a class of velocity distributions of the form $p(v) \propto v^{q} e^{-\left(v / v_{0}\right)^{2}}$ with exponents $q=2.0,2.6$, $3.0,3.3,3.6$ and 4.0 with a temperature range of $T=1 \ldots 300 \mathrm{~K}$, with $q=3$ being the expected distribution for an effusive beam from a nozzle [61]. As one can see in Fig. 5.9, the model predictions spread with larger temperatures but agree better at lower temperatures. We vary the exponent $q$ as well as the transition frequency to match the experimental data for $T \leq 30 \mathrm{~K}$. By interpolating the exponent $q$ between the discrete simulations we find that $q=3.02(51)$ has the best agreement. This suggests that the true velocity distribution is close to a Maxwellian, at least for the evaluated low temperatures.

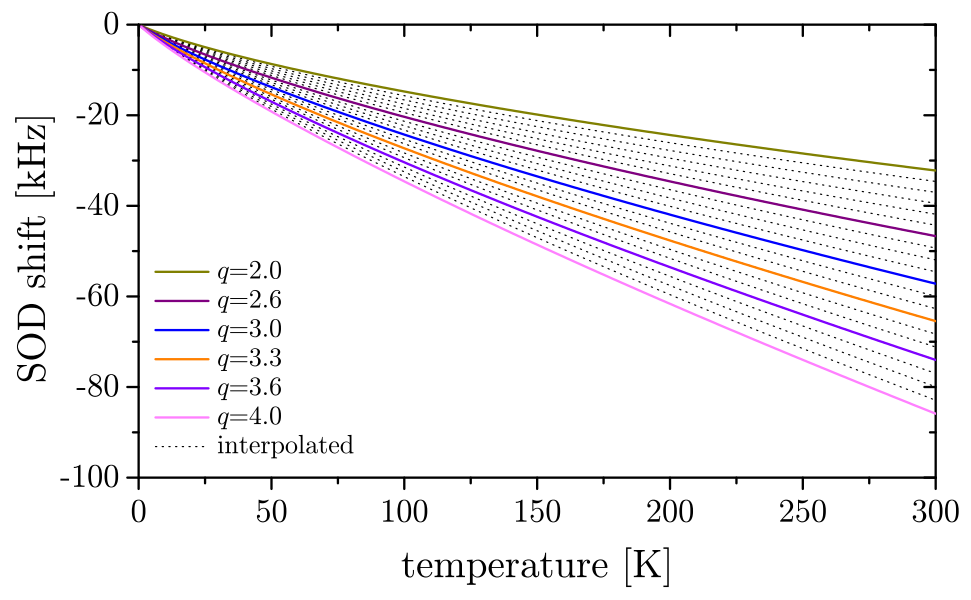

Figure 5.9.: Simulated second-order Doppler shift (measured at the atomic frequency) as a function of the temperature for different velocity distributions of the form $v(p) \propto$ $v^{q} e^{-\left(v / v_{0}\right)^{2}}$. The dashed curves are interpolated between the actual computed ones shown with solid curves.

This can be compared with a recent publication [62], which investigated the velocity distribution of a cryogenic hydrogen beam using a similar nozzle design to the one used here. In that publication, the distribution was found to significantly deviate from a Maxwellian $(q \approx 5)$ in contrast to the results here. However, the measure- 
ments in [62] were also measured far away from the nozzle with a small divergence angle so that it was likely only atom/atom or atom/molecule collisions within the nozzle could account for the on-axis beam. Further, the main conclusion of [62] was that one should expect a distribution close to a Maxwellian provided a few modest conditions are fulfilled - for instance, there should be a direct line of sight from a cold inner surface of the nozzle to the PCV. That is the case here since the PCV is very close to the nozzle (see the discussion in section 5.4). Therefore, based on the conclusions of [62], we believe a Maxwellian (i.e. $q=3$ ) is expected.
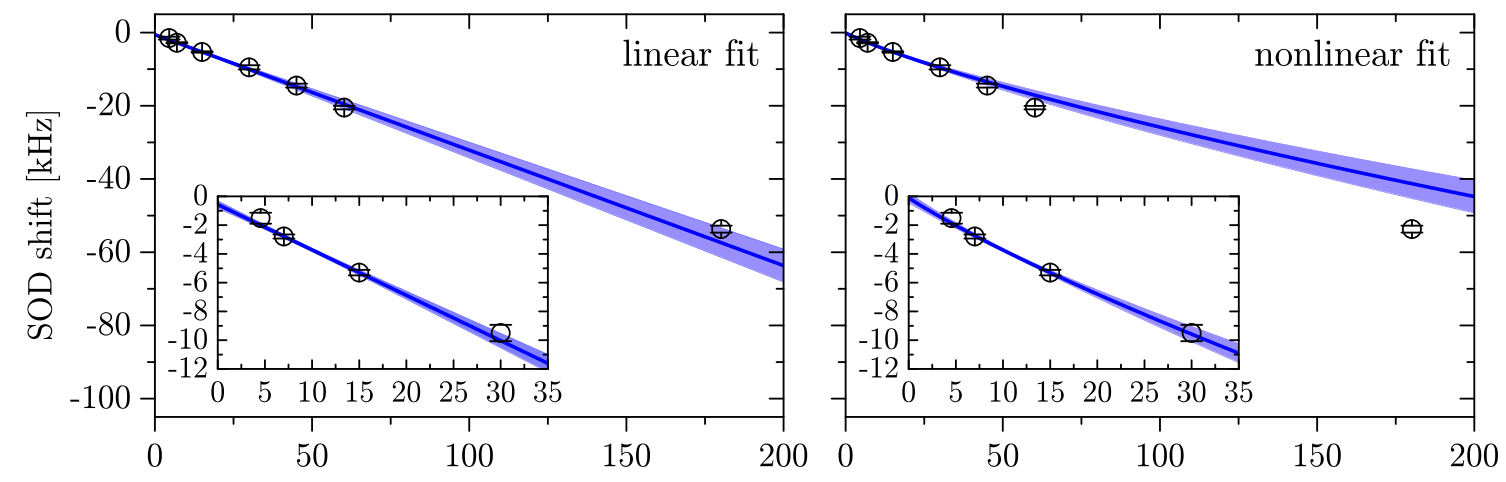

temperature $[\mathrm{K}]$

Figure 5.10.: Second-order Doppler (SOD) shift of the experimental data (black circles) (measured at the atomic frequency relative to the final result of this work) after correcting each line scan for the CIFODS $\left(\kappa_{D S}\right)$, the AC-Stark $\left(\kappa_{A C}\right)$ and the pressure shift $\left(\kappa_{P S}\right)$ using the results of the global fit in table 4.1. The data for the same nozzle temperatures has been binned. The error bars are obtained by assuming detection shot noise and the usual error propagation with $\chi^{2}$-scaling (see section 4.2). Left: Fitting a linear function (blue) to the $T \leq 30 \mathrm{~K}$ data only, also "predicts" the larger temperature data $\left(\chi^{2} /\right.$ dof $\left.=1.10\right)$. The inset shows the evaluated data magnified. The $T=0$ extrapolates to $-0.58 \mathrm{kHz}$ which agrees well with the "total corrections" $-0.57 \mathrm{kHz}$ in table 5.1). The light blue region is the $1 \sigma$ confidence interval of the fit. Right: The same data fitted with a nonlinear model obtained from a Monte Carlo simulation (in blue) that assumes a velocity distributions for the atoms of $p(v) \propto v^{q} e^{-v^{2} / v_{0}^{2}}$ with $q=3.16(18)$. Again the larger temperature data $(T>30 \mathrm{~K})$ has not been used for the fit $\left(\chi^{2} /\right.$ dof $\left.=0.51\right)$, i.e. to find the optimum exponent. In this case, the higher temperature data is not predicted as well as with the linear fit. This suggests that the true velocity distribution is not exactly within the class of distributions used in Fig. 5.9. The Monte Carlo model and the linear model disagree by $0.46 \mathrm{kHz}$ at $T=0$, but the uncertainty due to the nonlinear SOD contributions are taken into account as described in the text.

To visualize the magnitude of the SOD shift and to further investigate the limitations of the linearity assumption, we use the parameters from the global fit (table 4.1) to correct for all other shifts (CIFODS, AC-Stark, pressure shift) and plot the remaining dependence on the SOD in Fig. 5.10. The data is binned for the experimental temperatures $T=4.5 \mathrm{~K}, 7 \mathrm{~K}, 15 \mathrm{~K}, 30 \mathrm{~K}, 45 \mathrm{~K}, 60 \mathrm{~K}$ and $180 \mathrm{~K}$. The 
larger temperatures $(T>30 \mathrm{~K})$, that have not been used in the global minimization, are nevertheless corrected with the same parameters. The linear model fits the low temperature data very well and even describes the larger temperatures within the confidence bands. In contrast, using the Monte Carlo simulations with $q=3.16(18)$ obtained by using the temperatures $T \leq 30 \mathrm{~K}$ does not "predict" the larger temperature behaviour as well as the simple linear model. This reveals a limitation of the model. As a consequence, we apply the linearity assumption but quadratically add the full range of simulations, i.e. the difference between the $q=2$ and $q=4$ models to the experimental uncertainties $\sigma_{1, i}$ (but not to $\sigma_{2, i}$ and $\sigma_{3, i}$; see section 4.4). In this way we also put more weight on the low temperature data, increase the error bar for the less certain larger temperatures, and ignore the largest temperatures. The error bars shown in Fig. 5.10 are not enlarged in this way. At a temperature of $7 \mathrm{~K}$ the SOD shift requires a correction of $+2.2 \mathrm{kHz}(+3.2 \mathrm{kHz}$ for all data, see table 5.1). We emphasize that we need to understand this correction only to within say $10 \%$ in order to prevent it from contributing significantly to our final error budget. From the linear SOD extrapolation we get an uncertainty of $0.26 \mathrm{kHz}$ as described in section 5.12 and listed in the error budget shown in table 5.1.

\subsection{Pressure shift}

The hydrogen atoms in the beam collide with other hydrogen atoms in the $1 \mathrm{~S}$ and 3S state as well as with hydrogen molecules that have not been dissociated or have recombined inside the nozzle. All of them give rise to different collisional shifts (i.e. pressure shifts), and all can be taken as being proportional to the number of collisions per second, which is in turn proportional to the density or flux of atoms/molecules. In order to extrapolate the pressure shift from intra-beam collisions to zero, we changed the distance between the PCV center and the hydrogen nozzle by translating the nozzle along the laser beam. Two distances, $d_{1}=19.1 \mathrm{~mm}$ and $d_{2}=27.1 \mathrm{~mm}$, have been used for the current data set. We assume the pressure shift is proportional to the density of colliding atoms and undissociated molecules. The density of atoms and molecules from the beam is expected to reduce as the square of the distance from the source. We do not rely on this assumption, but instead calibrate the intra-beam density from the line amplitudes (see below).

As a consistency check, we have tried to locate the source point by extrapolating the observed line amplitudes. Atoms and molecules that escape the nozzle have either collided with other atoms or molecules inside the nozzle, or have emerged from the inner surface of the nozzle. Since the PCV is close to the nozzle, there is a direct line of sight to the inner wall of the back orifice. Unintentionally, the diameter of the back orifice was made $200 \mu \mathrm{m}$ smaller than the front orifice $(1.3 \mathrm{~mm}$ instead of $1.5 \mathrm{~mm}$ ) as we found out after the measurement, thereby increasing the visible inner surface. To better locate the source point and to get a good estimate of the local flux of atoms within the beam, we mounted the fibers that detect the Doppler-broadened 


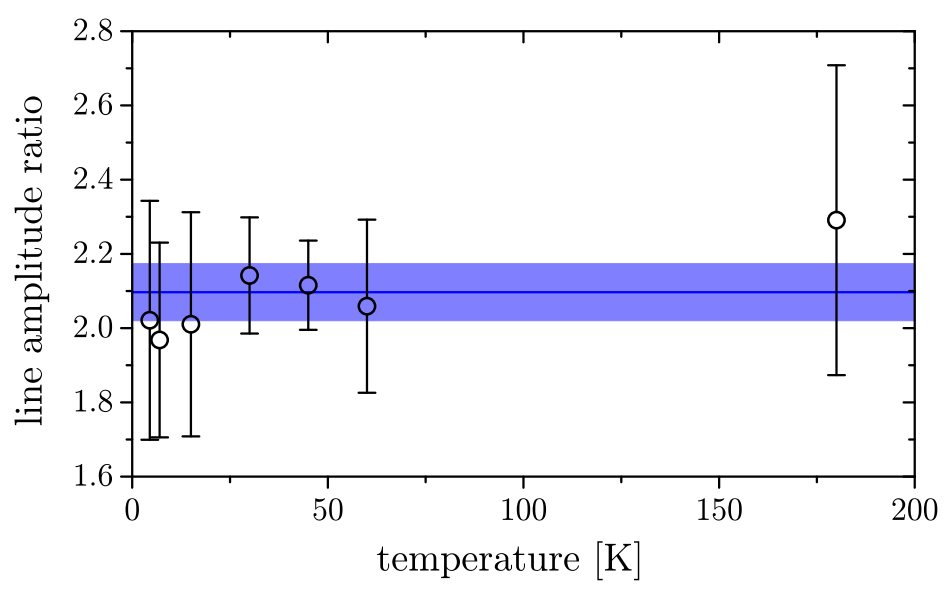

Figure 5.11.: Ratio of line amplitudes $A_{i}$ at $d_{1}=19.1 \mathrm{~mm}$ and $d_{2}=27.1 \mathrm{~mm}$ distance between the PCV center and the hydrogen nozzle as a function of temperature (blue circles). The ratio is temperature independent with an overall average of $2.10(8)$ (blue line with $1 \sigma$ confidence interval). This agrees well with the expectations $(27.1 \mathrm{~mm} / 19.1 \mathrm{~mm})^{2}=2.01$, if the atoms emerge from the rear inner wall of the nozzle and in strong disagreement with the assumption that atoms are emerging from the front orifice of the nozzle $(17.1 \mathrm{~mm} / 9.1 \mathrm{~mm}=3.53)$. The error bars shown here are the standard deviations of the data for the given temperature, because the uncertainty due to shot noise is much smaller. The stability of this ratio over the course of the whole measurement period gives us confidence that the long term average atomic flux is also stable.

(DB) normalization signal (see section 3.6) and the nozzle on the same translation stage. With this, the normalization is unchanged as the nozzle is moved, and the scaled line amplitudes $A_{i}$ in (4.1) and (4.3) are a good measure for the flux through the PCV. The average line amplitude ratio for the two distances was determined to be 2.10(8), without a noticeable dependence on temperature. This agrees well with $(27.1 \mathrm{~mm} / 19.1 \mathrm{~mm})^{2}=2.01$, if the distance is measured from the rear inner wall of the nozzle. If the atoms would emerge from the front orifice of the nozzle (nozzle length $10 \mathrm{~mm})$, the amplitude drop would be $(17.1 \mathrm{~mm} / 9.1 \mathrm{~mm})^{2}=3.53$. Figure 5.11 shows the average line amplitude ratios for different temperatures.

With the global minimization of (4.4) we obtain the pressure shift coefficient to be $\kappa_{P S}=0.58(22) \mathrm{kHz}$ (see table 4.1). To visualize the magnitude of the pressure shift we again use the parameters from the global fit (table 4.1) to correct for all other shifts (CIFODS, AC-Stark and second-order Doppler) and plot the remaining dependence on pressure in Fig. 5.12. The histogram at the bottom reveals two peaks that correspond to the nominal distances $d_{1}=19.1 \mathrm{~mm}$ and $d_{2}=27.1 \mathrm{~mm}$ measured from the rear inner wall of the nozzle. The significant widths of these peaks are not due to fluctuation of the laser power or atomic flux, because those have been taken out by the normalization (see section 3.6). Instead we believe that these fluctuations are real changes of the distance between the PCV center and the source point that 
add to the nominal distances that we adjusted the translation stage to. The cryostat that holds the nozzle sits on a $50 \mathrm{~cm}$ long lever arm that reaches into the vacuum chamber with a bellow. As ice layers form on the outside of the bellow, the structure deforms so that the PCV to nozzle distance may change by up to 1-2 $\mathrm{mm}$. This can easily explain the observed width of the peaks in the lower part of Fig. 5.12. Assuming that these fluctuations are real, this is yet another motivation to use the normalized amplitudes, $A_{i}$, instead of the measured PCV-to-nozzle distances to extrapolate the pressure shift. An additional source of noise might be variations of the degree of dissociation within the beam.

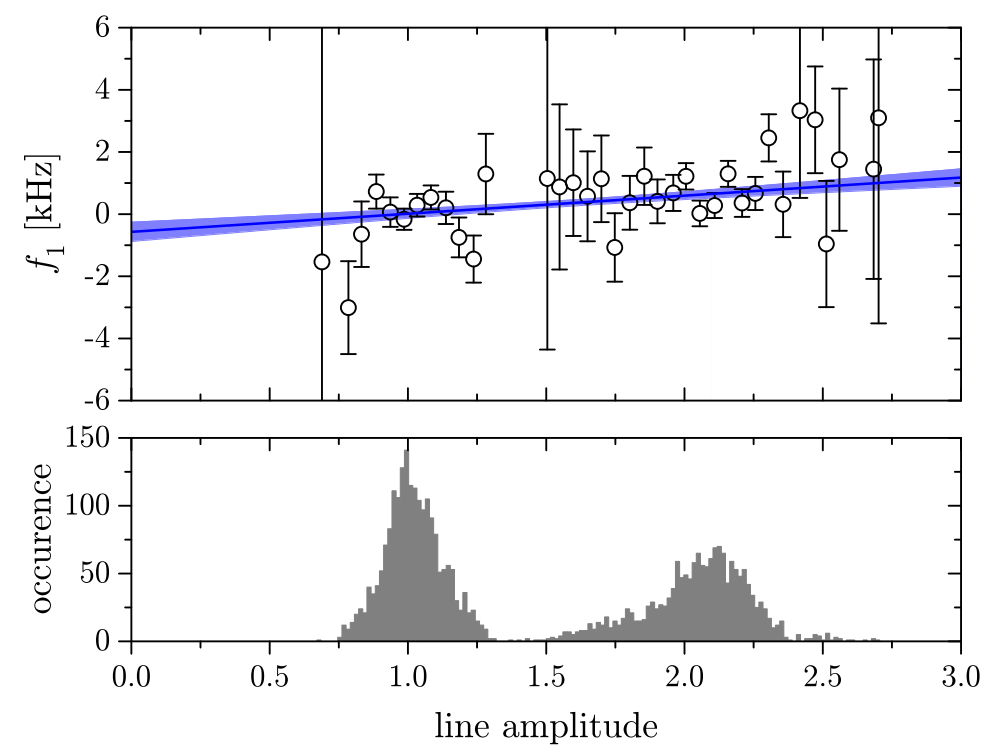

Figure 5.12.: Top: Intra-beam pressure shift (measured at the atomic frequency relative to the final result of this work) determined by correcting each line scan for the CIFODS $\left(\kappa_{D S}\right)$, the second-order Doppler $\left(\kappa_{S O D}\right)$, and the AC-Stark $\left(\kappa_{A C}\right)$ shifts using the results of the global fit in table 4.1. The horizontal axis are the normalized and scaled line amplitudes $A_{i}$. This amplitude is proportional to the atomic flux and hence to the pressure shift. The linear function is the result of the global minimization with $\kappa_{P S}=0.58(22) \mathrm{kHz}$ taken from table 4.1 and shown together with the $1 \sigma$ confidence interval bands. The data is binned for clarity for the plot but not for the global minimization. The error bars are obtained from a weighted average of lines scans within each bin assuming these line scans are subject to shot noise only. The intercept at zero pressure differs by $-0.57 \mathrm{kHz}$ from the final result due to the remaining corrections listed as "total corrections" in table 5.1. At the bottom of the figure a histogram of the line amplitudes is shown. The two peaks correspond to the two distances between the PCV and the nozzle used in the experiment. The fitted line with confidence intervals in the upper part (blue) is mainly determined from the data in these peaks. The significant broadening is believed to be due to limitations of the reproducibility of this distance due to deformations of the structure when cooling. This effect may change the geometry by $1-2 \mathrm{~mm}$.

While the intra-beam collisional shift can be extrapolated, we could not determine 
the collisional shift from the background gas experimentally. The pressure is measured to be around $10^{-5}$ mbar with an ionization gauge placed in the central section of the vacuum chamber where the spectroscopy takes place. To estimate the background pressure shift, we are using a simple theoretical model based on the so-called impact approximation [67], which assumes long-range interactions between colliding species. The long-range van-der-Waals interaction between two hydrogen atoms was theoretically investigated in [68]. The interaction between hydrogen molecules and hydrogen atoms is more difficult to compute due to the complicated structure of the molecular levels. As an estimate, we assume that the van-der-Waals coefficient has the same order of magnitude. The physical reason for this choice is that the first dipole-allowed transition from the ground state of a hydrogen molecule is about $12.4 \mathrm{eV}$, which is close to the first dipole-allowed transition in atomic hydrogen of $10.2 \mathrm{eV}$. This allows us to treat the background gas as atomic hydrogen only.

Most of the measurements in this work have been done at a temperature of $7 \mathrm{~K}$, which is much smaller than the temperature of the background gas ( $\approx 300 \mathrm{~K})$. A model for the collisional shift experienced by a slow atomic beam that is emerged in a gas of faster moving atoms has been developed in [69] and is described by:

$$
\delta \nu_{P S}=\frac{1.29388}{2 \pi} n \xi_{\omega}^{(6)}\left(\frac{k_{B} T_{B}}{m_{B}}\right)^{3 / 10}
$$

where $n$ is a number density of the particles of the background gas, and $T_{B}$ is the temperature of the background gas with a particle mass of $m_{B}$. The coefficient $\xi_{\omega}^{(6)}=-4.325 \times 10^{-17} \mathrm{rad} \mathrm{m}^{2}(\mathrm{~m} / \mathrm{s})^{2 / 5}$ has been obtained in [69]. It is based on the calculation of the van-der-Waals interaction coefficients derived in [68]. With these numbers we obtain an estimate of the background gas pressure shift of about $-90 \mathrm{~Hz}$. Since our assumptions here are rather crude, we choose not to correct for this value but instead take its magnitude as an uncertainty (see table 5.1).

\subsection{DC-Stark shift}

The DC-Stark shift can be a significant problem for precision spectroscopy if stray electric fields are not properly compensated or shielded. In particular, at low temperatures a layer of frozen gases might build up at the surfaces of the nozzle. Free charges from ionized hydrogen and other photoionizations due to the excitation laser can build up on this layer and give rise to stray electric fields that can shift and broaden the line. This problem is aggravated in our apparatus because the pulse collision volume (PCV) is relatively close to the nozzle in order to obtain a large count rate. This is a disadvantage compared to the more traditional continuouswave excitation where the signal is generated far from non-conducting surfaces [7]. However, our compensation and shielding can be more effective in a small volume since, in practice, compensation works at one position in space only and otherwise 
relies on the homogeneity of that stray field. Obviously, this is is a much better assumption in a small excitation volume such as our PCV. Without proper shielding, we observe an increasing DC-Stark shift, along with broadening and quenching of the excited state such that the line essentially vanishes within minutes as the nozzle charges up. This behaviour is shown in Fig. 5.13.

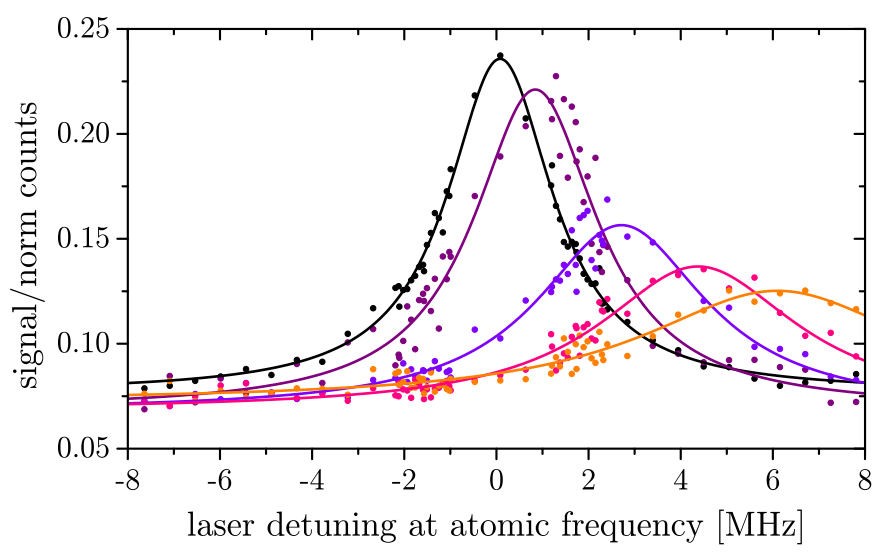

Figure 5.13.: A sequence of line scans recorded within a few minutes without the proper shielding of the pulse collision volume, where the excitation takes place. As non-conductive layers of frozen gas on the nozzle charge up by photoelectrons, the lines experience an increasing DC-Stark sift.

The DC-Stark shift can be computed analytically with second-order perturbation theory, or more accurately by diagonalizing the DC-Stark Hamiltonian. By diagonalizing the Hamiltonian for fields between 0 and $10 \mathrm{~V} / \mathrm{m}$ along the $z$-axis, and fitting a quadratic function to the level shift, we find $6.6224 \mathrm{~Hz} /(\mathrm{V} / \mathrm{m})^{2}$ for the $3 \mathrm{~S}(F=1, M=0)$ level, and $7.0890 \mathrm{~Hz} /(\mathrm{V} / \mathrm{m})^{2}$ for the $3 \mathrm{~S}(F=1, M= \pm 1)$ levels. Since the $1 \mathrm{~S}$ level has no close by $\mathrm{P}$ level, its DC-Stark shift is very small and can be neglected. The average DC-Stark shift of the $1 \mathrm{~S}(F=1)-3 \mathrm{~S}(F=1) \Delta M=0$ transitions is then given by $\alpha=6.9335 \mathrm{~Hz} /(\mathrm{V} / \mathrm{m})^{2}$ (all three components have the same strength [42]). To determine the DC-Stark line shift from the DC-Stark level shifts, we ran Monte Carlo simulations and evaluated the generated lines in exactly the same manner as the experimental data. In this way, we obtain $\alpha=6.8982 \mathrm{~Hz} /(\mathrm{V} / \mathrm{m})^{2}-$ effectively the same value for the purpose of this work (see below). Experimentally, any non-conductive surface such as layers of frozen gases, or any insulator, would charge up to a few Volts from the $\sim 10 \mathrm{eV}$ photon energies involved. With the typical cm-scale distances inside the apparatus, MHz-scale line shifts could be expected, if no appropriate shielding was established. DC-Stark shifts at the $\mathrm{MHz}$ level as can be seen in Fig. 5.13. Therefore, proper shielding and/or compensation is essential.

To measure and compensate the stray electric fields in all directions, we use three pairs of electrodes around the PCV (shown schematically in Fig. 3.9 and as a photograph in Fig. 5.14). The whole lens and electrode mount is made from aluminum and coated with a conductive graphite layer. With this setup, we can 


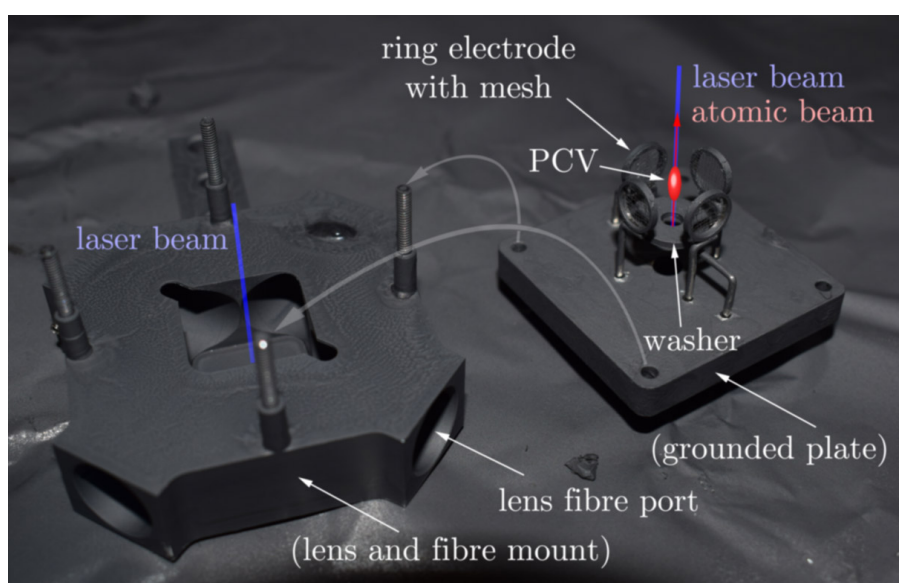

Figure 5.14.: Compensation electrodes and mount for the lenses and fibers used for signal collection. When mounted, the pulse collision volume (PCV) is located between the washer, lens, and fiber mount. The nozzle is located behind the grounded plate. The four ring electrodes with meshes (90\% transmission) serve as pairs of electrodes to apply and/or compensate fields perpendicular to the atomic beam. The meshes are important to shield the fields originating from the lenses that sit behind because they cannot be covered with conducting graphite like all other parts in this picture. The graphite coatings also prevent contact potentials.

apply three voltage differences individually. To measure the component of the stray field $E_{s}$ in one direction we subsequently apply three voltages $0,+6 \mathrm{~V}$ and $-6 \mathrm{~V}$ to a given pair of electrodes. This adds fields 0 and $E_{ \pm}$to the stray field such that we can observe a DC-Stark shift relative to an arbitrary reference frequency $f_{0}$ of:

$$
f_{ \pm}=f_{0}+\alpha\left(E_{ \pm}-E_{s}\right)^{2} .
$$

With three measured frequencies, we fit a parabola to the frequencies as a function of the applied voltage and determine the DC-Stark shift due to the stray electric field

$$
\delta \nu_{D C}=\alpha E_{s}^{2} \approx \frac{\left(f_{+}-f_{-}\right)^{2}}{8\left(f_{+}+f_{-}-2 f_{0}\right)}
$$

and the required compensation voltage. Note that for the latter we neither need to know the value of $\alpha$ nor do we need to know what field is generated per applied voltage. However, this knowledge serves as a cross check. This procedure is performed on a daily basis for all three directions.

In doing so, we have found that the cage formed by the compensation electrodes provides sufficient shielding so that we simply ground all electrodes during the measurement. This has the advantage of knowing the relative voltage $(=0 \mathrm{~V})$ more accurately. Nevertheless we verified that the stray fields are small at the beginning of each a measurement day. The measured stray fields in all three directions are presented in Fig. 5.15 and allow us to determine an average DC-Stark shift of 31(14) Hz (see table 5.1). 
In addition to the inhomogeneity of the stray fields, the compensation fields may also be inhomogeneous. We use a finite element analysis of the setup and found that the applied fields vary only by about $5 \%$ across the length of the PCV. This finding was confirmed by comparing with other compensation mounts.

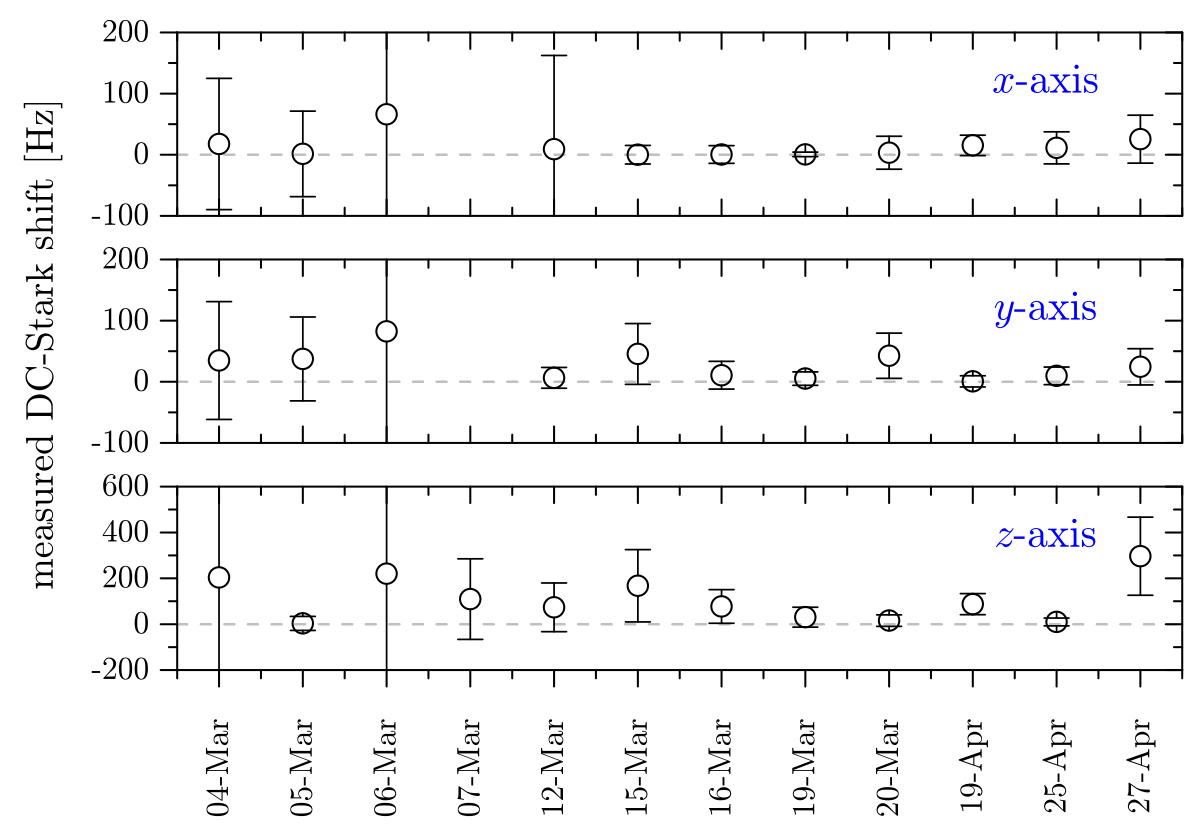

Figure 5.15.: DC-Stark shift measurement for almost all measurement days in 2018 (at the atomic frequency). The upper plot and middle plot show the stray field components perpendicular to the atomic beam ( $x$ and $y$-axis) measured through the corresponding DCStark shift by applying a bias field. The bottom plot shows the same data for the stray field component along the atomic beam ( $z$-axis). The weighted mean values are $2(3) \mathrm{Hz}$, $7(6) \mathrm{Hz}$ and $22(12) \mathrm{Hz}$ for stray fields in the $x, y$ and $z$-direction respectively (all with $\chi^{2} /$ dof $<1$ ). As expected, the stray field along the $z$-direction is the largest with around $1.8 \mathrm{~V} / \mathrm{m}$. The error bars have been obtained by the usual error propagation assuming shot noise for the detected signals and a rather unusual second order error propagation through (5.22), because the derivatives of this expression almost vanish in practice $\left(f_{+} \approx f_{-}\right)$in first-order.

An additional effect of stray electric fields is level mixing. In particular, the $3 \mathrm{P}$ level acquires a $3 \mathrm{~S}$ contribution that we can estimate by diagonalizing the $3 \mathrm{~S} / 3 \mathrm{P}$ manifold as described above. Taking the Lamb-shift into account, as in [57] eqn.(55.7), the perturbed $3 \mathrm{~S}$ wave function of the excited state is approximately given by

$$
|\psi\rangle \approx|3 \mathrm{P}\rangle+\frac{n \sqrt{n^{2}-1} m_{J} F}{L}|3 \mathrm{~S}\rangle \equiv|3 \mathrm{P}\rangle+X_{3} E|3 \mathrm{~S}\rangle
$$

with $F \equiv a_{0} e E$, the Bohr radius $a_{0}$, the field strength $E, n=3, m_{J}= \pm 1 / 2$ and the Lamb shift $L=h \times 315 \mathrm{MHz}$ (using the $3 \mathrm{~S}^{-3} \mathrm{P}_{1 / 2}$ splitting only) and hence 
$X_{3}=1.7 \times 10^{-4}(\mathrm{~m} / \mathrm{V})$. This mixing allows for $1 \mathrm{~S}-3 \mathrm{P}$ excitation with two-photons and a Rabi frequency of $\Omega_{1 \mathrm{~S}-3 \mathrm{P}}=X_{3} E \Omega_{1 \mathrm{~S}-3 \mathrm{~S}}=3.4 \times 10^{-4} \Omega_{1 \mathrm{~S}-3 \mathrm{~S}}$. The last number is obtained with the maximum field of $1.8 \mathrm{~V} / \mathrm{m}$ detected in the experiment (see Fig. 5.15). The relative line strength of the $1 \mathrm{~S}-3 \mathrm{P}$ line is given by the square of the ratio of the Rabi-frequencies and hence the extra line has a relative strength of $1.2 \times 10^{-7}$. It is folded into the spectrum with the frequency comb and has hyperfine components and a line width of $29.5 \mathrm{MHz}$ [57]. To get an upper limit of the line pulling effect, we generate artificial line scan data by adding the weak 1S-3P line to the fully allowed lines with various detunings and fit the result in the same manner as the experimental data. The maximum line pulling obtained in this way is $4 \mathrm{mHz}$.

Not only does the 3P state acquire a small 3S admixture, the 3S state also obtains a small $3 \mathrm{P}$ component through an external DC field, which leads to an additional line broadening. With the same formalism we obtain for the $3 \mathrm{~S}$ state:

$$
|\psi\rangle \approx|3 \mathrm{~S}\rangle+\frac{n \sqrt{n^{2}-1} m_{J} F}{L}|3 \mathrm{P}\rangle \equiv|3 \mathrm{~S}\rangle-X_{3} E|3 \mathrm{P}\rangle
$$

In addition to the $3 \mathrm{~S} \rightarrow 2 \mathrm{P}$ decay matrix element, we obtain an additional decay path through the $3 \mathrm{P} \rightarrow 2 \mathrm{~S}$ channel. Since the inverse lifetime $\tau_{3 \mathrm{~S}}$ is proportional to the matrix elements squared, we obtain:

$$
\frac{1}{\tau_{3 \mathrm{~S}}}=\frac{1}{\tau_{3 \mathrm{~S}-2 \mathrm{P}}}+\frac{X_{3}^{2} E^{2}}{\tau_{3 \mathrm{P}-2 \mathrm{~S}}}=\frac{1}{158.37 \mathrm{~ns}}+\frac{X_{3}^{2} E^{2}}{5.4 \mathrm{~ns}} .
$$

With $E=1.8 \mathrm{~V} / \mathrm{m}$ the line width increases by $X_{3}^{2} E^{2} /(2 \pi \times 5.4 \mathrm{~ns})=3.4 \mathrm{~Hz}$ only. We can also use the two-photon optical Bloch equations (see section 2.5) to verify these small effects. Numerically we obtain a line broadening of $0.72 \mathrm{~Hz} /(\mathrm{V} / \mathrm{m})^{2}$, which is comparable to the analytic treatment given above. With the full level scheme we could also verify the line pulling due to the 3S-3P excitation. However, the numerical simulation does not allow us to separate the discussed line pulling from the direct DC-Stark shift.

\subsection{Zeeman shift}

The Zeeman shift of a hydrogenic $\mathrm{S}(F, M)$ state with a nuclear spin $1 / 2$ is obtained in first approximation with the Breit-Rabi formula

$$
-\frac{A}{4}+M g_{N} \mu_{N} B \pm \frac{A}{2} \sqrt{1+2 M x(A)+x(A)^{2}}
$$

where the "+" sign belongs to the $F=1$ total angular momentum and the "- " sign to $F=0$. Further, $x(A) \equiv\left(g_{J} \mu_{B}-g_{N} \mu_{N}\right) B / A$, the external stray magnetic field is given by $B$, the electronic and nuclear $g$-factors are given by $g_{J}$ and $g_{N}$, the Bohr and nuclear magneton are given by $\mu_{B}$ and $\mu_{N}=\mu_{B} m_{e} / m_{p}$, and the hyperfine 
splitting is given by $A$. The latter is very well measured for the $1 \mathrm{~S}$ state, $A_{1 \mathrm{~S}}=$ $1420405751.7667(10) \mathrm{Hz}[78]$ and extrapolated to the excited states with $A_{3 \mathrm{~S}}=$ $52609473.2(3) \mathrm{Hz}$ [10]. With the circular $\sigma_{+} / \sigma_{-}$polarization, all three components of the $1 \mathrm{~S}(F=1, M) \rightarrow 3 \mathrm{~S}(F=1, M)$ compatible with the $\Delta M=0$ selection rule are excited with the same strength [42].

With (5.26), we obtain a Zeeman shift of the $F=1, M= \pm 1$ levels:

$$
\frac{A}{4} \pm\left(g_{J} \mu_{B}+\frac{1}{2} g_{N} \mu_{N}\right) B
$$

The Landé $g$-factors $g_{J}=2$, and the nuclear (proton) $g$-factor $g_{N}=2.7928473508(85)[4]$ are identical for the $1 \mathrm{~S}$ and the $3 \mathrm{~S}$ level so that the Zeeman shift is cancelled exactly for these components within this level of approximation. For the $F=1, M=0$ component, we can expand the level shift (5.26), and find for the shift of the transition frequency:

$$
\delta \nu_{Z e e}^{M=0} \approx \frac{A_{3 \mathrm{~S}}}{4} x\left(A_{3 \mathrm{~S}}\right)^{2}-\frac{A_{1 \mathrm{~S}}}{4} x\left(A_{1 \mathrm{~S}}\right)^{2}=\frac{35.802 \mathrm{kHz}}{\mathrm{Gauss}^{2}} B^{2} .
$$

In our setup we compensate the stray magnetic field in all three directions within the PCV using 3 pairs of Helmholtz coils. The residual fields have been measured several times during the measurement campaign and were found to be $14(15) \mathrm{mG}$. Thanks to the quadratic dependence, the corresponding Zeeman shift is only 7.0(8.0) Hz.

One may ask if possible imperfections that are not taken into account above for instance polarization imperfections - increase this shift. However, the $\Delta M=0$ selection rule holds for any polarization [42]. The physical reason is that the twophoton operator does not act on spins, and there is no orbital angular momentum exchanged in an S-S transition.

In addition, relativistic corrections requires one to multiply the $g$-factors in (5.27) with a state dependent factor, which is given in first-order for the electron spin as (see [57] eqn.(47.4)):

$$
1-\frac{\alpha^{2}}{3 n^{2}}
$$

resulting in a linear Zeeman shift of the $F=1, M= \pm 1$ transitions of:

$$
\delta \nu_{Z e e}^{M= \pm 1}= \pm \frac{8}{27} g_{J} \alpha^{2} \mu_{B} B= \pm \frac{44 \mathrm{~Hz}}{\text { Gauss }} B .
$$

Note that the anomalous $g$-factor part cancels and only the state dependent part is relevant for the Zeeman shift. Higher order terms and the corresponding correction to the nuclear movement are neglected here. Effectively this very small shift turns into a mere line broadening if both components $F=1, M=+1$ and $F=1, M=-1$ occur with equal strength.

Lastly, the Breit-Rabi formula (5.26) is obtained by dialogizing the four-level HFS Hamiltonian, ignoring all other states. For completeness we have also included 
the 3P and 3D levels and numerically diagonalized the HFS Hamiltonian resulting in a quadratic Zeeman shift of $35.403 \mathrm{kHz} /$ Gauss $^{2}$ for the $3 \mathrm{~S}(F=1, M=0)$ and $1.386 \mathrm{kHz} /$ Gauss $^{2}$ for the $1 \mathrm{~S}(F=1, M=0)$ state. Neglecting the $M= \pm 1$ levels, we obtain a mean Zeeman shift of the $1 \mathrm{~S}-3 \mathrm{~S}$ transition of $11.339 \mathrm{kHz} / \mathrm{Gauss}^{2}$, which corresponds to a Zeeman shift of only $2.2(2.6) \mathrm{Hz}$.

\subsection{Line distorting effects}

The line distortion due to the second-order Doppler (SOD) shift can be described (similar to the usual Voigt profile) as a convolution of the Lorentzian (4.1) with the velocity distribution. As an approximation for the latter, we may assume any of the two limiting weighting functions of section 5.3. Atoms that reach steady state are expected to contribute to the signal with the weighting function $w^{(s s)}(v)$ (see (5.18)). Together with the SOD shifted frequency $\nu_{0}^{\prime}=\left(1-0.5(v / c)^{2}\right) \nu_{0}$ we arrive at the line shape

$$
\mathscr{L}_{S O D}^{(s s)}\left(\nu_{0}, \gamma\right) \propto \int_{0}^{\infty} \frac{v^{2} e^{-v^{2} / v_{0}^{2}} d v}{\left(1-0.5(v / c)^{2}\right) \nu_{0}-\nu+i \gamma / 2} \propto \sqrt{z} e^{z} \operatorname{erfc}(\sqrt{z})-\frac{1}{\sqrt{\pi}},
$$

with $z \equiv\left(\nu-\nu_{0}+i \gamma / 2\right) / \gamma_{S O D}$ and $\gamma_{S O D}=0.5\left(v_{0} / c\right)^{2} \nu_{0}=T \times 268.42935 \mathrm{~Hz} / \mathrm{K}(\mathrm{a}$ kind of SOD line width). The integral may be expressed through the complementary error function, but it may be preferable to use a continued fraction expression for large $|z|$ (i.e. small SOD widths) for its evaluation [74]. For the purpose of this work, only the imaginary part of this line shape is required, but the dispersive part may be useful for other applications.

Similarly, atoms that do not reach steady state contribute to the signal with the weighting function $w^{(n s)}(v)$ so that we obtain:

$$
\mathscr{L}_{S O D}^{(n s)}\left(\nu_{0}, \gamma\right) \propto \int_{0}^{\infty} \frac{v e^{-v^{2} / v_{0}^{2}} d v}{\left(1-0.5(v / c)^{2}\right) \nu_{0}-\nu+i \gamma / 2} \propto-e^{z} \Gamma(0, z) .
$$

The integral may be expressed through the incomplete gamma function $\Gamma(0, z)$, or an exponential integral $E_{1}(z)$. Again, only the imaginary part is used here and a continued fraction expression may be better for numerical evaluation [74]. At $T=7 \mathrm{~K}$ the SOD line width is $\gamma_{S O D}=1.88 \mathrm{kHz}$, which is much smaller than the natural line width of $\gamma=1.0050 \mathrm{MHz}$. Therefore both of these line shapes are very close to a Lorentzian. Figure 5.16 shows some examples.

To quantify the apparent line shift associated with asymmetric line distortions, one must first decide how to measure this shift. Since distortions imply that different parts of the line shape experience different shifts, one may employ the shift of the maximum or the mean shift of the half-width points. It turns out that these two definitions differ by a factor of two to first-order [70] and both are useful for a theoretical discussion, but of limited use for noisy experimental data. Adapted 


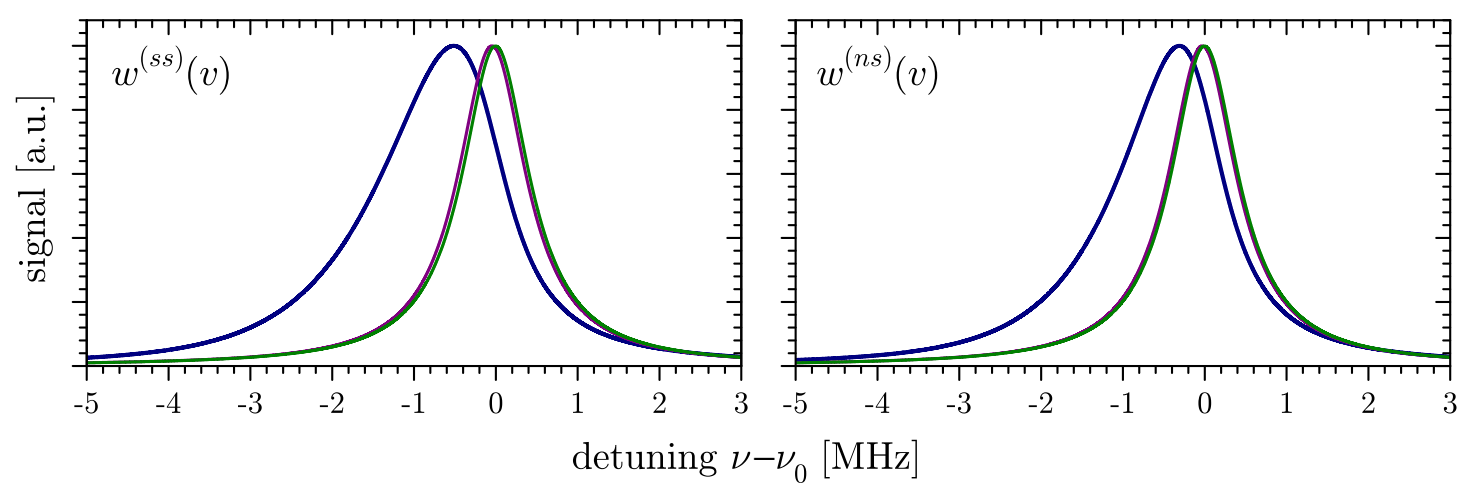

Figure 5.16.: Left: Examples of the line shape functions obtained with the weighting function $w^{(s s)}(v)$ (see (5.18) and (5.31)). Right: the same for the velocity distribution $w^{(n s)}(v)$ (see (5.19) and (5.31)). The lines are plotted with a natural line width of $\gamma=$ $1.0050 \mathrm{MHz}$ and temperatures of $T=2000 \mathrm{~K}$ (in blue, for visualization of the asymmetry) as well as for $T=300 \mathrm{~K}$ (purple) and $T=7 \mathrm{~K}$ (green). At $300 \mathrm{~K}$ one can see the secondorder Doppler shift even at the $\mathrm{MHz}$ scale. At $7 \mathrm{~K}$ the lines are indistinguishable from a Lorentzian.

to the experimental procedure, we define the line shift as obtained by fitting an undistorted Lorentzian line shape (4.1) to the distorted line shapes (5.31) and (5.32). This method is most sensitive to the inflection points and hence it agrees well with the mean shift of the half-width points.

This instrumental definition of the line shift does depend slightly on the details and range of the line sampling. Therefore, we use the experimental line sampling grid as described in section 4.1 also for the modelling. This grid may not always be perfectly aligned to the unperturbed transition frequency in the experiment, mostly because of the free running $f_{\text {rep }}$ (see section 3.1). For the current data set, the line sampling grid was detuned by $+54 \mathrm{kHz}$ on average with a standard deviation of $70 \mathrm{kHz}$ from the unperturbed transition frequency. To estimate the resulting line shape bias, we compute the line shapes (5.31) and (5.32) on the experimental line sampling grid with a randomly selected number of valid data points according to the experimental distribution shown in Fig. 4.1. In addition, we shift this grid by a random offset picked from a Gaussian distribution centered at $+54 \mathrm{kHz}$ with a standard deviation of $70 \mathrm{kHz}$. We repeat this 10,000 times for a given temperature and fit the line shapes with a Lorentzian to determine the line center, just as it is done in the experiment. We assume a peak count rate of $5 \mathrm{kHz}$ (see table 3.1) and use the corresponding shot noise for weighting. The result is shown in Fig. 5.17. The line shape bias becomes an issue at higher temperature because one has to assume a certain velocity distribution for its correction. With our Monte Carlo simulation, these line distortions are taken into account, including their cross-talks (see section 5.11). However, it is negligible for the data that we evaluate $(T \leq 30 \mathrm{~K})$. 


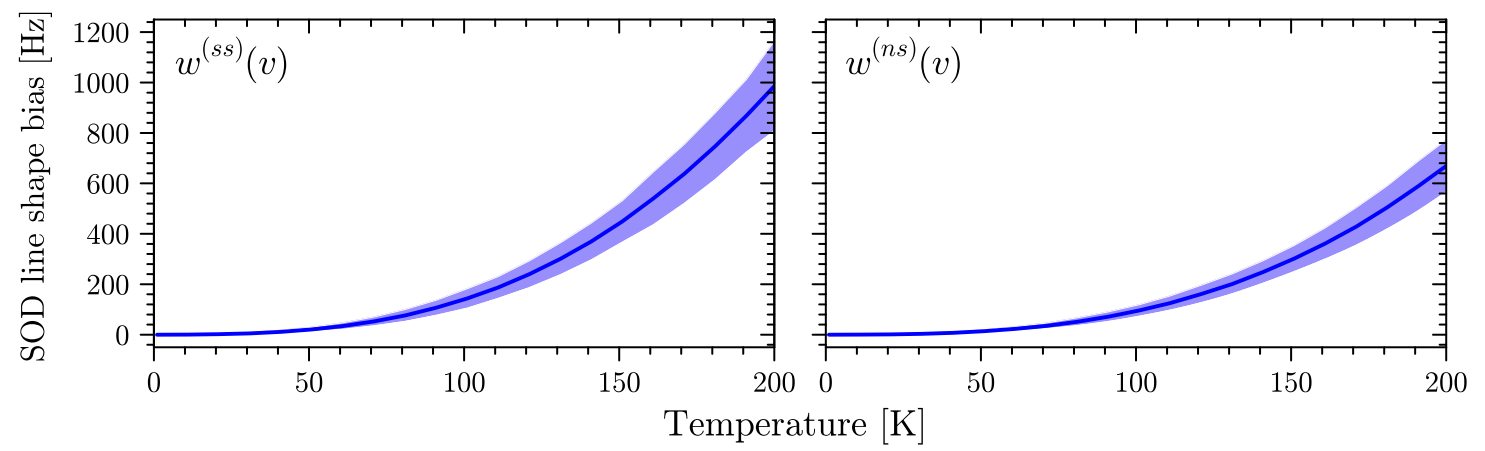

Figure 5.17.: Line shape contribution to the second-order Doppler (SOD) shift (at the atomic frequency) estimated by generating line shapes that follow from the weighting functions $w^{(s s)}(v)$ and $w^{(n s)}(v)$ (see (5.18) and (5.19)) and fitting a Lorentzian (4.1) to find the line center. The SOD line shift is plotted relative to velocity averaged SOD of $-(3 / 2)\left(v_{0}^{2} / c^{2}\right) \nu_{0}$ and $-\left(v_{0}^{2} / c^{2}\right) \nu_{0}$ respectively. The line sampling frequencies are chosen in accordance with the experiment as described in the text. The blue regions are the $1 \sigma$ standard deviations due to the assumed fluctuation of the line sampling grid (position and number).

\subsection{Quantum interference}

Another asymmetric line shape distortion is due to cross damping or quantum interference (QI) [70-73]. Quantum paths leading from the ground state $1 \mathrm{~S}(F=1)$ via several excited states $3 \mathrm{~S}$ and $3 \mathrm{D}$ to the final states $2 \mathrm{P}$ may interfere. As with any interference, this takes place only when the same initial and the same final states are connected via indistinguishable paths. Therefore only the components a) and d) in Fig. 3.2 contribute to QI. Another way to look at this effect is to consider the interference of the emitting dipoles. When driven with the same comb mode pair, these dipoles perform forced oscillations with identical frequencies, although not all with a larger resonant amplitude. The result is static spatial interference pattern that does however depend on the detuning, because the phase of the dipoles depend on it. The result is a line distortion that one observes in a certain direction, i.e. with a point-like detector. This distortion vanishes if one collects all the light in a full solid angle of $4 \pi$ sr. In the quantum path picture, paths with identical initial and identical final states (fine, hyperfine and Zeeman levels) give rise to a static interference pattern, because all of these dipoles emit with the same frequency which is given by the laser frequency minus the difference of the initial and the final state energy. Depending on whether the dipoles correspond to $\sigma_{ \pm}$or $\pi$ polarization, their spatial emission pattern is different which results in a phase and detuning dependent modification of the total spatial emission pattern. The order of magnitude of the associated line pulling obtained with a point-like detector is $\sqrt{a_{1}} \gamma \gamma_{1} / 2 \Delta$ where $\gamma_{1}$ and $a_{1}$ are the width and the relative line amplitude of the perturbing line (see (4.1)) that is separated by $\Delta[70]$. In the context of direct comb spectroscopy it is impor- 


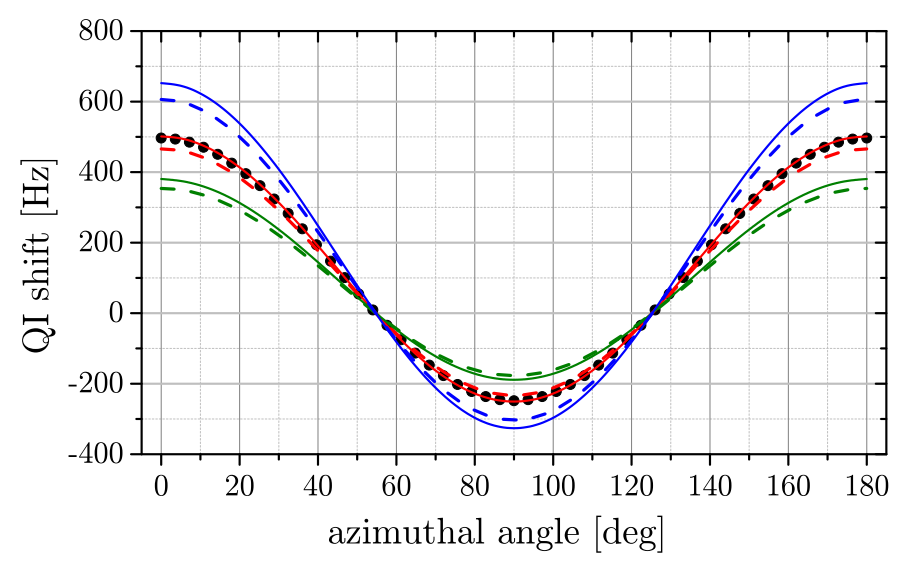

Figure 5.18.: Quantum interference (QI) shift (measured at the atomic frequency) obtained by fitting a Lorentzian to the line shapes that include quantum interference. The experimental line sampling grid (see section 4.1) has been used in this simulation. Using another line sampling, this shift can be significantly larger, depending how dense the frequency axis is sampled. The azimuthal angle is taken with respect to the atomic beam axis and lies close to $90^{\circ}$ for our geometry. The black circles show the results from perturbation theory as described in [47] but updated for $\sigma_{+} / \sigma_{-}$polarization and for a temperature of $5 \mathrm{~K}$. The red, green and blue curves are results from the Monte Carlo simulations that solve the optical Bloch equation for the three detectors $j=1, j=2$ and $j=3$ respectively (see Fig. 3.9). With this, non-stationary contributions, such as time-of-flight broadening and also saturation are included in the quantum interference. The solid curves are for a point-like detector (as in the perturbative description), while the dashed curves take the finite solid angle of the light collecting lenses into account (see section 3.6). The secondorder Doppler shift and AC-Stark shift have been turned off in these simulations to isolate the QI frequency shift. The uncertainty of the Monte Carlo simulation is about $10 \mathrm{~Hz}$, so that the lack of precise knowledge of the geometry, velocity distribution etc. sets the limit.

tant to note that $\Delta$ is the real line separation, not the comb folded line separation. In that sense the component closest to the main resonance is labeled d) in Fig. 3.2 for which we have $\Delta=2917 \mathrm{GHz}$ and $\gamma_{1}=10.3 \mathrm{MHz}$. To take the unequal line amplitudes into account, the order of magnitude estimation has to be multiplied by the square root of the line peaks resulting in $\approx 900 \mathrm{~Hz}$.

This coherent line distortion is asymmetric and therefore requires the same treatment as the SOD discussed above. Previously, we have analyzed this effect within the framework of perturbation theory for our experimental setting [47] - linear polarized laser beams and a room temperature atomic beam. With the counter-propagating $\sigma_{+}$and $\sigma_{-}$polarizations, the QI frequency shift is actually only half as large with the opposite sign as Fig. 5.18 shows. Note that the results in [47] are presented in terms of the laser frequency while here we plot it in terms of the atomic frequency. In the same figure we also show the QI shift obtained with our Monte Carlo simulations that also takes into account the time-of-flight and saturation. In addition, we can 
include the solid angle of the NA $=0.68$ lenses that reduces this effect further. Even without this reduction, the quantum interference frequency shift is small but we still correct our final result by adding $0.25 \mathrm{kHz}$.

Additional asymmetric line distortions arise from time-of-flight and power broadening. These are small compared to the natural line width as the typical line scan in Fig. 4.2d shows. In fact, the lines recorded at low temperatures, with low laser powers, and the down stream detector $(j=2)$ have line widths that are statistically indistinguishable from the natural line width. Moreover, these broadenings are symmetric and hence do not produce an additional line shape contribution to the shift discussed above.

\subsection{Incoherent line pulling}

We define incoherent line pulling as effects where the center of the line no longer coincides with the transition energy because of a sloping baseline caused by the presence of other lines. In contrast to the previous section, we now discuss the situations where there is no static interference between these lines, so that the sloping baseline is the only pulling mechanism [70]. One could eliminate this effect by accounting for the other components with an appropriate fitting function. For simplicity, we are fitting a single Lorentzian only (see section 4.2) and, as in the previous section, quantify the line pulling by employing an instrumental definition obtained by the fitting result. The incoherent line pulling is, in general, significantly smaller than the coherent line pulling through quantum interference [70].

\subsubsection{Other line components}

As seen in Fig. 3.2, other fine and hyperfine components are folded into the observed spectrum because of the comb excitation. The ps-comb has a spectral envelope that is about $150 \mathrm{GHz}$ wide and therefore covers all components of the $n=1 \rightarrow n=3$ transition (see Fig. 3.3). We have fine tuned the repetition rate in order to isolate the main component of interest (see section 3.2). Nevertheless, we have to investigate the remaining line pulling. The different components that appear in the wrapped spectrum are excited with different comb pairs. This means their spatial interference pattern is not static but beats at multiples of the repetition rate and the quantum interference effect averages out during the 1 sec gate time of our detectors. The resulting line pulling can be easily determined by modeling a spectrum as is shown in Fig. 3.2 consisting of 6 Lorentzians (with appropriate amplitude and widths) and then fitting a single Lorentzian only (within the spectral range and with the line sampling used in the experiment). In this way, we obtain a negligible line pulling of $-48.5 \mathrm{~Hz}$, which justifies the use of a single Lorentzian for a simplified data analysis.

Another source of perturbing peaks in the spectrum are the imperfectly suppressed modes of the laser. As detailed in section 3.2, the doubling cavities are operated 
at twice the lasers repetition rate and hence also double the mode spacing from 78.8 MHz to $157.6 \mathrm{MHz}$. As shown in section 3.3, the suppression of the $78.8 \mathrm{MHz}$ spaced modes relative to $157.6 \mathrm{MHz}$ modes is given by $T^{4}$, where $T=30 \%$ is the power transmission of the input couplers of both of the doubling cavities. The $205 \mathrm{~nm}$ enhancement cavity is also operated at twice the laser repetition rate, even though we effectively have four times the repetition rate for the Doppler-free excitation by circulating two pulses simultaneously in this cavity. Assuming the $157.6 \mathrm{MHz}$ spaced resonant modes are impedance-matched, they are power enhanced by $1 / T_{1}$ whereas the off-resonant $78.8 \mathrm{MHz}$ spaced modes are further suppressed with the inverse factor. Here, $T_{1}=6.2 \%$ is the input coupler transmission of the $205 \mathrm{~nm}$ enhancement cavity. In total the unwanted modes are suppressed by $m=T^{4} T_{1}^{2}=3.1 \times 10^{-5}$, so that we actually do not see them in the experimental data. Nevertheless we need to estimate their possible line pulling effect. We do this by solving the optical Bloch equations of the full level scheme (see section 2.5) by assuming a superimposed comb with a $78.8 \mathrm{MHz}$ mode spacing that is suppressed by the above factor $\mathrm{m}$. Analyzing this data exactly as the experimental data, we obtain a line pulling of roughly $\delta \nu=4 \mathrm{kHz} \times m=0.12 \mathrm{~Hz}$, which can be safely ignored.

If the exciting frequency comb would have residual modes with a quarter integer mode number, i.e. with a $78.8 \mathrm{MHz}$ spacing, we can determine where the folded line components a), b), c), d) and e) from Fig. 3.2 would reappear in the spectrum. With the quarter integer mode numbers of $+12.75,+13.75,+17.25,+9.25$ and +4.25 they are expected at a detuning from the main resonance of $-18.7 \mathrm{MHz}$, $+4.7 \mathrm{MHz},-18.3 \mathrm{MHz},+1.1 \mathrm{MHz}$ and $+28.2 \mathrm{MHz}$ respectively. Unlike the extra line components discussed below, some of these detunings are too small to limit the line pulling just with the large line separation. One might find a limit by investigating the residuals in Fig. 4.5, but the amplitude limit presented above provides a tighter limit in this case.

As explained in section 3.2, the propagation direction of the colliding pulses and their polarizations alternate. In the ideal case a $\sigma_{+}$polarized pulse from the left and a $\sigma_{-}$polarized pulse from the right alternates with a $\sigma_{-}$polarized pulse from the left and a $\sigma_{+}$polarized pulse from the right. The electric field vectors add, forming a helix of a linear polarized standing field within the PCV whose position alternates between collisions. In contrast, the Doppler free two-photon Rabi frequency (5.2) is obtained as the product of the counter-propagating fields. Therefore the Rabi frequency is not necessarily alternating, even if the electric field vector is. To see that this is indeed the case, independent of polarization, we write the counter-propagating pulses as $\vec{E}_{+}=\vec{e}_{+} A(t-z / c) \cos \left(\omega_{c} t-k z-\varphi / 2\right)$ and $\vec{E}_{-}=\vec{e}_{-} A(t+z / c) \cos \left(\omega_{c} t+\right.$ $k z+\varphi / 2)$ with $z=0$ being center of the enhancement cavity, $t=0$ the time of the first pulse collision, $\vec{e}_{ \pm}$the polarizations, $A(t)$ the pulse envelope and $\varphi$ a possible phase shift of the carrier frequency $\omega_{c}$. After one round trip time $T$, the propagation direction is exchanged $(z \rightarrow-z)$ and the pulses are delayed: $\vec{E}_{+}=\vec{e}_{-} A(t+T+$ $z / c) \cos \left(\omega_{c}(t+T)+k(z-c T)-\varphi / 2\right)=\vec{e}_{-} A(t+z / c) \cos \left(\omega_{c} t+k z-\varphi / 2\right)$ and $\vec{E}_{-}=$ 
$\vec{e}_{+} A(t+T-z / c) \cos \left(\omega_{c}(t+T)-k(z+c T)+\varphi / 2\right)=\vec{e}_{+} A(t+z / c) \cos \left(\omega_{c} t-k z+\varphi / 2\right)$, where we assumed that the pulse envelopes are the same and periodic with $T$. Within the rotating wave approximation (for two-photon transitions) we keep only terms in the product of the fields that oscillate with $2 \omega_{c}$. This means that $k z$-term drops out as it should (Doppler-free) and so does the $\varphi$-term. More important to the discussion here is that the Rabi frequency is the same for the first pulse and after one round trip. Hence, within the rotating wave approximation, this mechanism does not lead to the emergence of a $157.6 \mathrm{MHz}$ comb spacing in the recorded spectrum.

The discussion of the last paragraph assumes that the timing of the pulses is such that they always meet at $z=0$. However, the delay stage that is used to generate the counter-propagating pulses may not be perfectly adjusted. As a result one of the pulses is advanced and the other delayed by say $\delta t$ which leads to a factor $A(t-\delta t / 2-z / c) A(t+\delta t / 2+z / c)$ in the above Rabi frequency for the first pulse collision. After one round trip the propagation direction is flipped and the pulse is delayed such that the product of the envelopes becomes $A(t-\delta t / 2+(z-c T) / c) A(t+$ $\delta t / 2-(z+c T) / c)=A(t-\delta t / 2+z / c) A(t+\delta t / 2-z / c)$, where we assumed again that each pulse train is periodic. This means that the position of the PCV alternates between $z= \pm c \delta t / 2$, i.e. by $\Delta z=c \delta t$ between subsequent pulse collision events. As a consequence, atoms at a fixed position other than $z=0$ experience an amplitude modulated Rabi frequency at 157.6 MHz. We measure and set an upper limit on the timing error of $|c \delta t|<40 \mu \mathrm{m}$ as described in section 3.2. Then we model this effect by solving the optical Bloch equations of the full level scheme and analyse the generated data exactly like the real experimental data. In this way, we find a frequency pulling of $\delta \nu=-0.0019\left(\mathrm{~Hz} / \mu \mathrm{m}^{2}\right) \times(c \delta t)^{2}=-3.0 \mathrm{~Hz}$, which can be safely ignored.

In addition we can also limit the line pulling $157.6 \mathrm{MHz}$ comb component that emerges through any mechanism with the experimental data. The folded line components a), b), c), d) and e) from Fig. 3.2 reappear with half integer mode number off-sets of $+12.5,+13.5,+17.5,+9.5$ and +4.5 at a detuning from the main resonance of $+60.1 \mathrm{MHz},+83.5 \mathrm{MHz},-97.1 \mathrm{MHz},-77.1 \mathrm{MHz}$ and $+50.6 \mathrm{MHz}$ respectively. Hence, these additional line components, if they exist, are separated from the main resonance by a detuning comparable to the other fine and hyperfine components discussed above. With their amplitude significantly lower, the resulting line pulling is also significantly lower and therefore negligible.

\subsubsection{Forbidden $\Delta F=1$ components}

Another possible source of incoherent line pulling may be due to the forbidden $\Delta F=1$ hyperfine components (see level scheme in Fig. 3.2). Since no orbital angular momentum is involved in the 1S-3S transition, the electron spin must flip relative to the nuclear spin for a $\Delta F \neq 0$ component. This is not possible with the electric dipole operator that drives the two-photon transition [42]. Therefore these components are forbidden for any laser polarization such that we do not have to 
discuss polarization imperfections in this context. However, higher order transition amplitudes or perturbations by stray fields allow these components to some extent.

The location of these extra components within the spectrum are determined by the very well known $1 \mathrm{~S}$ and $3 \mathrm{~S}$ hyperfine splittings and the mode spacing of our frequency comb. The $1 \mathrm{~S}(F=1) \rightarrow 3 \mathrm{~S}(F=0)$ component appears at a detuning of $-52.609473 \mathrm{MHz}[10]$, which is the excited state hyperfine splitting $A_{3 \mathrm{~S}}$, while the $1 \mathrm{~S}(F=0) \rightarrow 3 \mathrm{~S}(F=0)$ component appears at a detuning of $+2.005752 \mathrm{MHz}$, which is the ground state hyperfine splitting [78] $A_{1 \mathrm{~S}}$ minus 4.5 times the mode spacing of the comb. Both forbidden components are closer to the main resonance than the allowed components (see Fig. 3.2). To estimate the resulting line pulling we ignore the allowed components and again generate artificial data that contains one of the forbidden components with a line amplitude ratio $r$ and fit a single Lorentzian. In doing so (using the experimental line sampling), we find $\delta \nu=40 \mathrm{kHz} \times r$ for the $1 \mathrm{~S}(F=0) \rightarrow 3 \mathrm{~S}(F=1)$ component and $\delta \nu=-1.8 \mathrm{~Hz} \times r$ for the $1 \mathrm{~S}(F=1) \rightarrow 3 \mathrm{~S}(F=$ $0)$, linear in the relative amplitude $r$ with very good approximation. The latter line pulling can be neglected given the observation that $r \ll 1$, while the former requires a more careful estimation of $r$. There are several conceivable mechanisms that may allow this forbidden transition:

1. Only two-photon transitions are resonant and can give rise to a line in close proximity with the main line. This might be combinations of the electric E1 and the magnetic M1 dipole or higher multipole operators. Relativistic corrections to the matrix elements and levels might also be considered to allow the forbidden transition. However, it follows from the Furry theorem that all two-photon excitations of the forbidden line without external perturbations are excluded [60]. One way to read this theorem is that an odd number of photons cannot be converted to an even one without changing a state of something else (the atom). The two-photon excitation $1 \mathrm{~S}(F=0) \rightarrow 3 \mathrm{~S}(F=1)$ of any type and subsequent single-photon decay via $3 \mathrm{~S}(F=1) \rightarrow 1 \mathrm{~S}(F=0)$, that is certainly allowed, would represent such a process. Hence, we can restrict the discussion here to the role of external fields.

2. A stray electric field $E$ mixes the $3 \mathrm{P}$ levels to the $3 \mathrm{~S}$ levels and allows the $1 \mathrm{~S}(F=0) \rightarrow 3 \mathrm{~S}(F=1)$ transition. As derived in section 5.5, this admixture leads to a Rabi frequency of $\Omega_{1 \mathrm{~S}-3 \mathrm{P}}=X_{3} E \Omega_{1 \mathrm{~S}-3 \mathrm{~S}}=3.4 \times 10^{-4} \Omega_{1 \mathrm{~S}-3 \mathrm{~S}}$. Again, the line amplitude ratio is given by the square of the ratio of the Rabi frequencies. The resulting line pulling of $\delta \nu=40 \mathrm{kHz} \times\left(3.4 \times 10^{-4}\right)^{2}$ is negligible.

3. Unlike a stray electric field, a stray magnetic field $B$ mixes the 3 S state with the same parity states so that the $3 \mathrm{~S}(F=1)$ state acquires a $3 \mathrm{~S}(F=0)$ contribution. The Breit-Rabi formula (5.26) gives the energy of the perturbed state. With the same formalism the amplitude of the admixture can be determined (see for 
example [77]):

$$
|\psi\rangle=|3 \mathrm{~S}(F=1, M=0)\rangle+\frac{\sqrt{1+x(A)^{2}}-1}{x(A)}|3 \mathrm{~S}(F=0, M=0)\rangle
$$

with $x(A) \propto B$ as defined in section 5.6 and with the measured stray field given there we get $x(A)=7.4 \times 10^{-4}$. This admixture allows for a resonant $1 \mathrm{~S}(F=0) \rightarrow 3 \mathrm{~S}(F=1)$ transition. Therefore the square of the coefficient $\left(\sqrt{1+x(A)^{2}}-1\right) / x(A) \approx x(A) / 2 \approx \mu_{B} B / A$ directly gives the line amplitude ratio $r$ because the Rabi frequencies of the S-S transitions do not depend on polarization or on the hyperfine component [30]. With $A=A_{3 \mathrm{~S}}$ the resulting line pulling of $\delta \nu=40 \mathrm{kHz} \times\left(3.7 \times 10^{-4}\right)^{2}$ is negligible.

4. Leakage from the $2.4 \mathrm{GHz}$ radio frequency discharge that is used to dissociate $\mathrm{H}_{2}$ molecules into atoms, may cause a perturbation that can allow the forbidden transition. This frequency is much smaller than the optical frequency and therefore mostly couples magnetically to the hyperfine splitting. The treatment is analogous to the previous item, but with the mixing coefficient replaced by $\mu_{B} B_{\mathrm{RF}} /(A \pm 2.4 \mathrm{GHz})$ summing over the two signs (no rotating wave approximation). The vacuum chamber attenuates the radio frequency magnetic field down to $B_{\mathrm{RF}}=E_{\mathrm{RF}} / c=10 \mu$ Gauss (see section 5.2 and [64]). With $A=A_{3 \mathrm{~S}}$ the resulting line pulling of $\delta \nu=40 \mathrm{kHz} \times\left(2.6 \times 10^{-6}\right)^{2}$ is negligible.

5. The exciting laser represents another perturbation that may allow the $1 \mathrm{~S}(F=$ $0) \rightarrow 3 \mathrm{~S}(F=1)$ that is forbidden in second order perturbation theory. In third order there is no path connecting an $\mathrm{S} \rightarrow \mathrm{S}$ transition that is compatible with the parity selection rules. Hence the lowest order is the forth order perturbation via the close to resonant $3 \mathrm{~S}(F=0)$ excitation paths $1 \mathrm{~S}(F=0) \rightarrow n \mathrm{P} \rightarrow$ $3 \mathrm{~S}(F=0) \rightarrow n^{\prime} \mathrm{P} \rightarrow 3 \mathrm{~S}(F=1)$ with off resonant intermediate states $n \mathrm{P}$, and $n^{\prime} \mathrm{P}$, where $n$ and $n^{\prime}$ are summed over all bound and continuum states. Similar to the fourth order treatment of the AC-Stark shift in section 5.2 [63] we obtain for the Rabi frequency

$$
\Omega_{4} \approx\left(\omega_{r} \tau\right)^{2}\left(\frac{e E_{0}}{6 \hbar}\right)^{4} S_{1}\left(S_{2}+S_{3}\right) \sum_{n_{1}, n_{2}} \frac{e^{-\left(n_{1}^{2}+n_{2}^{2}\right)\left(\omega_{r} \tau / 2\right)^{2}}}{A_{1 \mathrm{~S}}-\left(n_{1}-n_{2}\right) \omega_{r}}
$$

with

$$
S_{1} \equiv \sum_{n} \frac{\mu_{3 \mathrm{~S}, n \mathrm{P}} \mu_{n \mathrm{P}, 1 \mathrm{~S}}}{\omega_{n \mathrm{P}, 1 \mathrm{~S}}-\omega_{c}} \quad S_{2} \equiv \sum_{n} \frac{\mu_{3 \mathrm{~S}, n \mathrm{P}}^{2}}{\omega_{n \mathrm{P}, 1 \mathrm{~S}}-\omega_{c}} \quad S_{3} \equiv \sum_{n} \frac{\mu_{3 \mathrm{~S}, n \mathrm{P}}^{2}}{\omega_{n \mathrm{P}, 1 \mathrm{~S}}-3 \omega_{c}}
$$

where $\mu$ are the corresponding dipole matrix elements, $\omega_{c}$ is the comb carrier frequency, $\omega_{r}$ is the repetition rate, and the other labeled $\omega$ are the corresponding transition frequencies. By evaluating (5.34) with a peak field of 
$E_{0}=2.6 \mathrm{MV} / \mathrm{m}^{2}$, which corresponds to the mean experimental conditions, we find $\Omega_{4} \approx 2 \pi \times 6.5 \times 10^{-4} \mathrm{~Hz}$. Under the same conditions the Rabi frequency of the main $1 \mathrm{~S}(F=1) \rightarrow 3 \mathrm{~S}(F=1)$ component is $\approx 2 \pi \times 60 \mathrm{~Hz}$. The resulting line pulling of $\delta \nu=40 \mathrm{kHz} \times\left(6.5 \times 10^{-4} / 60\right)^{2}$ is negligible.

Besides estimating possible mechanisms that would allow the forbidden hyperfine components, we can also set a limit from our data. Accumulating the data recorded with a nozzle temperature of $7 \mathrm{~K}$ and a nozzle distance of $d=27.1 \mathrm{~mm}$ allows to generate the averaged line scan presented in Fig. 4.5. Thanks to the very good signal to noise ratio of this averaged line, we can put an experimental limit on a possible weak additional line at a laser detuning of $2 \mathrm{MHz}$. From the figure we can read off a maximum line amplitude ratio of $r=0.002$ which would correspond to frequency pulling of $80 \mathrm{~Hz}$. With the arguments above we believe that this pulling is actually even smaller and hence neglect it.

\subsubsection{Cavity modulation side bands}

Another possible source of sideband induced line distortions is due to the modulation applied for stabilizing the $205 \mathrm{~nm}$ enhancement cavity (see section 3.4). This cavity is kept on resonance using the dither lock technique. For this purpose, the input coupling mirror is mounted on a ring-shaped piezo transducer, whose voltage is modulated at various frequencies between $\omega_{m}=2 \pi \times 60 \mathrm{kHz}$ and $90 \mathrm{kHz}$. By demodulating a portion of the detected cavity transmission and mixing it with a TTL signal at the modulation frequency, we generate an error signal for the feedback loop. Acoustic resonances of the mirror mounts make it difficult to estimate the amplitude of the cavity length modulation $\Delta d$ from the applied voltage and the manufacturer supplied piezo properties. However, we can place an upper limit on this amplitude by observing that the relative fluctuation of the transmitted laser power in the locked condition is not more than $2 \%$. This noise, which is dominated by mechanical vibration, is significantly larger than the component at $\omega_{m}$. By expanding (3.8) about the resonance, we find the corresponding modulation amplitude with the cavity properties given in section 3.4 :

$$
\Delta d<\sqrt{2 \%} \frac{\lambda}{2 \pi} \frac{1-\sqrt{R_{1} R_{2}}}{2\left(R_{1} R_{2}\right)^{1 / 4}}=0.16 \mathrm{~nm} .
$$

Modulating the cavity length does not only modulate the amplitude of the intra cavity field (as seen be the locking electronics) but also the phase of that field. Both, pure amplitude and pure phase modulation give rise to a symmetric sideband spectrum and hence do not produce a line pulling. Only the interplay between both types of modulation could make the sideband spectrum asymmetric. In the following we show that for a perfect cavity not even this interplay gives rise to an asymmetric sideband spectrum. 
To take both types of modulation into account we are rewriting (3.8) for the intra cavity steady state field that propagates in one direction

$$
E \propto \frac{e^{-i \omega_{0} t}}{1-\sqrt{R_{1} R_{2}} e^{i \theta}}
$$

with the carrier frequency of the impinging laser $\omega_{0}$ and the round-trip phase $\theta=$ $2 \pi d / \lambda$. The Rabi frequency that drives the Doppler-free 1S-3S transition is proportional to the product the two counter-propagating fields, which are both assumed to be of the form (5.37) - a good approximation for this purpose. Modulating the cavity length with a modulation index $M=2 \pi \Delta d / \lambda$ is described as

$$
\theta=\theta_{0}+M \sin \left(\omega_{m} t\right)
$$

The round trip phase $\theta_{0}$ at the locking point is ideally at an integer of $2 \pi$. Note that the modulation index here is not the phase modulation index of the intra-cavity light, but the modulation index of the cavity length. Since the modulation frequency is much lower than the inverse photon life time of the cavity (50 ns), we can use the steady state field. Using

$$
e^{i M \sin \left(\omega_{m} t\right)}=\sum_{n=-\infty}^{+\infty} J_{n}(M) e^{i n \omega_{m} t} \quad \text { and } \quad \frac{1}{1-x}=\sum_{k=0}^{\infty} x^{k}
$$

with the Bessel functions $J_{n}(M)$ we find:

$$
E \propto e^{-i \omega_{0} t} \sum_{k=0}^{\infty}\left(\sqrt{R_{1} R_{2}} e^{i \theta_{0}} \sum_{n=-\infty}^{+\infty} J_{n}(M) e^{i n \omega_{m} t}\right)^{k}
$$

From this expression we can see that, in principle, all harmonics of $\omega_{m}$ are present, even though they fall off quickly if the modulation index is small. It is also seen that each sideband frequency at multiplies of $\omega_{m}$ is a result of many combinations of terms. To investigate the symmetry of the sideband spectrum we use the symmetry of the Bessel function $J_{-n}(M)=(-1)^{n} J_{n}(M)$. This means that positive and negative sidebands have the same amplitude and differ at most through an inverted sign. Hence the power spectrum is always perfectly symmetric without any line pulling.

To model possible deviations from this perfectly symmetric case, we take advantage of the smallness of $M=2 \pi \Delta d / \lambda<0.0049$ estimated above and sum only the first-order sidebands of (5.40) - for example by expanding (5.37):

$$
E \approx \frac{e^{-i \omega_{0} t}}{1-\sqrt{R_{1} R_{2}} e^{i \theta_{0}} J_{0}(M)}+\sqrt{R_{1} R_{2}} e^{i \theta_{0}} J_{1}(M) \frac{e^{-i\left(\omega_{0}-\omega_{m}\right) t}-e^{-i\left(\omega_{0}+\omega_{m}\right) t}}{\left(1-\sqrt{R_{1} R_{2}} e^{i \theta_{0}} J_{0}(M)\right)^{2}}
$$

Assuming that $\theta_{0}$ is close to its locking point, we calculate a power ratio of $r=0.0012$ of the sidebands relative to the carrier. Again, in the symmetric case we expect a 
small line broadening but no frequency shift. To obtain an upper limit on the possible line shift, we now assume the maximally asymmetric case of only one sideband. Fitting a single Lorentzian to a superposition of two Lorentzians separated by $\omega_{m}$ with a relative amplitude of $r$ results in a line pulling of $\delta \nu \approx 4 r \omega_{m} / 2 \pi$, which amounts to $0.44 \mathrm{kHz}$ for the largest modulation frequency used. Since the maximum asymmetry is suredly an overestimate, we believe that the true shift is much smaller and therefore neglect this lineshift.

\subsection{Tilted wave fronts}

Doppler-free excitation requires that the two exciting wave vectors are pointing in opposite directions $\left(\vec{k}_{+}=-\vec{k}_{-}\right)$. For this, the wavefronts of the forward and backward propagating waves must be matched. If this condition is violated we expect another residual first-order Doppler shift (different from CIFODS) for an atom with a velocity $\vec{v}$ of:

$$
2 \pi \delta \nu_{D S}=\Delta \vec{k} \cdot \vec{v}=2 k v \sin (\varepsilon) \sin (\alpha)
$$

where $\Delta \vec{k}=\vec{k}_{+}+\vec{k}_{-}, \varepsilon$ is the deviation of the angle between the local waves vectors from $180^{\circ}, 90^{\circ}-\alpha$ is the angle of the atomic trajectory with respect to $\Delta \vec{k}$, and $k=\left|\vec{k}_{-}\right|=\left|\vec{k}_{+}\right|$. An example for this situation is sketched in Fig. 5.19.

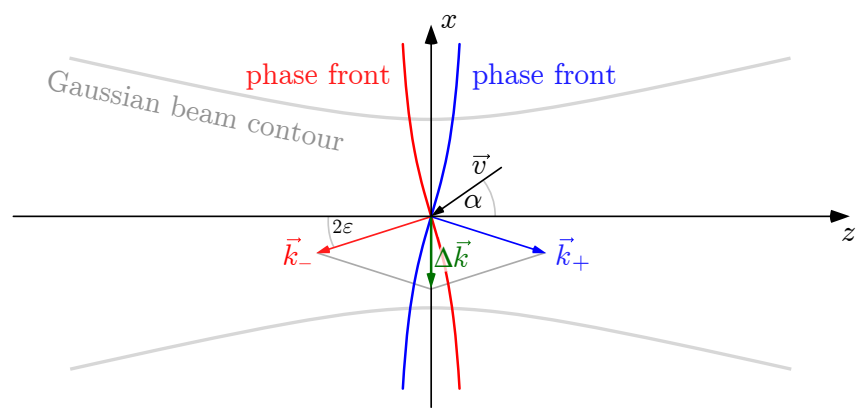

Figure 5.19.: Residual first-order Doppler shift due to misaligned wave fronts that assume a $\tan ( \pm k z)$ shape for the considered superposition of the $\mathrm{TEM}_{00}$ and the $\mathrm{TEM}_{01}$ GaussHermite modes. The counter-propagating waves have local wave vectors $\vec{k}_{+}$and $\vec{k}_{-}$. The deviation $\alpha$ of the angle of the atomic velocity vector $\vec{v}$ with the local $\Delta \vec{k}=\vec{k}_{+}+\vec{k}_{-}$from $90^{\circ}$ determines the local Doppler shift. While all TEM modes share the same optical axis (as sketched), the considered superposition actually tilts this axis.

The $205 \mathrm{~nm}$ enhancement cavity helps to generate counter-propagating waves. However, due to the finite finesse the wavefronts are not necessarily perfectly matched. An aligned cavity enhances the mode matched fraction of the input wave and, at the same time, suppresses the part that is not matched. Therefore, even a low-finesse cavity can significantly suppress the first-order Doppler effect. It might seem obvious that there are two mechanisms that can lead to a phase front mismatch between 
the forward and backward propagating waves - a misaligned cavity and a less than perfect spatial mode matching. However, for sufficiently large mirrors, the cavity can not technically be misaligned. The line connecting the centers of curvatures of the two mirrors always forms a perfectly aligned cavity axis [79]. Hence in the following we assume a perfectly aligned cavity and describe all misalignments as a mismatch to the $\mathrm{TEM}_{00}$ mode, i.e. an admixture of higher-order transverse modes. With this, we can quantify this mismatch by observing the transverse mode spectrum when scanning the cavity.

All transverse modes possess the same spatial wavefronts, except for the Gouy phase which is not a radial phase. Therefore adding transverse modes to the main $\mathrm{TEM}_{00}$ mode with real coefficients cannot describe a tilt of the wavefront, only a displacement for odd modes, or an enlargement of the waist for even modes. However, adding an odd-order transverse mode with a purely imaginary coefficient tilts the resulting phase front because it introduces an asymmetric radial phase. Therefore, to place a limit on the residual first-order Doppler shift, we assume the coefficient to be purely imaginary and consider a superposition of the $\mathrm{TEM}_{00}$ and the $\mathrm{TEM}_{01}$ Gauss-Hermite modes given by

$$
E_{ \pm} \propto\left(\sqrt{1-\eta^{2}}+i 2 \eta \frac{x}{w_{0}}\right) e^{-\rho^{2} / w_{0}^{2} \pm i k z}
$$

where $\rho^{2}=x^{2}+y^{2}$ and $\eta$ is a real coefficient with $\eta=0$ corresponds to a pure $\mathrm{TEM}_{00}$ mode. The off-resonant coupling of the $\mathrm{TEM}_{01}$ mode (second term) and its relative Gouy phase certainly varies the phase of the superposition, but we assume a purely imaginary coefficient here as an upper limit for the residual first-order Doppler shift. One might argue that the odd transverse modes can easily be suppressed by tilting the input beam. However, this is only true for a clean $\mathrm{TEM}_{00}$ impinging mode. Walk-off in the second doubling stage adds significant beam distortions so that this is not the case here (see section 3.4).

Given the length of the pulse collision volume and the $\sim 200$ times larger Rayleigh length, we can assume a constant beam radius $w_{0}$. The shape $x(z)$ of the phase fronts of the field (5.43) is obtained with:

$$
\arg \left[E_{ \pm}\right]=\arctan \left(\frac{\sqrt{1-\eta^{2}} \sin ( \pm k z)+2 \eta\left(x / w_{0}\right) \cos ( \pm k z)}{\sqrt{1-\eta^{2}} \cos ( \pm k z)-2 \eta\left(x / w_{0}\right) \sin ( \pm k z)}\right)
$$

In the limiting cases, $\eta=0$ and $\eta=1$ (a pure $\mathrm{TEM}_{00}$ or $\mathrm{TEM}_{01}$ mode) this leads to the usual plane wave propagation phase $\pm k z$ with no first-order Doppler shift. For $0<\eta<1$, we can determine the shape of the phase front by resolving the zeros of $(5.44)$ :

$$
x(z)=-2 w_{0} \sqrt{1 / \eta^{2}-1} \tan ( \pm k z) \quad \text { for } 0<\eta<1
$$

In this case, the forward and backward waves travel with different phase fronts as sketched in Fig. 5.19, and hence give rise to a residual first-order Doppler shift. 
More generally we can use (5.10) to compute the residual first-order Doppler shift from the spatial phase variation of the Rabi frequency

$$
2 \pi \delta \nu_{D S}=\vec{v} \cdot \vec{\nabla}\left(\arg \left[E_{-} E_{+}\right]\right)=v_{x} \frac{4 w_{0} \eta \sqrt{1-\eta^{2}}}{w_{0}^{2}\left(1-\eta^{2}\right)+4 x^{2} \eta^{2}} \approx v_{x} \frac{4 \eta}{w_{0}},
$$

where $v_{x}$ is the transverse atomic velocity and the last expression assumes that $\eta \ll 1$ while $x$ is not very much larger than $w_{0}$. With this we can give an estimate of the residual first-order Doppler shift. The distance of the excitation region (PCV) and the nozzle is at least $d=19.1 \mathrm{~mm}$, the nozzle orifice diameter is $1.3 \mathrm{~mm}$. Since the laser beam goes through the nozzle, it cannot be displaced by more than the orifice radius minus the laser beam radius before it gets clipped. This means $|\alpha|<32 \mathrm{mrad}$ and hence $\left|v_{x}\right|<11 \mathrm{~m} / \mathrm{s}$ at the most probable thermal velocity of $340 \mathrm{~m} / \mathrm{s}$ at $7 \mathrm{~K}$. The parameter $\eta$ can be estimated from the observed relative power of the transverse modes and the expected suppression when locking the cavity to the $\mathrm{TEM}_{00}$ mode. With the cavity parameters presented in section 3.4, the power enhancement (3.8) on resonance is around 15 while the suppression of the off resonant $\mathrm{TEM}_{01}$ is 0.068 (with $\theta \approx \pi / 6$, i.e. about 6 transverse modes per free spectral range). At the same time, the transverse modes were observed to be suppressed by a factor of at least 10 when scanning over all resonances. Therefore we estimate $\eta \approx \sqrt{0.1 \times 0.068 / 15}=0.021$ through the square root of the observed mode overlap. The maximum residual first-order Doppler shift is estimated with (5.46) as $|\delta \nu| \approx 1.8 \mathrm{kHz}$. Since the transverse velocity $v_{x}$ occurs with both signs, only the asymmetric part of the atomic beam produces a real frequency shift. In addition, it changes sign during frequent realignments, so that we assume it averages to be insignificant for the final error analysis.

Besides the mode mismatch, imperfect alignment of the delay line that generates the counter -propagating pulses (see section 3.2 and Fig. 3.1) may give rise to its own residual first-order Doppler effect. If the two beams are not perfectly mode matched with respect to each other, they cannot, of course, be simultaneously and perfectly mode matched to the enhancement cavity. Therefore they are both described by an independent spectrum of higher order modes. However, the residual first-order Doppler shift reverses sign every other pulse because the $\sigma_{ \pm}$pulses come from alternating directions. The atoms are exposed to about 50 pulses per lifetime of the excited state. Hence, the maximum first-order Doppler shift due to the misalignment of the delay line is at about 50 times smaller than the value estimated above for a single mismatched beam.

We can also exploit the velocity dependence of any conceivable first-order Doppler effect to set another limit from the experimental data. To this end, we correct the measured frequencies of all scans for all systematics that are extrapolated in (4.4) and investigate a possible linear dependence on the most probable thermal velocity $v_{0}=\sqrt{2 k_{B} T / m}$ of the atoms of mass $m$. Figure 5.20 shows the result. The Doppler slope $+0.3(1.2) \mathrm{Hz} /(\mathrm{m} / \mathrm{s})$ is consistent with zero. Evaluating the $4.5 \mathrm{~K}, 7.0 \mathrm{~K}$, 


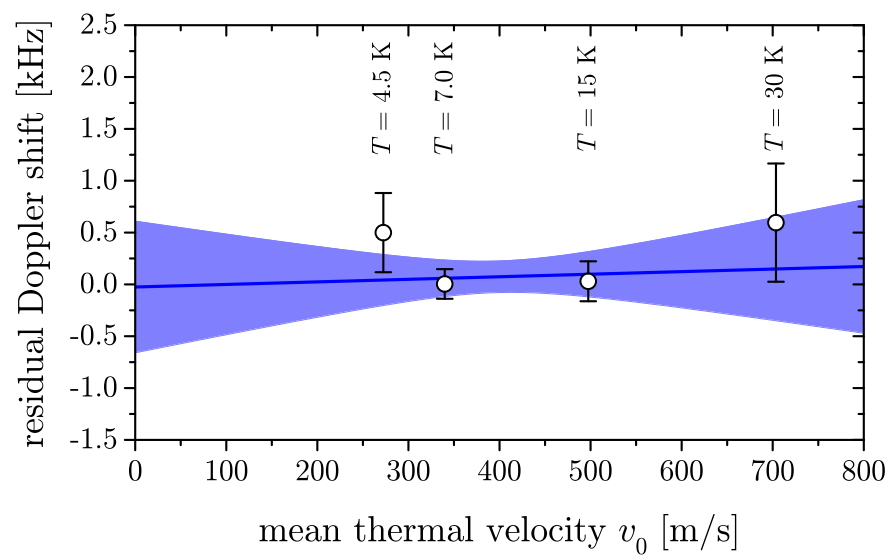

Figure 5.20.: Residual first-order Doppler shift measured at the atomic frequency relative to the final result of this work determined by correcting each line scan for all other systematic shifts including the extrapolated systematic shifts due to the CIFODS $\left(\kappa_{D S}\right)$, the second-order Doppler $\left(\kappa_{S O D}\right)$, the AC-Stark $\left(\kappa_{A C}\right)$ and the pressure shift $\left(\kappa_{P S}\right)$, using the results of the global fit in table 4.1. As for the final analysis, only the $4.5 \mathrm{~K}, 7.0 \mathrm{~K}$, $15 \mathrm{~K}$ and $30 \mathrm{~K}$ data are used to identify a possible linear dependence on the most probable velocity $v_{0}=\sqrt{2 k_{B} T / m}$ of the atoms. Fitting a linear function (blue line with $1 \sigma$ confidence interval) results in Doppler slope of $+0.3(1.2) \mathrm{Hz} /(\mathrm{m} / \mathrm{s})$ and an intercept of $-0.03(48) \mathrm{kHz}$. The error bars are obtained from a weighted average of lines scans that are assumed to be subject to shot noise only.

$15 \mathrm{~K}$ and $30 \mathrm{~K}$ data only, we obtain a weighted-average mean thermal velocity of $\left\langle v_{0}\right\rangle=397(56) \mathrm{m} / \mathrm{s}$, which results in a mean residual first-order Doppler shift of $0.12(0.46) \mathrm{kHz}$. In summary we ignore this effect as whole, assuming that it is small and that it averages out for a sufficiently large data set, since it appears with equal probability for both signs.

In addition to a residual first-order Doppler effect, unmatched wave vectors give rise to a finite momentum transfer to the atoms and a residual recoil shift of

$$
2 \pi \delta \nu_{\text {rec }}=\frac{\hbar|\Delta k|^{2}}{2 m} \approx \frac{\hbar k^{2} \varepsilon^{2}}{2 m}
$$

With the numbers above we obtain $\delta \nu_{r e c}=0.72 \mathrm{~Hz}$ and therefore neglect this shift.

\subsection{Cross-talks between different systematic effects}

The four systematic shifts of the previous sections (CIFODS, second-order Doppler, AC-Stark and pressure shift with parameters $\kappa_{D S}, \kappa_{S O D}, \kappa_{A C}$ and $\kappa_{P S}$ ), that are extrapolated and interpolated by the global minimization of (4.4), are coupled to each other to some extent. In this section we show that these cross-talks are negligible at the current level of accuracy. 
SOD/AC-Stark: Just like the AC-Stark shift, the SOD shift depends slightly on the laser power. This is because an additional $205 \mathrm{~nm}$ photon can ionize the $3 \mathrm{~S}$ level. Slower atoms are more prone to ionization since they stay longer in the laser field. The laser intensity scaling for the AC-Stark shift and ionization is not the same (quadratic vs. cubic). The result is a laser power dependence of the SOD shift. Ignoring this dependence in (4.4) leads to a systematic shift that we estimate by comparing two Monte Carlo simulations using the same set of atomic trajectories. With one, we extrapolate to zero laser power using the global minimization (as in the experiment) with realistic experimental conditions. In the second, we turn off the AC-Stark effect by setting $\beta_{A C}$ in (5.13) and (2.43) to zero. Then we run the same minimization without $\kappa_{A C}$. The frequency difference of the two simulations is less than $3 \mathrm{~Hz}$ for all temperatures between $T=1 \ldots 200 \mathrm{~K}$.

In another simulation we use separate parameters, $\kappa_{A C}$, for different temperatures instead of a global one. Using realistic experimental conditions, but with the chirp parameter set to zero and the nozzle distance fixed to $d=19.1 \mathrm{~mm}$, we find that the resulting differences of the extracted absolute frequencies are below $0.1 \mathrm{~Hz}$ for all nozzle temperatures $T \leq 180 \mathrm{~K}$. With that, we conclude that it is safe to ignore the SOD/AC-Stark cross-talk in our data analysis.

SOD/CIFODS: Faster atoms with a larger SOD shift deposit their fluorescence light further downstream of the atomic beam, which mimics a CIFODS with our detection scheme. This has already been discussed in Fig. 5.2 with the corresponding simulation data and is repeated in Fig. 5.21. As discussed in section 5.1, the compensation of the CIFODS is based on the assumption that it is linear in the chirp parameter $b$ (see (5.6)). Adding a SOD induced spurious offset to the chirp parameter $\Delta b$ does not effect this linearity and leads to exactly the same compensation method (5.7). The unperturbed transition frequency comes out correctly, but the (irrelevant) chirp parameter does not. This assumes that $\Delta b$ is constant across the PCV, a feature that cannot be read from Fig. 5.21. However, we can use it to read off the range of SOD shifts covered by the auxiliary detectors, i.e. $f_{2}-f_{3}$, and multiply by $\kappa_{D S}$ (see (5.6)) to obtain an estimate of the linear SOD induced spurious CIFODS of $400 \mathrm{~Hz} \times 0.043=17 \mathrm{~Hz}$ at $T=7 \mathrm{~K}$. For larger temperatures, the position dependent SOD difference between $f_{2}$ and $f_{3}$ increases linearly, reaching $\approx 74 \mathrm{~Hz}$ at $T=30 \mathrm{~K}$. With regard to Fig. 5.21, we expect the nonlinear contribution to be even smaller.

This cross-talk can again be estimated by selectively turning some effects off in the Monte Carlo simulations and running them with the same set of atomic trajectories. In this case, we turned off the AC-Stark effect and keep the nozzle distance at $d=19.1 \mathrm{~mm}$. We run the simulations with $T=1 \ldots 200 \mathrm{~K}$ and with a range $b=-0.1,0.0,+0.1$ extrapolating to zero temperature. Comparing 


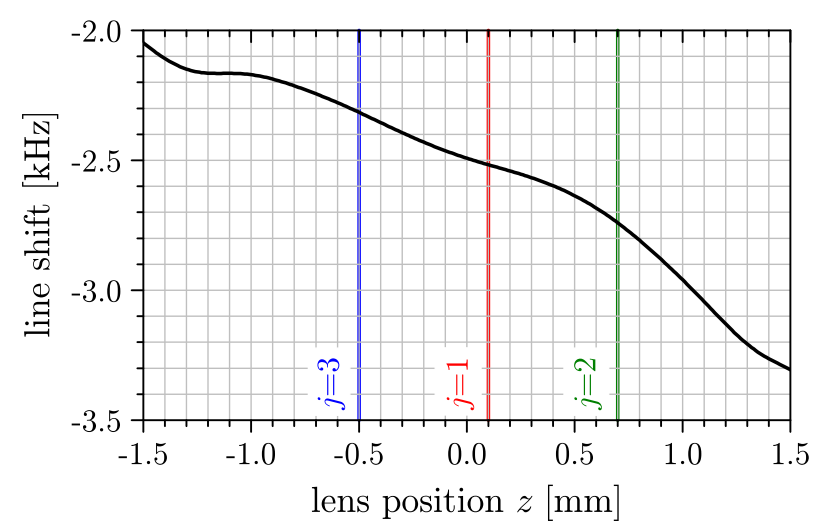

Figure 5.21.: Monte Carlo simulation of the second-order Doppler shift (SOD) that mimics a CIFODS at $T=7 \mathrm{~K}$ without the AC-Stark and pressure shift. This graph repeats the zero chirp $(b=0)$ curve of the right side of Fig. 5.2 at a larger vertical scale. It shows the obtained line center as a function of the position $z$ of the lens that collects the fluorescence signal into a $600 \mu \mathrm{m}$ fiber with respect to the center of the pulse collision volume (PCV)(see also Fig. 3.9). In this plot the atoms travel from left to right. The approximate positions of the three detectors $(j=1,2,3$ in Fig. 3.9) are marked with vertical lines (see also Fig. 5.2). Data with the auxiliary detectors, $j=2$ and $j=3$, show a frequency difference of $\approx 400 \mathrm{~Hz}$.

the $b=0$ value with the $b= \pm 0.1$ interpolated to $b=0$ value effectively turns on and off all SOD/CIFODS cross-talk. The frequency difference was found to be $12 \mathrm{~Hz}$ which is negligible at the current level of uncertainty.

CIFODS/AC-Stark: Similar to the SOD/AC-Stark cross-talk, the CIFODS/ACStark cross-talk results from the fact that slower atoms are more likely to be ionized than faster atoms so that the laser power has an influence on the velocity distribution of the contributing atoms. The velocity distribution in turn is directly connected to any type of Doppler shift and hence also the CIFODS. In this particular case we expect a laser power dependence of the parameters $\alpha_{j}$ in (5.5).

To investigate this cross-talk we compare the extrapolation in power and chirp for different chirp coefficients $(b=-0.1,0,0.1)$ and laser powers $(P=$ $0,50,100,150 \mathrm{~mW})$ with the extrapolation in power when the chirp coefficient is zero. We keep the distance to nozzle and temperature constant $(d=19.1 \mathrm{~mm}$, $T=7 \mathrm{~K}$ ). The resulting difference is only $5 \mathrm{~Hz}$, which is negligible at the current level of uncertainty.

Pressure/CIFODS: Varying the pressure by changing the distance between the nozzle and the PCV, as it is done in the experiment, also changes the divergence (or collimation) of the atomic beam, which in turn varies the CIFODS. This is expressed in approximate form through (5.4). As before, we can use the Monte 
Carlo simulation to model the CIFODS dependence on the atomic beam collimation within the PCV more accurately. However, we do not model the pressure shift with our Monte Carlo code (see section 2.5). Nevertheless, we can get partial information on the pressure/CIFODS cross-talk from it. For this purpose we use again the simulation data generated under realistic experimental conditions and analyze it like the experimental data, but in this case separately for the two nozzle distances $d=19.1 \mathrm{~mm}$ and $d=27.1 \mathrm{~mm}$ and with two separate CIFODS coefficients $\kappa_{D S}$. We then compare the absolute frequencies obtained in this way by an analysis that uses only a common coefficient $\kappa_{D S}$ as was done for the real data analysis. The comparison gives an estimate of the pressure/CIFODS cross-talk assuming that the pressure shift is independent of the atomic beam divergence (see below). We performed these tests at all temperatures used in the experimental data evaluation $(T \leq 30 \mathrm{~K})$ and obtain typical differences between the sets of $\approx 4 \mathrm{~Hz}(T=7 \mathrm{~K}), \approx 8 \mathrm{~Hz}$ $(T=15 \mathrm{~K})$ and $\approx 24 \mathrm{~Hz}(T=30 \mathrm{~K})$. This is negligible at the current level of uncertainty.

Pressure/SOD: There are several physical mechanisms that leads to a cross-talk between the SOD and the pressure shift. Since the SOD is mostly determined by the temperature $T$ of the nozzle, these would be a temperature dependence of the pressure shift, or a temperature dependence of how we measure the pressure shift. The first mechanism is due to the $1 / \sqrt{T}$ dependence of the number density $n$ of particles in the beam. This is because we keep the total flux into the nozzle constant but their escape velocity is proportional to $1 / \sqrt{T}$. Second, the cross-section of the collisional shift and collision rate, depends on velocity. Third, the ratio of atomic vs. molecular hydrogen in the beam, i.e. the recombination rate, depends on temperature (see Fig. 3.8). This changes the chemical composition of the beam. The fourth mechanism is a cross-talk between SOD shift and beam divergence, which mimics an intra-beam pressure shift with our measurement procedure based on the variation of the distance between the nozzle and the PCV.

The temperature dependence of the collisional shift is $\propto T^{-1 / 5}$ as obtained from (5.20) with $n \propto 1 / \sqrt{T}$. The mean pressure shift is estimated to be $0.87 \mathrm{kHz}$ (see section 5.4). Using the uncertainties of the absolute frequency for the various nozzle temperatures as a weight, we compute the standard deviation of $\delta \nu_{P S}$ to $52 \mathrm{~Hz}$. This is an error that we introduce in our data analysis by assuming that the pressure shift coefficient $\kappa_{P S}$ is independent of temperature.

The cross-talk between the SOD and the beam divergence is actually not due to the pressure shift itself but is rooted in the way we measure the pressure shift. Therefore we can use our Monte Carlos simulations to evaluate it, even though it does not describe the pressure shift. For this purpose we are comparing the results from two Monte Carlo runs that are obtained with two nozzle distances 
and with $b=0$ (no CIFODS) as well as the AC-Stark shift turned off. Due to its large aspect ratio, atoms that cross the PCV under a large angle spend less time within it and, hence, the signal from the faster atoms along these trajectories is relatively enhanced. The faster atoms in turn experiences a larger SOD. The absolute frequencies deviate by only $1 \mathrm{~Hz}$, which is negligible at the current level of uncertainty.

The evaluation of the temperature dependence of the degree of dissociation is difficult. According to our estimation (see section 5.4), the van-der-Waals coefficient for atom-molecule collisions is of the same order of magnitude as for atom-atom collisions. However, the presence of the quadrupole electric field of the molecule decreases the pressure shift because it rather quenches the 3S state in hard collisions. Therefore, the shift from collisions with hydrogen molecules is smaller than from collisions with atoms, and we neglect this mechanism. This suggests that even if the degree of dissociation changes with temperature, the dependence of the pressure shift is small.

We can verify all of these effects and all other temperature couplings to the absolute frequency to some extent using the experimental data. For this purpose we perform the global minimization of (4.4) for each nozzle temperatures separately ignoring the SOD, i.e. by setting $\kappa_{S O D}=0$. The CIFODS, AC-Stark shift and pressure shift are still compensated in this way but all temperaturedependent couplings are removed because the temperature is fixed. Finally, we extrapolate these results to zero temperature, and find that this result deviates from the global fit (4.3) by $192 \mathrm{~Hz}$ only.

Pressure/AC-Stark: There are two mechanisms that cause a cross-talk between the AC-Stark shift and the pressure shift. Again, the collimation of the atomic trajectories that transverse the PCV changes with the nozzle distance. This collimation possibly has an influence on the pressure shift giving rise to a contribution that is nonlinear in the atomic density. At the same time a larger divergence leads to a relative increase of the number of atoms that sample the lower intensity radial fringes of the PCV. Secondly, ionization preferentially effects atoms that travel closer to the optical axis where the density is higher. The pressure shift is the only systematics discussed in this work that is not included in our Monte Carlo simulation. Therefore we cannot estimate this cross talk in this way. However, we assume that this cross-talk contributes only a small correction and neglect it.

It may seem conceivable that the normalization of the Doppler-free signal with the Doppler-broadened signal (see sections 5.5 and 4.2), does not fully remove the laser power, $P_{i}$, dependence of the normalized line amplitudes, $A_{i}$, for whatever reason. In that case the parameters $\kappa_{A C}$ and $\kappa_{P S}$ in (4.3) are not linearly independent. This might be suspected to explain the discrepancy between the experimentally determined AC-Stark shift and the theoretical 
value as described in section 5.2. To investigate this scenario, we have rerun the data evaluation fixing $\kappa_{P S}=0$ and find for the AC-Stark coefficient $\kappa_{A C}=136.73 \mathrm{~Hz} / \mu \mathrm{W}$. This is compatible with the value from the global minimization using all parameters (see table 4.1).

Estimating the cross-talk systematic shifts one-by-one has the advantage that on can identify the largest contribution. After convincing ourselves that the items above are small individually, we can treat them all together in a Monte Carlo simulation (see next section). In this way we also include more complex cross-talks together with line distortion effects (see previous sections). For this purpose we evaluate simulation results obtained with realistic experimental conditions exactly like the real experimental data. We obtain a deviation of only $24 \mathrm{~Hz}$ from the set frequency that enters the simulation code. This "fitting model" uncertainty is included in the error budget in table 5.1. We believe that this characterizes all systematic shift that have been discussed so far, except for the pressure shift, which was estimated independently. The purpose of listing the cross-talks above is to exclude possible large and compensating systematic shifts that would require a much more careful estimation.

\subsection{Results and Error Budget}

The most straightforward, but not very transparent way to determine the overall uncertainty, is to generate an artificial data set with the Monte Carlo simulations described in the previous section, and analyse it in precisely the same the way as the experimental data. Except for the pressure shift, we can include all systematic effects in these simulations and vary the parameters such as the laser power and the chirp parameter in accordance with the experimental variation. In doing so, we find a total fitting model induced systematic shift of $24 \mathrm{~Hz}$ (see also section 5.11) which we include as an uncertainty into the error budget.

As detailed in section 4.4, we analyze the experimental data with a global minimization of (4.4) parameterizing the unperturbed transition frequency $f_{0}$ and find for the hyperfine centroid of the 1S-3S transition:

$$
f_{1 \mathrm{~S}-3 \mathrm{~S}}(\text { centroid })=2922743278665.79(72) \mathrm{kHz} .
$$

Most of the systematic shifts are taken into account in the global minimization by coefficients for the CIFODS, the second-order Doppler, the AC-Stark and the pressure shift $\kappa_{D S}, \kappa_{S O D}, \kappa_{A C}$ and $\kappa_{P S}$ respectively. With this minimization we extrapolate and interpolate with noisy experimental data, so that the resulting uncertainty should be treated as a statistical uncertainty. In addition to this common estimate, we wish to obtain separate values for the systematic shifts - for example to determine the best route for future improvements. We have already addressed some aspects of individual systematics in the discussions of section 5 . 


\begin{tabular}{||l|c|c|c|c||}
\hline contribution & section & average effect & correction & uncertainty \\
\hline \hline statistics & 4.4 & - & - & 0.11 \\
\hline CIFODS & 5.1 & +0.79 & - & $0.08^{*}$ \\
\hline SOD & 5.3 & -3.20 & - & $0.26^{*}$ \\
\hline AC-Stark & 5.2 & +4.60 & - & $0.30^{*}$ \\
\hline residual Doppler & 5.4 & +0.93 & - & $0.30^{*}$ \\
\hline background pressure & 5.10 & - & - & $0.48^{*}$ \\
\hline DC-Stark & 5.4 & - & - & 0.09 \\
\hline Zeeman shift & 5.5 & +0.031 & -0.031 & 0.015 \\
\hline quantum interference & 5.6 & -0.002 & +0.002 & 0.002 \\
\hline incoherent line pulling & 5.8 & -0.25 & +0.25 & 0.050 \\
\hline multi-parameter CIFODS & 5.9 & -0.05 & +0.05 & 0.01 \\
\hline fitting model & 5.1 & - & - & 0.10 \\
\hline maser & 3.1 & -0.30 & +0.30 & 0.030 \\
\hline \hline total & & & +0.57 & 0.72 \\
\hline
\end{tabular}

Table 5.1.: Error budget of the $1 \mathrm{~S}(F=1)-3 \mathrm{~S}(F=1)$ measurement. All values in $\mathrm{kHz}$. "Average effect" is the weighted mean calculated using (5.49). The uncertainties marked with * have been determined through (5.50). Adding the first 5 rows of the last column quadratically results in $0.52 \mathrm{kHz}$, which agrees well with the uncertainty of $f_{0}$ given in table 4.1. The uncertainty of the nonlinear contribution of the second-order Doppler (SOD) shift is taken into account by increasing the experimental error bars with the $q=2 \ldots 4$ model uncertainties as described in section 5.3. We add all uncertainties in this table quadratically to obtain the final uncertainty of $0.72 \mathrm{kHz}$.

To compile meaningful and separate values for the systematic that enter (4.3), we use the weighted mean of the corresponding linear correction:

$$
\langle\Delta f\rangle=\frac{\kappa_{x} \sum_{i} x_{i} / \sigma_{1, i}^{2}}{\sum_{i} 1 / \sigma_{1, i}^{2}}
$$

with the corresponding $\kappa_{x}$ parameter and the variance $\sigma_{1, i}^{2}$ of the frequency $f_{1, i}$. Here $x$ stands for any of the variables $\beta, T, P$ or $A$, where these variables are the frequency difference obtained with detectors $j=2$ and $j=3$, the nozzle temperature, the laser power and the line amplitude respectively (see section 4.4). The average uncertainty the frequency correction can be calculated from the uncertainty of the corresponding parameter $\kappa_{x}$

$$
\sigma_{\langle\Delta f\rangle}=\left|\frac{\sigma_{\kappa_{x}}\langle\Delta f\rangle}{\kappa_{x}}\right|
$$

Individual contributions are presented in table 5.1. 


\section{Conclusions and Outlook}

\subsection{Discussion of Measurement Results}

In the course of this work, a high precision measurement of the $1 \mathrm{~S}-3 \mathrm{~S}$ transition (hyperfine component $F=1 \rightarrow F=1$ ) has been performed. All corrections and the error budget is given in table 5.1. After applying them to the mean transition frequency, we obtain the unperturbed absolute frequency:

$$
f_{1 \mathrm{~S}-3 \mathrm{~S}}(F=1)=2922742936716.72(72) \mathrm{kHz}
$$

Subtracting the hyperfine shifts of -341949069.6(8) Hz [10], gives the hyperfine centroid:

$$
f_{1 \mathrm{~S}-3 \mathrm{~S}}(\text { centroid })=2922743278665.79(72) \mathrm{kHz}
$$

It is in good agreement but more than 20 times more accurate than our previous room temperature measurement [21]. It is 3.5 times more accurate than the previous most precise 1S-3S measurement [7] (LKB, France) and disagrees by 2.1 combined standard deviations from it. Due to the improved uncertainty of our measurement, the combined standard deviation is almost entirely given by the uncertainty in [7]. Since both experiments measure the same transition a discrepancy can not be due to the theory. The probability (assuming Gaussian distributions) to find a discrepancy of $2.1 \sigma$ is $3.4 \%$. One may also think of some possible under-, overestimated or unexplored systematics in either one of experiments.

At the same time, we can use our result, the measured $1 \mathrm{~S}-2 \mathrm{~S}$ transition frequency [9], and QED in form of (1.4) to obtain a value for the Rydberg constant, which is twice as accurate as with all so far available hydrogen spectroscopy data combined.

$$
R_{\infty}=10973731.568226(38) \mathrm{m}^{-1},
$$

and an independent value of the RMS proton charge radius

$$
r_{p}=0.8482(38) \mathrm{fm}
$$

In doing so, we have used the collection of terms and the values for the fine structure constant, as well as the electron-to-proton mass ratio given in the most recent published CODATA assembly [4]. More accurate values of these constants are available [8], but that has no influence on the results presented here. Our value for the Rydberg constant is more accurate than the most recent published CODATA value, but differs by 3.7 combined standard deviations from it. The proton charge radius 
disagrees by 2.9 combined standard deviations with the hydrogen world data as of 2014 , but is in better agreement with the most recent muonic value [16] and a recent measurement of the Lamb shift [6].

We have put our most effort to minimize the largest systematic effects as much as possible. To this end the measurement has been performed at cryogenic temperatures and this work constitutes the first cryogenic measurement of the 1S-3S transition in hydrogen. This reduces the residual first order Doppler shift $(0.79 \mathrm{kHz}$, CIFODS). We have studied this new frequency comb spectroscopy inherent effect both experimentally and theoretically. Simple and intuitive formulas have been derived and comprehensive Monte Carlo simulations performed. We found excellent agreement between theory and experiment (see section 5.1). In order to measure it experimentally, we have developed a flexible detector design, which upon the change of a single fiber can significantly change its spatial detection profile and thus its sensitivity to the chirp induced residual Doppler shift. Using several different detectors, the chirp induced first order Doppler shift can be minimized and measured at the same time. We consider this method as the first step to fully spatially resolved frequency comb spectroscopy, which we plan to extend with a single photon EMCCD camera. Such a technique, where the transition frequency is resolved for different points in space, would lead to better understanding and instantaneous characterization of several important effects.

One of the most troubling systematic shifts in high precision spectroscopy experiments is the second order Doppler shift (SOD) due to the unknown velocity distribution, which is experimentally difficult to access. With a cryogenic atomic beam also this effect could be reduced to $-3.19 \mathrm{kHz}$ only. To characterize the second order Doppler shift, measurements at seven different temperatures from $4.5 \mathrm{~K}$ to $180 \mathrm{~K}$ have been performed. The temperature dependence has been analyzed with Maxwell-like velocity distributions by performing Monte Carlo simulations. It was found that a simple linear dependence describes the data best, despite some predicted non-linearity at higher temperatures, if the Maxwellian velocity distribution is assumed. We believe that this shows the deviation from the Maxwellian distribution in atomic beams at low temperatures, such that the low velocity tail is suppressed. This data can be used in future experiments, to understand the velocity distribution, which inevitably limits the precision, when using atomic beams. Despite excellent agreement with the data, only temperatures below $30 \mathrm{~K}$ have been used for final evaluation, in order to minimize any possible remaining non-linearities (see section $5.3)$.

The collisional shift often constitutes only a small correction on the order of $100 \mathrm{~Hz}$, but is typically very difficult to measure experimentally. Therefore, it is often only estimated theoretically and corrected. However, many uncertainties, such as gas composition, exact pressure and its inhomogeneities within the interaction region, have to be assumed. Therefore, despite an almost two-fold increase of the final uncertainty, we performed a direct measurement of this effect by varying the distance between the interaction region and the hydrogen nozzle (see section 5.4). 
This was possible due to a unique feature of the direct frequency comb spectroscopy, which limits the interaction region to a tiny volume, in which the pulses overlap.

The AC stark shift constitutes the largest systematic effect in our experiment (4.59 kHz on average). It has been measured experimentally by varying the intracavity laser power and extrapolating in the transmitted power. Nonlinearity is shown to be negligible (see appendix B). By performing several different separate measurements of the cavity waist and output mirror transmittivity (see. appendix A) and using Monte Carlo simulations, we obtained an AC Stark shift coefficient, which is about 3.5 times smaller than the experimental one. Some possible mistake in the determination of the waist or transmittivity of the mirror can possibly explain this discrepancy, since the cavity is operated close to the instability region where the waist is difficult to measure. The mirrors are operated in vacuum with UV light and are constantly flushed with oxygen, which also can influence the transmission measurement. Also some undiscovered mistake in the calculation could be the cause. This is the major unknown of this measurement and needs further investigation.

We would like to stress that all systematic shifts, which we are aware of, are significantly smaller than the Proton Radius Puzzle discrepancy (7 kHz for $1 \mathrm{~S}-3 \mathrm{~S}$ ), which together with the result by our competitors at LKB [7], makes the 1S-3S measurements in hydrogen a unique test of the Proton Radius Puzzle. All significant effects of this experiment have been measured experimentally using a simple interpolation or extrapolation, which is a very robust and simulation-independent method, as long as nonlinear terms can be shown to be negligible, which is extremely well justified for the parameters of this experiment.

Two new experiments, which have been performed in our group are currently under evaluation. The 1S-3S centroid deuterium transition, using the same setup as for this work, has the advantage of a significantly smaller AC Stark shift and two times smaller second order Doppler shift. The measurement was done during the time of this work. The 2S-6P transition in hydrogen has been recently measured in our group with sub $\mathrm{kHz}$ precision and is currently being analyzed and prepared for publication. The corresponding $2 \mathrm{~S}-6 \mathrm{P}$ measurement in deuterium is under preparation. Exciting contributions to the Proton Radius Puzzle and significant improvement of the Rydberg constant and the RMS proton charge radius are expected from these experiments.

\subsection{Frequency Comb Spectroscopy Technique Investigation Results}

In the last two decades stabilized frequency combs became the standard tool for absolute frequency determination in most precision labs around the globe [22], replacing the tedious operation of long phase locked frequency chains. Their simplicity and broad wavelength coverage has been appreciated throughout the spectroscopy community and beyond it. T.W. Hänsch and J.L. Hall were honored in 2005 with 


\section{Conclusions and Outlook}

the noble prize for their crucial contribution to this fundamentally important development. Frequency combs found also numerous applications besides the absolute frequency determination [23], [24]. The usage of frequency combs directly as excitation source for two-photon transitions, despite being one of the original ideas behind the frequency comb [25], have not so far been applied in many precision spectroscopy experiments.

This work is the first experimental demonstration of the Two-photon Direct Frequency Comb Spectroscopy below $1 \mathrm{kHz}$ level [43, 80-83]. It shows that this technique can be as precise as CW laser spectroscopy, offering several important advantages, which however come at a cost of a specific systematic residual Doppler shift. In this work, we have carefully analyzed and described the advantages and disadvantages of this technique, its inherent systematic shifts and offered a technique to handle them. The most apparent advantage is the possibility of generating UV and DUV laser sources through harmonics generation in crystals and gas targets, where CW lasers with sufficient power are not available yet. This paves the way to precision experiments with hydrogen-like ions, as for example anticipated in our group with the $\mathrm{He}^{+}$spectroscopy [19] and many others. The confinement of the Dopplerfree interaction region to a tiny volume with the size of the pulse length and the waist of the beam, allows for separate Doppler-broadened fluorescence collection. We have shown that this can be used as efficient normalization signal, such that almost shot noise limited statistics can be reached, despite in this wavelength region often unavoidable laser power fluctuations as well as atomic flux fluctuations. The small volume is further suitable for efficient light collection, efficient screenings of electric and magnetic fields. Pressure and field inhomogeneities are typically smaller and can be measured through a simple variation of the distance between the atomic source and the pulse collision volume. The opposite side of an inherently confined interaction region is a reduced overall count rate and time-of-flight broadening, such that only at the center of the pulse collision volume and for very slow atoms, the same count rate as with a $\mathrm{CW}$ laser with the same average power and beam size can be reached. It can not be simply increased by collecting light from a larger part of the interaction region. The chirp induced first order Doppler shift has been discovered in the course of this work [21] and was the limiting systematics for our previous room temperature measurement. Its theory and comparison with experiment is discussed in this work. A robust measurement method was developed, such that this new systematic frequency shift does not limit the precision and is not expected to be the next largest systematics. Another important fundamental systematic shift, which can appear in both CW and frequency comb two-photon spectroscopy, which we refer to as Tilted Wave Front Doppler Shift is discussed in this work in section 5.10. It explains the question, why the first order Doppler shift, despite cavity imperfections, is strongly suppressed in two-photon spectroscopy. To our knowledge, this is the first detailed description of this effect. 


\subsection{Suggestions for Future Improvements}

The current 1S-3S measurement in hydrogen at the sub kilohertz level resolves the line width to only slightly more than $10^{-3}$, which is a quite moderate value, as compared for instance to the $2 \mathrm{~S}-4 \mathrm{P}$ measurement [5], where the resolution is pushed to $10^{-4}$ of the line width. The relatively narrow $1 \mathrm{MHz}$ natural line width of the $1 \mathrm{~S}-$ $3 \mathrm{~S}$ transition makes it very attractive for precision measurements (compare $20 \mathrm{MHz}$ line width of the one-photon $2 \mathrm{~S}-4 \mathrm{P}$ transition). Due to the cryogenic atomic beam and optimized design, many important systematic shifts such as the second order Doppler shift and CIFODS are reduced. Other systematic shifts are under sufficient control and can be efficiently measured and characterized. A comprehensive Monte Carlos simulation has been developed by Arthur Matveev in our group, which can analyze in detail the experiment and systematic shifts. We believe therefore that it is not unreasonable to push the limit of the uncertainty an order of magnitude further to the $100 \mathrm{~Hz}$ level, reaching the uncertainty for the RMS proton charge radius, as given by the muonic hydrogen spectroscopy [3]. This is a challenging and fascinating goal. Suggestions on how the experiment can be improved are sketched in this section.

By looking at the error budget table 5.1 the AC Stark shift is identified as the largest effect in this experiment. It is on average $4.59 \mathrm{kHz}$ (determined to $6.5 \%$ ). Assuming same ratio of uncertainty to correction, one needs to reduce the AC Stark shift by about a factor of five to come well below the $100 \mathrm{~Hz}$ level. With a larger laser power, the waist size could be increased by the same factor without losing signal, if one takes into account the larger number of interogated atoms. To increase the laser power by a factor of five is a very challenging task. As explained in section 3 commercial titanium:tapphire lasers operate at repetition rates around $80 \mathrm{MHz}$, such that we are forced to filter out every second comb mode by the SHG cavities, in order to prevent incoherent line pulling (see section 5.9). We suggest to shorten the laser cavity by a factor of two, while exchanging the active mode-locker (acoustooptic modulator) with one, which has its resonance frequency at $157.6 \mathrm{MHz}$. This would increase the effective laser power at $820 \mathrm{~nm}$ by a factor of two and the fourth harmonic at $205 \mathrm{~nm}$ by a factor of four (first SHG cavity saturated, thus only linear increase, second SHG quadratic). Another possibility to minimize the AC Stark shift, is to use an additional laser at a wavelength, where the AC Stark shift flips the sign (NIR region). The overall shift would be decreased, which still would need to be measured. However, in order for such a compensation to function, the spatial overlap as well as intensity match within the entire interaction region between the excitation laser and the compensating laser needs to be guaranteed. We believe, that this is a very difficult task to achieve. Another way to increase the fundamental power at $820 \mathrm{~nm}$, is to use and external multi-pass titanium:sapphire amplifier.

In the current setup, Balmer- $\alpha$ fluorescence photons are collected with lenses and multi-mode fibers and then guided to photo-multiplying tubes (Hamamatsu, H7421). The quantum efficiency at this wavelength is about $20 \%$ with $5 \mathrm{~mm} \times 5 \mathrm{~mm}$ active 
sensor area. We suggest instead to use a single photon EMCCD camera (electron multiplying CCD, e.g. Andor Ixon 888), which exhibits $95 \%$ quantum efficiency and $10 \mathrm{~mm} \times 10 \mathrm{~mm}$ sensor area. With this camera the total light collection efficiency can be improved by a factor of 10 (5x higher QE and $2 x$ larger solid angle). Further, the need for several separate detectors for CIFODS compensation is omitted, as each pixel of the camera is essentially a separate detector. In order to keep the signal to noise ratio (dominated by dark current and shot noise) at the current level of $\mathrm{S} / \mathrm{N}=$ 10 , the $1024 \times 1024$ pixels need to be binned to $10 \times 10$ super pixels. We note, that it is not a trivial task, to design an objective with both a large solid angle and sufficient resolution and image depth. Compromises will need be made.

In the current setup, the atomic beam propagates along the laser beam axis, which reduces the time-of-flight broadening (PCV $600 \mu \mathrm{m}$ long, while only $160 \mu \mathrm{m}$ in diameter), such that almost natural line width is obtained for low temperatures $(4.5 \mathrm{~K}$ and $7 \mathrm{~K})$. However, collinear propagation of the atomic and the laser beams, necessitates a T-shaped nozzle (see fig. 3.1). Lothar Maisenbacher has performed simulations, which showed, that in such a nozzle, hydrogen atoms collide few tens to hundreds times with the walls, where recombination with other hydrogen atoms occurs. From our data (see section 3, fig. 3.8) we can estimate our dissociation ratio to around $10 \%$. With an atomic beam propagating orthogonally to the laser beam, the number of wall collisions can be significantly reduced [54, 84], thus increasing the dissociation ratio and the amount of hydrogen atoms in the beam. With less collisions however, also the velocity distribution could change. If at the same time the waist is increased by roughly a factor of five (in order to reduce the AC Stark shift), the narrow line width can be preserved.

The second order Doppler shift is the second largest effect for this measurement $(-3.19(26) \mathrm{kHz})$. Currently most data is measured at $7 \mathrm{~K}$, while some data has been taken at $4.5 \mathrm{~K}$. With a suitable cryostat and thermal radiation shielding temperatures at the level of 1-2 K can be reached, reducing the SOD shift to 300-600 Hz. However, as seen in figure 3.8 the count rate decreases very strongly at temperatures below $\approx 30 \mathrm{~K}$. This is because a thick layer of frozen molecular hydrogen forms inside the nozzle that atomic hydrogen diffuses into. The adsorption energy has been measured to $k_{B} \times 38(5) \mathrm{K}$ [84]. Once trapped inside the layer, atomic hydrogen recombines very efficiently to molecules and the count rate drops. Though the actual count rate at such a temperature needs to be measured, a dramatic decrease is expected. With a proper nozzle for orthogonal atomic beam propagation this problem may be reduced. The necessity of lower temperatures underlines once again the need of better light collection and dissociation ratio increase. Further improvement of the second order Doppler shift uncertainty can be made, if the velocity distribution is characterized as was for instance recently done in [62]. In our opinion, the second order Doppler shift is the most challenging systematics for an anticipated improvement of the $1 \mathrm{~S}-3 \mathrm{~S}$ measurement. 


\section{Appendix}

\section{A. Enhancement Cavity Characterization}

\section{A.1. Transmission Measurement}

In this chapter the characterization of the enhancement cavity of the 1S-3S hydrogen experiment is presented and discussed. This is particularly important, since many systematic shifts depend on the internal circulating power and the waist size of the cavity mode. In particular a comparison of the extrapolated AC Stark shift with simulation is only possible if the transmission and waist size are known. The tilted wave front Doppler shift is estimated relying on the knowledge of the mode structure and waist. Other effects such as second order Doppler shift also slightly depend on the waist size and power, though these couplings have been found negligible at the current level of uncertainty.

Figure A.1 shows our linear two-mirror enhancement cavity. The length is fixed at $0.9511 \mathrm{~m}$ by the repetition rate of the ps-comb $(157.6 \mathrm{MHz})$. The input coupling mirror $M_{1}$ is mounted on a piezo transducer to lock the cavity using the dither lock technique. The radii of curvature of both mirrors are specified by the manufacturer (Laseroptik) to ROC $=500(25) \mathrm{mm}$. The input coupler $M_{1}$ has a transmission of $T_{1}=6.4 \%$ and a reflectivity of around $R_{1}=90 \%$ while the backside mirror $M_{2}$ has a reflectivity of $R_{2}=97 \%$ with a very small transmission.

To measure the transmission of the backside mirror $M_{2}$ we used the $205 \mathrm{~nm}$ light of the ps-comb. We are interested in the total transmission through the mirror, $\mathrm{MgF}_{2}$ window (5 mm thickness), HR mirror (205 nm, 45, plane) and $90 \% \mathrm{PR}$ mirror (205 nm, $5^{\circ}$, plane), which guide the transmitted light onto the power meter, since from the total transmission and the power recordings the circulating power can be deduced. The resulting value is $T_{t o t}=0.00054(10)$. The uncertainty is estimated from the calibration uncertainty of the power meter (Thorlabs S120VC, 7\% at each power range, totaling 14\%) and statistical uncertainty $6 \%$. We have used two more power meters to confirm this measurement. We have varied the polarization and found no polarization dependence. We also increased the impinging intensity on the mirror by means of an additional lens to verify intensity independent transmission. In this way we increased the intensity by about a factor of $15(10 \mathrm{~mW}$ and $400 \mu \mathrm{m}$ vs. $10 \mathrm{~mW}$ and $100 \mu \mathrm{m}$ ) making it comparable to the intensity, when the cavity is locked. We have measured the transmission of the mirror when the chamber 


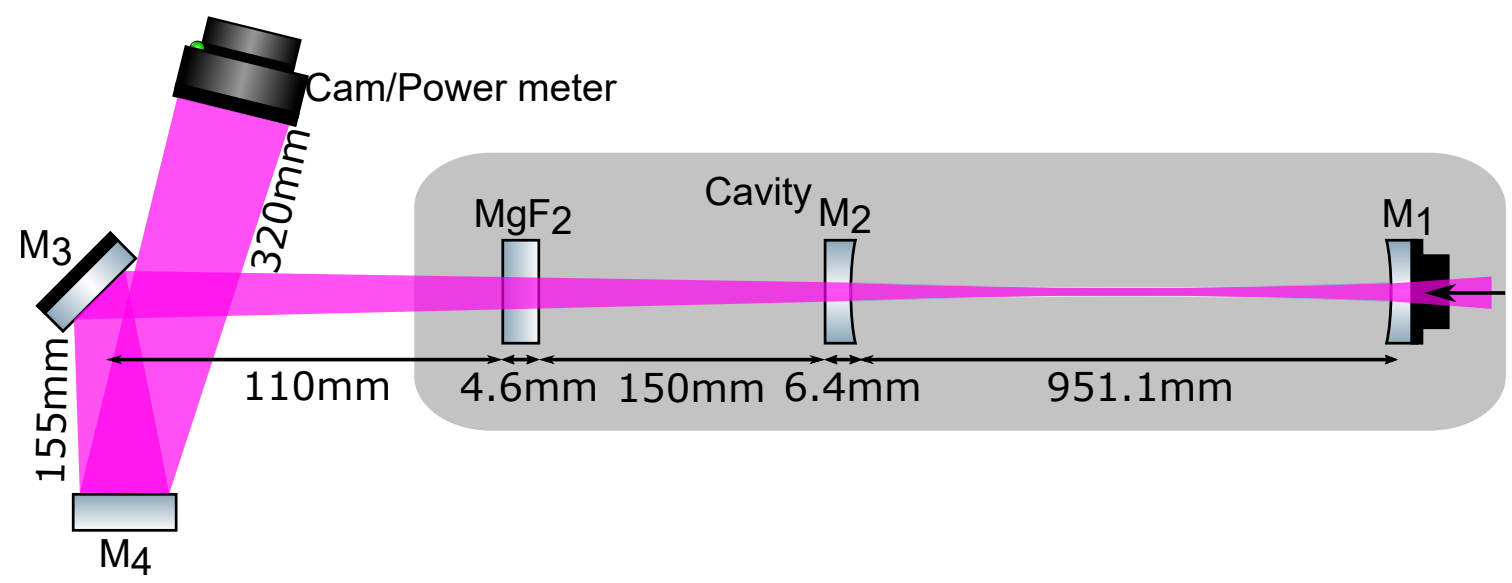

Figure A.1.: A scheme of the UV enhancement cavity of the 1S-3S experiment together with a beam profiler/power meter to measure the intensity profile of the transmitted beam and the transmission of the output coupling mirror is shown (cf. fig. 3.1 in section 3) The two-mirror linear cavity and the $\mathrm{MgF}_{2}$ window are in vacuum. HR mirror $M_{3}(205 \mathrm{~nm}$, $45^{\circ}$, plane) and $90 \% \mathrm{PR}$ mirror $M_{4}\left(205 \mathrm{~nm}, 5^{\circ}\right.$, plane $)$ guide the transmitted light onto the cam/power meter. A $90 \%$ reflecting mirror is used to transmit some light for the dither lock.

is evacuated and the mirror is flushed with oxygen as was done during the $1 \mathrm{~S}$ $3 \mathrm{~S}$ measurement campaign as well as in atmosphere. This is important since the backside $\mathrm{MgF}_{2}$ window is not flushed with oxygen and could possibly degrade over time. The transmission has been measured at different positions of the mirror. In all these tests the transmission was found to be very stably $T_{\text {tot }}=0.00054(10)$.

\section{A.2. Cavity Waist Measurement and Mode Matching}

Since the cavity is close to the stability edge, the waist size depends critically on the radii of curvature of the two mirrors. Therefore we did not want to fully rely on the specified values. To measure the radii of curvature of the two cavity mirrors $M_{1}$ and $M_{2} 3$ different methods have been applied. First we have measured the beam profile outside of the cavity as shown in figure A.1 (WinCamD-LCM-UV, pixel size $5.5 \mu \mathrm{m}, 2 \times 2$ effective binning, $1024 \times 1024$ pixel). The cavity was locked and the chamber evacuated. On the left of figure A.2 the two-dimensional intensity profile is shown. The upper right plot (blue) shows the average intensity along the $x$-direction by summing up all $y$-values at each $x$ position. The red curve is the corresponding Gaussian fit (beam radius at camera position $w_{x}=1.344 \mathrm{~mm}, I(x)=$ $A+B \exp \left(-2\left(x-x_{0}\right) / w_{x}^{2}\right)$. The lower right plot (blue) is the average intensity along the $y$-direction with its Gaussian fit (red, $w_{y}=1.360 \mathrm{~mm}$ ). The complete twodimensional intensity profile has been also fitted with a two-dimensional Gaussian $\left(I(x)=A+B \exp \left(-2\left(x-x_{0}\right) / w_{x}^{2}-2\left(y-y_{0}\right) / w_{y}^{2}\right)\right)$ (elasticity 0.99$)$, resulting in 
following waists $w_{x}=1.328 \mathrm{~mm}$ and $w_{y}=1.333 \mathrm{~mm}$. For the calculation of the radius of curvature we use the mean value of the two-dimensional fit ( $w=1.330 \mathrm{~mm})$.
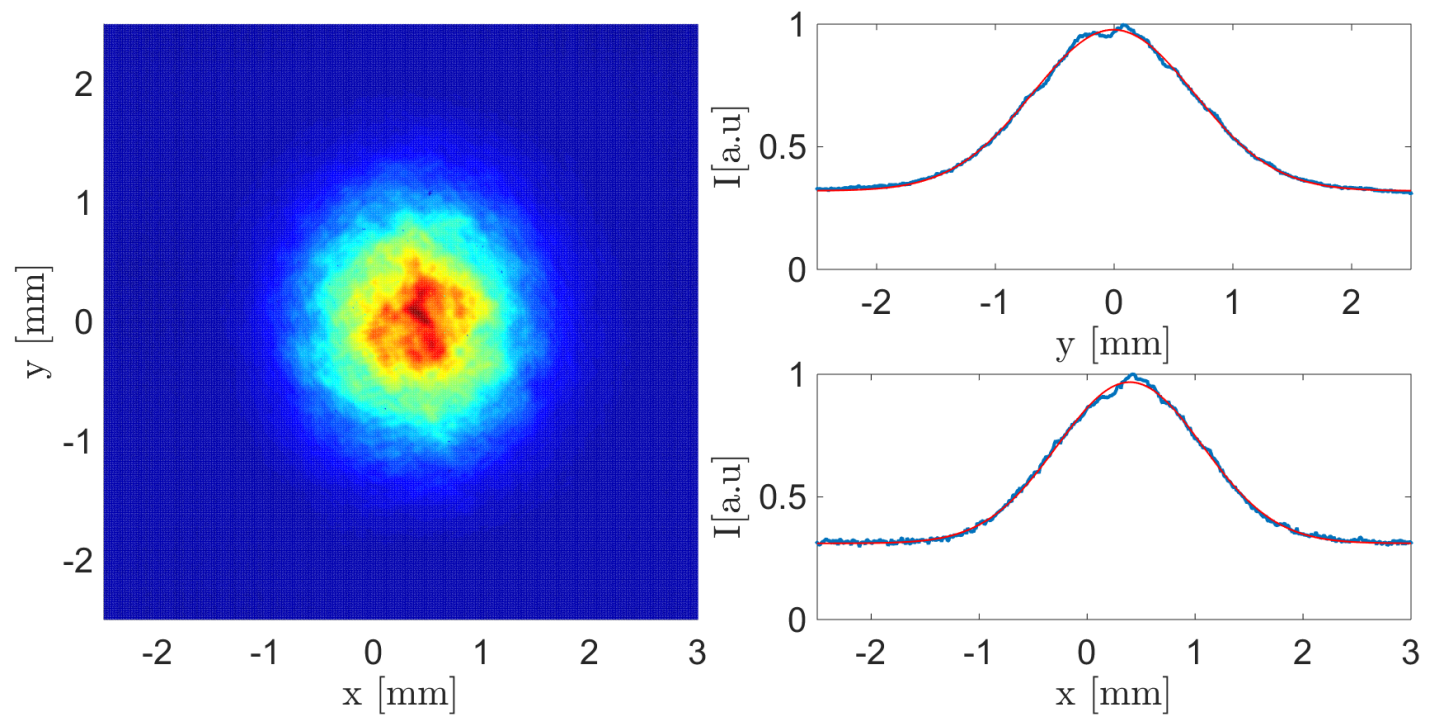

Figure A.2.: On the left the beam profile (WinCamD-LCM-UV, pixel size $5.5 \mu \mathrm{m}, 2 \times 2$ effective binning, $1024 \times 1024$ pixel) is shown. Figure A.1 shows the position of the camera. The upper right plot (blue) shows the average intensity along the $x$-direction by summing up all $y$-values at each $x$ position. The red curve is the corresponding Gaussian fit (beam radius at camera position $w_{x}=1.344 \mathrm{~mm}$ ). The lower right plot (blue) is the average intensity along the $y$-direction with its Gaussian fit (red, $w_{y}=1.360 \mathrm{~mm}$ ). The twodimensional intensity profile has been also fitted with a two-dimensional Gaussian, resulting in $w_{x}=1.328 \mathrm{~mm}$ and $w_{y}=1.333 \mathrm{~mm}$.

We assume that the radii of the two cavity mirrors are equal $R_{1}=R_{2} \equiv R$, which is well supported by white light interferometry presented below. Using the relationship between cavity waist and radius of curvauture, we are left with one unknown $R$ only.

$$
w_{0}=\sqrt{\frac{\lambda}{2 \pi} \sqrt{d(2 R-d)}}
$$

We propagate the beam from the middle of the cavity to the camera using Gaussian matrix calculus and obtain from with the measured beam profile the radius of curvature of $R=495 \mathrm{~mm}$, corresponding to a waist of $w=79 \mu \mathrm{m}$.

One can also extract the radius of curvature from the frequency separation of higher Gaussian-Hermite modes of the cavity. To this end we recorded the transmitted power while scanning the length of the cavity. The left plot in figure A.3 shows the transmitted power scan (blue) with the correponding fit using a sum of eight Lorentzians (red). On the right side of figure A.3 the reflected power scan is displayed. From the separation between modes relative to the free spectral Range (FSR) the radius of curvature of the cavity mirrors can be calculated. The result is 
$R=500(4) \mathrm{mm}$ or $w=84(4) \mu \mathrm{m}$. From the ratio of the amplitude of the TEM00 mode to the sum of all mode amplitudes within one FSR the mode matching can be obtained. This results in $30.3 \%$ mode matching. From the drop of the TEM00 mode a more reliable value of $28.8 \%$ for the mode matching is obtained.
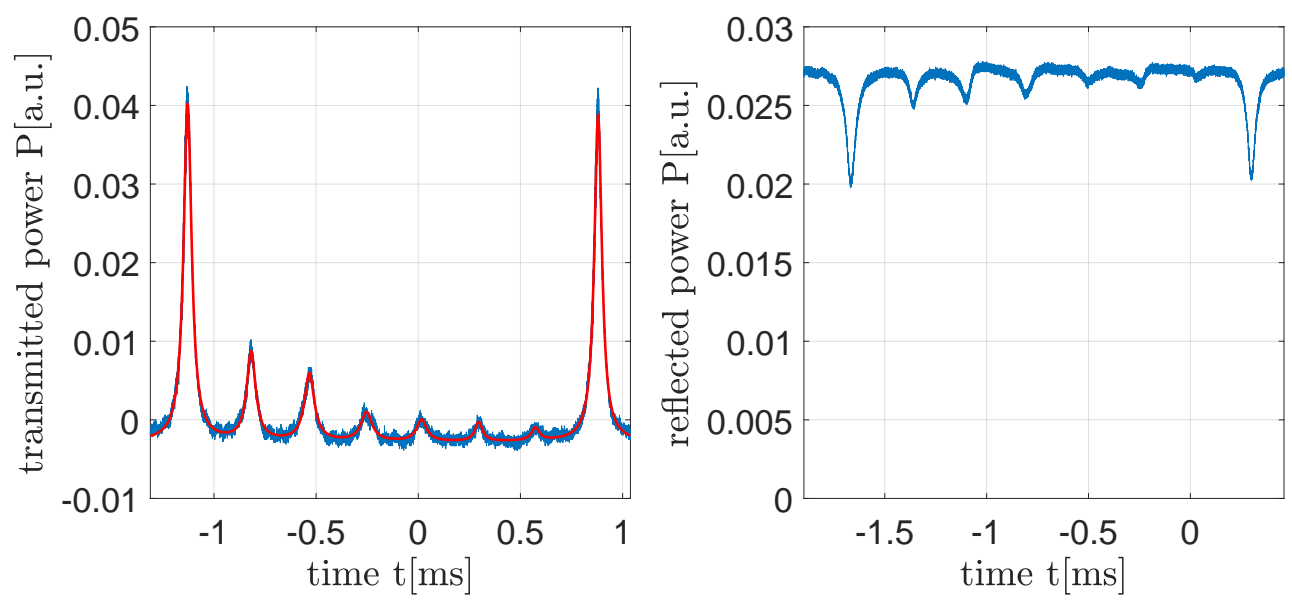

Figure A.3.: Transmitted power scan (left) together with a fit (red, sum of eight Lorentzians) and the reflected power scan (right) are shown. From the separation between modes relative to the free spectral range (FSR) the radius of curvature of the cavity mirrors can be calculated. The result is $R=500(4) \mathrm{mm}$ or $w=84(4) \mu \mathrm{m}$. From the ratio of the amplitude of the TEM00 mode to the sum of all mode amplitudes within one FSR the mode matching can be obtained. This results in 30.3\% mode matching. From the drop of the TEM00 mode a more reliable value of $28.8 \%$ for the mode matching is obtained. 
To check that the radii of curvature are equal we additionally measured the profile of the mirrors using white light interferometry (Zygo NewView 7300). Figure A.4 shows the interferogram of the output coupler $M_{2}$ (top left), the extracted surface profile (top right) and a cross section along the $x$-axis (bottom, blue) together with the fit (bottom, red). A two-dimensional sphere has been fitted eq. A.2. From the fit we extract the radius of curvature $R=497.1 \mathrm{~mm}$. With the same procedure we obtain for the input coupler $M_{1} R=494.3 \mathrm{~mm}$.

$$
z=-\sqrt{R^{2}-\left(x-x_{0}\right)^{2}-\left(y-y_{0}\right)^{2}}+z_{0}
$$
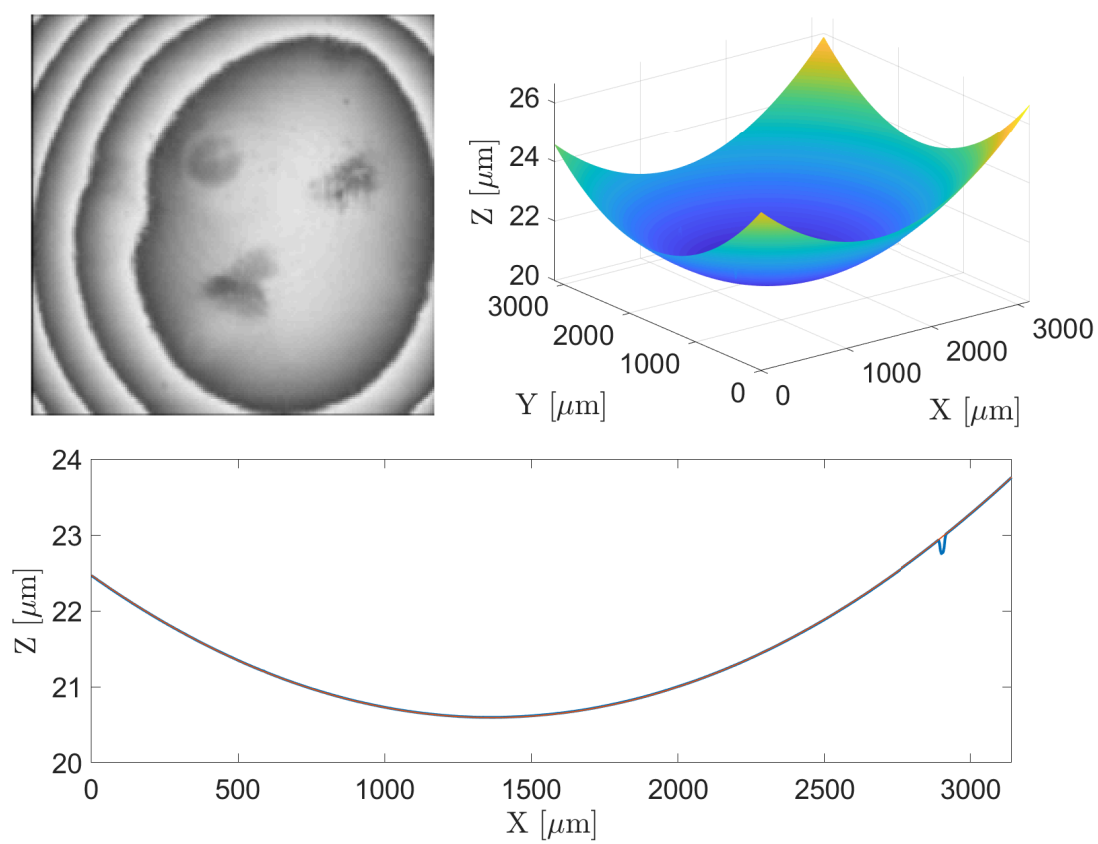

Figure A.4.: An interferogram (Zygo NewView 7300) of the output coupler $M_{2}$ (top left), the extracted surface profile (top right) and a cross section along the $x$-axis (bottom, blue) together with the fit (bottom, red) are shown. A two-dimensional sphere has been fitted using eq. A.2. From the fit we extract the radius of curvature $R=497.1 \mathrm{~mm}$. With the same procedure we obtain for the input coupler $M_{1} R=494.3 \mathrm{~mm}$. 


\section{B. AC Stark Shift Derivation}

\section{B.1. Fourth Order AC Stark Shift}

In this chapter the fourth order AC Stark shift $\left(\propto I^{2}\right)$ for CW laser and frequency comb excitations is derived. It is import to verify that the non-linearity is negligible, since the linearity assumption is explicitly used for AC Stark shift extrapolation (see seq. 5.2). As has been explained in section 2, the second order AC Stark shift $(\propto I)$ for both the CW laser and frequency comb excitations compute from the average intensity. This is a consequence of the linearity of the optical Bloch equations (see eq. 2.43) and the fact that a frequency comb can be viewed as a superposition of comb modes. However nonlinear effects, such as the fourth order AC Stark shift, are strongly enhanced if a frequency comb is used. It should be noted that odd orders of the AC Stark shift vanish. Further, due to its large spectral bandwidth, a frequency comb can be resonant with one or several of the intermediate transitions, such that additional enhancement can occur. This derivation extends the derivation in [85]. Consider the Hamiltonian $\hat{H}=\hat{H}_{0}+\hat{V}(\epsilon, t)$, where $\hat{H}_{0}$ is the free Hamiltonian of the hydrogen atom or a hydrogen-like ion and $\hat{V}(\epsilon, t)$ is the dipole interaction potential of a laser field.

$$
\hat{V}(\epsilon, t)=\hat{V} e^{-\epsilon|t|} \cos (\omega t)
$$

The laser field is assumed to be a plane, monochromatic wave polarized along the z-direction, adiabatically damped in the distance past and future. In the interaction picture $V_{I}(\epsilon, t)=e^{\frac{i}{\hbar} H_{0} t} V(\epsilon, t) e^{-\frac{i}{\hbar} H_{0} t}$, we can calculate the time-evolution operator $U_{I}(\epsilon, t)$ using the Dyson series up to the fourth order.

$$
\begin{aligned}
U_{i}(\epsilon, t)=1+\left(-\frac{i}{\hbar}\right)^{2} \int_{-\infty}^{t} d t^{\prime} \int_{-\infty}^{t^{\prime}} d t^{\prime \prime} V_{I}\left(\epsilon, t^{\prime}\right) V_{I}\left(\epsilon, t^{\prime \prime}\right)+ \\
\quad\left(-\frac{i}{\hbar}\right)^{4} \int_{-\infty}^{t} d t^{\prime} \int_{-\infty}^{t^{\prime}} d t^{\prime \prime} \int_{-\infty}^{t^{\prime \prime}} d t^{\prime \prime \prime} \int_{-\infty}^{t^{\prime \prime \prime}} d t^{\prime \prime \prime \prime} V_{I}\left(\epsilon, t^{\prime}\right) V_{I}\left(\epsilon, t^{\prime \prime}\right) V_{I}\left(\epsilon, t^{\prime \prime \prime}\right) V_{I}\left(\epsilon, t^{\prime \prime \prime \prime}\right)
\end{aligned}
$$

All odd orders of the series vanish for states with the same parity. The second order is calculated in [85] for a CW laser and can be extended for a frequency comb 
excitation. For the fourth order we obtain the following expression.

$$
\begin{aligned}
M= & \sum_{\substack{m, m^{\prime} \\
m^{\prime \prime}}} \int_{-\infty}^{t} d t^{\prime} \int_{-\infty}^{t^{\prime}} d t^{\prime \prime} \int_{-\infty}^{t^{\prime \prime}} d t^{\prime \prime \prime} \int_{-\infty}^{t^{\prime \prime \prime}} d t^{\prime \prime \prime \prime} \times \\
& \left\langle\phi\left|V_{I}\left(t^{\prime}\right)\right| m\right\rangle\left\langle m\left|V_{I}\left(t^{\prime \prime}\right)\right| m^{\prime}\right\rangle\left\langle m^{\prime}\left|V_{I}\left(t^{\prime \prime \prime}\right)\right| m^{\prime \prime}\right\rangle\left\langle m^{\prime \prime}\left|V_{I}\left(t^{\prime \prime \prime \prime}\right)\right| \phi\right\rangle \\
= & \sum_{\substack{m, m^{\prime} \\
m^{\prime \prime}, \pm}} \int_{-\infty}^{t} d t^{\prime} \int_{-\infty}^{t^{\prime}} d t^{\prime \prime} \int_{-\infty}^{t^{\prime \prime}} d t^{\prime \prime \prime} \int_{-\infty}^{t^{\prime \prime \prime}} d t^{\prime \prime \prime \prime} \\
& \times\left\langle\phi\left|e^{\frac{i}{\hbar} \hat{H}_{0} t^{\prime}-\epsilon\left|t^{\prime}\right|} \frac{\hat{V}}{2}\left(e^{i \omega_{1} t^{\prime}}+e^{-i \omega_{1} t^{\prime}}\right) e^{-\frac{i}{\hbar} \hat{H}_{0} t^{\prime}}\right| m\right\rangle \\
& \times\left\langle m\left|e^{\frac{i}{\hbar} \hat{H}_{0} t^{\prime \prime}-\epsilon \mid t^{\prime \prime}}\right| \frac{\hat{V}}{2}\left(e^{i \omega_{2} t^{\prime \prime}}+e^{-i \omega_{2} t^{\prime \prime}}\right) e^{-\frac{i}{\hbar} \hat{H}_{0} t^{\prime \prime}} \mid m^{\prime}\right\rangle \\
& \times\left\langle m^{\prime}\left|e^{\frac{i}{\hbar} \hat{H}_{0} t^{\prime \prime \prime}-\epsilon\left|t^{\prime \prime \prime}\right|} \frac{\hat{V}}{2}\left(e^{i \omega_{3} t^{\prime \prime \prime}}+e^{-i \omega_{3} t^{\prime \prime \prime}}\right) e^{-\frac{i}{\hbar} \hat{H}_{0} t^{\prime \prime \prime}}\right| m^{\prime \prime}\right\rangle \\
& \times\left\langle m^{\prime \prime}\left|e^{\frac{i}{\hbar} \hat{H}_{0} t^{\prime \prime \prime \prime}-\epsilon\left|t^{\prime \prime \prime \prime}\right|} \frac{\hat{V}}{2}\left(e^{i \omega_{4} t^{\prime \prime \prime \prime}}+e^{-i \omega_{4} t^{\prime \prime \prime \prime}}\right) e^{-\frac{i}{\hbar} \hat{H}_{0} t^{\prime \prime \prime \prime}}\right| \phi\right\rangle \\
= & \left(-\frac{i e E_{0}}{2 \hbar}\right)^{4} \frac{1}{3^{4}} \sum_{m, m^{\prime}} \int_{-\infty}^{t} d t^{\prime} \int_{-\infty}^{t^{\prime}} d t^{\prime \prime} \int_{-\infty}^{t^{\prime \prime}} d t^{\prime \prime \prime} \int_{-\infty}^{t^{\prime \prime \prime}} d t^{\prime \prime \prime \prime} \\
& \times e^{i\left(\omega_{\phi m} \pm \omega_{1}\right) t^{\prime}-\epsilon\left|t^{\prime \prime}\right|} \mu_{\phi m} e^{i\left(\omega_{m m^{\prime}} \pm \omega_{2}\right) t^{\prime \prime}-\epsilon\left|t^{\prime \prime}\right|} \mu_{m m^{\prime}} \\
& \times e^{i\left(\omega_{m^{\prime} m^{\prime \prime}} \pm \omega_{3}\right) t^{\prime \prime \prime}-\epsilon\left|t^{\prime \prime \prime}\right|} \mu_{m^{\prime} m^{\prime \prime}} e^{i\left(\omega_{m^{\prime \prime}} \phi^{ \pm \omega_{4}}\right) t^{\prime \prime \prime \prime}-\epsilon\left|t^{\prime \prime \prime \prime}\right|} \mu_{m^{\prime \prime} \phi}
\end{aligned}
$$

Where $|\phi\rangle$ is the perturbed level and the sum runs over all allowed intermediate one-photon transitions and all \pm -signs are independent, $\omega_{a b}=\omega_{a}-\omega_{b}$ and $\mu_{a b}$ are dipole matrix elements. For a CW laser $\omega=\omega_{1}=\omega_{2}=\omega_{3}=\omega_{4}$ and the indexing is introduced to better keep track of the signs. The factor $1 / 3^{4}$ takes into account, that only one polarization is considered. The integrals can be calculated successively as 
following:

$$
\begin{aligned}
& M=\left(\frac{e E_{0}}{6 \hbar}\right)^{4} \sum_{m, m^{\prime}} \int_{-\infty}^{t} d t^{\prime} \int_{-\infty}^{t^{\prime}} d t^{\prime \prime} \int_{-\infty}^{t^{\prime \prime}} d t^{\prime \prime \prime} \mu_{\phi m} \mu_{m m^{\prime}} \mu_{m^{\prime} m^{\prime \prime}} \mu_{m^{\prime \prime} \phi} \\
& m^{\prime \prime}, \pm \\
& \times e^{i\left(\omega_{\phi m} \pm \omega_{\phi}\right) t^{\prime}-\epsilon\left|t^{\prime}\right|+i\left(\omega_{m m^{\prime}} \pm \omega_{2}\right) t^{\prime \prime}-\epsilon\left|t^{\prime \prime}\right|+i\left(\omega_{m^{\prime} m^{\prime \prime}} \pm \omega_{3}\right) t^{\prime \prime \prime}-\epsilon\left|t^{\prime \prime \prime}\right|} \\
& \times \frac{e^{i\left(\omega_{m^{\prime \prime}} \pm \omega_{\phi}\right) t^{\prime \prime \prime}-\epsilon\left|t^{\prime \prime \prime}\right|}}{i\left(\omega_{m^{\prime \prime} \phi} \pm \omega_{\phi}+i \epsilon\right)} \\
& =\left(\frac{e E_{0}}{6 \hbar}\right)^{4} \sum_{\substack{m, m^{\prime} \\
m^{\prime \prime}, \pm}} \int_{-\infty}^{t} d t^{\prime} \int_{-\infty}^{t^{\prime}} d t^{\prime \prime} \mu_{\phi m} \mu_{m m^{\prime}} \mu_{m^{\prime} m^{\prime \prime}} \mu_{m^{\prime \prime} \phi} \\
& \times e^{i\left(\omega_{\phi m} \pm \omega_{1}\right) t^{\prime}-\epsilon\left|t^{\prime}\right|+i\left(\omega_{m m^{\prime}} \pm \omega_{2}\right) t^{\prime \prime}-\epsilon\left|t^{\prime \prime}\right|} \\
& \times \frac{e^{i\left(\omega_{m^{\prime} \phi} \pm \omega_{3} \pm \omega_{4}\right) t^{\prime \prime}-2 \epsilon\left|t^{\prime \prime}\right|}}{i\left(\omega_{m^{\prime \prime} \phi} \pm \omega_{4}+i \epsilon\right) i\left(\omega_{m^{\prime} \phi} \pm \omega_{4} \pm \omega_{3}+2 i \epsilon\right)} \\
& =\left(\frac{e E_{0}}{6 \hbar}\right)^{4} \sum_{\substack{m, m^{\prime} \\
m^{\prime \prime}, \pm}} \int_{\substack{t \\
m^{\prime}}}^{t} d t^{\prime} \mu_{\phi m} \mu_{m m^{\prime}} \mu_{m^{\prime} m^{\prime \prime}} \mu_{m^{\prime \prime} \phi} \\
& \times \frac{e^{i\left( \pm \omega_{1} \pm \omega_{2} \pm \omega_{3} \pm \omega_{4}\right) t^{\prime}-4 \epsilon\left|t^{\prime}\right|}}{i\left(\omega_{m^{\prime \prime} \phi} \pm \omega_{4}+i \epsilon\right) i\left(\omega_{m^{\prime} \phi} \pm \omega_{4} \pm \omega_{3}+2 i \epsilon\right) i\left(\omega_{m \phi} \pm \omega_{4} \pm \omega_{3} \pm \omega_{2}+3 i \epsilon\right)} \\
& =\left(\frac{e E_{0}}{6 \hbar}\right)^{4} \sum_{\substack{m, m^{\prime} \\
m^{\prime \prime}, \pm}} \mu_{\phi m} \mu_{m m^{\prime}} \mu_{m^{\prime} m^{\prime \prime}} \mu_{m^{\prime \prime} \phi} \\
& \times \frac{e^{i\left( \pm \omega_{1} \pm \omega_{2} \pm \omega_{3} \pm \omega_{4}\right) t^{\prime}-4 \epsilon\left|t^{\prime}\right|}}{\left(\omega_{m^{\prime \prime} \phi} \pm \omega_{4}+i \epsilon\right)\left(\omega_{m^{\prime} \phi} \pm \omega_{4} \pm \omega_{3}+2 i \epsilon\right)} \\
& \times \frac{1}{\left(\omega_{m \phi} \pm \omega_{4} \pm \omega_{3} \pm \omega_{2}+3 i \epsilon\right)\left(\omega_{4} \pm \omega_{3} \pm \omega_{2} \pm \omega_{1}+4 i \epsilon\right)}
\end{aligned}
$$

Following [85] we compute $\partial M / \partial t$, take the limit $\epsilon \rightarrow 0$ and multiply by $\hbar / i$ to obtain the fourth order AC Stark shift of a particular level $\Delta E_{A C}$. We only use terms, where $\omega_{1} \pm \omega_{2} \pm \omega_{3} \pm \omega_{4}=0$. In this case there are six possible sign combinations $\left(\omega_{1} \omega_{2} \omega_{3} \omega_{4}\right.$ $=++--,+-+1,-++-,+--+,-+-+,--++)$. If $|\phi\rangle$ is an S-state, the first intermediate state $|m\rangle$ has to be a P-state, the second $\left|m^{\prime}\right\rangle$ an S-state or D-state, the third a P-state and the final state is again $|\phi\rangle$. For $|\phi\rangle$ being the 3S-state, there is one close to resonance term in the denominator of B.4, $\omega_{\phi m^{\prime}} \pm \omega_{3} \pm \omega_{4}$, if $\left|m^{\prime}\right\rangle$ is of of the 3 D-states $\left(D_{3 / 2}\right.$ or $\left.D_{5 / 2}\right)$ and $\omega_{3}=-\omega_{4}$. The condition $\omega_{3}=-\omega_{4}$ is fulfilled 
by four out of the six possible sign combinations.

$$
E_{A C}^{(4)}=\frac{e^{4} E_{0}^{4}}{6^{4} \hbar^{4}} \sum_{\substack{m, m^{\prime} \\ m^{\prime \prime}, \pm}} \frac{\mu_{\phi m} \mu_{m m^{\prime}} \mu_{m^{\prime} m^{\prime \prime}} \mu_{m^{\prime \prime} \phi}}{\left(\omega_{m^{\prime \prime} \phi} \pm \omega_{4}\right)\left(\omega_{m^{\prime} \phi}\right)\left(\omega_{m \phi} \pm \omega_{2}\right)}
$$

Where the sums $m$ and $m^{\prime \prime}$ run over all P-states (discrete and continuum), $m^{\prime}$ over the two 3D-states and \pm over the four resonant sign combinations. With this we can write B.5 more explicitly as:

$$
E_{A C}^{(4)}=\frac{e^{4} E_{0}^{4}}{6^{4} \hbar^{4}} \sum_{d, \pm} \frac{1}{\omega_{3 d, 3 s}}\left(\sum_{n} \frac{\mu_{3 s, n p} \mu_{n p, 3 d}}{\omega_{n p, 3 s} \pm \omega_{4}}\right)\left(\sum_{n} \frac{\mu_{3 d, n p} \mu_{n p, 3 d}}{\omega_{n p, 3 s} \pm \omega_{3}}\right)
$$

Both sums in this equation are identical but their signs are independent, such that we can define for each sign term $s_{ \pm}$:

$$
s_{ \pm}=\sum_{n} \frac{\mu_{3 s, n p} \mu_{n p, 3 d}}{\omega_{n p, 3 s} \pm \omega}
$$

With this definition the fourth order AC Stark shift reduces to a simple expression.

$$
E_{A C}^{(4)}=\frac{e^{4} E_{0}^{4}}{6^{4} \hbar^{4}}\left(s_{+}+s_{-}\right)^{2} \sum_{d} \frac{1}{\omega_{3 d, 3 s}}
$$

Using atomic units with $\omega=4 / 9, \omega_{n p, 3 s}=-1 / n^{2}+1 / 9, \omega_{3 d, 3 d}=8.866 \times 10^{-7}$ for the $3 d_{3 / 2}$ and $\omega_{3 d, 3 d}=1.216 \times 10^{-6}$ for the $3 d_{5 / 2}$ transitions $(\mathrm{HFS} \ll \mathrm{FS}$ ), the sums (integrals for continuum states) of the one-photon matrix elements can be calculated analytically [31].

$$
\begin{gathered}
E_{A C}^{(4)}=\frac{e^{4} E_{0}^{4}}{6^{4} \hbar^{4}} \frac{a_{0}^{4}}{(2 \pi c R y)^{3}} \times 2.6 \times 10^{5} \\
\Delta \nu_{A C}^{(4)}=\frac{e^{4} E_{0}^{4}}{6^{4} \hbar^{4}}\left(\frac{2 I}{\epsilon_{0} c}\right)^{2} \frac{a_{0}^{4}}{(2 \pi c R y)^{3}} \times 2.6 \times 10^{5} \\
=8.6 \times 10^{-23} \frac{\mathrm{Hz}}{(\mathrm{W} / \mathrm{m})^{2}} I^{2}
\end{gathered}
$$

For comparison the second order AC Stark shift of the 1S-3S transition is:

$$
\Delta \nu_{A C}^{2}=9.8 \times 10^{-5} \frac{\mathrm{Hz}}{\mathrm{W} / \mathrm{m}} I
$$

Thus for a CW laser the second order and the fourth order AC Stark shifts are equal only at an intensity of $I=1.1 \times 10^{18} \mathrm{~W} / \mathrm{m}^{2}$. Even for the peak intensity of the frequency comb used in the current $1 \mathrm{~S}-3 \mathrm{~S}$ setup at $205 \mathrm{~nm}\left(I=4 \times 10^{9} \mathrm{~W} / \mathrm{m}^{2}\right)$, the effect is only $1.3 \mathrm{mHz}$. For the average intensity of $3 \mathrm{MW} / \mathrm{m}^{2}(80 \mu \mathrm{m}$ waist, $60 \mathrm{~mW}$ per direction) the fourth order AC Stark shift is only $77 \mathrm{nHz}$. 
Next we consider the frequency comb case. We use the same definitions of the comb in section 2, eq. 2.32. The frequency comb case differs from the $\mathrm{CW}$ case in that the 3D levels are close to the $3 \mathrm{~S}$ level, such that with several comb modes a close to resonance excitation can take place (see fig. 3.2).

$$
E(t)=\frac{E_{0}}{2} \frac{\omega_{r} \tau}{\sqrt{4 \pi}} \sum_{n} e^{-n^{2} \omega_{r}^{2} \tau^{2} / 4-i n \omega_{r} t-i \omega_{0} t}+\text { c.c }
$$

Where $E_{0}=\sqrt{2 P_{p} / \epsilon_{0} c w_{0}^{2}}$ is the peak field amplitude, $P_{p}=(2 / \pi)^{3 / 2} P_{0} T_{r} / \tau$ is the peak power, $P_{0}$ is the average power, $T_{r}=2 \pi / \omega_{r}$ is the repetition period and $\tau=$ $T_{1 / 2} / \sqrt{2 \ln (2)}\left(T_{1 / 2}\right.$ FWHM pulse duration). With this we have to modify eq. B.4 in the following way.

$$
\begin{aligned}
E_{A C}^{(4)}= & \frac{e^{4} E_{0}^{4}}{6^{4} \hbar^{4}}\left(\frac{\omega_{r} \tau}{\sqrt{2 \pi}}\right)^{4} \sum_{\substack{n_{1}, n_{2} \\
n_{3}, n_{4} \\
m^{\prime \prime}, m^{\prime} \\
m^{\prime \prime}}} \mu_{\phi m} \mu_{m m^{\prime}} \mu_{m^{\prime} m^{\prime \prime}} \mu_{m^{\prime \prime} \phi} \\
& \times \frac{e^{-\left(n_{1}^{2}+n_{2}^{2}+n_{3}^{2}+n_{4}^{2} \omega_{r}^{2} \tau^{2}\right) / 4}}{(\omega_{m^{\prime \prime} \phi} \pm \underbrace{n_{1} \omega_{r} \pm \omega_{0}}_{\approx \pm \omega_{0}})\left(\omega_{m^{\prime} \phi}-\Delta n \omega_{r}\right)(\omega_{m \phi} \pm \underbrace{n_{1} \omega_{r} \pm \omega_{0}-\Delta n \omega_{r}}_{\approx \pm \omega_{0} \text { and } \Delta n \equiv n_{2}-n_{3}})}
\end{aligned}
$$

Where we have approximated the frequencies of the individual comb modes $\left(\omega_{0}+n \omega_{r}\right)$ by the carrier frequency $\omega_{0}$, which is a good approximation if the band width of the frequency comb is not too large. For a $2 \mathrm{ps}$ comb at $205 \mathrm{~nm}$ this is very well justified, since the band width is $\approx 200 \mathrm{pm}$ only. Using $\sum_{n} e^{-n^{2} \omega_{r}^{2} \tau^{2} / 4}=\frac{\sqrt{4 \pi}}{\omega_{r} \tau}$ to sum over $n_{1}$ and $n_{4}$ we obtain:

$$
E_{A C}^{(4)}=\frac{e^{4} E_{0}^{4}}{6^{4} \hbar^{3}}\left(\frac{\omega_{r} \tau}{\sqrt{4 \pi}}\right)^{2} \sum_{\substack{n_{2}, n_{3} \\ m, m^{\prime} \\ m^{\prime \prime}, \pm}} \frac{\mu_{\phi m} \mu_{m m^{\prime}} \mu_{m^{\prime} m^{\prime \prime}} \mu_{m^{\prime \prime} \phi} e^{-\left(n_{2}^{2}+n_{3}^{2}\right) \omega_{r}^{2} \tau^{2} / 4}}{\left(\omega_{m^{\prime \prime} \phi} \pm \omega_{0}\right) \underbrace{\left(\omega_{m^{\prime} \phi}-\left(n_{2}-n_{3}\right) \omega_{r}\right)}_{\text {close to 3S-3D resonance }}\left(\omega_{m \phi} \pm \omega_{0}\right)}
$$

Again the sum $m, m^{\prime \prime}$ runs over all P-states, $m^{\prime}$ over the $3 \mathrm{D}_{3 / 2}$ and $3 \mathrm{D}_{5 / 2}$ (other terms much smaller) and \pm over the four resonant sign combinations. With this the fourth order AC Stark shift of the 3S state can be factorized in the following way.

$$
E_{A C}^{(4)}=\frac{e^{4} E_{0}^{4}}{6^{4} \hbar^{3}}\left(\frac{\omega_{r} \tau}{\sqrt{4 \pi}}\right)^{2}\left(s_{-}+s_{+}\right)^{2} \sum_{d, n_{2}, n_{3}} \frac{e^{-\left(n_{2}^{2}+n_{3}^{2}\right) \omega_{r}^{2} \tau^{2} / 4}}{\omega_{3 d, 3 s}-\left(n_{2}-n_{3}\right) \omega_{r}}
$$

The mode numbers, which lead to the smallest values in the denominator are $\omega_{3 d, 3 s}-$ $9 \omega_{r}=2 \pi \times 77.3 \mathrm{MHz}, 3 \mathrm{~S}(\mathrm{~F}=1)-3 \mathrm{D}_{3 / 2}(\mathrm{~F}=2)$ and $\omega_{3 d, 3 s}-13 \omega_{r}=2 \pi \times 98.7 \mathrm{MHz}$, $3 \mathrm{~S}(\mathrm{~F}=1)-3 \mathrm{D}_{5 / 2}(\mathrm{~F}=2)$. Thus for the $3 \mathrm{~S}$ state we obtain $\left(\omega_{r}=9.581 \times 10^{-8} \mathrm{cRy}\right.$ and $\tau=1.699 \mathrm{ps})$

$$
E_{A C}^{(4)}=\frac{e^{4} E_{0}^{4}}{6^{4} \hbar^{3}}\left(\frac{\omega_{r} \tau}{\sqrt{4 \pi}}\right)^{2} \frac{a_{0}^{4}}{(2 \pi c R y)^{3}} \times 2.9 \times 10^{9}
$$


Where $a_{0}$ is the Bohr radius. For the 1 S-3S $(F=1 \Rightarrow F=1)$ transition the fourth order AC Stark shift coefficient is:

$$
\Delta \nu_{A C}^{(4)}=\frac{e^{4} E_{0}^{4}}{6^{4} \hbar^{3}}\left(\frac{2 P_{0}}{\epsilon_{0} c w_{0}^{2}}\right)^{2}\left(\frac{2}{\pi}\right)^{2}\left(\frac{T_{r}}{\tau}\right)^{2}\left(\frac{\omega_{r} \tau}{\sqrt{4 \pi}}\right)^{2} \frac{a_{0}^{4}}{(2 \pi c R y)^{3}} \times 2.9 \times 10^{9}
$$

With this we obtain $\Delta \nu_{A C}^{(4)}=47 \mathrm{mHz} /\left(\mathrm{W} / \mathrm{m}^{2}\right)^{2} P_{0}^{2}=0.17 \mathrm{mHz}$. Comparing the fourth order AC Stark shift for $\mathrm{CW}$ and frequency comb, we obtain $\Delta \nu_{A C, C W}^{(4)}=$ $8.6 \times 10^{-23} I^{2} \mathrm{~Hz} /\left(\mathrm{W} / \mathrm{m}^{2}\right)^{2}$ and $\Delta \nu_{A C, F C}^{(4)}=1.9 \times 10^{-17}\langle I\rangle^{2} \mathrm{~Hz} /\left(\mathrm{W} / \mathrm{m}^{2}\right)^{2}$. This means that for the frequency comb case $\left(T_{1 / 2}=2 \mathrm{ps}, P_{0}=60 \mathrm{~mW}, w_{0}=80 \mu \mathrm{m}\right)$ the fourth order AC Stark shift is five orders of magnitude larger. While $\left\langle I^{2}\right\rangle /\langle I\rangle^{2} \approx 3000$, the close to resonance 3D transition additionally amplifies this effect for the frequency comb by a factor of $\approx 30$. For a frequency comb with much shorter pulses (e.g. few femtoseconds) this effect could be strongly amplified both due to a much higher peak intensity and close to resonance P-states. 



\section{Bibliography}

[1] R.H. Parker, C. Yu, W. Zhong, W. Estey, H. Müller, H. Measurement of the fine-structure constant as a test of the Standard Model. Science 360, 191-195 (2018).

[2] F. Heiße et al. High-precision measurement of the proton's atomic mass. Phys. Rev. Lett. 119, 033001 (2017).

[3] R. Pohl et al. The size of the proton. Nature 466, 213-216 (2010).

[4] P.J. Mohr, D.B. Newell, B.N. Taylor, B.N. CODATA recommended values of the fundamental physical constants: 2014. Rev. Mod. Phys. 88, 035009 (2014).

[5] A. Beyer et al. The Rydberg constant and proton size from atomic hydrogen. Science 358, 79-85 (2017).

[6] N. Bezginov, T. Valdez, M. Horbatsch, A. Marsman, A.C. Vutha, E.A. Hessels, A measurement of the atomic hydrogen Lamb shift and the proton charge radius, Science 365, 1007-1012 (2019).

[7] H. Fleurbaey et al. New Measurement of the 1S-3S transition frequency of hydrogen: contribution to the proton charge radius puzzle. Phys. Rev. Lett. 120, 183001 (2018).

[8] see for example unpublished values at the NIST website: https://physics.nist.gov/cuu/Constants/index.html

[9] A. Matveev et al. Precision measurement of the hydrogen 1S-2S frequency via a 920 km fiber link. Phys. Rev. Lett. 110, 230801 (2013).

[10] U.D. Jentschura, V.A. Yerokhin, Quantum electrodynamic corrections to the hyperfine structure of excited S states. Phys. Rev. A 73, 062503 (2006).

[11] Drake, Gordon W. F., Announcement: The Fundamental Role of Precision Measurement in Atomic, Molecular, and Optical Physics. Phys. Rev. A. 98, 1 (2018)

[12] Ångström, A. J., Recherches sur le spectre solaire : Spectre normal du soleil, atlas de six planches. (1868)

[13] Balmer, J. J., Notiz über die Spectrallinien des Wasserstoffes. Verhandlungen der Naturforschenden Gesellschaft. 7, (1885) 
[14] Michelson, A. A. and Morley, E. W., Notiz über die Spectrallinien des Wasserstoffes. Phil. Mag. 5, 24 (1887)

[15] Cohen-Tannoudji, C., Diu, B. \& Laboe F. Quantum Mechanics V2

[16] A. Antognini et al. Proton structure from the measurement of 2S-2P transition frequencies of muonic hydrogen. Science 339, 417-420 (2013).

[17] I.T. Lorenz, H.W. Hammer, U.G.Meißner, The size of the proton: Closing in on the radius puzzle. Eur. Phys. J. A 48, 151 (2012).

[18] G. Hagel, F. Nez, F. Biraben, Analysis and observation, on an atomic resonance, of the frequency shift due to the length modulation of an optical cavity. Appl. Opt. 41, 7702-7706 (2002).

[19] M. Herrmann et al. Feasibility of coherent XUV spectroscopy on the 1S-2S transition in singly ionized helium. Phys. Rev. A 79, 052505 (2009).

[20] Arnoult, O., Nez, F., Julien, L. \& Biraben, F. Optical frequency measurement of the 1S-3S two-photon transition in hydrogen. Eur. Phys. J. D 60, 2 (2010)

[21] D.C. Yost et al. Spectroscopy of the hydrogen 1S-3S transition with chirped laser pulses. Phys. Rev. A 93, 042509 (2016).

[22] Th. Udem, R. Holzwarth, T.W. Hänsch, Optical Frequency Metrology. Nature 416, 233-237 (2004).

[23] Adler, F., Thorpe, M. J., Cossel, K. C. \& Ye, J. Cavity-Enhanced Direct Frequency Comb Spectroscopy: Technology and Applications. Annu. Rev. Anal. Chem. 3, 1 (2010)

[24] M.C. Stowe et al. Direct frequency comb spectroscopy. Adv. Atom. Mol. Opt. Phy. 55, 1-60 (2008).

[25] Ye.V. Baklanov, V.P. Chebotayev, Narrow resonances of two-photon absorption of super-narrow pulses in a gas. Appl. Phys. 12, 97-99 (1977).

[26] S. Reinhardt, E. Peters, T.W. Hänsch, Th. Udem, Two-photon direct frequency comb spectroscopy with chirped pulses. Phys. Rev. A 81, 033427 (2010).

[27] Teets, R., Eckstein, J. \& Hänsch, T. W.. Coherent two-photon excitation by multiple light pulses. Phys. Rev. Lett. 38, 760-764 (1977)

[28] J.N. Eckstein, A.I. Ferguson, T.W. Hänsch, High-resolution two-photon spectroscopy with picosecond light pulses. Phys. Rev, Lett. 40, 847-850 (1978).

[29] Thomas Udem, Habilitationsschrift, "Die Messung der Frequenz von Licht mit modengekoppelten Lasern. (2002) 
[30] M. Haas, U.D. Jentschura, C.M. Keitel, N. Kolachevsky, M. Herrmann, P. Fendel, M. Fischer, Th. Udem, R. Holzwarth, T.W. Hänsch, Two-photon excitation dynamics in bound two-body Coulomb systems including AC Stark shift and ionization. Phys. Rev. A 73, 052501 (2006).

[31] Stobbe, M. Zur Quantenmechanik photoelektrischer Prozesse. Ann. Phys. 399, 661-715 (1930)

[32] Edmonds, A. R., Angular momentum in quantum mechanics (1996)

[33] Bonin, K. D., \& McIlrath, T. J. Two-photon electric-dipole selection rules. J. Opt. Soc. Am. B 1, 52 (1984)

[34] Peters, E. PhD Thesis. Experimental and theoretical investigation of direct frequency comb spectroscopy (2011)

[35] Matveev A. et al., To be published.

[36] H. Pauly, G. Scoles, (Ed.) Atomic and Molecular Beam Methods. (Oxford University Press, New York, Oxford 1988).

[37] Th. Udem, Quantum electrodynamics and the proton size. Nat. Phys. 14, 632 (2018).

[38] W. Xiong et al. A small proton charge radius from an electron-proton scattering experiment. Nature 575, 147-151 (2019).

[39] R.W.P. Drever, J.L. Hall, F.V. Kowalski, J. Hough, G.M. Ford, A.J. Munley, Laser phase and frequency stabilization using an optical resonator. Appl. Phys. B 31, 97-105 (1983).

[40] E. Peters, D.C. Yost, A. Matveev, T.W. Hänsch, Th. Udem, Frequency comb spectroscopy of the hydrogen 1S-3S and 1S-3D transitions. Ann. d. Phys. 525, L29-L34 (2013).

[41] E. Peters, S.A. Diddams, P. Fendel, S. Reinhardt, T.W. Hänsch, Th. Udem, A deep-UV optical frequency comb at 205 nm. Opt. Expr. 17, 9183-9190 (2009).

[42] G. Grynberg, F. Biraben, E. Giacobino, B. Cagnac, Doppler-free two-Photon Spectroscopy of Neon. II. Line Intensities. J. de Phys. 38, 629-640 (1977).

[43] P. Fendel, S.D. Bergeson, Th. Udem, T.W. Hänsch, Two-photon frequency comb spectroscopy of the 6s-8s transition in cesium. Opt. Lett. 32, 701-703 (2007).

[44] A. Ozawa, Y. Kobayashi, Chirped-pulse direct frequency-comb spectroscopy of two-photon transitions. Phys. Rev. A 86, 022514 (2012). 
[45] H. Wang, A.M. Weiner, Efficiency of short-pulse type-I second-harmonic generation with simultaneous spatial walk-off, temporal walk-off, and pump depletion. IEEE J. Quant. Electr. 39, 1600-1618 (2003).

[46] G. Hagel, R. Battesti, F. Nez, L. Julien, F. Biraben, Observation of a motional Stark effect to determine the second-order Doppler effect. Phys. Rev. Lett. 89, 203001 (2002).

[47] D.C. Yost, A. Matveev, E. Peters, A. Beyer, T.W. Hänsch, Th. Udem, Quantum interference in two-photon frequency-comb spectroscopy. Phys. Rev. A 90, 012512 (2014).

[48] H. Fleurbaey, F. Biraben, L. Julien, J.P. Karr, F. Nez, Cross-damping effects in 1S-3S spectroscopy of hydrogen and deuterium. Phys. Rev. A 95, 052503 (2017).

[49] V.A. Yerokhin, K. Pachucki, V. Patkóš Theory of the Lamb shift in hydrogen and light hydrogen-like ions. Ann. Phys. 531, 1800324 (2019).

[50] http://doi.org/10.5281/zenodo.4046497.

[51] S.T. Dawkins, J.J. McFerran, A.N. Luiten, Considerations on the measurement of the stability of oscillators with frequency counters. IEEE Trans. Ultr. Fer. Freq. Contr. 54, 918-925 (2007).

[52] T.W. Hänsch, B. Couillaud, Laser frequency stabilization by polarization spectroscopy of a reflecting reference cavity. Opt. Commun. 35, 441 (1980).

[53] J. Hollenshead, L. Klebanoff, Modeling radiation-induced carbon contamination of extreme ultraviolet optics. J. Vac. Sci. Technol. B 24, 64-82 (2006).

[54] J.T.M. Walraven, I.F. Sivera, Helium-temperature beam source of atomic hydrogen. Rev. Sci. Instrum. 53, 1167-1181 (1982).

[55] D. Singy, P.A. Schmelzbach, W. Grübler, Z.W. Zhang, Study of the surface recombination in the production of a dense polarized hydrogen atomic beam at low temperature. Nuc. Instr. and Meth. in Phys. Res. B 47, 167-180 (1990).

[56] G. Hagel, R. Battesti, F. Nez, L. Julien, F. Biraben, Observation of a motional Stark effect to determine the second-order Doppler effect. Phys. Rev. Lett. 89, 203001 (2002).

[57] H.A. Bethe, E.F. Salpeter, Quantum Mechannics of one- and two-electron atoms. (Springer, Berlin, Göttingen, Heidelberg 1957).

[58] N.S. Andreev, A.V. Emeline, S.V. Polikhova, V.K. Ryabchuk, N. Serpone, Photoinduced adsorption of hydrogen and methane on $\gamma$-alumina. The photoinduced chesorluminescence (PhICL) effect, Langmuir 20, 129-135 (2004). 
[59] J. Baker, Transition probabilities for one electron atoms. Natl. Inst. Stand. Technol. Technical Note 1612, 1-8 (2008).

[60] private communication, Savely Karshenboim.

[61] N.F. Ramsey, Molecular Beams. (Oxford University Press Oxford 1990).

[62] S.F. Cooper, D.A. Brandt, C. Rasor, Z. Burkley, D.C. Yost, Cryogenic atomic hydrogen beam apparatus with velocity characterization, Rev. Sci. Instrum. 91, 013201 (2020).

[63] Alexey Grinin, "Two-Photon Frequency Comb Spectroscopy of atomic Hydrogen", thesis Ludwig-Maximilians-Universität München, 2020.

[64] Christian Parthey, "Precision spectroscopy on atomic hydrogen", thesis LudwigMaximilians-Universität München, 2011.

[65] J.W. Farley, W.H. Wing, Accurate calculation of dynamic Stark shifts and depopulation rates of Rydberg energy levels induced by blackbody radiation. Hydrogen, helium, and alkali-metal atoms. Phys. Rev. A 23, 2397 (1981).

[66] J. Vanier, C. Audoin The quantum physics of atomic frequency standards. Vol. I, IOP publishing (1989).

[67] I.I. Sobelman, L.A. Vainshtein, E.A. Yukov, Excitation of Atoms and Broadening of Spectral Lines. (Springer, Berlin 1981).

[68] C.M. Adhikari, V. Debierre, U.D. Jentschura, Long-range interactions of hydrogen atoms in excited states. III. nS-1S interactions for $n \geq 3$. Phys. Rev. A, 96, 032702 (2017).

[69] A. Matveev, N. Kolachevsky, C.M. Adhikari, U.D. Jentschura, Pressure shifts in high-precision hydrogen spectroscopy: II. Impact approximation and MonteCarlo simulations. J. Phys. B 52, 075006 (2019).

[70] Th. Udem, L. Maisenbacher, A. Matveev, V. Andreev, A. Grinin, A. Beyer, N. Kolachevsky, R. Pohl, D.C. Yost, T.W. Hänsch, Quantum interference line shifts of broad dipole-allowed transitions. Ann. Phys. 531, 1900044 (2019).

[71] R.C. Brown, A. Wu, J.V. Porto, C.J. Sansonetti, C.E. Simien, S.M. Brewer, J.N. Tan, J.D. Gillaspy, Quantum interference and light polarization effects in unresolvable atomic lines: Application to a precise measurement of the ${ }^{6,7} \mathrm{Li} \mathrm{D}_{2}$ lines. Phys. Rev. A 87, 032504 (2013).

[72] M. Horbatsch, E.A. Hessels, Shifts from a distant neighboring resonance. Phys. Rev. A 82, 052519 (2010). 
[73] A.A. Buchheit, G. Morigi, Master equation for high-precision spectroscopy. Phys. Rev. A 94, 042111 (2016).

[74] M. Abramowitz, I. Stegun, Handbook of Mathematical Functions. (Dover Books on Mathematics, 1965).

[75] G.P. Agrawal, Nonlinear fiber Optics. (Academic Press, San Diego 2001).

[76] J.Y. Zhang, J.Y. Huang, H. Wang, K.S. Wong, G.K. Wong, Second-harmonic generation from regeneratively amplified femtosecond laser pulses in BBO and LBO crystals. J. Opt. Soc. Am. B 15, 200 (1998).

[77] I.V. Hertel, C.P. Schulz, Atoms, molecules and optical physics. (Springer, Berlin 2015).

[78] L. Essen, R.W. Donaldson, M.J. Bangham, E.G. Hope, Frequency of the hydrogen maser. Nature 229, 110 (1971).

[79] D.Z. Anderson, Alignment of resonant optical cavities. Appl. Opt. 23, 2944-2949 (1984).

[80] Snadden, M. J., Bell, A. S., Riis, E. \& Ferguson, A. I. Two-photon spectroscopy of laser-cooled Rb using a mode-locked laser. Opt. Commun. 125, 70-76 (1996)

[81] Marian, A., Stowe, M. C., Felinto, D. \& Ye, J. Direct frequency comb measurements of absolute optical frequencies and population transfer dynamics. Phys. Rev. Lett. 95, 023001 (2005)

[82] Gerginov, V., Tanner, C. E., Diddams, S., Bartels, A. \& Hollberg, L. High resolution spectroscopy with a femtosecond laser frequency comb. 2005 Conf. Lasers Electro-Optics CLEO. 2, 1249-1251 (2005)

[83] Fortier, T. M., et al.. Kilohertz-resolution spectroscopy of cold atoms with an optical frequency comb. Phys. Rev. Lett. 97, 163905 (2006)

[84] Crampton S.B. Hydrogen atom adsorption on molecular solid surfaces, Ann. Phys. Fr., 10, 893-900 (1985).

[85] Haas, M., Jentschura, U. D., \& Keitel, C. H. Comparison of classical and second quantized description of the dynamic Stark shift. Am. J. Phys. 74, 77-81 (2006) 


\section{Acknowledgments}

I would like to thank Professor Theodor Hänsch for giving me the opportunity to work in his group during my Master thesis and later PhD thesis during the last seven years. These years were an incredible possibility to learn, to get experience, present results at conferences around the world and most importantly to contribute to real scientific progress, which is done in the laser spectroscopy department at the MPQ. Thank you very much!

I would like to thank Professor Thomas Udem, who was supervising my PhD Thesis (and also master thesis). Thomas, there are only very seldom such group leaders like you, which know so much about the experiments, actively work on the current problems and results and contribute a large portion of the work, while supervising several different experiments at the same time! Thank you very much for your help and your large contribution to the results, which have been achieved. Although we have not been speaking always the same scientific language and not always took the same approach, I think, that your precise, detailed and accurate style, will strongly influence my future scientific work. Thank you very much! I enjoyed our discussions both scientific and nonscientific very much and I learned very much from you both scientifically and personally.

Dear Arthur! Thank you very much for these years, which we worked together! It was a great time for me! I don't need to mention, that your contribution to the experiment is immense! I want to thank you for the support in lab and simulations, for the countless fruitful discussions, we had, on how to make possible and to improve the measurement! I learned so much from you! I wish you all the best in your future career and personal life!

I would like to thank Dylan Yost for introducing me into the experiment during the first year! This year was very exciting and you are a very talented supervisor! Thanks for making so many things in the 1S-3S lab work, such that this great result could be achieved within my PhD thesis! Thanks a lot, that even after you left, you still helped me over skype and email and for your many visits to MPQ during which you helped with the experiment! I think even being thousands of kilometers away, you stayed an active member of the 1S-3S experiment during all these years!

I would like to thank my hydrogen group members: Lothar Maisenbacher, Randolf Pohl and Vitaly Wirthl. Thank you so much for the countless meetings and personal discussions, which we had, for the helpful advices and tips you gave me, for your support and direct contributions to the experiment. The 1S-3S experiment is without doubt a team work! I wish you all the best in your career and personal life!

I also would like to thank Akira Ozawa, Johannes Weitenberg and Fabian Schmid for your help on many experimental and theoretical issues during these years! A 
special thank to Vladimir Pervak, Alexander Guggenmos and Yang Cui for your the laser mirror characterization measurements you performed for me!

I would like to thank our technicians Wolfgang Simon and Karl Linner for the incredible help with all parts you made for me during the course of this work. You did not only machine countless part but also helped designing and mounting them! I also would like to thank Helmut Brückner for his help with all our electronics! I also would like to thank Ingrid Hermann for her help! I really appreciate your work and support of the scientific work!

I would like to thank my parents for their support! All my achievements are the fruits of your wise parenting and care from my birth on!

Finally and most importantly, I would like to thank my wife - Yuliya! You are the best wife, one could imagine! During all these years you took most of the work at home and with our kids, letting me concentrate on my $\mathrm{PhD}$ project, even on Sundays. Your love and care were the strongest hold and motivation for me. Your contribution to this work is the largest! 


\section{List of publications and presentations}

\section{List of publications}

1. A. Grinin, A. Matveev, D. C. Yost, L. Maisenbacher, V. Wirthl, R. Pohl, T.W. Hänsch and Th. Udem. Two-Photon Frequency Comb Spectroscopy of Atomic Hydrogen. Science, accepted on 15.10.2020.

2. A. Beyer, L. Maisenbacher, A. Matveev, R. Pohl, A. Grinin, K. Khabarova, Y. Chang, T. Lamour, T. Shi, D.C. Yost, Th. Udem, T. W. Hänsch, N. Kolachevsky: An active fiber-based retroreflector providing phase-retracing antiparallel laser beams for precision spectroscopy. Optics Express 24, 017470, (2016)

3. D. C. Yost, A. Matveev, A. Grinin, E. Peters, L. Maisenbacher, A. Beyer, R. Pohl, N. Kolachevsky, K. Khabarova, T. W. Hänsch, and Th. Udem. Spectroscopy of the hydrogen 1S-3S transition with chirped laser pulses. Phys. Rev. A 93, 042509, (2016)

4. A. Beyer, L. Maisenbacher, A. Matveev, R. Pohl, K. Khabarova, A. Grinin, T. Lamour, D. C. Yost, T. W. Hänsch, N. Kolachevsky, Th. Udem: The Rydberg constant and proton size from atomic hydrogen. Science, Vol. 358, Issue $6359,(2017)$

5. R. Pohl, F. Nez, Th. Udem, A. Antognini, A. Beyer, H. Fleurbaey, A. Grinin, T. W. Hänsch, L. Julien, F. Kottmann. Deuteron charge radius and Rydberg constant from spectroscopy data in atomic deuterium. Metrologia, Vol.54, Number 2, (2017)

6. Th. Udem, L. Maisenbacher, A. Matveev, V. Andreev, A. Grinin, A. Beyer, N. Kolachevsky, R. Pohl, D. C. Yost, T. W. Hänsch. Quantum Interference Line Shifts of Broad Dipole-Allowed Transitions. Ann. Phys. (Berlin) 531, 1900044, (2019) 


\section{Invited talks}

Budabest, Hungary (2015) Conference on Precision Physics and Fundamental Constants

Fort Collins, USA (2017) Physics, Colloquim Talk,

Colorado State University (CSU)

Los Angeles, USA (2018) AMO Colloquim Seminar at UCLA

University of California, Los Angeles

Vienna, Austria (2018)

The International Conference on Precision Physics of Simple Atomic Systems (PSAS2018)

Toronto, Canada (2019) Physics Colloquim

University of Toronto

Toronto, Canada (2019) Physics Colloquim

York Univerity,

Garching, DE (2019) Colloquium at the

Max Planck Institute of Quantum Optics 


\section{Contributed Presentations}

Castle Ringberg, DE (2014 - 2010)

Ein Gedi, Israel (2014)
Annual Symposium of the Laser Spectroscopy Division at the MPI of Quantum Optics (one talk per year)

French-Israel Symposium on non-linear and Quantum Optics 



\section{Declaration of Originality}

I hereby declare that this dissertation represents my original work and that I did not use sources and auxiliary means other than those given in the text.

Munich, June $17^{\text {rd }}, 2020$.

Alexey Grinin 
